Florida International University FIU Digital Commons

\title{
Evaluation of the Scent Collection System for Its Effectiveness in Volatile Organic Compound Collection and Use in Canine Training
}

\author{
Claudia L. Sanchez Ms \\ Florida International University, csanc035@fiu.edu
}

DOI: $10.25148 /$ etd.FI15032116

Follow this and additional works at: https://digitalcommons.fiu.edu/etd

Part of the Analytical Chemistry Commons

\section{Recommended Citation}

Sanchez, Claudia L. Ms, "Evaluation of the Scent Collection System for Its Effectiveness in Volatile Organic Compound Collection and Use in Canine Training" (2015). FIU Electronic Theses and Dissertations. 1914.

https://digitalcommons.fiu.edu/etd/1914 


\title{
FLORIDA INTERNATIONAL UNIVERSITY
}

Miami, Florida

EVALUATION OF THE SCENT COLLECTION SYSTEM FOR ITS

\section{EFFECTIVENESS IN VOLATILE ORGANIC COMPOUND COLLECTION AND}

USE IN CANINE TRAINING

\author{
A thesis submitted in partial fulfillment of \\ the requirements for the degree of \\ MASTER OF SCIENCE \\ in \\ FORENSIC SCIENCE \\ by
}

Claudia Liliana Sánchez 
To: Dean Michael R. Heithaus

College of Arts and Sciences

This thesis, written by Claudia Liliana Sánchez, and entitled Evaluation of the Scent Collection System for its Effectiveness in Volatile Organic Compound Collection and Use in Canine Training, having been approved in respect to style and intellectual content, is referred to you for judgment.

We have read this thesis and recommend that it be approved.

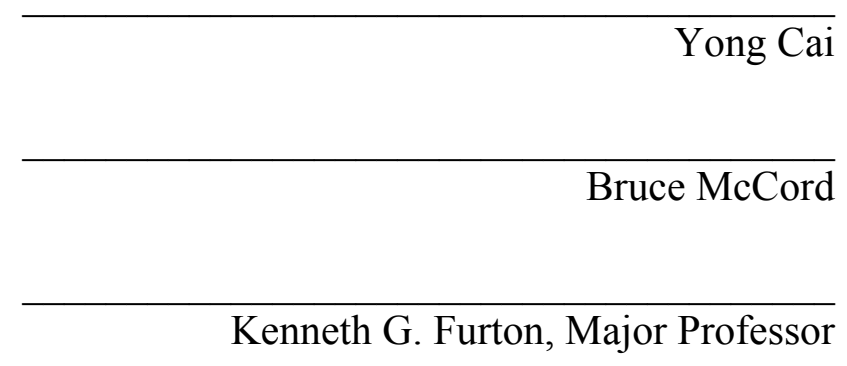

Date of Defense: March 16, 2015

The Thesis of Claudia Liliana Sánchez is approved.

\begin{tabular}{r}
\hline $\begin{array}{c}\text { Dean Michael R. Heithaus } \\
\text { College of Arts and Sciences }\end{array}$ \\
\hline Dean Lakshmi N. Reddi \\
University Graduate School
\end{tabular}

Florida International University, 2015 
(C) Copyright 2015 by Claudia Liliana Sánchez

All rights reserved. 


\section{DEDICATION}

This thesis work is dedicated to my husband, Gerardo, for his love, support, and encouragement. I am truly thankful for having you in my life. This work is also dedicated to my mother, Rosa Maria, who has been a source of inspiration through my life. And also thanks to all members of my family who have always loved me unconditionally and whose good examples have taught me to work hard for the things that I aspire to achieve. 


\section{ACKNOWLEDGMENTS}

First and foremost, I want to thank my advisor, Dr. Kenneth G. Furton for his guidance and especially for his academic support as well as his confidence in me. I would also like to thank my committee members, Dr. Bruce McCord and Dr. Yong Cai who provided encouraging and constructive feedback. I want to express my gratitude to Adhly M. Huertas, my fellow partner in this project for her friendship, effort, caring, and patience along this journey. In addition, I'd like to thank Dr. Paola Prada and Dr. Howard Holness for their invaluably constructive criticism and friendly advice during this project.

I also would like to show my deepest appreciation to my cousin Alexandra Villescas for her devotion, unconditional love, support, sense of humor, patience, optimism, and moral support especially during difficult times. I am sincerely grateful to all members of Dr. Furton's research group who supported me throughout the course of this research project sharing their truthful and illuminating views on a number of issues.

I express my warm thanks to City of Miami Police Department, Metro Dade K9 facility, and United States Marine Corps (California) for granting me access to their canine teams and explosives. Special thanks to Peter Núñez and Bob Suarez who provided me with some material being required for this project.

I want to give a special gratitude to Jessie Guevara for her diligence and assistance in this project. I also gratefully acknowledge the Technical Support Working Group (TSWG) for the financial support that I have received while working on this project.

Thank you Lord for the wisdom, perseverance, and for always being there for me...! 


\author{
ABSTRACT OF THE THESIS \\ EVALUATION OF THE SCENT COLLECTION SYSTEM FOR ITS \\ EFFECTIVENESS IN VOLATILE ORGANIC COMPOUND COLLECTION AND \\ USE IN CANINE TRAINING \\ by
}

Claudia Liliana Sánchez

Florida International University, 2015

Miami, Florida

Professor Kenneth G. Furton, Major Professor

As a result of increased terrorist activity around the world, the development of a canine training aid suitable for daily military operations is necessary to provide effective canine explosive detection. Since the use of sniffer dogs has proven to be a reliable resource for the rapid detection of explosive volatiles organic compounds, the present study evaluated the ability of the Human Scent Collection System (HSCS) device for the creation of training aids for plasticized / tagged explosives, nitroglycerin and TNT containing explosives, and smokeless powders for canine training purposes. Through canine field testing, it was demonstrated that volatiles dynamically collected from real explosive material provided a positive canine response showing the effectiveness of the HSCS in creating canine training aids that can be used immediately or up to several weeks (3) after collection under proper storage conditions. These reliable non-hazardous training aids allow its use in areas where real explosive material aids are not practical and/or available. 


\section{TABLE OF CONTENTS}

CHAPTER

PAGE

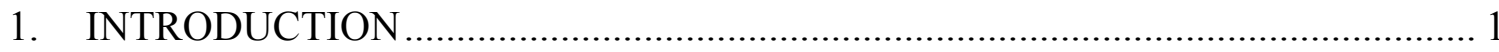

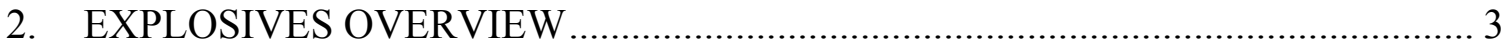

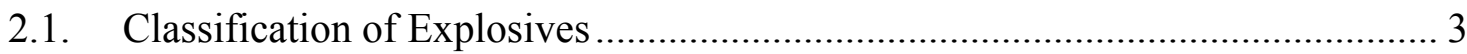

2.1.1. Low and High Explosives..................................................................... 3

2.1.2. Chemical Components of Explosives ........................................................ 5

2.1.3. Other Constituents of Explosives......................................................... 7

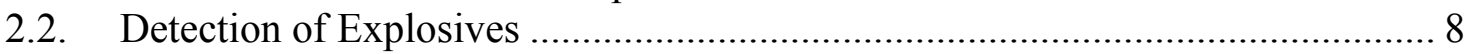

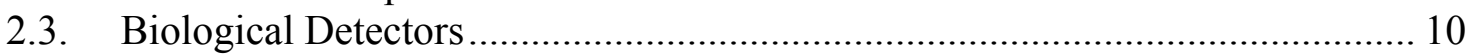

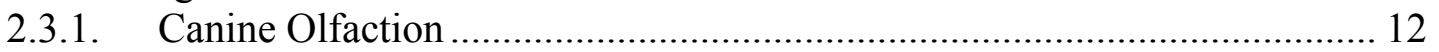

2.3.2. Canine Detection and Explosive VOCs ………........................................ 14

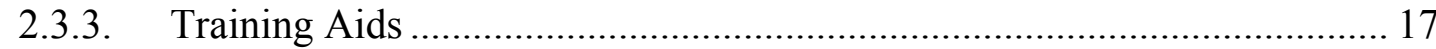

2.3.4. Canine Detectors and the Law ……………......................................... 20

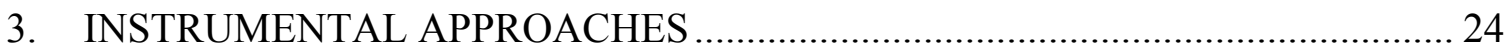

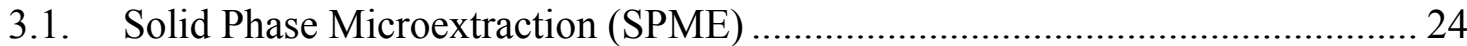

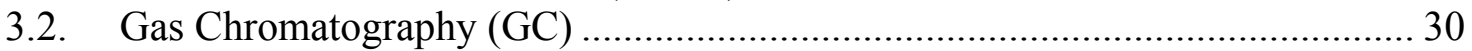

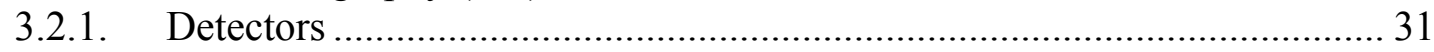

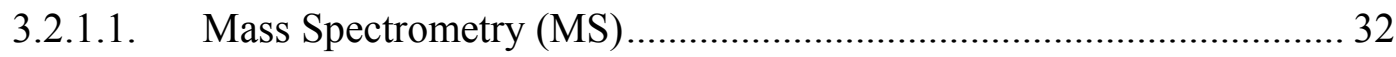

3.2.1.2. Electron Capture Detector (ECD) ..................................................... 32

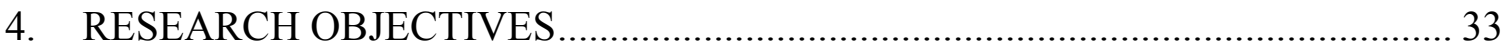

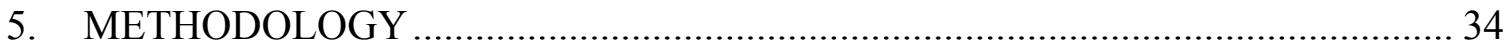

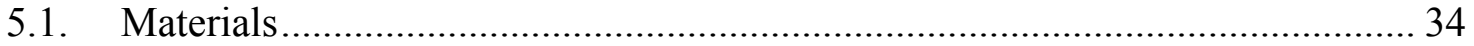

5.1.1. Explosive materials ............................................................................ 34

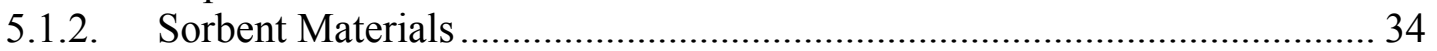

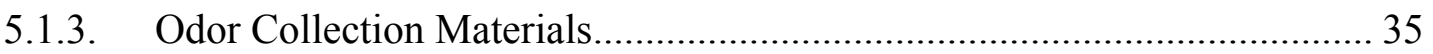

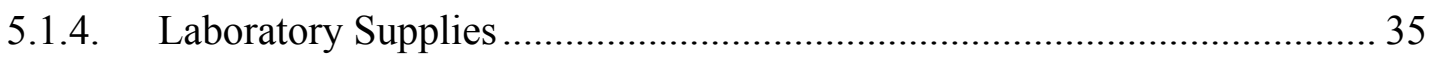

5.2. Statistical Data Analysis............................................................................... 36

5.3. HSCS Device for Collection of Explosive Volatiles ......................................... 38

5.3.1. HSCS Airflow Velocity ........................................................................ 40

5.3.2. HSCS Protocol for Collection of Explosive Volatiles.................................. 43

5.4. GC/MS Method for Analysis of 2E1H and DMNB Representative VOCs ....... 44

5.5. GC/ECD Method for Analysis of 2, 4 DNT and NG Representative VOCs ..... 45

5.6. SPME Analysis and Extraction Procedures ......................................................... 46

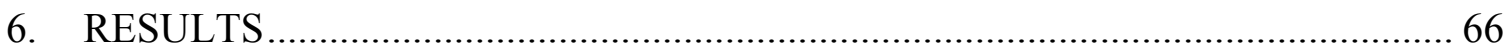

6.1. TASK 1: EVALUATION OF EXPLOSIVE FAMILIES ................................... 66

6.1.1. SPME Extraction and Chromatographic Analysis of Extracted VOCs ...... 68 
6.1.1.1. 2-ethyl-1-hexanol (2E1H) and 2, 3-Dimethyl-dinitrobutane (DMNB) Representative Volatiles from $\mathrm{C} 4$.

6.1.1.2. 2,4- Dinitrotoluene (2,4 -DNT) Representative Volatile from Single Based Smokeless Powder and TNT 70

6.1.1.3. Nitroglycerin (NG) Representative Volatile from Double Based Smokeless Powder 72

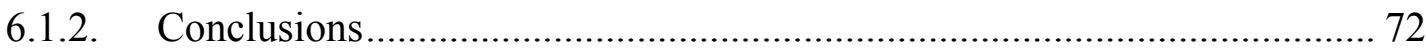

\subsection{TASK 2: EVALUATION AND OPTIMIZATION OF FLOW RATE OF THE} HSCS USING REPRESENTATIVE VOCS....................................................... 73

6.2.1. 2-ethyl-1-hexanol (2E1H) and 2, 3-Dimethyl-dinitrobutane (DMNB) ...... 74

6.2.1.1. SPME Extraction Time Optimization................................................ 74

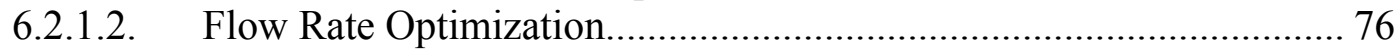

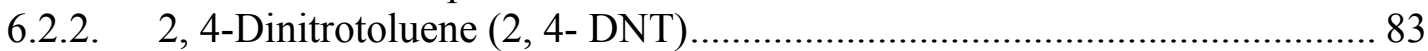

6.2.2.1. SPME Extraction Time Optimization................................................. 83

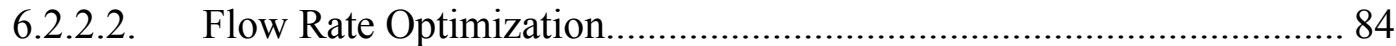

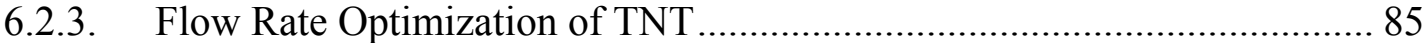

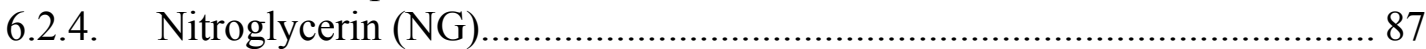

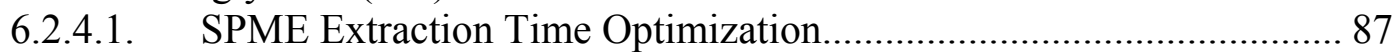

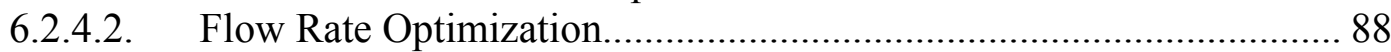

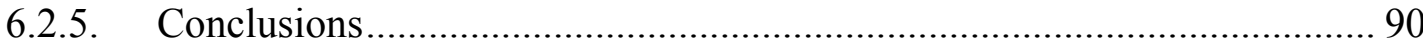

6.3. TASK 3: EVALUATION AND OPTIMIZATION OF SAMPLING TIME (30 SEC V. 60 SEC) OF REPRESENTATIVE VOCS ACCUMULATION USING THE

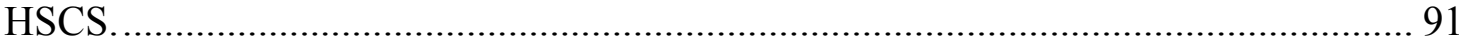

6.3.1. Sampling Time of 2E1H and DMNB Representative Volatiles ................. 91

6.3.2. HSCS Collection of C4 Explosive Material at Optimal Parameters........... 93

6.3.3. Sampling Time of 2, 4-DNT Representative Volatile ................................. 99

6.3.4. Sampling Time of 2,4 DNT Representative Volatile from TNT ............... 102

6.3.5. Sampling Time of Nitroglycerin Representative Volatile ......................... 105

6.3.6. Comparison of HSCS Collection of 2,4-DNT Volatile from TNT and Single Based Smokeless Powder........................................................................ 107

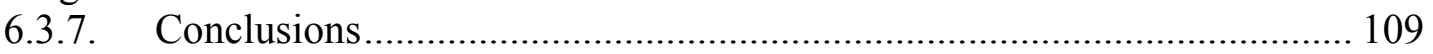

6.4. TASK 4: COMPARISON OF STATIC VS. DYNAMIC COLLECTION....... 111 6.4.1. Static Collection of 2E1H and DMNB Representative VOCs from C4 ... 112 6.4.2. Comparison of Static vs. Dynamic Collection of 2E1H and DMNB

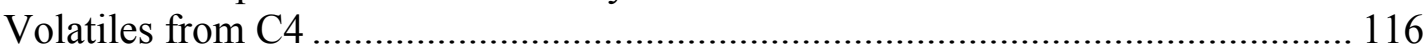
6.4.3. Static Collection of 2, 4-DNT Volatile from Single Based Smokeless

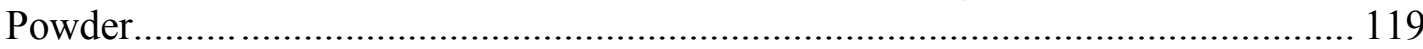
6.4.4. Comparison of Dynamic vs. Static Collection of 2,4-DNT Volatile from Single Based Smokeless Powder. 122

6.4.5. Static Collection of Nitroglycerin Volatile from Double Based Smokeless Powder. 
6.4.6. Comparison of Dynamic vs. Static Collection of Nitroglycerin Volatile from Double Based Smokeless Powder ............................................................ 125

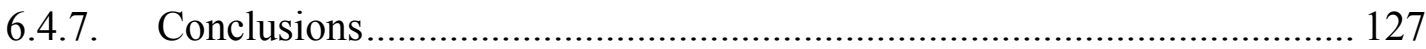

6.5. EVALUATION OF LONGEVITY OF REPRESENTATIVE VOCS AND SELECTION OF AN OPTIMAL STORAGE CONTAINMENT SYSTEM .............. 128

6.5.1. Persistence of Target Volatiles 2E1H and DMNB and Optimal Storage

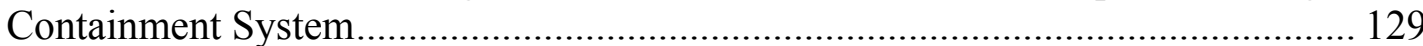

6.5.2. Persistence of Target Volatiles 2,4-DNT and NG and Optimal Storage

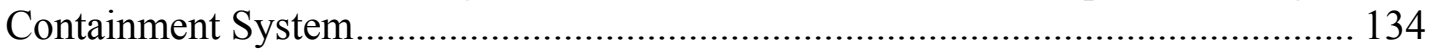

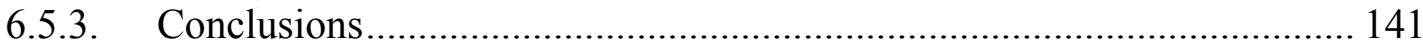

6.6. TASK 6: FIELD EVALUATIONS OF THE HSCS USING EXPLOSIVE

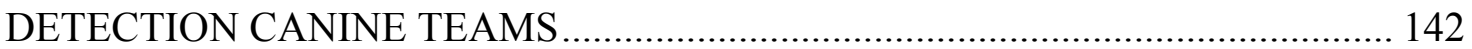

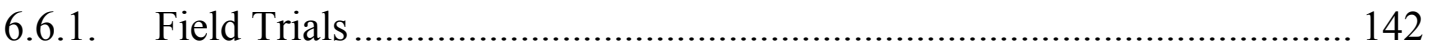

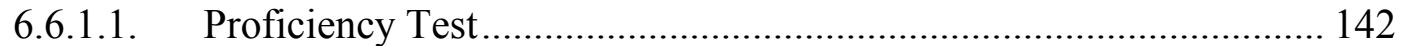

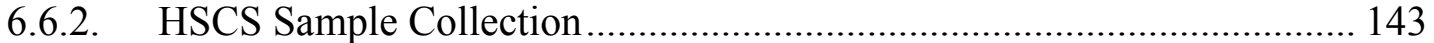

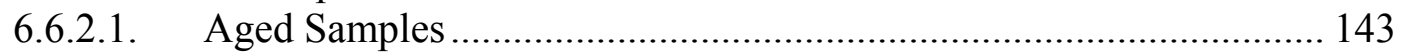

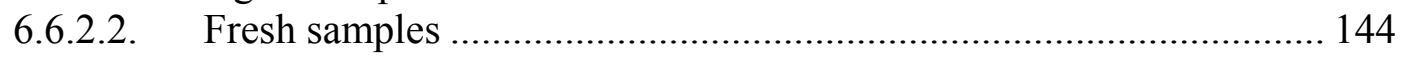

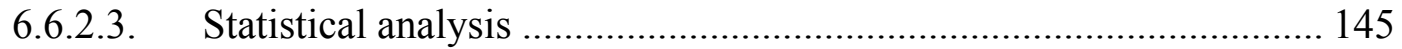

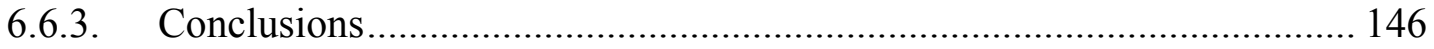

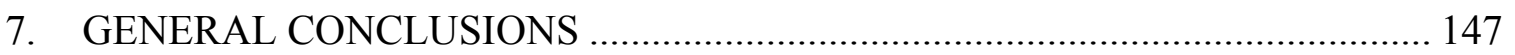

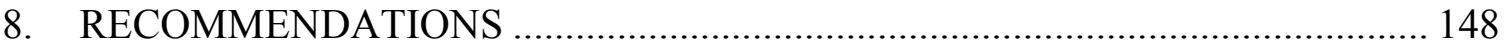

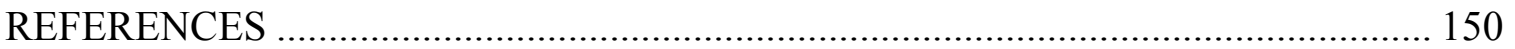

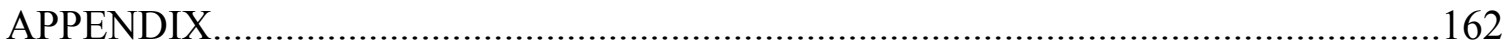




\section{LIST OF TABLES}

TABLE

PAGE

Table 1: Summary of Fiber Coatings Available for SPME Fibers ............................... 28

Table 2 Calculations for the HSCS Air Flow Velocity Measurements ......................... 41

Table 3. Summary of the Air Flow Volume Measurements ......................................... 43

Table 4: Evaluated Parameters for Selected Explosive Material.................................... 64

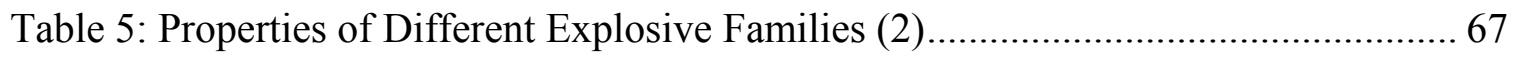

Table 6: 2E1H Mass Average Collected form C4 Explosive at all Flow Rates at

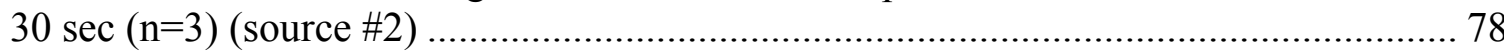

Table 7: DMNB Mass Average Collected form C4 Explosive at all Flow Rates at

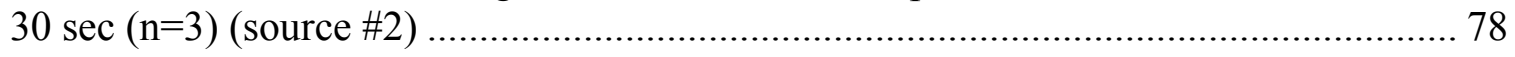

Table 8: 2E1H Mass Average Collected from C4 Explosive at all Flow Rates at $30 \sec (n=3)($ source\#3)

Table 9: DMNB Mass Average Collected from C4 Explosive at all Flow Rates at

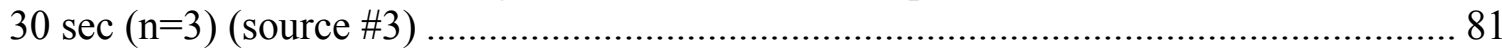

Table 10: Comparison of 2E1H and DMNB Mass Average Collected from C4............ 83

Table 11: 2,4-DNT Mass Average Collected at all Flow Rates at $30 \mathrm{sec}(\mathrm{n}=9) \ldots \ldots \ldots \ldots . . .85$

Table 12: 2,4-DNT Mass Average Collected form TNT at all Flow Rates..................... 87

Table 13: NG Mass Average Collected at all Flow Rates for $30 \mathrm{sec}(\mathrm{n}=9) \ldots \ldots \ldots \ldots \ldots . . . . . . . . .89$

Table 14: Comparison of Optimal Flow rates for 2E1H and DMNB Odorants .............. 92

Table 15: C4 Explosive Mass Average Collected at High Air Flow Rate at 60 sec........ 94

Table 16: C4 Explosive Mass Average Collected at High Air Flow Rate at 60 sec........ 95

Table 17: C4 Explosive Mass Average Collected at High Air Flow Rate at 60 sec........ 96

Table 18: Comparison of 2E1H and DMNB Mass Average Collected from C4 at all Locations at High flow Rate at $60 \mathrm{sec}(\mathrm{n}=3)$ 
Table 19: 2, 4-DNT Mass Average Collected at High Flow Rate $(\mathrm{n}=9)$........................ 101

Table 20: 2, 4-DNT Mass Average Collected form TNT at all Flow Rates.................. 103

Table 21: 2, 4-DNT Mass Average Collected from TNT at Low Flow Rate $(\mathrm{n}=3) \ldots . .104$

Table 22: Nitroglycerin Mass Average Collected from Double Smokeless Powder

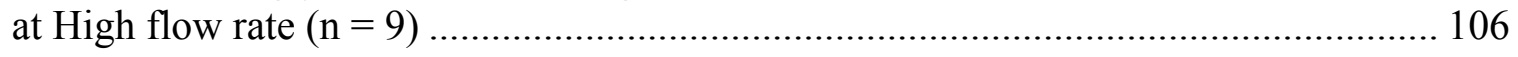

Table 23: Optimal Parameters for HSCS Collection of 2, 4-dinitrotoluene $(n=3) \ldots \ldots . .108$

Table 24: Average Mass Collected of 2E1H by Static Mode at Different Times .......... 113

Table 25: Average Mass Collected of DMNB by Static Mode at Different Times........ 115

Table 26: Static vs. Dynamic Collection of $2 \mathrm{E} 1 \mathrm{H}$ at 30 and $60 \mathrm{sec}(\mathrm{n}=3) \ldots \ldots \ldots \ldots \ldots \ldots . . . . . . . .117$

Table 27: Static vs. Dynamic Collection of DMNB at 30 and $60 \mathrm{sec} . . . \ldots \ldots \ldots \ldots \ldots \ldots \ldots \ldots . . . . . . . . . .118$

Table 28: Average Mass Collected of 2, 4-DNT by Static Mode at Different Times .... 121

Table 29: Average Mass Collected of 2,4-DNT (from 10g of single based smokeless

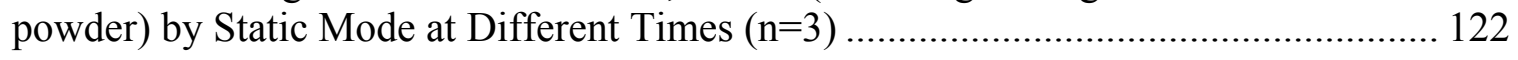

Table 30: Static vs. Dynamic Collection of 2,4 DNT at 30 and 60 seconds ................. 123

Table 31: Average Mass Collected of Nitroglycerin by Static Mode.......................... 125

Table 32: Static vs. Dynamic Collection of Nitroglycerin at 30 and 60 seconds ........... 127

Table 33: Average Mass Collected of 2E1H from C4 Explosive over a Month ........... 131

Table 34: Average Mass Collected of DMNB from C4 Explosive over a Month.......... 131

Table 35: Average Mass Collected of NG from Double Based Smokeless Powder

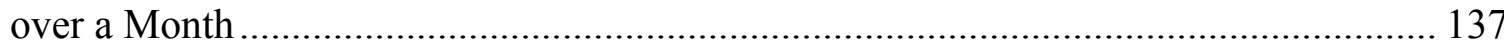

Table 36: Average Mass Collected of 2,4-DNT from Single Based Smokeless Powder

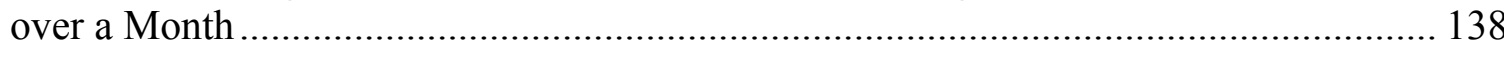

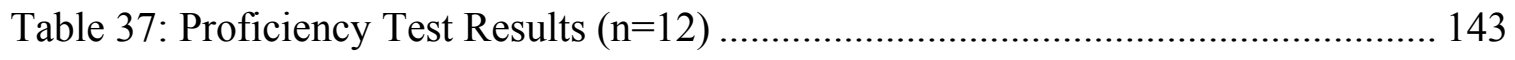

Table 38: Results of Field Evaluation of 3-week Aged Samples $(n=12)$..................... 144

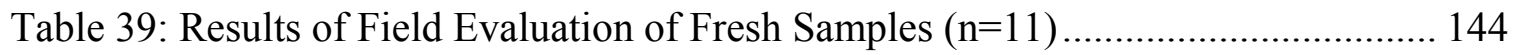


Table 40: Results of Field Evaluation of Blanks $(n=4)$

Table 41: Optimal Parameters for HSCS Collection of Explosive Volatiles

Table 42: Compound List, Including Retention Times, Masses, and a Summary of Results for Precision and Linearity. 


\section{LIST OF FIGURES}

FIGURE

PAGE

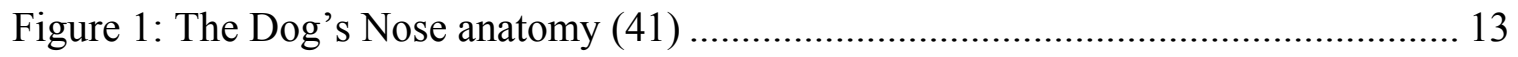

Figure 2: Solid Phase Microextraction Device (80) .................................................. 25

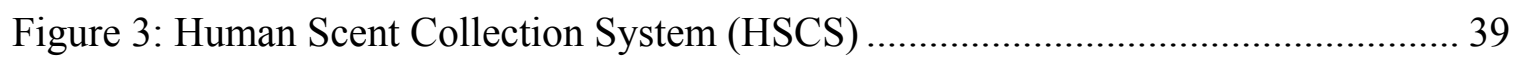

Figure 4: Linear Traverse for Round Duct $7.9 \mathrm{~cm}$-Diameter Approach........................ 40

Figure 5: HSCS Air Flow Velocity Measurements Taken by an Anemometer............... 42

Figure 6: HSCS Air Flow Volume at all Speeds in Liter/Minutes ............................... 43

Figure 7: HSCS System Setup for Explosive VOCs Collection................................... 44

Figure 8: HSCS Collection of 2E1H Chemical Compound...................................... 50

Figure 9: HSCS Collection of DMNB Chemical Compound ..................................... 51

Figure 10: Storage Containment Systems for Collected HSCS Samples ....................... 59

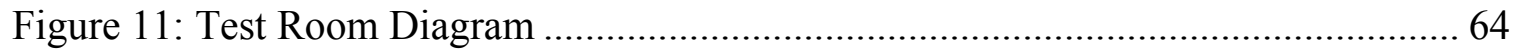

Figure 12: Headspace SPME-GC/MS of Tagged C4 Explosive from City of Miami

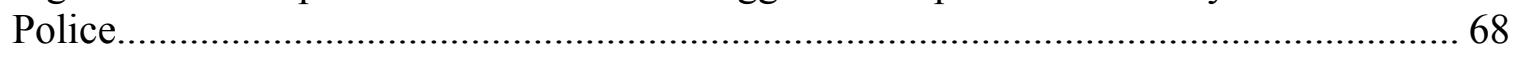

Figure 13: Headspace SPME-GC/MS of Tagged C4 Explosive from Miami .................. 69

Figure 14: Headspace SPME-GC/MS of Tagged C4 Explosive from the ATF .............. 69

Figure 15: Comparison of Extracted Ion Chromatograms from C4 Signature Odors

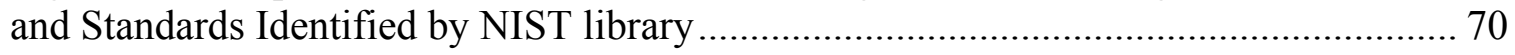

Figure 16: Comparison of 2,4-DNT GC/ECD Detection in Single Based Smokeless

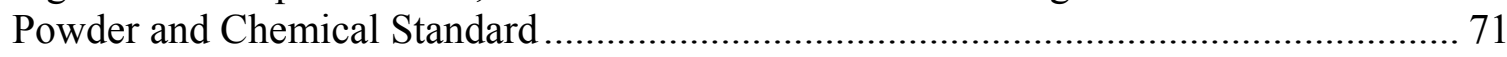

Figure 17: Comparison of 2, 4-DNT GC/ECD Detection in TNT and Chemical

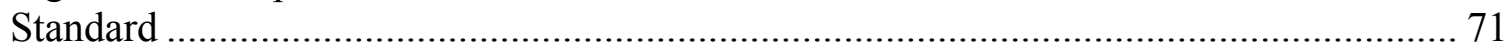

Figure 18: Comparison of NG GC/ECD Detection in Double Based Smokeless Powder and Chemical Standard. 72 
Figure 19: SPME Extraction Time Optimization of 2E1H............................................. 74

Figure 20: SPME Extraction Time Optimization of DMNB ………………………..... 74

Figure 21: Comparison Chromatogram of Optimal SPME Extraction Time (30 min) of 2E1H and DMNB at Different Temperatures......................................................... 76

Figure 22: HSCS Collection of C4 Explosive Using Different Flow Rates at $30 \mathrm{sec}$

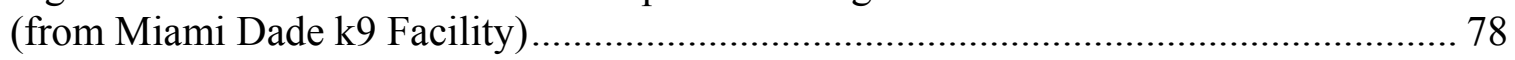

Figure 23: HSCS Collection of C4 Explosive Using Different Flow Rates at $30 \mathrm{sec}$

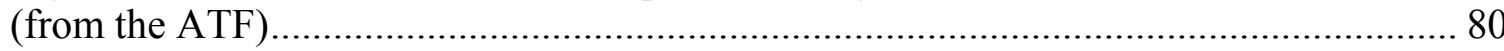

Figure 24: SPME Extraction Time Optimization of 2,4-DNT ……………………….... 84

Figure 25: Optimal Flow Rate for 2,4-DNT for $30 \mathrm{sec}$ Using the HSCS ........................ 85

Figure 26: HSCS Collection of TNT Using Different Flow Rates at 30 seconds ............ 86

Figure 27: SPME Extraction Time Optimization of NG .............................................. 88

Figure 28: HSCS Flow Rate Optimization of Nitroglycerin at $30 \mathrm{sec}$............................ 89

Figure 29: HSCS Collection of C4 Explosive at Low and High Flow Rates at $60 \mathrm{sec} . . . .92$

Figure 30: Semi-quantitative Analysis of C4 Volatiles (from source \#1) ........................ 93

Figure 31: Semi-quantitative Analysis of C4 Volatiles (from source \#2) ........................ 95

Figure 32: Semi-quantitative Analysis of C4 Volatiles (from source \#3) ........................ 96

Figure 33: Comparison of HSCS Collection of VOCs from C4 Explosive from all

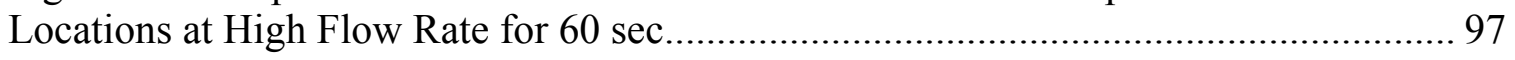

Figure 34: Headspace SPME-GC/MS of C4 Explosive Collected at High Flow Rate at $60 \mathrm{sec}$

Figure 35: 2, 4- DNT Sampling Time Optimization at High Flow Rate ........................ 100

Figure 36: Headspace SPME-GC/ECD of 2,4 DNT Collected at High Flow Rate at

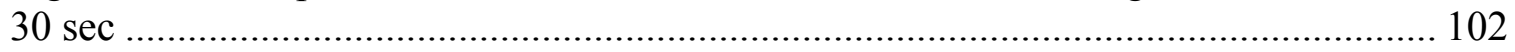

Figure 37: HSCS Collection of TNT Using Different Flow Rates for 60 seconds......... 103

Figure 38: HSCS TNT Sampling Time Optimization at Low Flow Rate........................ 104 
Figure 39: Headspace SPME-GC/ECD of TNT Collected at Low Flow Rate at 60 sec 105

Figure 40: NG HSCS Sampling Time Optimization at High Flow Rate....................... 106

Figure 41: Headspace SPME-GC/ECD of NG Collected at High Flow Rate at $30 \mathrm{sec}$. 107

Figure 42: (a) TNT and (b) Single Based Smokeless Powder Matrix .......................... 109

Figure 43: Static Collection of 2E1H from C4 Explosive at Different Times................ 112

Figure 44: Static Collection of DMNB from C4 Explosive at Different Times............ 114

Figure 45: Static vs. Dynamic Collection of 2E1H odorant at 30 and $60 \mathrm{sec} . . . \ldots \ldots \ldots . . . . .117$

Figure 46: Static vs. Dynamic Collection of DMNB Odorant at 30 and $60 \mathrm{sec} . . . . . . . . . . .118$

Figure 47: Static Collection of 2,4-DNT from Single Based Smokeless Powder at

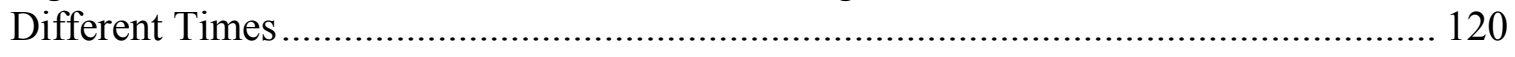

Figure 48: Static vs. Dynamic Collection of 2,4 DNT at 30 and 60 seconds ................ 123

Figure 49: Static Collection of NG Volatile from Double Based Smokeless Powder

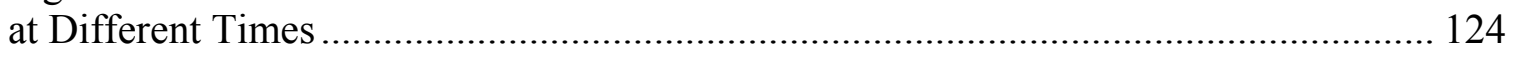

Figure 50: Static vs. Dynamic Collection of Nitroglycerin at 30 and $60 \mathrm{sec} . . . \ldots \ldots \ldots \ldots . . . . .126$

Figure 51: Persistence of 2E1H Target Odorant Overtime in the Tested Containers.... 130

Figure 52: Persistence of DMNB Target Odorant Overtime in the Tested Containers .. 130

Figure 53: Comparison of Different Storage Containers after One (1) hr Storage........ 133

Figure 54: Comparison of NG Samples Aged in Vials at one (1) hr, ........................ 135

Figure 55: Comparison of 2,4-DNT Samples Aged in Vials at one (1) hr, ................. 135

Figure 56: Persistence of NG Target Odorant Overtime in the Tested Containers ........ 137

Figure 57: Persistence of 2,4-DNT Target Odorant Overtime in the Tested Containers 138

Figure 58: Comparison of Different Storage Containers after one (1) hour Storage...... 140

Figure 59: Calibration Curves for 2E1H, DMNB, 2, 4-DNT, NG, and the Extracted

Ion Chromatograms for Selected Compounds.......................................................... 164 


\section{ACRONYMS AND ABBREVIATIONS}

\begin{tabular}{|c|c|}
\hline 2,4-DNT & 2,4-dinitrotoluene \\
\hline $2 \mathrm{E} 1 \mathrm{H}$ & 2-ethyl-1-hexanol \\
\hline AN & Ammonium nitrate \\
\hline ANFO & Ammonium nitrate fuel oil \\
\hline AP & Ammonium perchlorate \\
\hline ATF & Bureau of Alcohol, Tobacco, Firearms and Explosives \\
\hline $\mathrm{C} 4$ & Composition of $91 \%$ RDX and $9 \%$ plasticizers \\
\hline $\mathrm{CE}$ & Capillary electrophoresis \\
\hline COMPS & Control Odor Mimic Permeation System \\
\hline $\mathrm{CT}$ & Computed Tomography \\
\hline DART & Direct Analysis in Real Time \\
\hline DBP & Dibutyl phthalate \\
\hline DBSP & Double based smokeless powder \\
\hline DESI & Desorption electrospray ionization \\
\hline DDT & Deflagration to detonation transfer \\
\hline DMNB & 2,3-dimethyl-2,3-dinitrobutane \\
\hline DPA & Diphenylamine \\
\hline $\mathrm{EC}$ & Ethyl centralite (N,N'-diethyl-N,N'-diphenylurea) \\
\hline ECD & Electron capture detector \\
\hline EGDN & Ethylene glycol dinitrate \\
\hline GC & Gas chromatography \\
\hline
\end{tabular}




\begin{tabular}{ll} 
HMX & 1,3,5,7-Tetranitro-1,3,5,7-tetrazacyclooctane \\
HPLC & High performance liquid chromatography \\
IED & Improvised explosive device \\
IFRI & International Forensic Research Institute \\
IMS & Ion mobility spectrometry \\
IR & Infrared \\
LC & Liquid chromatography \\
LIBS & Laser induced breakdown spectroscopy \\
MS & Mass spectrometry \\
NMR & Nuclear magnetic resonance \\
NESTT & Nonhazardous explosives for security training and testing \\
NFSTC & National Forensic Science Technology Center \\
NQ & Nitroguanidine \\
OSAC & Organization of Scientific Area Committees \\
PAHs & Polycyclic aromatic hydrocarbons \\
PBX & Plastic bonded explosive \\
PDMS & Polydimethyl siloxane \\
PETN & Pentaerythritol tetranitrate \\
RDb & Parts per billion \\
ppm & Parts per million \\
\hline &
\end{tabular}




$\begin{array}{ll}\text { RI } & \text { Refractive index } \\ \text { SBSP } & \text { Single based smokeless powder } \\ \text { SCA } & \text { Surrogate continuation aid } \\ \text { SFE } & \text { Supercritical fluid extraction } \\ \text { SPME } & \text { Solid phase microextraction } \\ \text { SWGDOG } & \text { Scientific Working Group on Dog \& Orthogonal detector Guidelines } \\ \text { TATP } & \text { Triacetone triperoxide } \\ \text { Tetryl } & \text { Methyl-2,4,6-trinitrophenylnitramine } \\ \text { TNT } & \text { 2,4,6-trinitrotoluene } \\ \text { TOF-MS } & \text { Time of flight mass spectrometry } \\ \text { UDC } & \text { Universal detector calibrant } \\ \text { UV } & \text { Ultraviolet } \\ \text { VOC } & \text { Volatile organic compound } \\ \text { WWI } & \text { World War I }\end{array}$




\section{INTRODUCTION}

The enhancement of explosive canine detection methodologies is a top security challenge as a result of the rapid evolution of explosive mixtures employed in national security threats. Explosive compounds are rarely found in a pure state, frequently they are the result of the combination of multiple materials including: stabilizers, tagging agents, plasticizers or other additives and usually commercial and military high explosives have low vapor pressures which make them very hard to detect. Currently, there are many explosive training aids being used to train detection canines [1]. However, to date an optimal training aid has not been created and the development of a training aid kit suitable for the ever evolving explosive recipes encountered in military operations is necessary to provide a robust and efficient pathway to train canines for explosives detection.

The aim of the current research is to provide an evaluation of the human scent collection system (HSCS) as a collection device for volatile organic compounds (VOCs) emanating from real explosive material for the creation of training aids for explosive detection canine teams. The research involves combined laboratory and field testing components through the use of HSCS as a viable method for the preparation of training aids to be tested with certified canine teams to evaluate their capabilities to produce an alert to a sample collected via dynamic airflow collection. The analytical approach will consist of the headspace evaluation of the collected explosive odor through solid phase micro-extraction (SPME) followed by analysis using both gas chromatography-mass spectrometry (GC/MS) and gas chromatography-electron capture detection (GC/ECD) in

order to identify the volatile chemical profile of each explosive odor class at room 
temperature. All samples will initially be collected onto a gauze pad using the HSCS as the method of collection. Experiments will follow a previous evaluation of the signature odor chemicals of four explosive families: 2,3-Dimethyl-dinitrobutane (DMNB) representing the nitro-alkanes, nitroglycerin (NG) representing the nitrate-esters, 2,4dinitritoluene (2,4-DNT) representing the nitro-aromatics, 2-ethyl-1-hexanol (P) representing the plasticizer commonly found in conjunction with trinitrotriazacyclohexane (RDX) and 1,3,5,7-Tetranitro-1,3,5,7-tetrazacyclooctane (HMX) representing the nitro-amines [1-4]. Additional experiments include: optimization of HSCS airflow, sampling time, a comparison of the different methods of collection: static vs. dynamic. Also to establish the persistence of volatiles and the ideal storage containment for the collected aids as well as field testing.

The present project entails the creation of training aids using a dynamic airflow system which could be used for military applications, and represents a novel and viable way of collecting newly emerging explosive odorants in combat areas where new explosives are being used and can then be brought back for training purposes. Currently, real explosives are required to perform maintenance training of canines in the field which causes difficulties with the transportation and storage of these explosives, reduces the frequency of training, and of course represents a great risk for both: the canine and the handler. The current project will demonstrate that the use of these non-hazardous training aids made from volatiles collected using the HSCS from real explosives will not diminish canine's ability to detect explosives. On the contrary, these training aids will provide efficient canine training scenarios in which real explosive material is no longer necessary. 


\section{EXPLOSIVES OVERVIEW}

The term explosive is generally used in reference to a wide range of energetic materials that can react to produce heat, light, and gas. Explosives are combinations of oxidizers and fuels that are capable of high rates of reactions called either deflagrations or detonations [5]. The potential energy stored in an explosive material may be chemical energy (such as nitroglycerine), pressurized compressed gas (such as gas cylinder or aerosol), or nuclear (such as fissile isotopes of uranium-235) [5].

\subsection{Classification of Explosives}

\subsubsection{Low and High Explosives}

Explosives can be divided into two categories on the basis of how they release energy: low explosives (LE) and high explosives (HE). Low explosives (LE) require confinement to be effective. Their rate of decomposition is propagated by a flame front (deflagration) at less than the speed of sound. Low explosives (LE) include propellants which undergo rapid combustion without detonation and the resulting gas produced is used for propulsion purposes such as that to propel a bullet or a missile and pyrotechnics that contain a fuel and an oxidizer to produce a lot of energy to create a flame and light [5]. Examples of propellants include black powder and smokeless powder. Smokeless powder contains nitrocellulose (NC) and according to their chemical composition can be divided in three groups: single base powder (containing only $\mathrm{NC}$ ), double based (NC, nitroglycerine), triple based (NC, nitroglycerin, and nitro guanidine) [5,6,7 ].

High explosives (HE) will function without confinement and are characterized by the extreme rapidity with which decomposition occurs; this action is known as 
detonation. When initiated by a blow or shock, high explosives will decompose almost instantaneously $[5,6,8]$. According to the level of sensitivity to stimuli, high explosives can be divided into three subcategories: primary, secondary, and tertiary $[5,6]$. Primary Explosives such lead azide, are extremely sensitive to ignition by heat, friction, spike, impact, flame, or electrostatic discharge and, as a consequence are extremely dangerous. Very small quantities can undergo deflagration to detonation transfer (DDT) and are used as initiators to detonate secondary explosives [5]. Almost all detonators contain primary explosives. Secondary Explosives generally are far less sensitive than primaries and are used to intensify detonation. These types of explosive materials are the more commonly used in bulk and contain nitro aromatics, nitro amines, and nitrate esters that can be casted or plasticized. Cast explosives use 2, 4, 6-trinitrotoluene (TNT) such as tetratol. Plasticized explosives like C-4 use additives such as 2-ethyl-1-hexanol and odorizing taggant (volatile chemicals used to mark explosives for future identification) chemicals such as 2, 3-Dimethyl-dinitrobutane (DMNB) [5,6]. Both of these components have been identified as the dominant headspace chemical representatives of explosives [5]. Detonating a secondary explosive requires higher energy levels created by another explosion, usually created by a primary explosive. For example; (TNT) is commonly used in mixtures of explosives because of its stability, moisture resistance, and insensitivity to friction [5,6]. Tertiary Explosives (also called blasting agents) usually require the initiation from a secondary explosive to cause detonation and are the less insensitive type of explosives. Tertiary explosives are based on ammonium nitrate (AN) and ammonium perchlorate [9]. 
Depending on the manner of production and expected usage, explosives can also be classified as military (i.e., artillery), commercial (i.e., mining, demolition purposes), or improvised [6]. Military explosives refer to primary explosives that do not require other components to make them explode such as TNT. Military explosives typically contain oxygen carried by the nitro functional group, $\mathrm{NO}_{2}$. This functionality may be attached to oxygen $\left(\mathrm{O}-\mathrm{NO}_{2}\right)$ as in the nitrate- esters such as $\mathrm{NC}, \mathrm{NG}$, or PETN or to a carbon $(\mathrm{C}-$ $\left.\mathrm{NO}_{2}\right)$ as in the nitro-aromatics such as TNT, or to a nitrogen $\left(\mathrm{N}-\mathrm{NO}_{2}\right)$ as in the nitroamines like RDX [5]. Commercial explosives refers to those utilized for blasting operations in civil and mining projects such as dynamite, and improvised explosives which are manufactured in clandestine laboratories like peroxide-based improvised explosives which are an emerging threat for terrorist activity because they include chemical constituents easily found in any home or local community and can be as effective as manufactured explosives in many applications. One example of a peroxide containing explosive is Triacetone-triperoxide (TATP) [5]. Regardless of type, all are extremely hazardous because of their sensitivity and difficulty to be handled in a safe manner.

\subsubsection{Chemical Components of Explosives}

Substances that are explosive in nature contain molecular groups with explosive properties. These substances generally contain oxygen, nitrogen, and a fuel or an oxidizable element such carbon and hydrogen. The oxygen is usually attached to a nitrogen as in nitro groups $\mathrm{NO}, \mathrm{NO}_{2}, \mathrm{NO}_{3}$, and in the event of a chemical reaction the nitro group separates and combines with the fuel component. Some exceptions are the 
azides, such as lead azide $\left(\mathrm{PbN}_{6}\right)$, in which no oxygen is present $[5,6,7]$. According to their chemical nature, explosives have been classified by family groups that include: nitro alkanes, nitro amines, nitro aromatics, nitrate esters, peroxides, and acid salts [5].

The nitro-alkane group is distinguished by the group $\mathrm{C}-\mathrm{NO}_{2}$ attached to an aliphatic carbon back-bone. The most common example related to this group is 2, 3dimethyl-2, 3-dinitrobutane (DMNB) which is a volatile organic compound used as a detection taggant for explosives [6]. The nitro-amine group is characterized by the presence of nitrogen attached to a nitro molecule: $\mathrm{N}_{-} \mathrm{NO}_{2}$. Examples of explosives belonging to this group include HMX (high melting explosive; octahydro-1,3,5,7tetranitro-1,3,5,7 tetrazocine), RDX ( hexahydro-1,3,5-trinitro-1,3,5 triazine, which is also known as cyclonite), nitroguanidine, and tetryl. The nitro-aromatic family refers to those substances containing molecular group $\mathrm{C}-\mathrm{NO}_{2}$ attached to an aromatic ring. The best known nitro-aromatic compound is the explosive TNT (2, 4, 6-trinitrotoluene). The nitrate-ester group is identified as $\mathrm{C}-\mathrm{O}-\mathrm{NO}_{2}$ in which the nitro group is bonded to an oxygen atom. Examples include nitrocellulose (NC), nitroglycerin (NG), PETN (Pentaerythritol tetranitrate), and EGDN (ethylene glycol dinitrate) [6]. Peroxides refer to compounds in which two oxygen atoms are linked together by a single covalent bond CO-O-C. The unusual weakness of the -O-O- bond is most likely as a result of the high electronegative character of the oxygen atoms. For this reason, peroxides are extremely prone to violent decomposition initiated by heat, mechanical shock, or friction. The acid salts are the result of a binary combination of an anion with a cation such ammonium nitrate $\left(\mathrm{NH}_{4} \mathrm{NO}_{3}\right)$. Ammonium nitrate (AN) is the least expensive source of oxygen available for commercial explosives or to be used in conjunction with fuels (ANFO) or 
with other explosives such TNT and nitroglycerin [9]. Other acid salts used for pyrotechnics can be formed with chlorates $\left(\mathrm{ClO}_{3}\right)$ and perchlorates $\left(\mathrm{ClO}_{4}\right)$ mixed with sodium $\left(\mathrm{Na}^{+}\right)$or potassium $\left(\mathrm{K}^{+}\right)[7]$.

\subsubsection{Other Constituents of Explosives}

Low explosives such as propellants contain certain additives necessary to modify the burn rate of the explosive. These additives can be classified according to their function. A given additive can be used for more than one function such as carbamite which is used as a stabilizer, plasticizer, and coolant [5].

Additives that soften the powder granules and reduce the need of a solvent are called plasticizers. Some examples include: carbamite, dinitrotoluenes such 2, 4 DNT and 2ethyl-1-hexanol $(2 \mathrm{E} 1 \mathrm{H})$. Stabilizers are used to increase the shelf life of the explosive material by removing nitric acid during the decomposition of nitrated energetics. The stabilizers most commonly used are carbamite, diphenylamine and its nitrated derivatives. Taggants which are volatile chemicals used to mark explosives for further identification such 2, 3-dimethyl-2, 3-dinitrobutane (DMNB) [6]. Deterrents like dinitrotoluenes, phthalates, and ethyl centralite are used to coat the powder to reduce the initial burn rate. Coolants are yet another group of constituents that lower the temperature and reduce the initial burn rate such as dibutyl phthalate, carbamite, dinitrotoluenes, and methyl centralyte $[5,6,9]$. 


\subsection{Detection of Explosives}

Significant advances in operational instrumentation have allowed for enhanced detection of explosives. Detection techniques are focused on either bulk explosives or traces of explosives. Detection of bulk explosives is carried out either by imaging characteristics of the explosive device or by detection of the explosive itself. Trace detection utilizes either emitted vapors from the explosive or explosive particles deposited on surfaces. Some of the techniques used for bulk explosives include X-ray systems such as computed tomography (CT) that allows the characterization of materials by density, atomic number, and texture; and can be applied to personnel, luggage, large and small cargo, and vehicles $[6,9,10]$. X- ray diffraction $(\mathrm{XRD})$ provides high specificity and very good spatial resolution for screening objects such as boxes and larger luggage. Neutron- based technologies with emitted gamma rays, which readily pass through most common materials (including metal), can be used in a wide variety of explosives detection applications including: vehicle, small cargo, and baggage screening. Microwave and ultrasonic technologies have also been applied for analysis of liquid explosives during opaque bottle screening [9].

Trace explosives are commonly identified by using mass spectrometry (MS) as a result of its specificity in identifying substances and the speed of the analysis. Mass spectrometry (MS) separates and analyses the chemical composition of a substance according to its mass-to-charge $(\mathrm{m} / \mathrm{z})$ ratio. Some forms of MS used for detection of explosives include: quadrupole, ion trap, time-of-flight (TOF), and tandem based techniques (MS/MS). High-performance liquid chromatography (HPLC) instruments using UV absorbance or refractive index (RI) detector, supercritical fluid (SCF), infrared 
spectroscopy, and capillary electrophoresis (CE) with luminescence have long been used for explosives characterization as well [9].

Coupled techniques such as gas chromatography-mass spectrometry (GC-MS) have also been utilized. These systems combine the separation ability of gas chromatography with the sensitivity and specificity of a mass spectrometer that is capable of detection of a wide range of explosives. Gas chromatography coupled to an electron capture detector (ECD) has proven to be the best and most sensitive to detect electronegative species such as nitro-groups and chloride [6,8,9 ]. Approaches such as ion mobility spectrometry (IMS) characterize a sample through the mobility of ions within the gas-phase of the instrument when an electric field is applied. The recent development of a new IMS inlet in which solid phase microextraction (SPME) can be used for the analysis of volatile compounds present in headspace systems has been used for identification of different explosive odor signatures [11]. Actually, miniaturizations of IMS devices have been widely used to detect explosives when checks are performed on passengers, baggage, vehicles, and containers $[9,10]$.

Currently, laser techniques such as laser-induced breakdown spectroscopy (LIBS) have also been introduced for the identification of a wide range of explosive compounds [12]. Additional ambient ionization techniques such DART and DESI have shown to be effective for the detection of trace explosives in situ from a variety of surfaces $[13,14]$. Recently, DESI was coupled to a portable miniature mass spectrometer that can be handled into the field [15].

New trends of portable sensors include chemical sensors that produce a chemical reaction with an explosive vapor, leading to an observable product such as a change in 
color or conductivity [16]. Electrochemical sensors have been developed to respond to redox substances; therefore electrochemical detection is possible using the redox properties of nitro-aromatic explosive substances $[10,17,18]$. Electronic noses have also been used as chemical sensors in which each sensor interacts with vapor concentrations in different ways to eventually allow the recognition of the target compound [17]. Actually, the use of biological detectors including dogs and rats, has been of great acceptance for their potential application to detect volatile compounds of forensic significance in field-based detection systems [19].

\subsection{Biological Detectors}

In the field of forensics, law enforcement, and rescue teams, biological olfactory systems have been utilized because they possess the ability to detect a wide range of volatile compounds from explosives, narcotics, humans, and cadavers. Not only dogs (Canis familiaris) have been trained to detect specific substances, but also other species including rats, insects, and dolphins $[19,20]$. Ongoing studies are focused on birds and elephants as possible future detectors of explosive material. However, to date, canine detectors have been the traditional method utilized by military and law enforcement agencies for detection purposes because of their capabilities including, mobility, rapid response, reliability, selectivity, and availability to work in open areas and under different environmental conditions.

According to recent studies of biological systems for detection, rats have shown their ability to detect different concentration of TNT in air [21]. These detectors provide the benefits of low cost, small size, and light weight [22]. However, they do not work 
well in open spaces as canines do. Insects are very sensitive, cheap to reproduce, and easy to condition them to detect target odorants [23]. For example, honeybees have been trained by injecting them into their feeder with trace amounts of a target compound such 2, 4 DNT. As a result, the insect will seek sources of food that contain the same compound [24]. However, as opposite to dogs, insects are highly affected by environmental conditions and also have a short lifespan [24]. Other investigations have shown that dolphins are trainable mammals because of their great intelligence and highly developed sonar capability. These mammals have been efficiently trained to detect submerged vehicles in the ocean and sea mines in cluttered shallow-water environments where military electronic devices are absolutely useless [25]. Dolphins, as well as dogs, offer the advantage of being able to establish social relationship with humans. Of all of the biological organisms utilized for detection, canines are still the most valuable tool primary for its olfactory capabilities, easy access and training. In the past few years, canines have been trained in different areas and therefore become an important tool in forensic investigations such as detection of ignitable liquid residues and explosives or their chemical precursors, $[3,26,27]$. In the same manner, narcotic canines have been successfully trained for the detection of illicit drugs including heroin, cocaine, marijuana, methamphetamines, and their derivatives. [28,2,29]. Canines also have shown to be scent discriminators since they can alert on the scent of a specific person after being given a sample of that person's scent [30]. Furthermore, canine teams have demonstrated their ability to identify human scent even in the presence of other odorants. Recently, canines were able to locate individuals who have been in contact with improvised explosive device (IED) components recovered at a post-blast scene [31]. In addition, canines that 
detect human remains (also called cadaver dogs) have shown to be effective in the detection of buried human remains (fresh and at various stages of decomposition) at different depths $[30,32]$. As a consequence of the acute sensitivity of the canine olfactory system, canine training has been extended and actually enabled dogs to detect guns, cellphones, pipeline leaks, currency, contraband food, mold, and even cancer [33 -39].

The successful use of canines for the detection of volatiles of forensic interest has been demonstrated. However, they offer some disadvantages such as high cost of training, medical care, and other regular maintenance expenses. Also canine responses are highly dependent on their training, attention span, limited duty cycles as well as handler's judgment. In comparison with instrumental detectors, the actual operational employment of biological organisms represents a great advantage because of their mobility capacities, rapid response, and wider application for forensic purposes.

\subsubsection{Canine Olfaction}

Olfaction is a sense regulated by specialized sensory cells located in the epithelium of vertebrates and the primary sensory system used for social interaction, to locate food, detect predators, and to locate mates. The well-developed canine olfactory system has the remarkable capacity to detect and discriminate odorants from a vast range of odorant molecules [40].

The dog's nose has a nasal plane at the end that is hairless and black in which two nostrils are located. Internally, the nose is divided into two chambers separated by the nasal septum. Within each of the cavities are the turbinates and the frontal sinuses. The turbinates consists on the epithelium formed by the mucus membrane which contains 
supporting cells that serve as a filter to trap bacteria and to keep dirt from entering the nasal cavity, mucus cells to provide moisture, and scent cells or receptors that trap the odors [41]. During sniffing, the surface area of the epithelium freely allows the entrance of air. This air is warmed and humidified as it passes through the turbinates to eventually be trapped in the mucus layer and processed by the sensory cells or olfactory receptors into electrical signals to be detected by the brain [41,42] (see Figure 1).

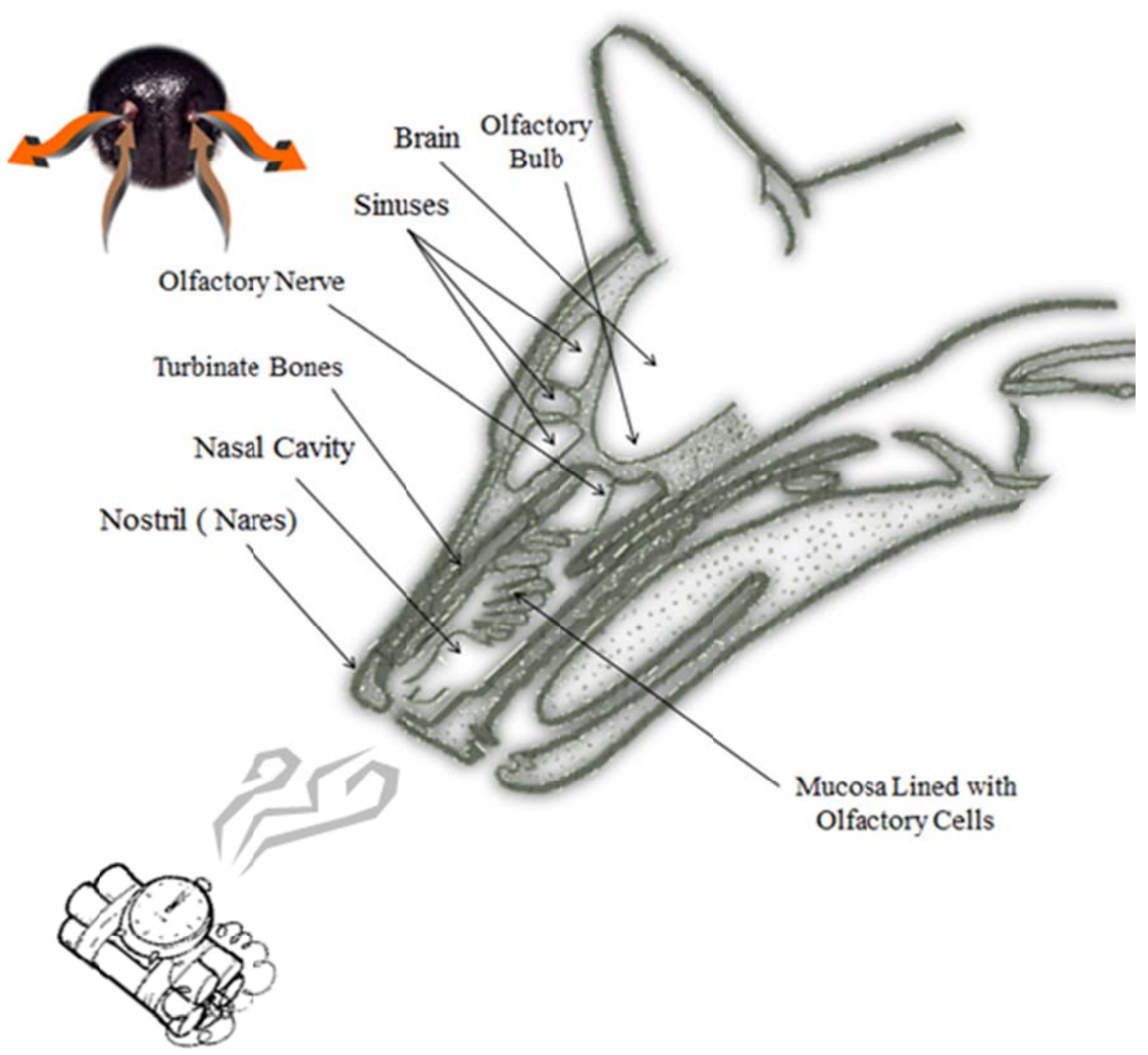

Figure 1: The Dog's Nose anatomy (41) 
Genetic studies reveal that one of those receptors can recognize multiple odorants and that a single odorant can be detected by multiple receptors, and different odorants are recognized by different combinations of olfactory receptors. This clearly indicates the high potential for recognition and discrimination of odorants in canine olfactory system. In addition, the canine brain has a tremendous number of olfactory cells. The percentage of the dog's brain specialized in olfactory activity is actually 40 times larger than humans with a sensitivity which is 1,000 times greater than that found in humans. [43, 44]. In humans the area of the epithelium is $5 \mathrm{~cm}^{2}$ whereas the dog has a surface of $150 \mathrm{~cm}^{2}$ and 250 million olfactory cells [45]. Furthermore, dogs have the ability to track directions [46], have long-term olfactory memory, and can discriminate odorants from mixtures. For these reasons, there is increasing interest in the dog's incredible sense of smell for detecting volatile compounds associated with forensically significant substances.

\subsubsection{Canine Detection and Explosive VOCs}

Despite the significant advances in operational instrumentation, limitations exist. These systems require not only constant expansion of the instrument library as the threat from explosives changes, but also can only detect minute particles of explosive materials. Instrumental limitations in detecting explosive vapors are particularly attributed to the very low vapor pressures of most explosives which make them very difficult to measure. For this reason, canine teams are still considered to be one of the most sensitive, accurate, reliable, fast, and effective technologies employed by law enforcement personnel worldwide. 
For their enhance mobility and olfactory capacity canines have been used for explosive detection since World War II [1]. Canine training for detection and location of specific people or substances of interest has become of vital importance for legal investigations for decades [ $3,9,26,27,30]$.

Canine training refers to the development of desirable responses through the learning process, but to understand the training of detector dogs it is important to discuss some important concepts like stimulus which is a condition or an external influence or activity that produces a response such as a change in behavior. Animals have instinctive or innate responses to stimuli. A behavior is instinctive if it is performed without being based upon prior experience, in other words; in the absence of learning. A behavior is innate when is related to a specific learning pattern in which canines are imprinted. Imprinting is then defined as learned stimuli that produce an innate behavior as a response [47]. Canines are capable of being imprinted on single or multiple odorants. Dogs are trained to sit, scratch, bark, or lie down near the object where they have detected the odorants they were imprinted to. The change in bog behavior is called positive response or alert. During training, dogs are rewarded after they perform an expected behavior. In this way, the dog learns that a reward (i.e. toy) is delivered, if he acts as expected. In this learning process, the dog only receives the reward after he gives a positive response during a search [48]. Once a canine is imprinted on specific materials, the canines must perform training on a regular basis to maintain a satisfactory level of detection in addition to being exposed to a variety of environmental conditions suitable to expected operational needs. [49]. Actually there is no standardized breed for canine explosives detection, but detector dogs are usually German shepherds, Belgium Malinois, 
Labrador retrievers, and hound dogs. In order to be selected as detector dogs, canines have to show certain characteristics such as obedience to the handler, motivation and quick response, and the desire to track and retrieve the reward [48,50].

Through effective canine training, in the last decade canines have been trained to detect flammable and ignitable liquid residues and their alert has proven to be admissible as evidence in courts [51]. Plastic explosives were originally developed for convenient use in military demolitions but recently have been used by terrorist. These explosives contain plasticizers such as 2-ethy-1-hexanol which are added in small quantities because they are inert and would degrade explosive output. Plasticizers are more volatile than the explosive component; therefore for detection purposes, authorities rely on key vapor signatures for canine training.

The determination of the chemical signature to which canines are actually alerting to has relied in the accurate definition of a common odorant within different explosive mixtures. Studies in this area play a pivotal role to the efficient use of training odor mimics in practical field applications. Research conducted in the area has identified common dominant odor chemicals emanating from explosives [1,2,52,53 ]. In turn, these key odor chemicals have provided positive responses from explosive dog teams. For example; 2, 4-dinitrotoluene and 2-ethyl-1-hexanol have been reported as important odor chemicals for canine detection of cast and polymer containing explosives $[1,2,52,53]$. The extraction of odor signature compounds of smokeless powders and plastic explosives with SPME-IMS techniques has enabled the detection of target odorants complementing canine detection and allowing a means of standard calibration with analytical instruments [3,54 ]. Instrumental evaluation of these compounds has led to improvements in training 
aid mimics for canine explosive detection teams. An odor mimic can be defined as an imitation or simulative that incorporates the dominant compounds found in the headspace of the actual compound and can be utilized for biological and instrumental detectors. Previous studies have determined the dogs limits of detection for cyclohexanone (one of the most abundant chemicals present in the headspace of $\mathrm{C} 4$ explosive material [53] and nitroglycerin to be in the parts per billion (ppb), and the sensitivity to DMNB (a detection taggant) to be much greater at 500 parts per trillion (ppt) [55 ].

Parallel studies suggest that an entire vapor fingerprint is primarily involved for canine detection of a representative explosive material [52,53]. For this reason, it is crucial to understand the importance of explosive VOCs because numerous volatile organic compounds are possible including plasticizers (phthalates, TNT, 2-ethyl, 1hexanol), and stabilizers (including diphenylamine), since it is hypothesized that dogs use the most abundant chemicals present in the headspace to locate concealed explosives. $[1,2,11,52,55,56]$.

\subsubsection{Training Aids}

In the past few years, some canine training aids have been developed by using inert substances that mimic the odor signatures of explosives. One example includes the nonhazardous explosives for security training and testing (NESTT), but these provide inconsistent results since canines have had difficulty in locating the NESTT aids [1]. The Army's Military Working Dog Program authorizes commercial dynamite (gelatin and ammonium nitrate), military dynamite, TNT, smokeless powder, $\mathrm{C} 4$, detonating cord, potassium chlorate, and sodium chlorate as training aids [56]; but these aids require the 
use and exposure to the real explosive material. To circumvent these challenges, further studies in the area of volatile odorants has led to the development of surrogate continuation aids as observed in the International Forensic Research Institute (IFRI) Prototype Surrogate explosives kit. The continuous testing of these aids further advances and strengthens the technology of control odor mimic permeation system (COMPS) and paves the way for a standardized canine training aid kit. Control odor mimic permeation system (COMPS) is a new technique in which target odorants can be stored inside a permeable package such as low density polyethylene bags and sealed within a nonpermeable membrane like metallized polyester and/or glass. Control odor mimic permeation systems (COMPS) are individualized light weight units in which no external operation system is necessary. They offer the benefit of being disposable and relatively inexpensive. Control odor mimic permeation system (COMPS) can provide an odor mimic or an imitation to that of the actual volatile compound or compounds present in an explosive material, which can then be utilized for canine training purposes [57]. The development of an optimized explosive mimic has shown the combination of six odors with positive results for imprinting detection canines. The comprehensive odor kit uses a single based smokeless powder with a detectable level of 2,4-dinitrotoluene, and a double based smokeless powder with a detectable level of nitroglycerine, 2-ethyl-1-hexanol, DMNB, ethyl centralite, and diphenylamine shown to be accurate mimics for TNTcontaining explosives, NG-containing explosives, plastic explosives, tagged explosives, and smokeless powders, respectively [58].

Recently, a universal detector calibrant (UDC) has been proposed for the calibration of canine detectors performance. One (1)-Bromooctane (1-BO) was selected as the UDC 
since it fulfilled all the mandatory and desirable qualities for acceptable UDC. These desirable qualities include factors such as: low hazard level for both canine and handler, not be a target odorant, be easy detected, allow permeation at a constant rate, have enough volatility for rapid detection, thermal stability, potential for daily use, easy access, and low cost. The goal of this UDC with these characteristics is to ensure that canine detectors are working within acceptable limits. Moreover, the UDC could potentially provide vital information regarding the achievements of the canine including the number of alerts, misses, and error rate to produce optimal results regarding accuracy and reliability of training and to make the canine detector more comparable with analytical instruments. It was determined that 1-BO is not a dominant odor compound used by biological detectors and canines could be successfully imprinted and capable of searching and alerting on 1-BO with a $100 \%$ proficiency, although its practical use in field operations is still in undergoing research [59].

Training aids focused on peroxide explosives have been developed. These aids utilize cotton balls that have been spiked with very low concentrations of diluted TATP and HMTD or through adsorption of these explosive vapors onto a cotton ball to be presented to the canines in close proximity for a certain period of time $[60,61]$. These compounds are so complex, they require special handling conditions such as refrigeration or other specialized storage system since they are highly volatile and unstable, therefore presenting a risk to the canine as well to the handler.

In an effort to implement the IFRI surrogate explosive kit, a non-hazardous mimic aid for TATP has been designed which can be utilized by the use of COMPS or pads soaked in acetone or hydrogen peroxide. These compounds have to be always used in 
combination as the use of acetone or hydrogen peroxide alone as a training aid can provide high false alerts to other common containing compounds [59 ].

The selection of the proper storage containment system is crucial for the maintenance of the integrity of canine training aids and to prevent cross-contamination of odorants. Currently, a variety of containment systems including glass and plastic containers are utilized for training aids storage. International Forensic Research Institute (IFRI), for example has designed an optimal containment system using three levels of containment to provide the lowest potential of contamination and to guarantee the preservation of the explosive vapors [59]. In general, an optimal training aid is one that represents no risk to the dog and the handler, is long lasting, difficult to contaminate and requires no special conditions.

One of the greatest challenges in explosive canine detection work is the optimal selection of training aids. The complexity lies in the wide range of explosives within each category combined with the variety and sophistication of explosive formulations throughout the world. In response to this variation, the Scientific Working Group on Dog and Orthogonal Detector Guidelines (SWGDOG) has developed the best practice guidelines to improve the performance, reliability, and courtroom acceptance of detector dog teams to provide a source of standardization to local law enforcement and homeland security.

\subsubsection{Canine Detectors and the Law}

The science underlying canine olfactory detection capabilities has been the target

of intense research and currently courts are asked to rule on the admissibility on a variety 
of canine evidence. The acceptance of using canine searches as a technique for detection purposes has been scrutinized as any other scientific method presented as evidence in the judicial system. Some history behind the acceptance of scientific evidence by The United States Supreme Court refers to the landmark case Frye v. United States in 1923 [62] which stated that a scientific technique can be accepted if it has gained general acceptance within its particular field. Later on, in the Daubert v. Merrell Dow Pharmaceuticals, Inc. case in 1993 [63] the Supreme Court estimated that the Frye standard in law courts was no longer sufficient as general acceptance of scientific evidence. The Daubert test named the judges as "gatekeepers" of expert evidence. This test also incorporated that evidence can be accepted if the technique used is feasible and has been tested, has been subject to peer review, shows the potential levels of error, and if the technique has been generally accepted within the scientific community. Eventually, the Supreme Court in 1999 in Kumho Tire, Inc. v. Carmichael case established that the criteria for the acceptance of scientific evidence presented in Daubert should also apply to any kind of expert testimony including testimony based on knowledge and experience in canine training [64].

Since the beginning of the $20^{\text {th }}$ century, the use of canine searches as evidence in court of law has existed. In Hodge v. State [65], the testimony regarding tracking dogs was admissible as evidence to be presented to the jury. Later, in State v. Hall [66], canine evidence was admitted in court as there was enough information related to training, records of canine performance and handler experience. As a result of terrorism and criminal activity, many agencies worldwide use canines for detection of substances and apprehension of persons. The purpose of the law enforcement canine is to determine the 
probable cause for a search. However, resistance regarding their use has grown in courts from cases related to drug traffic or other illegal material seizure.

Most courts recognize the scientific validity of canine's ability not only to detect and discriminate odorants but also to produce reliable identification in different situations (i.e., tracking) [67]. They have also recognize the non-destructive nature of canine search, and the importance to provide fast means for detection of illegal substances and dangerous material in those places where rapid and reliable detection is needed (i.g., airports, luggage, vehicles). Since the US Supreme Court addressed the Fourth Amendment that "a canine sniff of an inanimate object is not a search" [68], and is not a matter of discrimination or against the civil rights, but instead if a reasonable suspicion exist, then the use of a dog to sniff is not considered a search.

In 2005, the U.S. Supreme Court according with the case Illinois v Caballes [69] stated that police had "probable cause" to search any individual's vehicle if the police detector canine, provides an alert for the presence of an illegal substance ( by sitting, barking or providing any signal the dog has been trained to alert). In addition, courts have recognized that a dog must be reliable for an alert to have this consequence. Such reliability is established by showing that the canine has the proper training, qualifications, complete field records of the canine performance, and certification by an established organization. The effectiveness of canine training has been demonstrated in a case in Arizona in which a conviction was affirmed using evidence from an explosive detector dog (EDD) that provided an alert when sniffed the locker of an individual who was under surveillance for suspected criminal activity involving explosives [70]. The court 
established that the alert and other evidence was enough to issue a warrant where explosive residues and related tools were found.

In an effort to establish the scientific validity of canine detectors, some institutions and agencies provide training and certifications. That is the case of the International Forensic Science Research Institute (IFRI) at Florida International University and the National Forensic Science Technology Center (NFSTC) which are recognized nationwide and offer training certifications in many areas including drugs, explosive, arson, and currency. A Certification is free of charge because of government support and valid for one year from the date it is issued [71]. Other agencies such The Bureau of Alcohol, Tobacco, Firearms and Explosives (ATF) offer EDDs to other Federal, State, local and foreign law enforcement agencies. These dogs must pass rigorous tests where they must successfully detect different explosives odors (according to the protocol provide by the agency) [72]. These canine detectors are required to meet highest standards performance in search and detection. However, many agencies require different proficiency rates. The American Police Work Dog Association requires a minimum of $91.6 \%$ [73], IFRI/NFSTC require $90 \%$ or greater [74] while others, such as the ATF and the U.S. Customs Service, request a 100\% proficiency for certification [75].

Today the role of the canine handler is expanding as a result of an increase demand for canine detectors in ports, airports, and many other public places. Because of high demand; the handler's ability to interpret the canine's behavior and responsiveness are of crucial importance in evaluating the validity of canine evidence. Despite previous comments regarding handler beliefs influencing canine performance [76], The Supreme Court's acceptance of canine evidence has been affected in the Supreme Court case of 
Florida State v. Jardines [77]. As a result, the acceptance of canine discoveries as evidence in courts will only continue if its credibility, accuracy, and reliability of canine training are maintained. To maintain these standards, the Scientific Working Group on Dog \& Orthogonal detector Guidelines (SWGDOG) created in 2004 to provide recommended parameters for optimal, accurate, and reliable canine detection performance and acceptance in the court of law. However, SWG's have been disbanded by the federal government and the work of SWGDOG will continue through the establishment of the Organization of Scientific Area Committees (OSAC).

\section{INSTRUMENTAL APPROACHES}

The analytical approach utilized in this research consisted of the headspace evaluation of the collected explosive odor using solid phase micro-extraction (SPME) followed by analysis using both gas chromatography-mass spectrometry (GC/MS) and gas chromatography-electron capture detection (GC/ECD) in order to identify the volatile chemical profile of each explosive odor class at room temperature.

\subsection{Solid Phase Microextraction (SPME)}

Solid phase microextraction (SPME) is a solventless, simple, fast, and efficient sample preparation technique developed by Prof. Janusz Pawliszyn in 1989 [78]. This technique utilizes a short, thin, solid rod of fused silica (typically $1 \mathrm{~cm}$ long and $0.11 \mathrm{~mm}$ outer diameter), coated with an absorbent polymer (fiber) which is attached to a metal rod and mounted on a syringe-like device for extraction of analytes. The fiber allows for chemical analysis without sample disturbance while pre- concentrating the volatile 
compounds. During sampling, the metal rode of the SPME fiber is inserted into the vial containing the sample and by pressing down the plunger the fiber is exposed for a certain time to extract analytes from the sample matrix [79] ( as seen in Figure 2).

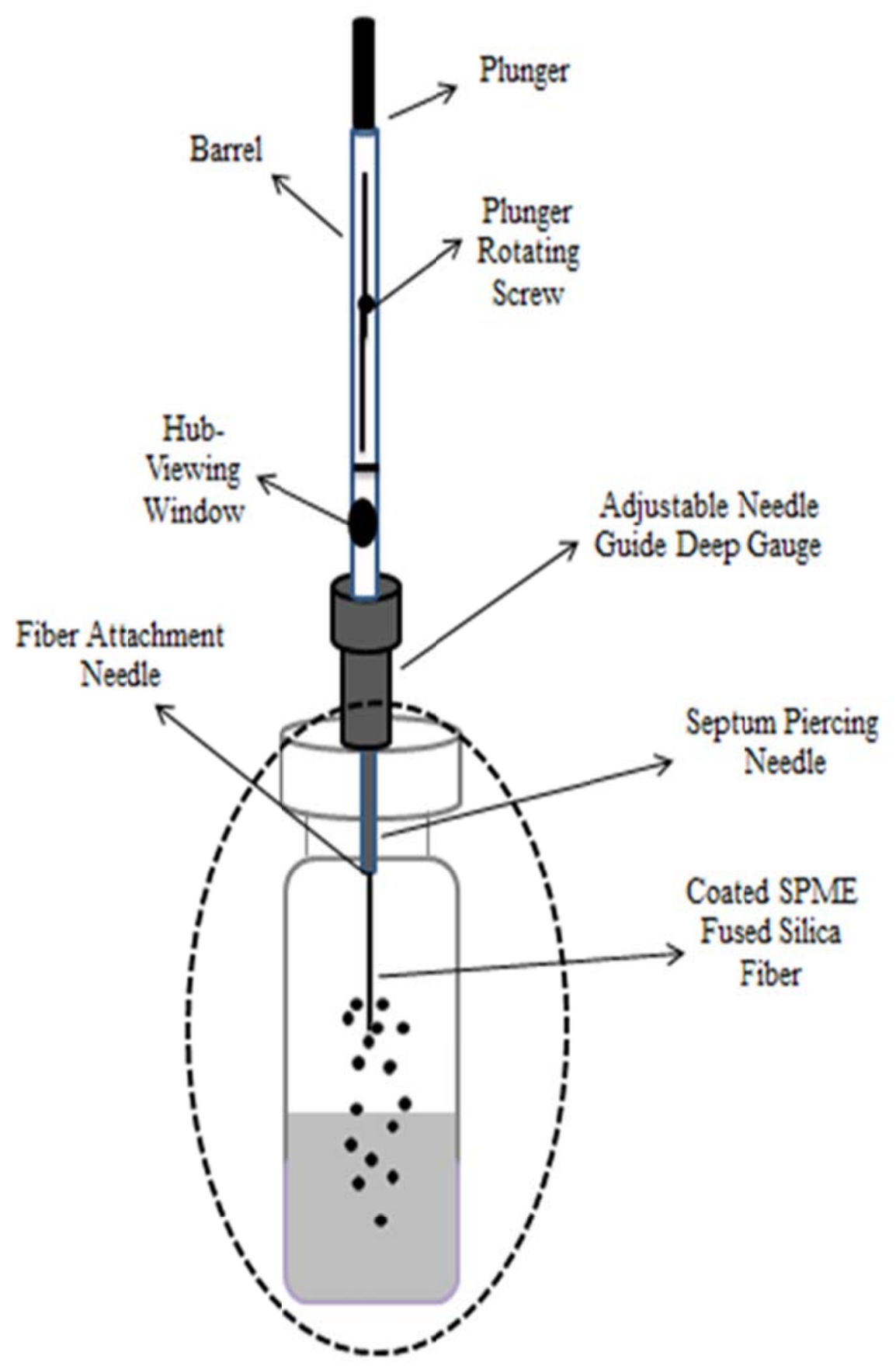

Figure 2: Solid Phase Microextraction Device (80) 
Extraction can be performed either by direct immersion though a liquid sample or by exposing the fiber to the headspace of a sample. The extraction process involves the absorption of the analytes onto the polymer coating of the fiber. Those extracted analytes eventually will be thermally desorbed by injecting the fiber into a gas chromatograph (GC) or liquid chromatograph (LC) for both qualitative and quantitative analysis [79].

In SPME, the extraction is considered to be complete when it reaches equilibrium. The equilibrium is achieved among the concentrations of the analyte in the headspace above the sample, and in the polymer coating on the fused silica fiber. These conditions can be described by the following equation:

\section{Equation 1}

$$
C_{0} V_{s}=C_{s}^{\infty} V_{s}+C_{f}^{\infty} V_{f}
$$

Where $C_{o}$ is the initial concentration of analyte in the sample, $V_{s}$ : volume of sample, $V_{f}$ :

volume of fiber coating, $\mathrm{C}_{\mathrm{f}}^{\infty}$ : equilibrium concentration on the fiber, $\mathrm{C}_{\mathrm{s}}^{\infty}$ : equilibrium concentration in the sample. The analyte adsorbed by the fiber depends on the thickness of the polymer coating and on the distribution constant $\left(\mathrm{K}_{\mathrm{fs}}\right)$ of the analyte between the sample and the coating fiber (expressed in Equation 2). The distribution constant generally increases with increasing molecular weight and boiling point of the analyte $[79,80]$.

\section{Equation 2}

$$
K_{f s}=C_{f}^{\infty} V_{f}+C_{s}^{\infty} V_{s}
$$

Quantitation of the number of moles (n) extracted from the analyte by the fiber coating can be described in Equation 3 (which combines Equations 1 and 2). The Equation 
clearly indicates the linear relationship between the analytes present within the fiber coating and the initial concentration of the analytes in the sample $[79,80]$.

\section{Equation 3}

$$
\boldsymbol{n}=C_{f}^{\infty} V_{f}=\frac{K_{f s} V_{f} V_{s} C_{0}}{K_{f s} V_{f}+V_{s}}
$$

For a three-phase system (in which headspace is included), Equation 4 applies for equilibrium conditions. The $\mathrm{K}_{\mathrm{hs}}$ represents the partition coefficient of the analyte between the headspace and the sample matrix.

\section{Equation 4}

$$
\boldsymbol{n}=C_{f}^{\infty} V_{f}=\frac{K_{f s} V_{f} V_{s} C_{0}}{K_{f s} V_{f}+K_{h s} V_{h}+V_{s}}
$$

This equation (4) states that the amount of analyte extracted is independent of the location of the fiber in the sample (in the headspace or directly in the sample), as long as the volumes of the fiber coating, headspace, and sample matrix remain constant $[79,80]$.

There are two types of SPME fibers: absorbent and adsorbent. An absorbent fiber acts like sponge in which the analyte migrate freely through the coating and the ability of the coating to efficiently retain the analyte depends merely on the size of the analyte itself and in the thickness of the coating. Polarity of the fiber may contribute to attract analyte but it is the thickness of the coating which allows the analyte to migrate in and out without competition between analytes. Adsorbent type fibers are usually solids with high surface areas or internal pores. These fibers physically interact with the analytes (which are trapped within the porous material) through hydrogen bonding or Van der Waals 
interactions. Meaning that analytes can compete because there are a limited number of pores sites. Therefore, this can result in a reduction or displacement of analytes with low affinity by those with higher affinity for a pore site [81].

Table 1: Summary of Fiber Coatings Available for SPME Fibers

\begin{tabular}{cccc}
\hline Fiber coating & Fiber Type & $\begin{array}{c}\text { Film } \\
\text { Thickness } \\
(\boldsymbol{\mu m})\end{array}$ & $\begin{array}{c}\text { Recommended Applications } \\
\text { and Molecular Weight (MW) } \\
\text { Ranges (amu) }\end{array}$ \\
\hline $\begin{array}{c}\text { Polydimethylsiloxane } \\
\text { (PDMS) }\end{array}$ & Absorption & $100,30,7$ & $\begin{array}{c}\text { Non-polar volatiles (MW 80- } \\
\text { 300) }\end{array}$ \\
$\begin{array}{c}\text { Polyacrylate } \\
\text { (PA) }\end{array}$ & Absorption & 85 & $\begin{array}{c}\text { Polar semi-volatiles } \\
\text { (MW 80-300) }\end{array}$ \\
$\begin{array}{c}\text { Carboxen- } \\
\text { polydimethylsiloxane } \\
\text { (CAR-PDMS) }\end{array}$ & Adsorption & 75 & $\begin{array}{c}\text { Gases and low molecular weight } \\
\text { compounds } \\
\text { (MW 30-225) }\end{array}$ \\
$\begin{array}{c}\text { Divinylbenzene/Carboxen/ } / \text { (M) } \\
\text { Polydimethylsiloxane } \\
\text { (DVB/CAR/PDMS)) }\end{array}$ & Adsorption & 50,30 & $\begin{array}{c}\text { Alcohols and polar compounds } \\
\text { C3-C20; MW 40-275 }\end{array}$ \\
$\begin{array}{c}\text { Polydimethylsiloxane- } \\
\text { divenylbenzene } \\
\text { (PDMS-DVB) }\end{array}$ & Adsorption & 60,65 & $\begin{array}{c}\text { Volatiles, amines, and nitro- } \\
\text { aromatic compounds (MW 50- } \\
\text { 300) }\end{array}$ \\
\hline
\end{tabular}

A variety of polymers are available for SPME fiber coatings. For example: Polydimethylsiloxane (PDMS) which is apolar and has a high affinity for non-polar compounds and polyacrylate (PA) fiber coatings which is more polar and therefore extract polar compounds. Fiber coatings containing porous and adsorbent materials include mixtures of divinylbenzene (DVB) or Carbowax (CW) with PDMS [78]. 
Selectivity can be altered by changing the type of polymer coating on the fiber, or the coating thickness. In general, volatile compounds require a thick coating, and a thin coating is most effective for adsorbing/desorbing semivolatile analytes. The use of a thicker fiber requires a long extraction time and usually recoveries are higher. Polar fibers are used for polar analytes and non-polar fibers for non-polar analytes [78]. Solid Phase Microextraction (SPME) offers a wide variety of fibers that can be utilized according to the sample matrix under study. Table 1 shows the different fiber coatings available for SPME fibers $[78,82]$.

Solid Phase Microextraction (SPME) offers many advantages such as being a non-destructive technique since it uses only a small portion of the sample, also fibers are reusable (100+ times), cost efficient, and useful for analysis of volatiles, semi-volatiles and non-volatiles in gases, liquids, and solids. Solid Phase Microextraction (SPME) incorporates sampling, extraction, pre-concentration, and sample introduction in just one step which makes it a very fast sample analysis technique $[78,80]$. In addition, it provides high sensitivity and can be coupled with other instruments besides GC like CE, LC, MALDI, and MS [78].

Solid Phase Microextraction (SPME) has a wide range of applications, just to name a few, SPME has been utilized in environmental analysis [83] for the studies of pesticides, herbicides, and other biologically active compounds in aqueous samples [84,85] and analysis of polycyclic aromatic hydrocarbons (PAHs) [86]. Other applications of SPME includes food chemistry for the qualitative and quantitative analyses of volatile organic compounds in wine, candies and herbs [87], for the analysis 
of common flavors in coffee and some fruit beverages [88], for analysis of milk, cheese, and whey powder [89]. For the characterization of different alcoholic drinks [90,91,92]. In other areas such drug analysis and toxicology, Solid Phase Microextraction (SPME) has been utilized for example to describe the presence of cannabis in hair [93], as well as analysis of blood, urine, saliva, and oral fluids [94]. In addition, SPME was applied for the analysis of amphetamines, cocaine and metabolites, cannabinoids, methadone, other opioids and various other therapeutic drugs [95]. Solid Phase Microextraction (SPME) has also been useful in criminal investigations and forensic analysis. For example, the analysis of ignitable liquid residues present on the skin of arson suspects [96] and for explosive trace recovery $[97,98]$.

\subsection{Gas Chromatography (GC)}

Gas Chromatography (GC) is a technique used to separate volatile components of a mixture. It requires a mobile and a stationary phase [99]. The mobile phase is the carrier gas such as helium, nitrogen, or hydrogen and the stationary phase is the column. In GC a syringe needle is placed into a hot injector port at a temperature higher than the boiling points of the volatiles present in the sample. Once the sample is injected into the GC it becomes vaporized, then the mobile phase will move it into the column separating the sample into its components. There are three different types of injection modes: split, splitless, and on column modes. In the split injection mode, the split exit vent is open and when evaporation occurs, only a small fraction of the gas can be introduced into the column. The purpose of this type of injection is to reduce the amount of sample entering the column. For this reason split injection is not suitable for trace analysis. In splitless 
injection mode, the split exit remains closed. In this way, when the sample is introduced into the injector port where it rapidly evaporates is transferred into the column by means of the column flow. Splitless injection is ideal for trace analysis since the entire sample is introduced on to the column. For this reason, this research utilized splitless injection for analysis of explosive volatiles. In the on-column injection mode, the sample is introduced directly into the unheated inlet of the capillary column without being evaporated. The entire sample is introduced into the column. This type of injection mode is the less commonly used. During chromatographic analysis, the components that are not held by the stationary phase move quickly through the column and the components held tightly by the stationary phase move slowly at different times (:retention time). A detector is then used to identify the components of the mixture according to the order in which they are eluted off the chromatographic column $[99,100]$.

\subsubsection{Detectors}

There are many types of detectors used in gas chromatography. However, detectors such as mass spectrometry (MS) and electron capture detector (ECD) are the most commonly used for explosive analysis since these detectors possess some advantages such as identification capability by MS and the high sensitivity of the ECD. For the purpose of this research, these two detectors will be utilized for analysis of explosive volatiles [99]. 


\subsubsection{Mass Spectrometry (MS)}

In mass spectrometry, once the injected sample is vaporized the molecules need to become charged in the gas-phase prior to entrance to the mass spectrometer. Ionization takes place in the ion source where an electromagnetic field causes molecules of the sample to be blasted with electrons, which cause them to break into pieces and turn into charged particles called ions. These ions are filtered by the quadrupole component and eventually the detector in the MS will record the abundance of these ions based on their mass to charge ratios $(\mathrm{m} / \mathrm{z})$. The separated ions are then measured, and the results displayed on a mass spectrum chart. Therefore, mass spectrometry allows identification of atoms or molecules and provides structural elucidation of distinctive fragmentation patterns [99].

\subsubsection{Electron Capture Detector (ECD)}

In an electron capture detector (ECD), a radioactive source of electrons (usually $\mathrm{Ni}^{63}$ ) ionizes the carrier gas by emitting electrons (beta particles). The electrons emitted collide with the molecules of the carrier gas, resulting in many more free electrons. The burst of electrons decreases in the presence of organic molecules containing electronegative compounds such as chlorinated, fluorinated, or brominated molecules which tend to capture electrons. Electron capture detector (ECD) is highly selective, sensitive, produces a fast response, and has the advantage of not altering the sample because only a minimal amount of sample is require [99]. 


\section{RESEARCH OBJECTIVES}

The main objectives of this research included laboratory experiments designed to optimize operational parameters of the HSCS for the creation of training aids which followed a previous evaluation of the signature odor chemicals of four explosive families: 2,3-Dimethyl-dinitrobutane (DMNB) representing the nitro-alkanes, 2-ethyl-1-hexanol (P) representing the plasticizer commonly found in conjunction with trinitrotriazacyclohexane (RDX) and 1,3,5,7-Tetranitro-1,3,5,7-tetrazacyclooctane (HMX) representing the nitro amines, 2,4-dinitritoluene (2,4-DNT) representing the nitroaromatics, and nitroglycerin (NG) representing the nitrate-esters. All samples were collected onto a gauze pad using the HSCS as the method of collection and the gauze pads were then subjected to SPME-GC-MS/ECD analysis to identify collected volatiles. This study also focused on field testing with certified canine teams to evaluate their capabilities to detect explosive odorants collected from real explosive material via the dynamic airflow system. Tasks presented in this study are described below:

I. Evaluation of signature volatiles from explosive families

II. Evaluation and optimization of the flow rates (low, medium, and high) at 30 seconds of HSCS using representative VOCs

III. Evaluation and optimization of sampling time ( $30 \mathrm{sec}$ vs. $60 \mathrm{sec}$ ) of representative VOC accumulation using HSCS

IV. Comparison of static vs. dynamic collection of representative VOCs

V. Evaluation of longevity of sorbent materials containing the extracted VOCs and the selection of an optimal storage containment system 
VI. Field evaluation of laboratory HSCS training aids with optimized parameters using certified explosive detection canines

\section{METHODOLOGY}

\subsection{Materials}

\subsubsection{Explosive materials}

Samples utilized for analysis of C4 explosive material were obtained from City of Miami Police, Metro Dade K9 facility, and The Bureau of Alcohol, Tobacco, Firearms, and Explosives (ATF) in Tennessee. Trinitrotoluene (TNT) was also obtained from the ATF in Tennessee. Single based smokeless powder \# 4896 was obtained from Hodgdon Powder Company. Double based smokeless powder Accurate \#7 was obtained from Cabela's. For field evaluations, explosive samples were provided by the United States Marine Corps at Camp Pendleton, California.

\subsubsection{Sorbent Materials}

Odorants were collected onto 4" x 4" absorbent gauze material from DUKAL Corporation obtained from Ronkonkoma, NY, USA. Pre-treatment of the collection material was performed when necessary and consisted of a direct spike with four milliliters of HPLC grade methanol (Fisher Scientific, Pittsburgh, PA, USA) followed by heating in the oven at a temperature of $105{ }^{\circ} \mathrm{C}$ for one hour. The purpose of the pretreatment was to eliminate any remnants of possible VOCs present within the gauze pad which can cause any interference with the detection of the target compound and to decrease background signal. 


\subsubsection{Odor Collection Materials}

Eight (8) ounces mason crystal jars were obtained from Publix supermarket and utilized for collection of explosive material. The vials utilized to hold the collected samples were 40-ml glass, clear, screw top vials with PTFE/Silicone septa (SUPELCO, Bellefonte, PA, USA). Alcohol pads utilized for cleaning the equipment were 1.1" x 2.6" PDI (Professional Disposables International, Inc.) obtained from Orangeburg, NY, USA.

\subsubsection{Laboratory Supplies}

The Solid Phase Micro-Extraction (SPME) fibers utilized to analyze the headspace of all samples were 60 um polydimethylsiloxane (PDMS) (blue) obtained from Supelco (Bellefonte, PA). The specific fiber type was chosen because it is designed to collect high polar volatiles and semi-volatiles at a trace level. The fibers where conditioned prior to use for $30 \mathrm{~min}$ at $250^{\circ} \mathrm{C}$ according to manufacturer recommendations. The fiber was inserted through the septum and exposed approximately $1.0 \mathrm{~cm}$ above the sample within the optimized time prior to $\mathrm{GC}$ analysis.

Chemical standards used for external calibration included 2-ethyl-1-hexanol (> 99.6\%) and 2, 3-dimethyl-2,3-dinitrobutane ( $98 \%$ ), both obtained from Sigma Aldrich (St Louis, MO, USA). 2, 4- dinitrotoluene and nitroglycerin standards $(1000 \mu \mathrm{g} / \mathrm{mL}$ in $\mathrm{MeOH}$ ampule) were obtained from Accustandard (New haven, $\mathrm{Ct}$ ).

The storage containment systems tested were: $40 \mathrm{ml}$ clear glass screw top vials with PTFESilicone septa, aluminum bags (heat sealed) 6" x 5.5" and ziploc bags (double zipper) 6.5" x 5.875" obtained from Supelco, TED-Pella INC, and Publix supermarket respectively. 


\subsection{Statistical Data Analysis}

When a study is conducted and the dependent variable is measured, a set of numbers is obtained. Those numbers inevitably are not the same; this is the result of factors such as individual differences or experimental error. The objective is to obtain from those numbers a meaningful conclusion regarding the influence of the independent variables. Statistical data analysis provides a process to evaluate any information with a view to reach to a certain meaningful conclusion for a given situation. Statistical data analysis can be done by different methods as according to the needs and requirements of the study. To determine if a result is statistically significant, ANOVA or Analysis of Variance is utilized as a significance test in the evaluation of experimental results. The analysis of the data generated in this work was analyzed by ANOVA.

ANOVA is a statistical method used to determine the existence of statistically significant difference between two or more means. This test uses variances (square of the standard deviation) to determine if means subject to analysis are different or not. In ANOVA if there is only one factor (or dependent variable) it is called a one-way ANOVA. But if two factors are present, then it is called a two-way ANOVA [101]. For the purposes of this research, one-way ANOVA was applied to compare the results obtained from multiple samplings of the same operational parameter (i.e. airflow, time) and observe if there was a significant difference among the amount of target odor signature collected.

Analysis of variance (ANOVA) involves the partitioning of variance of the dependent variable into different components: between groups and within groups' variability. The between groups term is calculated by comparing the mean of each group with the overall 
mean of the data. Within groups term refers to the variation of each observation from its group mean.

The test for ANOVA is the ANOVA F-test (named for R. Fisher who has developed this test in 1920). This test is the ratio of the average variability between groups to the average variability within groups. Therefore, F-test tells how big a difference is between the given conditions. If the average difference between groups is similar to that within groups, the F ratio is about 1. As the average difference between groups becomes greater than that within groups, the F ratio becomes larger than 1 . Then when the calculated $\mathrm{F}$ value is greater than the $\mathrm{F}$ critical value variances are significant different. As an alternative to using the $\mathrm{F}$ values, ANOVA estimates the $\mathrm{P}$ value to indicate the degree of confidence we have that there is a significant difference between means. The critical p-value is set at 0.05 . Any $p$-value that is lower than 0.05 results in a statistically significant result, while any p-value above 0.05 does not present any statistically significant evidence [101,102]. Larger F-ratios gives smaller P-values.

In general, ANOVA represents a flexible way for data analysis. It provides information based on the partitioning of variance. It also provides necessary information for decision making through the use of a statistical test and helps to determine which factor has more impact on the response. Analysis of variance (ANOVA) can be performed by using specialized statistical software package or excel to a wide range of applications in analytical work. Minitab 14 Statistical Software was utilized to analyze all data collected in this research.

In addition, to evaluate canine's performance, the positive predictive value ( $P P V)$ and the negative predictive value $(N P V)$ statistical analysis was conducted. 
The positive predicted value is defined as $P P V$ where a "true positive" refers to the event that the test makes a positive prediction, and the canine gives a positive response. And the "false positive" is the event that the test makes a positive prediction, and the canine has a negative response.

$$
P P V=\frac{\text { True Positive }}{\text { True Positive }+ \text { False Positive }}
$$

The negative predicted value is defined as $N P V$ where a "true negative" refers to the event that the test makes a negative prediction, and the canine has a negative response. And a "false negative" is the event that the test makes a negative prediction, and the canine has a positive response.

$$
N P V=\frac{\text { True Negative }}{\text { True Negative }+ \text { False Negative }}
$$

\subsection{HSCS Device for Collection of Explosive Volatiles}

The HSCS developed by Battelle Memorial Institute is a dynamic airflow device used for the collection of VOCs from various biological specimens that can withstand the rigors of field operations. The Human scent collection system was developed after its counterpart the Scent Transfer Unit (STU-100). This device has a lightweight body, a user-friendly interface, an internal power supply, and a digital system that provides a battery life indicator, settings for times selection modes of 30 and 60 seconds, and 3 distinctive flow rates: low, medium, and high for actual scent collection purposes. 


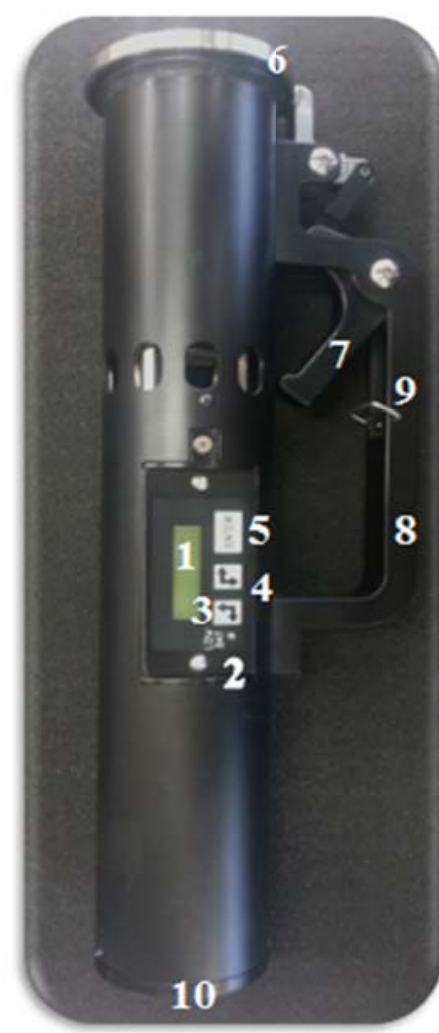

1. Display screen

2. Low battery indicator light

3. Up arrow button

4. Down arrow button

5. ENTER button

6. Gauze pad holder

7. Gauze pad release

8. Handle

9. Locking clasp

10. Battery cover

Figure 3: Human Scent Collection System (HSCS)

The design of the HSCS allows cleaning of the device with alcohol pads on the interior and the exterior between sample analyses to minimize cross contamination. The diagram of the Human Scent Collection system (HSCS) is depicted in Figure 3.

The dynamic airflow methodology allows for the collection of multiple samples from the same source, preventing damage to other trace evidence items such as fingerprints, blood, etc. while providing forensic analysts the ability to collect valuable evidence from the field. Its potential value has been demonstrated as observed in experiments collecting human scent from improvised explosive devices to trace individuals who handled it 
[103]. In view of its capability for collecting trace amounts of evidence, this research utilized the HSCS as a novel and viable way of collecting newly emerging explosive odorants in combat areas for future development of canine training aids.

\subsubsection{HSCS Airflow Velocity}

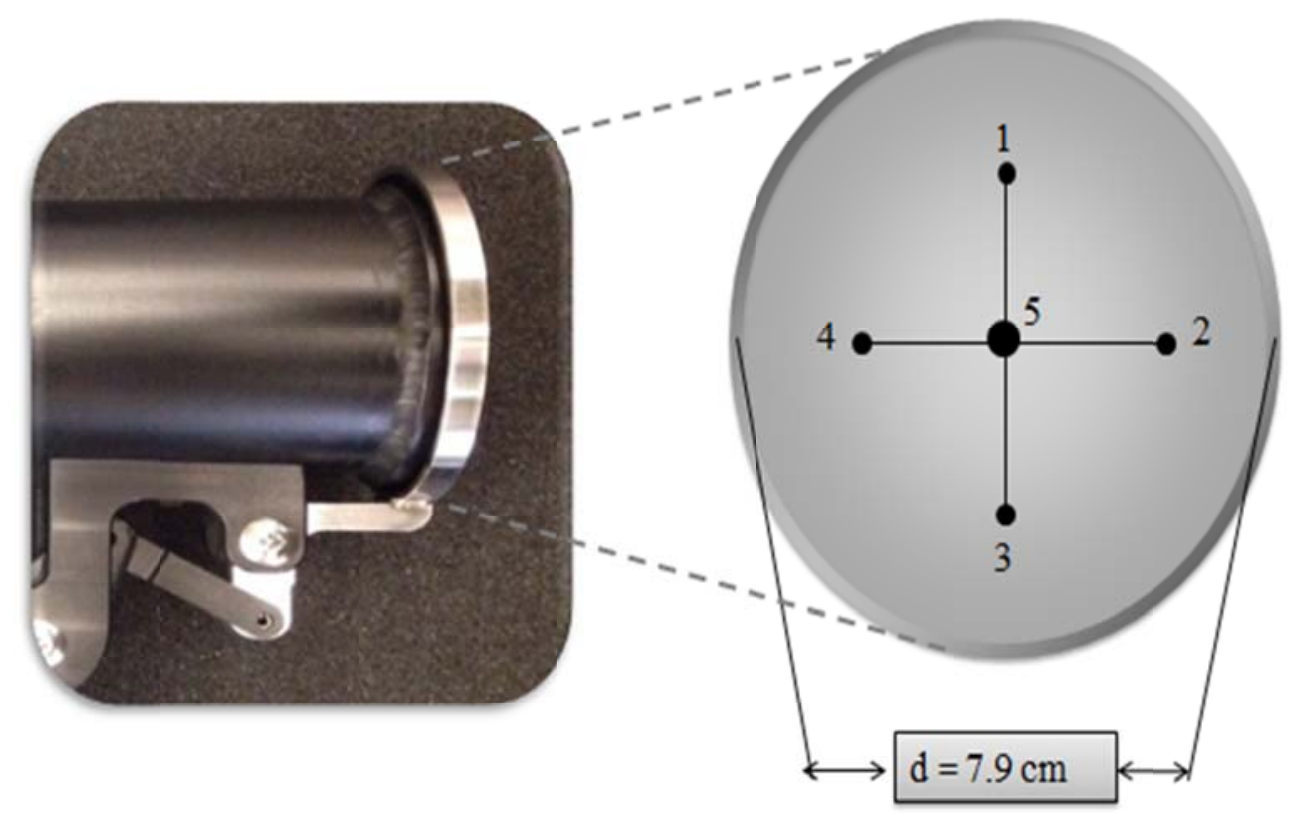

Figure 4: Linear Traverse for Round Duct $7.9 \mathrm{~cm}$-Diameter Approach

As an initial step for the HSCS optimization, a preliminary evaluation of the air flow velocity and capacity of the HSCS device was performed. In order to do this, measurements of the airflow velocity at each available speed was closely monitored. This was performed by conducting airflow measurements at five (5) different locations on the surface of the HSCS opening as can be observed in Figure 4. 
The task required to perform a traverse of the opening to measure the velocity at the selected five (5) points. Upon calculation of the mean velocity, the value is multiplied by the cross-sectional area of the opening to obtain the total volumetric flow rate measurement in cubic feet per minute (CFM) [104].

Table 2 Calculations for the HSCS Air Flow Velocity Measurements

\begin{tabular}{cc}
\hline Conversions & Diameter of the duct $=\mathbf{7 . 9} \mathbf{~ c m}$ \\
\hline $\mathbf{1} \mathbf{c m}^{2}=\mathbf{0 . 0 0 1 0 7 6} \mathbf{f t}^{2}$ & $\mathrm{~A}=\Pi^{2}$ \\
$\mathbf{1} \mathbf{m p h}=\mathbf{8 8 f t} / \mathbf{m i n}$ & $\mathrm{A}=3.1416^{*}(3.95 \mathrm{~cm})^{2}$ \\
$\mathbf{1 C F M}=\mathbf{0 . 4 7 1 9 5} \mathbf{L} / \mathbf{s e c}$ & $\mathrm{A}=49.02 \mathrm{~cm}^{2}$ \\
& $\mathrm{~A}=0.0527 \mathrm{ft}^{2}$ \\
\hline
\end{tabular}

An anemometer is an instrument commonly used to measure air velocity, air volume, and temperature. Many types of anemometers are manufactured but for the purpose of this experiment, a rotating vane anemometer was utilized to take air flow measurements The anemometer was placed directly below the circular opening (with a diameter of 7.9 $\mathrm{cm}$ ) of the HSCS to monitor the airflow volume passing through in the downward position ( as seen in Figure 5), where nine (9) consecutive readings were taken at each of the selected points with and without collection medium (4"x 4" cotton gauze pad) at $90^{\circ}$ apart including the center for the three air flow rates available: low, medium, and high. Measurements at the established five locations were given in miles per hour and when the mean velocity at each location was found, all the values were added together to get a final value which was converted to $\mathrm{ft} / \mathrm{min}$. The value was then multiplied by the area (A) of the circular opening (in ft2). Proper conversions were applied in order to obtain volumetric results in $\mathrm{L} / \mathrm{min}$, the mean velocities (mph) for the three flow rates are 
found on Table 2. Measurements were taken at indoor laboratory conditions $\left(22.6^{\circ} \mathrm{C}\right.$ and $56.5 \%$ relative humidity).

According to the results, when sampling in a downward position the HSCS produces an airflow volume greater without any material but when the gauze pad is loaded into position the airflow volume drops significantly. For example; at the highest speed of the device, the HSCS reports an airflow volume of $486 \mathrm{~L} / \mathrm{min}$ without any absorbent material compared with $368 \mathrm{~L} / \mathrm{min}$ when the gauze pad is loaded. Figure 6 and Table 3 present a summary of the airflow volume measurements at all available speeds of the device.

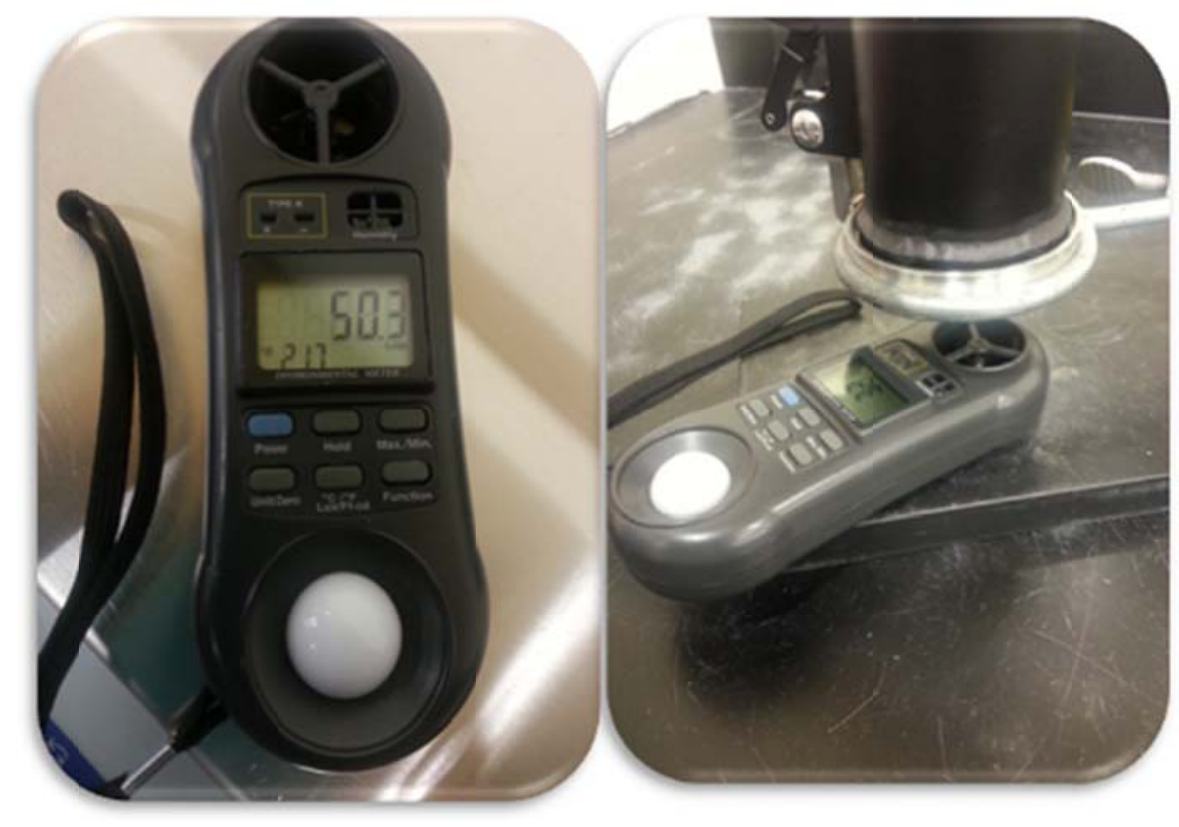

Figure 5: HSCS Air Flow Velocity Measurements Taken by an Anemometer 


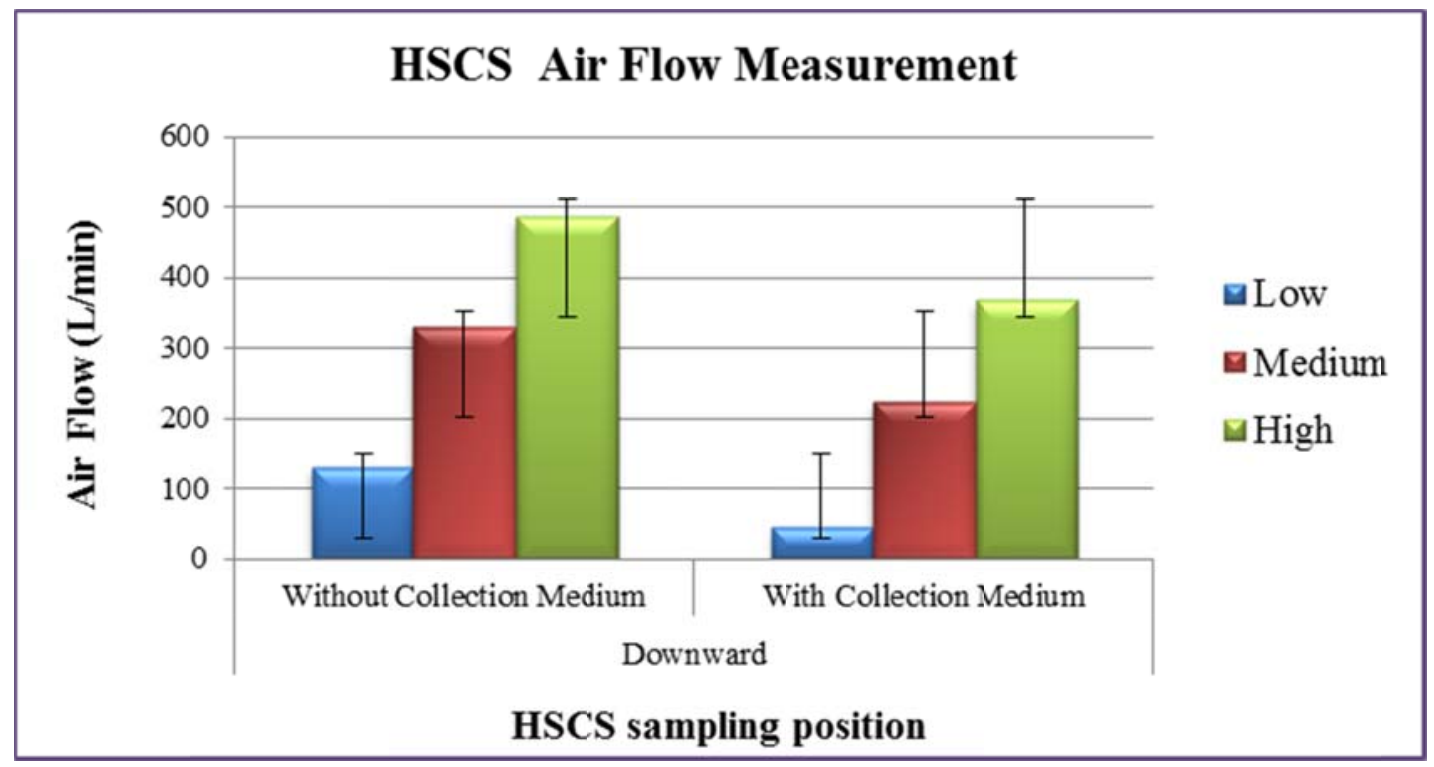

Figure 6: HSCS Air Flow Volume at all Speeds in Liter/Minutes

Table 3. Summary of the Air Flow Volume Measurements

\begin{tabular}{|l|c|c|}
\multicolumn{1}{c}{} & \multicolumn{1}{c}{$\begin{array}{c}\text { Without Collection } \\
\text { Medium (L/min) }\end{array}$} & $\begin{array}{c}\text { With Collection } \\
\text { Medium (L/min) }\end{array}$ \\
\hline Low $\square$ & $131.3 \pm 0.005$ & $45.96 \pm 0.004$ \\
\hline Medium $\square$ & $329.6 \pm 0.191$ & $223.2 \pm 0.015$ \\
\hline High $=$ & $485.9 \pm 0.082$ & $367.7 \pm 0.071$ \\
\hline
\end{tabular}

\subsubsection{HSCS Protocol for Collection of Explosive Volatiles}

For HSCS collection of explosive volatiles, eight (8) ounces glass jars were selected because they perfectly fit the opening site of the HSCS device and allow the isolation of explosive material for dynamic collection. The protocol for dynamic collection involved placing the HSCS approximately 2 to 3 inches from the top of the glass container and holding in place in a downward position using ring stands (Figure 7). The collection 
material was a 4" $\mathrm{x} 4$ " sterile cotton gauze pad and the samples were stored in silanized $40 \mathrm{ml}$ glass vials after collection was completed. Temperature and relative humidity during sampling was recorded by the use of an anemometer. In addition, the HSCS device was cleaned with alcohol pads before first use and every time a sample was taken. Proper precautions were followed in order to prevent cross contamination of sampling material.
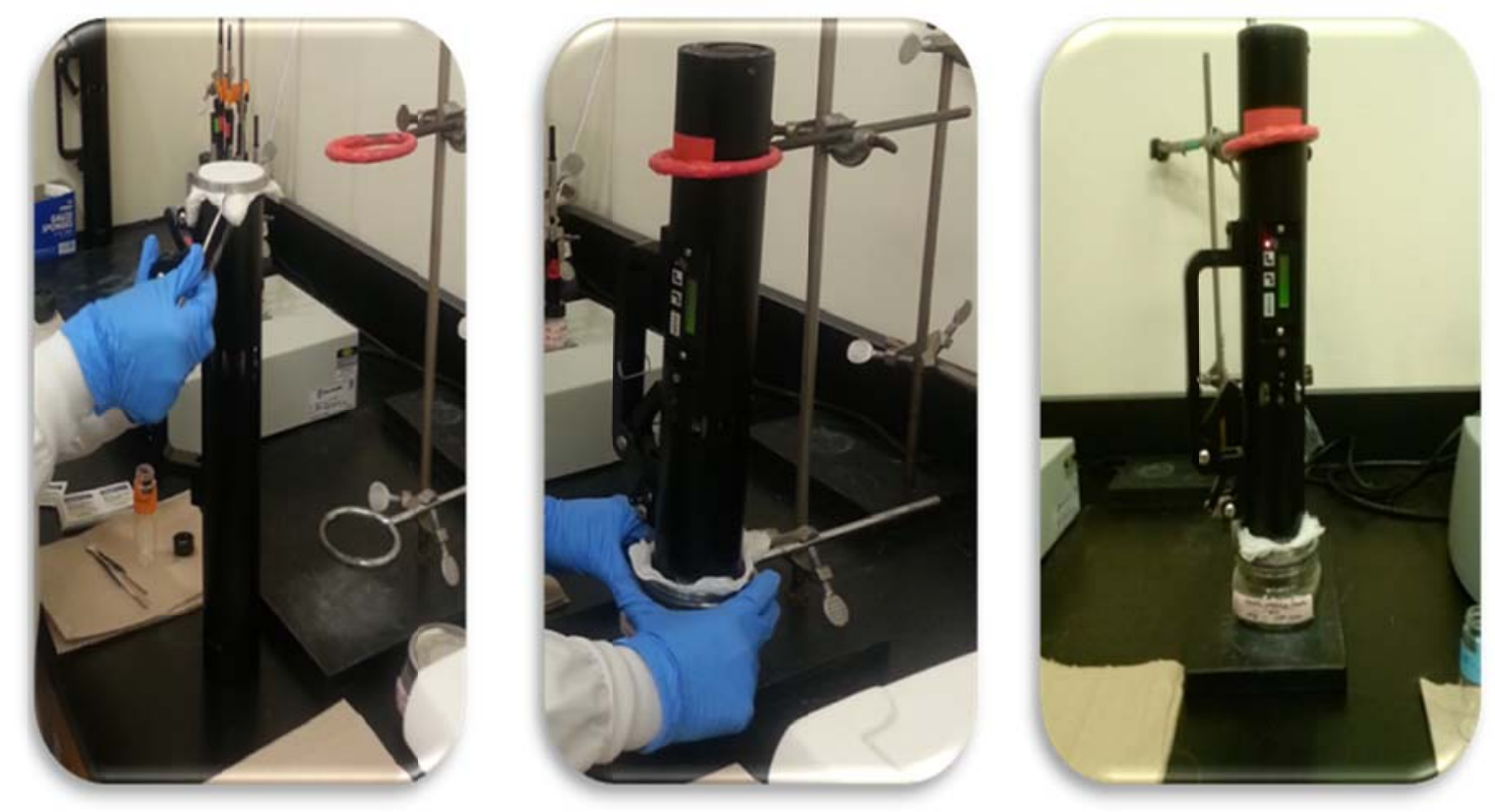

Figure 7: HSCS System Setup for Explosive VOCs Collection

\subsection{GC/MS Method for Analysis of 2E1H and DMNB Representative VOCs}

The GC/MS method employed for the detection of 2E1H and DMNB odorants used the Agilent 6890-5973 combination running Chemstation software. A GC-MS method starting at $40^{\circ} \mathrm{C}$ was programmed to hold for $4 \mathrm{~min}$, then heating at $10^{\circ} \mathrm{C} / \mathrm{min}$ up to 300 
${ }^{\circ} \mathrm{C}$ and held for $2 \mathrm{~min}$ for a total run time of $32 \mathrm{~min}$. Helium was the carrier gas at a flow rate of $1 \mathrm{~mL} / \mathrm{min}$ at an average velocity of $37 \mathrm{~cm} / \mathrm{sec}$. The column used was an HP5 30 $\mathrm{m}, 0.25 \mathrm{~mm}$ i.d, $25 \mathrm{um}$ film thickness column. The injection port was held at $265^{\circ} \mathrm{C}$ with a pressure of $7.00 \mathrm{psi}$ and 5 min SPME desorption time. The analysis was conducted under splitless mode and the MS was operated in electron ionization (EI) full scan mode from 45 to $500 \mathrm{amu}$, with a 4 min solvent delay.

\subsection{GC/ECD Method for Analysis of 2, 4 DNT and NG Representative VOCs}

\section{2, 4 Dinitrotoluene}

Representative odorant 2, 4-DNT from single based smokeless powder was determined through SPME, which was combined with gas chromatography electron capture detector to produce a comprehensive screening method that was optimized for the detection of the desired explosive volatile. The GC used was an Agilent 6890-5973 combination running Chemstation software with ECD and the column employed was a 6.0m 0.53mm i.d., 1.5 um film thickness Restek (Bellefonte, PA) Rtx ${ }^{\circledR}-\mathrm{TNT}$ for explosives analysis. The injection port was held at $265{ }^{\circ} \mathrm{C}$ with a 5 min SPME desorption. The oven program was set initially for $2 \mathrm{~min}$ at $80^{\circ} \mathrm{C}$ followed by $25^{\circ} \mathrm{C} / \mathrm{min}$ ramp to $300{ }^{\circ} \mathrm{C}$ with a $5 \mathrm{~min}$ hold for a total run of $15.80 \mathrm{~min}$. Analysis was conducted under splitless mode with a solvent delay of $0.5 \mathrm{~min}$. The detector was held at $320{ }^{\circ} \mathrm{C}$ with anode purge and the carrier was Helium at $15 \mathrm{~mL} / \mathrm{min}$ with a Nitrogen $60 \mathrm{~mL} / \mathrm{min}$ makeup in the ECD. 


\section{Nitroglycerin (NG)}

The optimized method for the detection of NG representative odorant from double based smokeless powder was also determined through SPME/GC-ECD. The same GC instrument and column employed for analysis of 2,4 DNT was utilized as explained above. The injection port was held at $230{ }^{\circ} \mathrm{C}$ with a 5 min SPME desorption. The oven program was set initially at $40{ }^{\circ} \mathrm{C}$ followed by $10{ }^{\circ} \mathrm{C} / \mathrm{min}$ ramp to $150{ }^{\circ} \mathrm{C}$ and then 18 ${ }^{\circ} \mathrm{C} / \mathrm{min}$ ramp to $250{ }^{\circ} \mathrm{C}$ with a $5 \mathrm{~min}$ hold for a total run of $21.56 \mathrm{~min}$. Analysis was conducted under splitless mode with a solvent delay of $0.5 \mathrm{~min}$. The detector was held at $260{ }^{\circ} \mathrm{C}$ with anode purge and the carrier was Helium at $17 \mathrm{~mL} / \mathrm{min}$ with a Nitrogen 60 $\mathrm{mL} / \mathrm{min}$ makeup in the ECD.

\subsection{SPME Analysis and Extraction Procedures}

- Evaluation of Signature Volatiles from Explosive Families

\section{2-ethyl-1-hexanol (2E1H) and 2, 3-Dimethyl-dinitrobutane (DMNB)}

Solid phase microextraction (SPME) analysis of signature volatiles present in the headspace of the $\mathrm{C} 4$ tagged explosive (Composition 4) was performed under atmospheric conditions followed by thermal desorption into a GC/MS.

Samples of $\mathrm{C} 4$ explosive material were obtained from three different sources: City of Miami Police, Metro Dade K9 facility, and the Bureau of Alcohol, Tobacco, Firearms, and Explosives (ATF) in Tennessee which were classified as source \#1, source \#2, and source \# 3 respectively. 
From source \#1, \#2, and \# 3, three samples of $C 4$ explosive material with a mass average of $157 \mathrm{~g}$ were placed each in individual glass containers of $16 \mathrm{oz}, 284 \mathrm{~g}$ placed in glass containers of $503 / 4 \mathrm{oz}$, and $70 \mathrm{~g}$ placed in $8 \mathrm{oz}$ glass containers respectively. All samples were given one (1) hr to equilibrate followed by one (1) hr SPME extraction in order to confirm the presence of $2 \mathrm{E} 1 \mathrm{H}$ and DMNB signature odorants. Triplicate samples were taken along with a correspondent blank to control for any cross contamination and as a control for proper instrument detection.

\section{2, 4- Dinitrotoluene (2, 4 -DNT)}

Single based smokeless powder and TNT were used to confirm the presence of 2,4-dinitrotoluene $(2,4-\mathrm{DNT})$ odorant representative from the nitro aromatic group. For the analysis of single based smokeless powder performed in the laboratory, a preliminary evaluation was performed in which three (3) crystal jars (8 oz) containing a mass average of $25 \mathrm{~g}$ of Hogdon 4896 smokeless powder were allowed to equilibrate for one (1) hr. Subsequently, triplicate samples were taken along with a correspondent blank and SPME extraction of the representative odorant was made for five (5) sec at room temperature followed by the desorption of the samples using GC/ECD.

In addition, extraction of signature volatile emanating from TNT explosive material was performed under controlled conditions $\left(20.4{ }^{\circ} \mathrm{C}\right.$ with a relative humidity of $39.0 \%$ ). In order to confirm the presence of 2,4-DNT signature odorant, $25 \mathrm{~g}$ of TNT flakes obtained from the Bureau of Alcohol, Tobacco, Firearms and Explosives (ATF) were placed in each crystal glass container $(8 \mathrm{oz})$ and allowed to reach equilibrium prior to SPME analysis. Solid phase microextraction (SPME) analysis of the sample was 
performed in triplicate and extraction time of one (1) hr was selected in order to preserve volatiles. A blank sample was also analyzed to control for any cross contamination and as a control for proper instrument detection.

*Note: SPME extraction time of TNT was longer (one (1) hr) in order to preserve volatiles since samples were taken in Tennessee.

\section{Nitroglycerin (NG)}

Double based smokeless powder was used to evaluate the target analyte for the nitrate esters class. Thus, nitroglycerin (NG) was determined by mixing $20 \mathrm{mg}$ aliquot of Accurate \#7 double smokeless powder with one (1) ml of acetonitrile for three (3) hours. Eventually, a five (5) $\mu 1$ of this solution was diluted in $995 \mu \mathrm{l}$ of acetonitrile. $200 \mu 1$ of the diluted solution was placed in a two (2) $\mathrm{ml}$ vial and consequently injected for analysis via gas chromatography-electron capture detection $(\mathrm{GC} / \mathrm{ECD})$ for proper identification of NG in the sample (NG is the active ingredient in the double smokeless powder). Proper blank was also injected to control for any cross contamination, and as a control for proper instrument detection.

- Evaluation and optimization of the flow rates (low, medium, and high)

\section{SPME Extraction Time Optimization:}

\section{2-ethyl-1-hexanol (2E1H) and 2, 3-Dimethyl-dinitrobutane (DMNB)}

*Note: Due to limitations of availability of C4 explosive material, SPME extraction time optimization of both representative odorants from C4: 2-ethyl-1-hexanol $(2 \mathrm{E} 1 \mathrm{H})$ and 2, 3-Dimethyl-dinitrobutane (DMNB) was performed in the laboratory by direct analysis of 
the chemicals compounds. In the case of $2 \mathrm{E} 1 \mathrm{H}$, analysis was performed by using controlled odor mimic permeation system (COMPS). Since 2E1H compound shows high volatility (Table 5) and exhibits an extremely strong odor; in order to deliver the known target vapor flux in an instant and reproducible manner COMPS were created. COMPS refer to a new technique in which target odors can be stored inside a permeable package such as low density polyethylene and heat sealed. The use of COMPS will allow the preequilibration of the target odors inside the package prior sampling. Low density two (2) mil polyethylene bags were chosen for the preparation of these COMPS because this simple structure is comprised of a long chain of carbon atoms in which the two hydrogen atoms attached to each carbon atom have been substituted by additional polyethylene chains creating a branching polymer, which permits the easy loss of volatile compounds. In addition, low density polyethylene (LDPE) bags have less density and higher ductility that allows better and faster dissipation rates. Therefore, in this experiment COMPS of 2E1H were made and placed inside the crystal jar for subsequent HSCS collection. On the other hand, for DMNB analysis, pure compound was directly used. In order to determine the optimal SPME extraction time for analysis of $2 \mathrm{E} 1 \mathrm{H}$ and DMNB active odorants from C4 explosive material, a study of different extraction times was performed by analyzing $2 \mathrm{E} 1 \mathrm{H}$ and DMNB chemical compounds individually.

\section{2-ethyl-1-hexanol (2E1H)}

In this procedure, $1 \mathrm{~mL}(0.833 \mathrm{~g})$ of liquid compound $2 \mathrm{E} 1 \mathrm{H}(>99.6 \%$ obtained from Sigma-Aldrich (St Luis, MO)) was spiked onto 2" x 2" sterile gauze pads and heat sealed within two (2) mil LDPE (low density polyethylene) bag. Triplicate samples were 
prepared along with a correspondent blank in which the gauze pad with no compound was also heat sealed within the LPDE bag. Samples were collected at indoor laboratory conditions $\left(22.2^{\circ} \mathrm{C}\right.$ with a relative humidity of $48.3 \%$ and each bag was placed inside crystal jars (8 oz) and allowed to equilibrate for at least one (1) hour. Immediately after equilibrium was reached, HSCS collection of the chemical compound was made onto the sorbent material at medium flow rate for $30 \mathrm{sec}$ which are the default settings of the device following the same protocol explained in section 5.3.2 (see Figure 8). To collect data for this analysis, SPME extraction was made at time intervals of 5, 15, 30, 45, and $60 \mathrm{~min}$ in which triplicate samples and a blank were taken for each of the selected time intervals. Immediately after HSCS collection, samples were placed in a hot plate at about $56^{\circ} \mathrm{C}$ followed by GC/MS analysis to profile the collected odor.
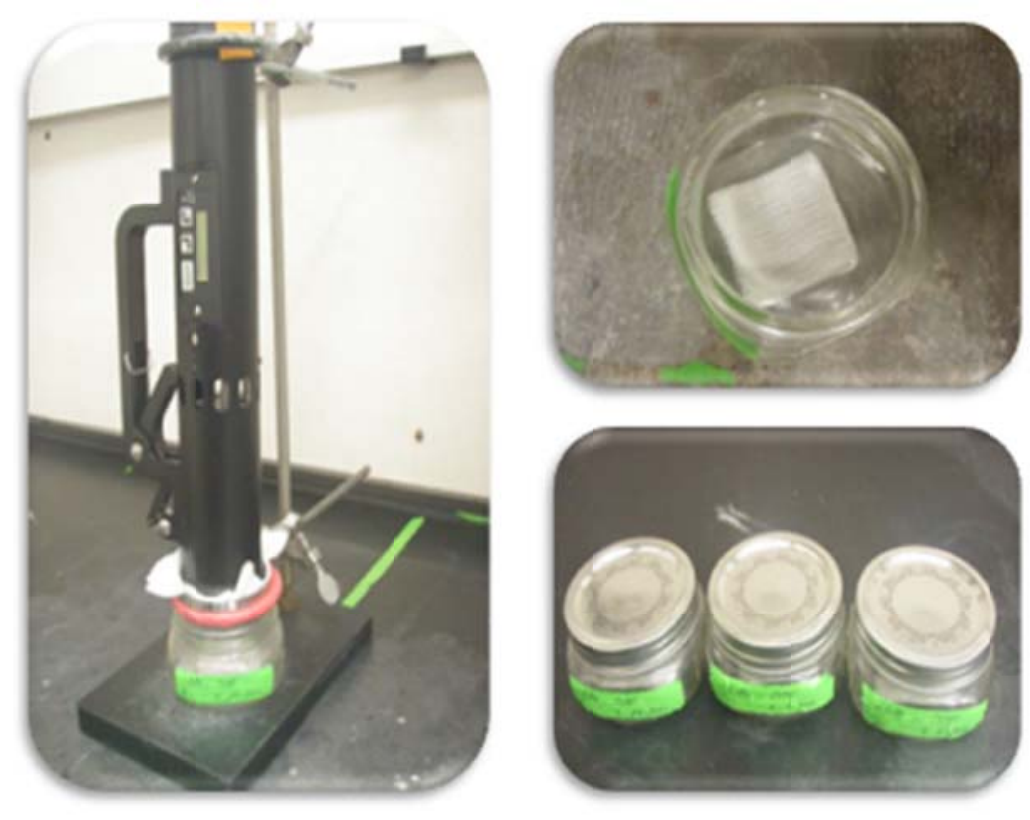

Figure 8: HSCS Collection of 2E1H Chemical Compound 


\section{2, 3-Dimethyl-dinitrobutane (DMNB)}

The study for optimal SPME extraction time for DMNB was performed by analyzing DMNB chemical compound 98\% obtained from Aldrich (St Luis, MO). In this procedure, three samples of the pure solid compound with an average mass of $3 \mathrm{~g}$ were placed each in three different crystal jars $(8 \mathrm{oz})$. Triplicate samples along with a blank (empty jar) were made at indoor laboratory conditions $\left(21.5^{\circ} \mathrm{C}\right.$ with a relative humidity of 49.4\%) and allowed to equilibrate for at least one (1) hr. Immediately after equilibrium was reached, HSCS collection of the DMNB chemical compound was made onto the sorbent material at medium flow rate for $30 \mathrm{sec}$ (Figure 9) following the same protocol for collection of $2 \mathrm{E} 1 \mathrm{H}$ as explained above.
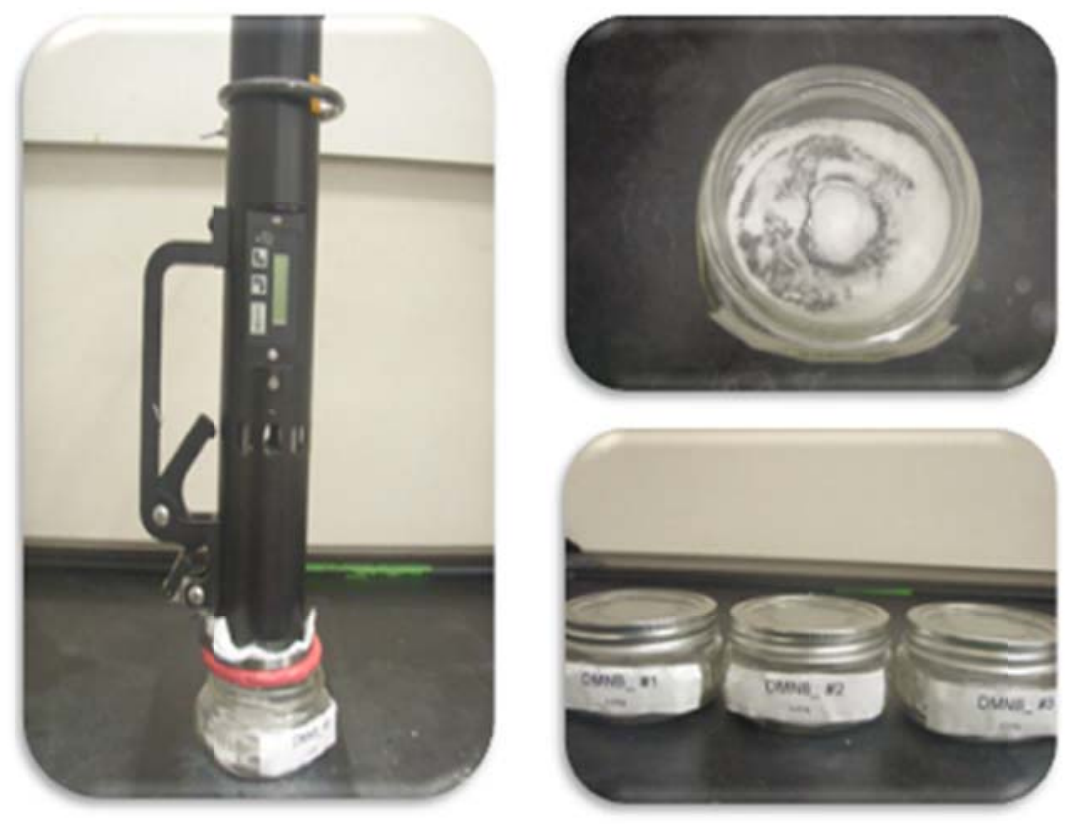

Figure 9: HSCS Collection of DMNB Chemical Compound 


\section{2, 4- Dinitrotoluene (2, 4 -DNT)}

In an effort to determine the optimal SPME extraction time of 2, 4-DNT active odorant, a study of different extraction times was performed. HSCS collection was performed on the same samples utilized for identification of signature odorant at indoor laboratory conditions $\left(21.8{ }^{\circ} \mathrm{C}\right.$ with a relative humidity of $\left.53.6 \%\right)$ at medium flow rate for $30 \mathrm{sec}$ (default settings) following the HSCS protocol for sample collection (section 5.3.2). Immediately after HSCS collection, the samples were stored in silanized $40 \mathrm{ml}$ glass vials and placed in a hot plate at about $56{ }^{\circ} \mathrm{C}$ followed by GC/ECD analysis to profile the collected odor. Analyses were taken at 1, 3, 5, 7, 15, and 21 hours intervals and triplicate samples for each time interval were taken along with a correspondent blank.

\section{Nitroglycerin (NG)}

In order to establish the optimal SPME extraction time of nitroglycerin (NG) active odor from double based smokeless powder, a study of different extraction times was performed. Samples were collected at indoor laboratory conditions $\left(20.1^{\circ} \mathrm{C}\right.$ with a relative humidity of $48.3 \%$ ) by placing approximately $150 \mathrm{~g}$ of double based smokeless powder in each $8 \mathrm{oz}$ crystal jar and allowed to reach equilibrium for $24 \mathrm{hr}$ in order to obtain a homogeneous distribution of the odor. Immediately after equilibrium was reached, HSCS collection of target odorant was made at medium flow rate for $30 \mathrm{sec}$, which are the default settings of the device by following the HSCS protocol for sample collection (section 5.3.2). To collect data for this analysis, a sample along with its correspondent blank was taken for each extraction time. Analyses were made at intervals 
of $1,3,5,7,15$, and $21 \mathrm{hr}$. Immediately after HSCS collection, samples were placed in a hot plate at about $50^{\circ} \mathrm{C}$ followed by $\mathrm{GC} / \mathrm{ECD}$ analysis to profile the collected odor.

\section{Flow Rate optimization}

2-ethyl-1-hexanol (2E1H) and 2, 3-Dimethyl-dinitrobutane (DMNB)

The HSCS flow rate optimization utilized samples obtained from Metro Dade K9 facility (source \#2) and from the ATF (source \#3). The samples from Metro Dade K9 facility were too big to fit the glass containers $(8 \mathrm{oz})$ used for HSCS collection. Therefore, one of the samples was cut in three (3) small pieces of similar amounts with a mass average of $98 \mathrm{~g}$. The samples obtained from ATF had an average mass of $70 \mathrm{~g}$. Previous dynamic collection, the samples were placed in three different crystal jars (8 oz) for at least one (1) hr in order to obtain a homogeneous headspace distribution of the target odor. Triplicates samples were collected at low, medium, and high flow rates for $30 \mathrm{sec}$ by following the protocol for HSCS collection (section 5.3.2). Temperature and relative humidity was recorded. The samples were stored in silanized $40 \mathrm{ml}$ glass vials after collection was completed. Immediately after collection, vials were placed in a hot block at about $56{ }^{\circ} \mathrm{C}$. SPME extraction was then conducted for 30 min followed by GC/MS analysis to obtain the headspace profile of the collected active odor from $\mathrm{C} 4$ explosive material.

The samples obtained from all three sources were eventually utilized for HSCS collection at the optimal flow rate. The samples obtained from City of Miami Police (source \#1) had an average mass of $157 \mathrm{~g}$. 


\section{2, 4- Dinitrotoluene (2, 4 -DNT)}

To establish the optimal flow rate for the HSCS collection of 2,4-DNT active odor, the same samples of Hogdon 4896 smokeless powder ( utilized for SPME optimization as explained above) were used to make a comparison of the three different flow rates low, medium, and high. A total of three (3) trials were conducted with triplicate samples per trial session (total of nine (9) samples) for each flow rate and each trial was conducted on different days with a corresponding blank sample to monitor for any background/contamination issues. The samples were collected following the HSCS protocol (section 5.3.2) on different days indoors in which temperature and relative humidity was recorded. The samples were stored in silanized $40 \mathrm{~mL}$ glass vials after collection. Immediately, vials were placed in a hot block at a temperature of $56{ }^{\circ} \mathrm{C}$. SPME extraction was then conducted for $21 \mathrm{hr}$ followed by GC/ECD analysis to obtain the headspace profile of the collected active odor.

In addition, TNT explosive material (obtained from the Bureau of Alcohol, Tobacco, Firearms, and Explosives (ATF) in Tennessee) only a set of triplicate samples was taken for this study. Triplicate samples were made to be analyzed at all flow rates at 30 sec. HSCS and SPME procedures are the same as in the evaluation of signature volatiles from explosive families section explained above

\section{Nitroglycerin (NG)}

For the HSCS collection of nitroglycerin in double based smokeless powder, the same samples utilized for optimization of SPME extraction time (150 g of double based smokeless powder in 8oz crystal jars ) were used for HSCS collection at low, medium, 
and high flow rates for $30 \mathrm{sec}$ following the HSCS protocol explained in 5.3.2. A total of three (3) trials were conducted with triplicate samples per trial session (total of nine (9) samples) for each flow rate and each trial was conducted on different days with a corresponding blank sample to monitor for any background/contamination issues. The samples were stored in silanized $40 \mathrm{~mL}$ glass vials after collection was completed and placed in a hot block at about $50{ }^{\circ} \mathrm{C}$. SPME extraction was then conducted for $21 \mathrm{hr}$ followed by GC/ECD analysis to obtain the headspace profile of the collected active odor.

- Evaluation and optimization of sampling time ( $30 \mathrm{sec}$ vs. $60 \mathrm{sec}$ )

\section{2-ethyl-1-hexanol (2E1H) and 2, 3-Dimethyl-dinitrobutane (DMNB)}

Samples form City of Miami Police and Metro Dade K9 facility (source \# 1 and \#2) were utilized for HSCS collection at optimal established parameters in which a total of three (3) trials were conducted with triplicate samples per trial session (total of nine (9) samples) and each trial was conducted on different days with a corresponding blank by following the same SPME procedure for flow rate optimization as explained above.

Samples from ATF (source \#3) were included but only a set of triplicates was taken. Thus, only triplicates were utilized for comparison purposes with samples obtained from the other two sources (City of Miami Police and Metro Dade K9 facility).

\section{2, 4- Dinitrotoluene (2, 4 DNT)}

For HSCS sampling time optimization of single based smokeless powder, the same samples and same SPME procedure utilized for flow rate optimization was followed. 
Triplicate samples of TNT explosive material (obtained from ATF) were taken to be analyzed at all flow rates at 60 seconds. Since TNT flakes present a different matrix than smokeless powder and instrumental analysis could not be made after collection of samples, optimization of 2, 4 DNT odorant from TNT at all flow rates and times was necessary. HSCS and SPME procedures are the same as in the evaluation of signature volatiles from explosive families section explained before.

\section{Nitroglycerin (NG)}

For HSCS sampling time optimization of double based smokeless powder, the same samples and the same SPME procedure utilized for flow rate optimization was followed (section 5.6).

- Comparison of Collection Modes: Static Vs. Dynamic

\section{2-ethyl-1-hexanol (2E1H) and 2, 3-Dimethyl-dinitrobutane (DMNB)}

Static analysis of $\mathrm{C} 4$ explosive material was performed in time intervals of $0.5,1$, $5,15,30,45,60,90$, and 120 min. Samples were taken in triplicate for each of the selected time intervals and each trial was conducted on different days with a corresponding control sample to monitor for any possible background/contamination.

The samples were collected indoors $\left(23.8^{\circ} \mathrm{C}\right.$ with a relative humidity of $\left.77.2 \%\right)$ at Metro Dade K9 facility (source \#2). The collection material was a 4" x 4" cotton gauze pad and all samples were stored in silanized $40 \mathrm{~mL}$ glass vials. After samples arrived to the lab, vials were injected via SPME for $30 \mathrm{~min}$ at about $56^{\circ} \mathrm{C}$ and subsequently analyzed by GC/MS in order to characterize the accumulated volatile organic compounds. 


\section{2, 4- Dinitrotoluene (2,4 DNT)}

Solid phase microextraction (SPME) of 2,4 DNT representative volatile from single based smokeless powder was conducted for $21 \mathrm{hr}$ in a hot plate at about $56{ }^{\circ} \mathrm{C}$ followed by $\mathrm{GC} / \mathrm{ECD}$ analysis to obtain the headspace profile of the collected odorant. Static analysis was performed on the same samples utilized in previous task in time intervals of $0.5,1,5,15,30,45,60,120$, and $240 \mathrm{~min}$. The samples were taken in triplicate for each of the selected time intervals and each trial was conducted on different days with a corresponding control sample to monitor for any background/contamination issues. The samples were collected at indoor laboratory conditions $\left(22.4{ }^{\circ} \mathrm{C}\right.$ with a relative humidity of $46.5 \%)$.

\section{Nitroglycerin (NG)}

Solid phase microextraction (SPME) of NG representative odorant from double based smokeless powder was conducted for 21 hours in a hot plate at about $50{ }^{\circ} \mathrm{C}$ followed by GC/ECD analysis to obtain the headspace profile of the collected odorant Static mode collection was performed on the same samples utilized for flow rate and time sampling optimization in time intervals of $0.5,1,5,15,30,45(\mathrm{~min}), 60(1 \mathrm{hr}), 120(2 \mathrm{hr}), 240(4$ hr), $420(7 \mathrm{hr}), 900(15 \mathrm{hr})$, and $1260 \mathrm{~min}(21 \mathrm{hr})$. The samples were taken in triplicate for each of the selected time intervals and each trial was conducted on different days with a corresponding control sample to monitor for any background/contamination issues. The samples were collected at indoor laboratory conditions $\left(24.4{ }^{\circ} \mathrm{C}\right.$ with a relative humidity of $50.6 \%)$. 
- Evaluation of longevity of sorbent materials

\section{Experimental Procedure for Persistence of C-4 Explosive Representative Volatiles}

In the present study, explosive material was obtained from City of Miami Police (Source \# 1). The storage containment study was done over distinctive time periods, namely after one (1), four (4), 24, 168 (one week), 336 (two weeks), and 672 hours (three weeks) followed by SPME extraction to evaluate instrumental response. The optimal HSCS air flow rate and time settings obtained from Subtasks 2 and 3 (high flow rate and $60 \mathrm{sec})$ were used for the sample collection. The samples were collected from C4; the odor source for the HSCS dynamic airflow collection of two representative volatiles: 2E1H and DMNB. In order to proceed with HSCS sampling collection, three (3) samples of $\mathrm{C} 4$ material with a total average mass of $157 \mathrm{~g}$ were separately placed in a crystal container ( $8 \mathrm{oz})$ and were allowed one (1) $\mathrm{hr}$ to equilibrate, the process allows a homogeneous distribution of the target odorant inside the crystal jar. The same HSCS protocol for the dynamic collection of $2 \mathrm{E} 1 \mathrm{H}$ and $\mathrm{DMNB}$ odorants was followed as explained in section 5.3.2.

In order to determine the optimal storage container, a comparison of three (3) different storage systems: glass vials, aluminum bags (heat sealed), and ziploc (double zipper) bags was performed (see Figure 10). Subsequently, for each of the three (3) different storage container types, a set of triplicate samples from $\mathrm{C} 4$ explosive material (containing both representative odorants) was taken to be analyzed over a period of a monthe along with a blank sample to control for any cross contamination and as a control for proper instrument detection. 


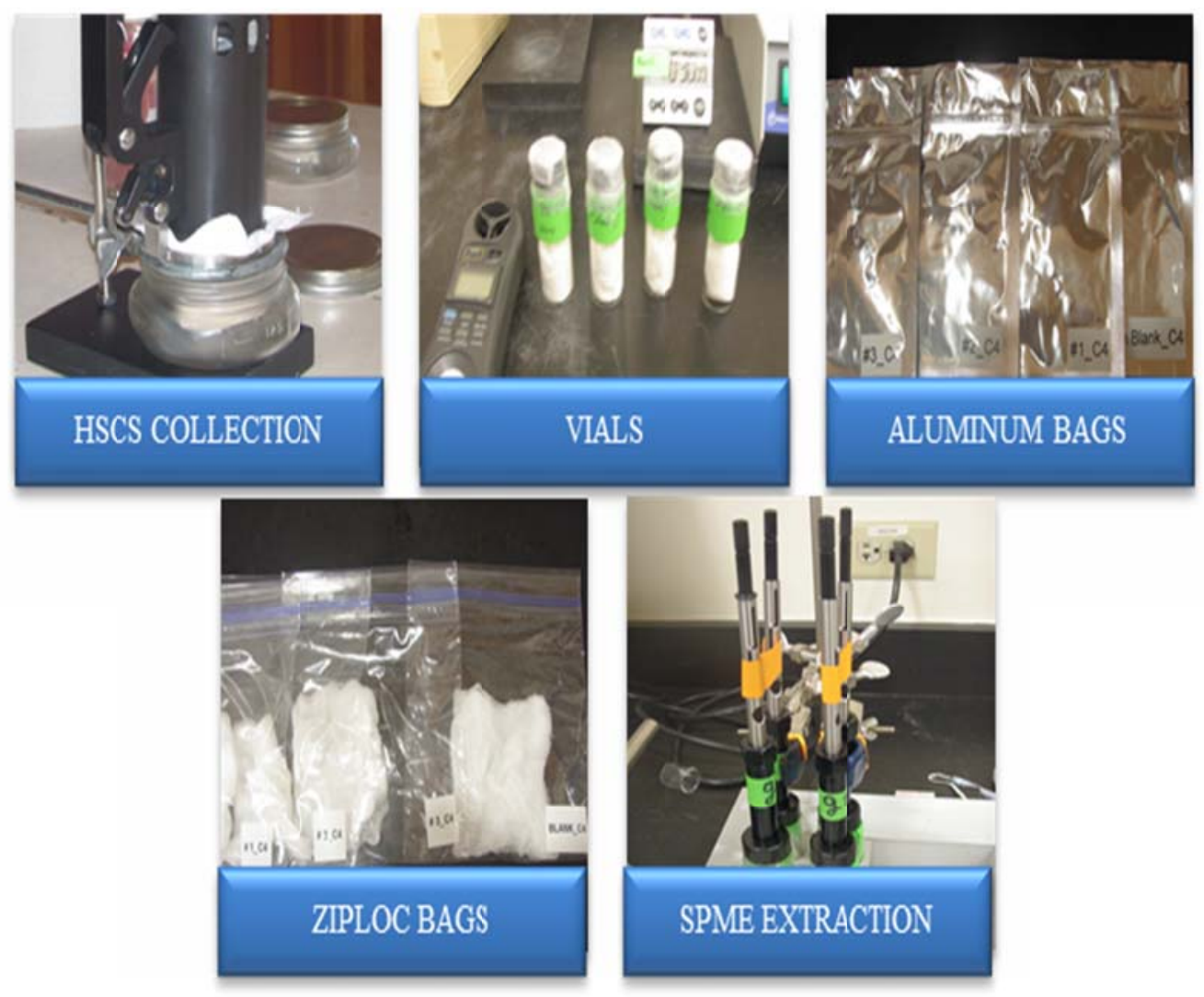

Figure 10: Storage Containment Systems for Collected HSCS Samples

The samples of $\mathrm{C} 4$ explosive material were collected in triplicate along with a blank at atmospheric conditions $\left(22.2{ }^{\circ} \mathrm{C}\right.$ with a relative humidity of $\left.66.1 \%\right)$. Eventually, the gauze pads were stored in $40 \mathrm{ml}$ airtight glass vials. After one (1) hr, SPME extraction at $56{ }^{\circ} \mathrm{C}$ was conducted for $30 \mathrm{~min}$ followed by GC/MS analysis to profile the collected volatiles. After SPME/GC-MS analysis was performed, glass vial samples were sealed with paraffin and stored in the dark at room temperature for future analysis. Subsequently, the same SPME/GC-MS procedure was repeated for the remaining time intervals of the study. 
The samples of $\mathrm{C} 4$ explosive material utilized for the analysis of aluminum and ziploc bags were collected on different days at atmospheric conditions $\left(28{ }^{\circ} \mathrm{C}\right.$ with a relative humidity of $73.0 \%$ ). A set of triplicates and a blank were taken, and upon completion, the gauze pads were stored inside the bags (aluminum bags were heat sealed and ziploc bags double zipper). After one (1) hour, gauze pads were transferred from the bags to $40 \mathrm{ml}$ glass vials and extracted for $30 \mathrm{~min}$ at $56{ }^{\circ} \mathrm{C}$ using SPME. Immediately after extraction, the headspace profile of the $2 \mathrm{E} 1 \mathrm{H}$ and DMNB VOCs was obtained by GC/MS. After SPME analysis was performed, gauze pads were transferred back to the bags (those transferred to the aluminum bags were heat sealed) and stored in the dark at room temperature to eliminate access of light that could degrade the VOCs. This procedure was repeated for each of the storage time periods being evaluated for this research task.

In order to simulate the exposure of the gauze pads to any environmental changes that would occur during actual field use and its effects during transferring from aluminum and ziploc bags to vials (for SPME extraction); all storage containers with the gauze pad were opened once a week for five (5) min to simulate the opening and closing of these pads while being used in real canine training field practices.

\section{Experimental Procedure for Persistence of Smokeless Powder Signature Volatiles}

The storage containment study was done in time intervals of one (1) hr, four (4) hr, $24 \mathrm{hr}$, one week (7 days), two weeks (14 days), and four weeks (28 days). In this part of the study, same samples of single and double based smokeless powders were used as the odor source and dynamic airflow collection was completed by using the optimal air flow 
rate and time settings obtained from tasks 2 and 3. The results obtained in these tasks determined that high flow rate and $30 \mathrm{sec}$ were the optimal parameters for HSCS collection of target volatiles from smokeless powder.

In order to determine the optimal storage container, a comparison of three (3) different storage systems: glass vials, aluminum bags (heat sealed), and ziploc (double zipper) bags was performed. For each of the three (3) different storage containers, only one (1) set of triplicate samples from each of the representative odorants from single based and double based smokeless powders was taken to be analyzed at all-time intervals along with a blank sample to control for any cross contamination and as a control for proper instrument detection.

The samples from double based smokeless powder were collected in triplicate along with a blank at atmospheric conditions $\left(26.4{ }^{\circ} \mathrm{C}\right.$ with a relative humidity of $\left.51.8 \%\right)$. After collection, the gauze pads were stored in $40 \mathrm{~mL}$ airtight glass vials. After one (1) hr, vials were placed on a hot block at $50{ }^{\circ} \mathrm{C}$ and SPME extraction was then conducted for $21 \mathrm{hr}$ followed by GC/ECD analysis with five (5) min desorption time to obtain the headspace profile of the NG active odorant. After SPME analysis, glass vial samples were sealed with paraffin and stored in the dark at room temperature for future analysis. Subsequently, the same SPME/GC-ECD procedure was repeated for the remaining time intervals of the study.

The samples from double based smokeless powder in aluminum and ziploc bags were collected at atmospheric conditions $\left(26.3{ }^{\circ} \mathrm{C}\right.$ with a relative humidity of $\left.53.5 \%\right)$. After collection, the gauze pads were stored inside the bags (aluminum bags were heat sealed). After one (1) hr, gauze pads were transferred from the bags to $40 \mathrm{~mL}$ glass vials 
and immediately placed on a hot block at $50{ }^{\circ} \mathrm{C}$ and SPME extraction was then conducted for $21 \mathrm{hr}$ followed by GC/ECD analysis with five (5) min desorption time to obtain the headspace profile of the NG active odorant. After SPME analysis, gauze pads were transferred back to the bags (those transferred to the aluminum bags were heat sealed) and stored in the dark at room temperature to eliminate access of light that could degrade the VOC's. Eventually, the same transferring of gauze pads from aluminum and ziploc bags to glass vials as well as SPME/GC-ECD procedure was repeated for each of the storage time periods evaluated for this task.

The samples from single based smokeless powder were collected at atmospheric conditions $\left(25.5^{\circ} \mathrm{C}\right.$ with a relative humidity of $\left.55.2 \%\right)$ and the same protocol for storage and gauze pad transferring to glass vials, aluminum bags, and ziploc bags used for double based smokeless powder was followed for the remaining time intervals. The samples were placed on a hot block at $56{ }^{\circ} \mathrm{C}$ and SPME extraction was conducted for $21 \mathrm{hr}$ followed by GC/ECD analysis with five (5) min desorption time to obtain the headspace profile of the 2,4-DNT active odorant.

In addition, all storage containments with the gauze pad were opened once a week for five (5) min as it was performed for analysis of samples from C4 explosive material.

- Field evaluation of laboratory HSCS training aids

\section{Field trials}

Field evaluations were performed on site at Camp Pendleton, California in collaboration with certified canine teams of the United States Marine Corps. The test was conducted in blind manner and the trials were performed in condemned barrack units (no 
longer in use) in which four (4) explosive families were evaluated from three (3) different explosives: C4 (nitro-alkanes and nitro-amine), detonation cord (nitrate ester), TNT (nitro-aromatic). Canine teams were allowed to complete the search of rooms in two passes. The first pass being conducted by the canine on his own (off-leash) and if handler considered it necessary, the team would detail the room on the second pass with the canine on-leash.

As depicted in Figure 11, each room had six (6) possible locations and each location had a possible number of hides (showed in parenthesis). Each room contained only one hide and the selection of hides was performed by using dice rolls to determine which explosive was to be used, to select the piece of furniture to place the hide in, and to choose where in the furniture the hide would be placed. As soon as all hides were in place, explosives and/or HSCS samples were allowed to sit for at least 30 minutes before the beginning of the test.

\section{HSCS Sample Collection}

Samples were collected indoors at atmospheric conditions of $28.5^{\circ} \mathrm{C}$ and a relative humidity of $53.0 \%$. Each type of explosive material was placed in different crystal jars and allowed to equilibrate prior HSCS dynamic collection as seen in Table 4. 


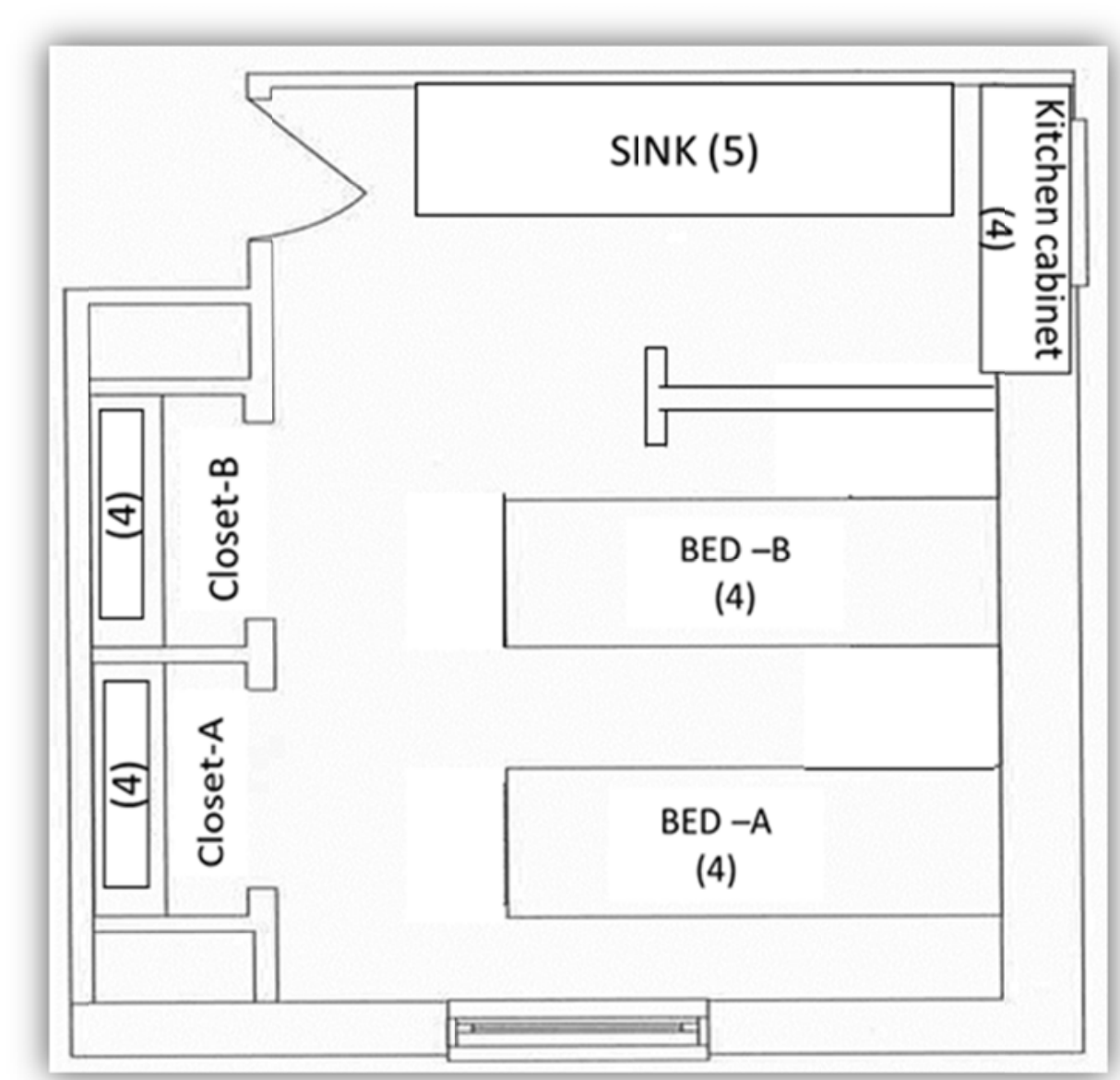

Figure 11: Test Room Diagram

Table 4: Evaluated Parameters for Selected Explosive Material

\begin{tabular}{ccccc}
\hline Explosive & Weight & $\begin{array}{c}\text { Equilibrium } \\
\text { Time }\end{array}$ & Flow Rate & $\begin{array}{c}\text { Collection } \\
\text { Time } \\
\text { (sec) }\end{array}$ \\
\hline $\mathrm{C} 4$ & $1 / 4 \mathrm{lb}(1)$ & $2 \mathrm{hrs}$ & $\mathrm{HIGH}$ & 60 \\
\hline Det Cord & $1 \frac{1}{2} \mathrm{ft}(2)$ & $3 \mathrm{hrs}$ & $\mathrm{HIGH}$ & 30 \\
TNT & $1 / 4 \mathrm{lb}(1)$ & $3 \mathrm{hrs}$ & LOW & 60 \\
\hline
\end{tabular}




\section{Aged Samples}

Aged samples were collected three (3) weeks before canine testing (according to the time in which samples were no longer instrumentally detected established in the previous task). After samples were dynamically collected at ambient conditions at the optimal HSCS parameters, they were stored in $40 \mathrm{ml}$ glass vials and sealed with parafilm in order to preserve the integrity of the volatiles. The purpose of this task was to evaluate the optimal storage time period of HSCS training aids and canines' ability to recognize the odor even though VOCs were no longer detected by analytical instruments. For this stage, search was conducted in five (5) rooms including a positive control (1 lb. single based smokeless powder) and a blank room. To guarantee that rooms were clear of any unwanted interfering odorants, a proofing dog was used to search all selected locations before the trial. After using the dice rolls, hides were placed in their designated areas and left for at least $30 \mathrm{~min}$ prior to the beginning of the trial to ensure proper release of volatiles.

\section{Fresh Samples}

Fresh samples dynamically collected at optimal parameters (previously established from the selected explosive material) were stored in $40 \mathrm{ml}$ glass, sealed with parafilm, and kept in a box at ambient conditions until the following day. Only eleven (11) canines were used in this trial since one canine was disabled (due to heat exhaustion). In this stage, five (5) rooms including a positive control (1 lb. single based smokeless powder) and a blank room were used for search purposes. In addition, to guarantee that rooms were clear of any unwanted interfering odorants, a proofing dog was used to search all 
selected locations before the trial. After using the dice rolls, hides were placed in their designated areas and left for at least 30 min prior to the beginning of the trial to ensure proper release of volatiles.

\section{RESULTS}

\subsection{TASK 1: EVALUATION OF EXPLOSIVE FAMILIES}

The aim of this task is to evaluate the representative odor signatures of the four main explosive families (see Table 5). Of the four families evaluated, suitable odors have been determined to be 2,3-dimethyl-dinitrobutane (DMNB) a tagging agent representing the nitro alkanes and 2-ethyl-1-hexanol $(2 \mathrm{E} 1 \mathrm{H})$, a fatty alcohol used as an additive commonly found in conjunction with trinitro-triazacyclohexane (RDX) and tetranitrotetrazacyclooctane (HMX) representing the nitro amines. DMNB and 2E1H, both present in plastic bonded explosives (PBX) such as composition 4 (C4). 2, 4- dinitrotoluene (2,4DNT), a plasticizer representing the nitro aromatics present in trinitrotoluene (TNT) and single based smokeless powders. Nitroglycerin (NG), an active explosive ingredient representing the nitrate esters present in double based smokeless powders.

In the present study, real explosive material was analyzed by GC/MS or GC/ECD in order to confirm the presence of each of the representative odorants prior to method optimization and HSCS sampling. Liquid injection was performed for analysis of double based smokeless powder and SPME extraction for the analysis of VOCs extracted from C4 explosive material, single based smokeless powder, and TNT as explained below. 
Table 5: Properties of Different Explosive Families (2)

\begin{tabular}{|c|c|c|c|c|}
\hline $\begin{array}{c}\text { COMPOUNDS \& } \\
\text { EXPLOSIVE } \\
\text { FAMILIES }\end{array}$ & $\begin{array}{c}\text { TYPE OF } \\
\text { MATERIAL }\end{array}$ & $\begin{array}{c}\text { MOLECULAR } \\
\text { WEIGHT } \\
(\mathrm{amu})\end{array}$ & $\begin{array}{c}\text { VAPOR } \\
\text { PRESSURE at } \\
\left(25^{\circ} \mathrm{C}\right) \text { Torr }\end{array}$ & $\begin{array}{c}\text { CHEMICAL } \\
\text { STRUCTURE \& } \\
\text { FORMULA }\end{array}$ \\
\hline $\begin{array}{c}\text { 2E1H } \\
\text { 2-ethyl-1-hexanol } \\
\text { Nitro-amines }\end{array}$ & $\begin{array}{l}\text { Additive in } \\
\text { plastic } \\
\text { explosives: } \\
\text { C4 }\end{array}$ & 130.22 & $1.3 \times 10^{-1}$ & $\mathrm{C}_{8} \mathrm{H}_{18} \mathrm{O}$ \\
\hline $\begin{array}{c}\text { DMNB } \\
\text { 2,3-dimethyl- } \\
\text { dinitrobutane } \\
\text { Nitro-alkanes }\end{array}$ & $\begin{array}{l}\text { Tagging agent/ } \\
\qquad(\mathrm{PBX}) \\
\text { plastic bonded } \\
\text { explosives: } \\
\text { C4 }\end{array}$ & 176.17 & $2.1 \times 10^{-3}$ & $\begin{array}{c}\bigwedge_{0}^{\|} \\
\mathrm{C}_{6} \mathrm{H}_{12} \mathrm{~N}_{2} \mathrm{O}_{4}\end{array}$ \\
\hline $\begin{array}{c}\text { 2,4-DNT } \\
\text { 2-4-dinitrotoluene } \\
\text { Nitro-aromatics }\end{array}$ & $\begin{array}{l}\text { Plasticizer: } \\
\text { TNT and } \\
\text { Single Based } \\
\text { Smokeless } \\
\text { Powder }\end{array}$ & 182.14 & $2.1 \times 10^{-4}$ & $\mathrm{C}_{7} \mathrm{H}_{6} \mathrm{~N}_{2} \mathrm{O}_{4}$ \\
\hline $\begin{array}{c}\text { NG } \\
\text { Trinitroglycerin } \\
\text { Nitrate- esters }\end{array}$ & $\begin{array}{c}\text { Active } \\
\text { ingredient: } \\
\text { Double Based } \\
\text { Smokeless } \\
\text { Powder }\end{array}$ & 227.09 & $2.4 \times 10^{-5}$ & $\mathrm{C}_{3} \mathrm{H}_{5} \mathrm{~N}_{3} \mathrm{O}_{9} \mathrm{O}^{1} \mathrm{~N}_{-}^{+}$ \\
\hline
\end{tabular}




\subsubsection{SPME Extraction and Chromatographic Analysis of Extracted VOCs}

\subsubsection{2-ethyl-1-hexanol (2E1H) and 2, 3-Dimethyl-dinitrobutane (DMNB) Representative Volatiles from $\mathrm{C} 4$}

For the analysis of $\mathrm{C} 4$ explosive material, the presence of both target odorants: 2E1H and DMNB was confirmed in all three samples that were taken from the three different sources. Figure 12, Figure 13, and Figure 14 show the chromatograms of one sample of $\mathrm{C} 4$ explosive material taken at each source. Figure 15 shows the extracted ion chromatogram from one of the samples of $\mathrm{C} 4$ obtained from the City of Miami Police along with a standard. The extracted ions 57 for $2 \mathrm{E} 1 \mathrm{H}$ and 57 and 69 for DMNB were identified by NIST library.

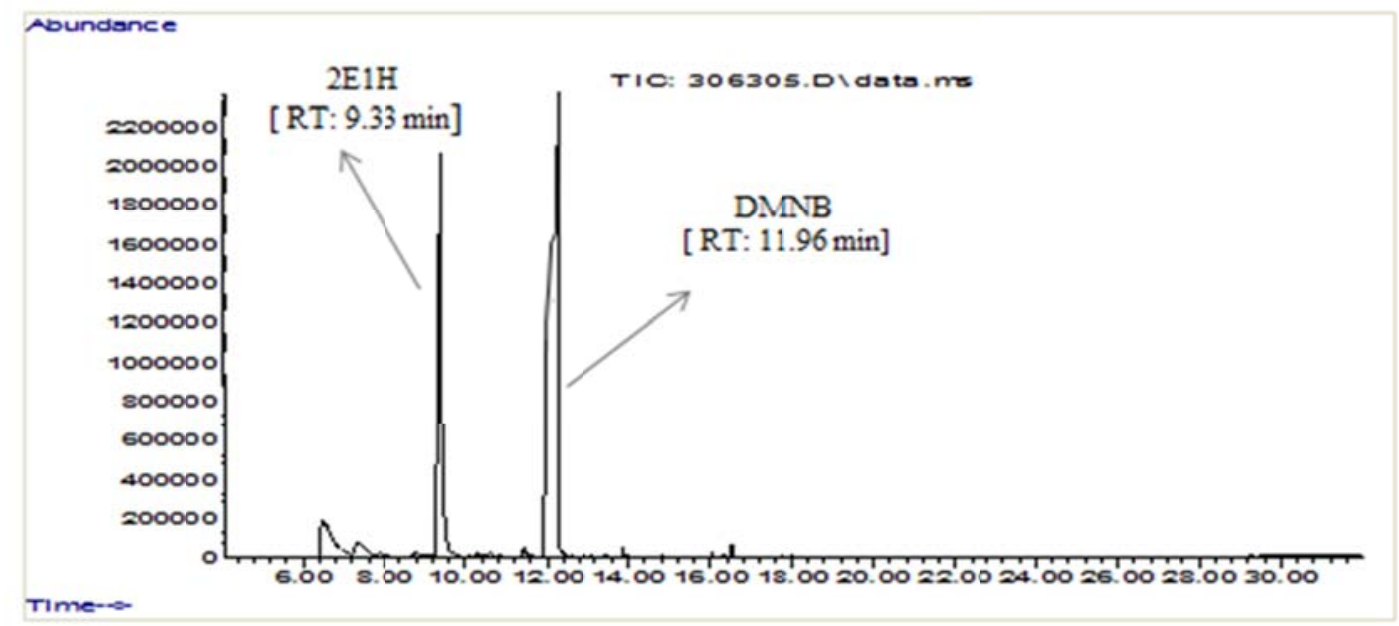

\section{Figure 12: Headspace SPME-GC/MS of Tagged C4 Explosive from City of Miami Police}

According to the results, $2 \mathrm{E} 1 \mathrm{H}$ and DMNB volatiles collected a scent mass average of $163 \pm 5 \mathrm{ng}$ and $350 \pm 7 \mathrm{ng}$ respectively for samples obtained from City of Miami 
Police (Source \#1). The samples from Miami Dade k9 Facility (source \# 2) collected a scent mass average of $34 \pm 5 \mathrm{ng}$ of $2 \mathrm{E} 1 \mathrm{H}$ and $277 \pm 12 \mathrm{ng}$ of DMNB. The triplicate samples form the ATF (source \# 3) collected an average mass of $163 \pm 3 \mathrm{ng}$ of $2 \mathrm{E} 1 \mathrm{H}$ and $203 \pm 8 \mathrm{ng}$ of DMNB with retention time of $10.5 \mathrm{~min}$ and $13.3 \mathrm{~min}$ for $2 \mathrm{E} 1 \mathrm{H}$ and DMNB respectively.

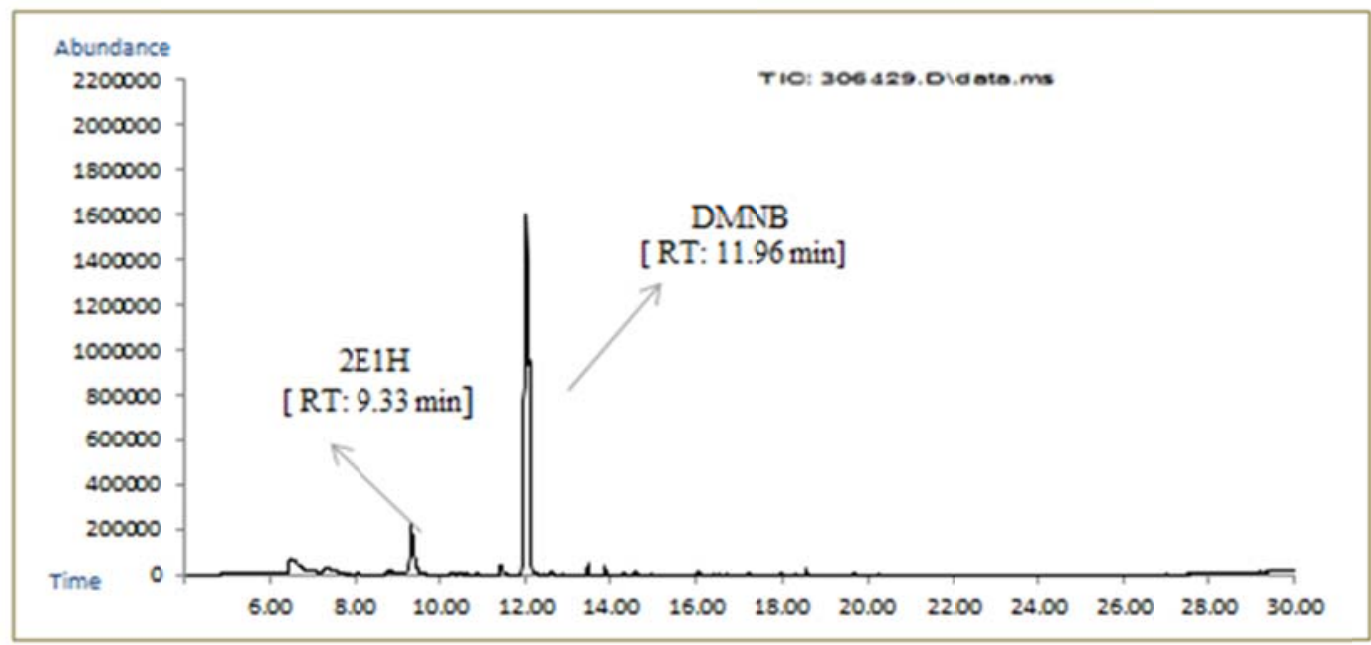

Figure 13: Headspace SPME-GC/MS of Tagged C4 Explosive from Miami Dade k9 Facility

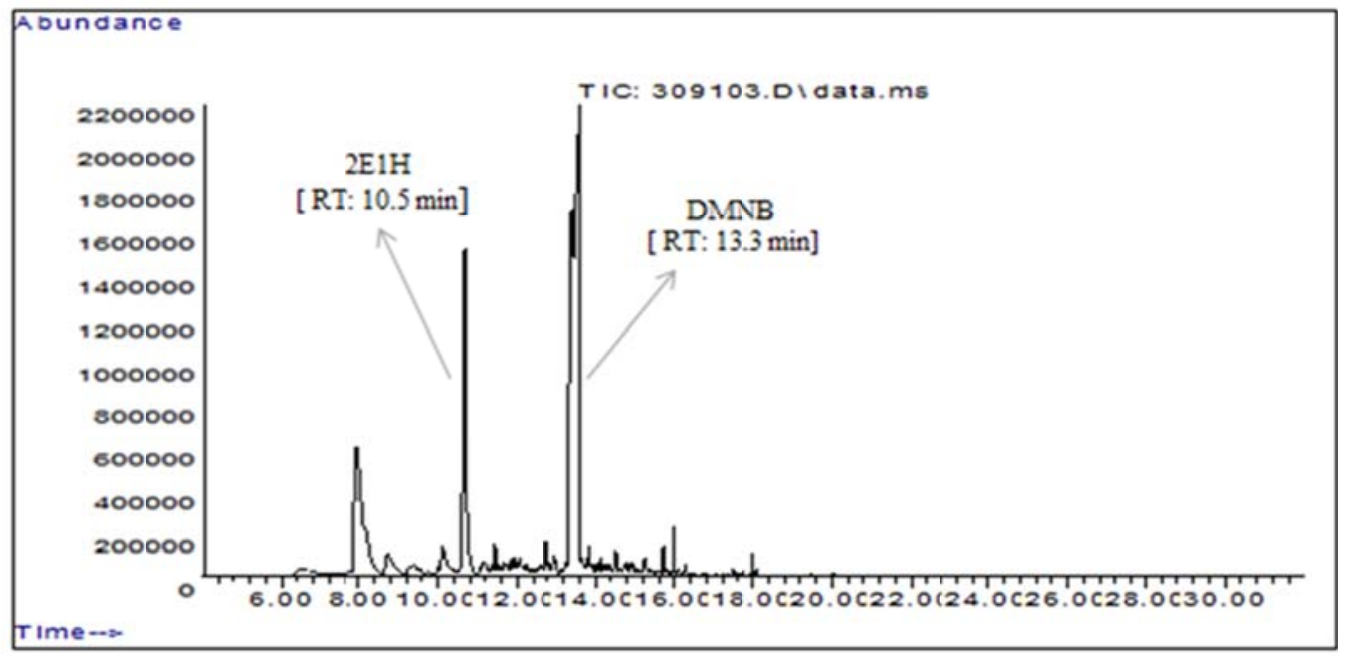

Figure 14: Headspace SPME-GC/MS of Tagged C4 Explosive from the ATF 
*Note: Samples obtained from the ATF (source \# 3) were analyzed at a later time. For this reason, changes in retention time are seen due to method optimization.

As can be observed, target volatiles $2 \mathrm{E} 1 \mathrm{H}$ and DMNB were detected within the headspace of all explosive samples, and therefore meet the quality control for subsequent study with the actual HSCS device.

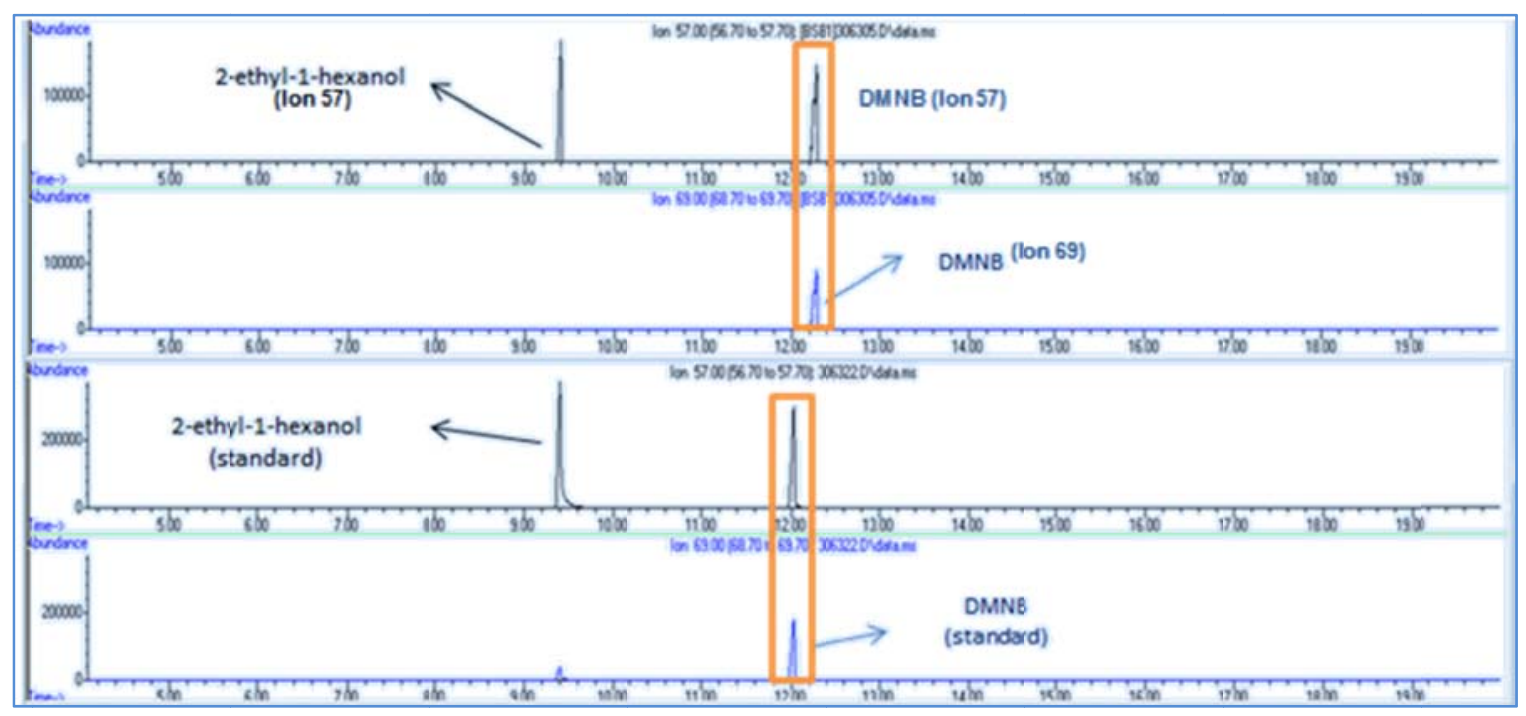

Figure 15: Comparison of Extracted Ion Chromatograms from C4 Signature Odors and Standards Identified by NIST library

\subsubsection{2,4- Dinitrotoluene (2,4 -DNT) Representative Volatile from Single Based Smokeless Powder and TNT}

For the analysis of single based smokeless powder, a scent mass average of $1.30 \pm$ $0.2 \mathrm{ng}$ of 2, 4-DNT volatile was collected from all three (3) samples. The response obtained from one of the single based smokeless powder sample was directly compared with a chemical standard (2ppm) for confirmatory purposes as depicted in Figure 16 (Retention time: $5.30 \mathrm{~min}$ ). 


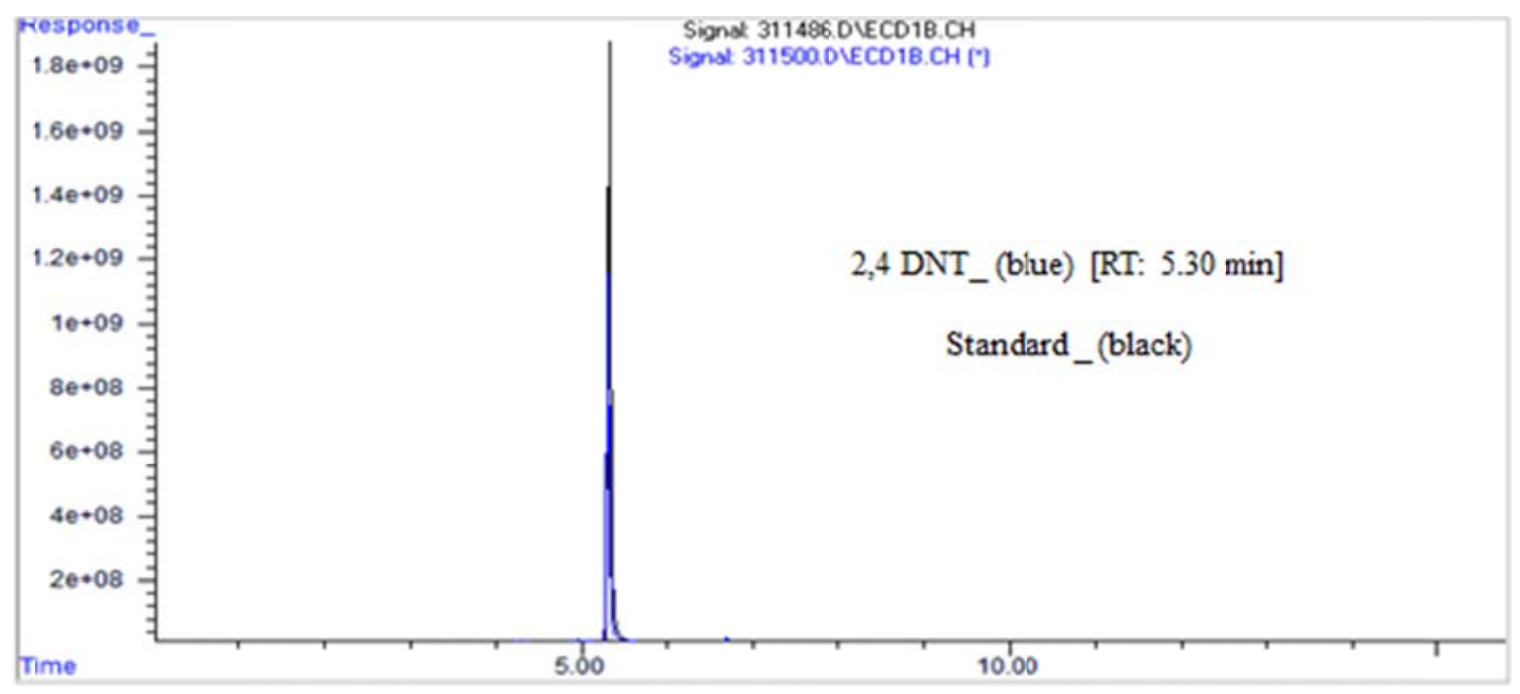

Figure 16: Comparison of 2,4-DNT GC/ECD Detection in Single Based Smokeless Powder and Chemical Standard

The results obtained for the analysis of TNT showed that a scent mass average of $11.84 \pm 0.3 \mathrm{ng}$ of 2,4 -DNT volatile was collected from all three samples. The response obtained from one of the TNT samples was directly compared with a chemical standard (2ppm) for confirmatory purposes as depicted in Figure 17 (Retention time: 5.30 min).

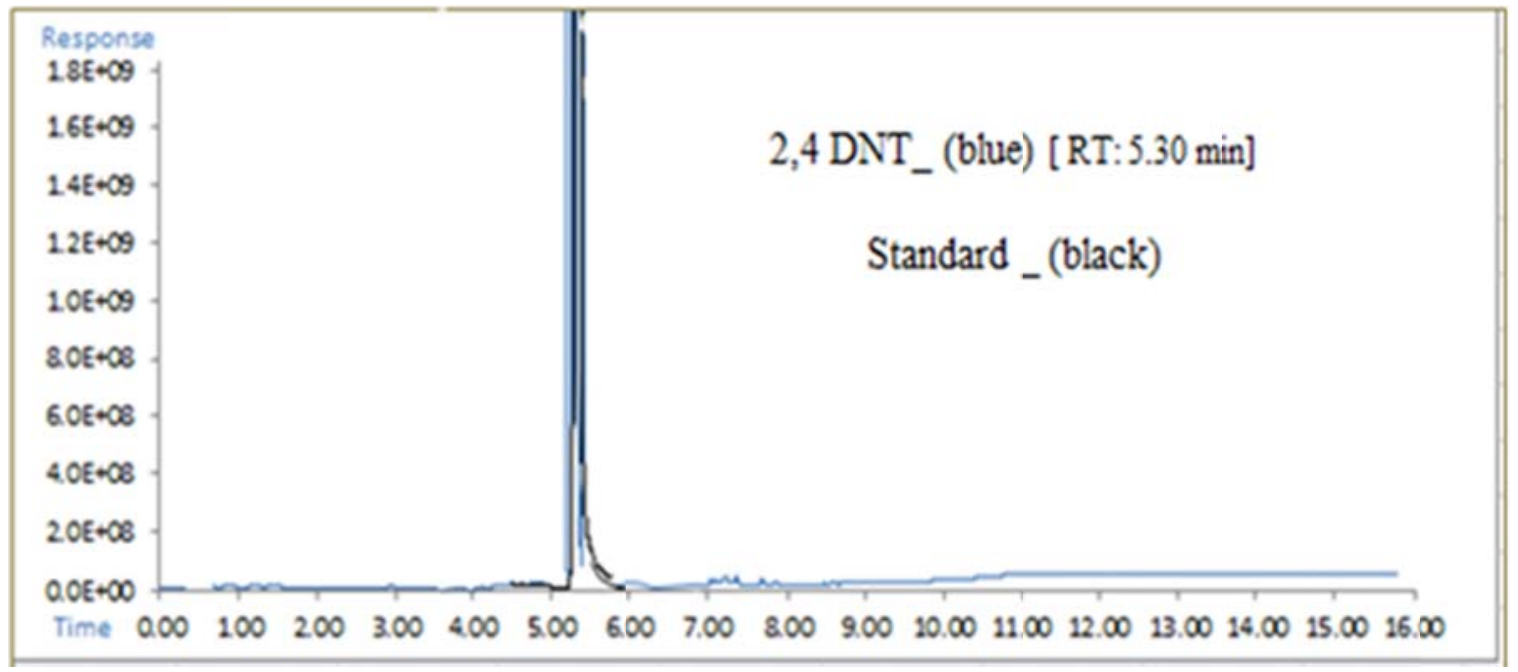

Figure 17: Comparison of 2, 4-DNT GC/ECD Detection in TNT and Chemical Standard 


\subsubsection{Nitroglycerin (NG) Representative Volatile from Double Based Smokeless Powder}

The analysis of double based smokeless powder a scent mass average of $0.72 \pm 0.1 \mathrm{ng}$ of NG volatile was collected from all (3) three samples. The presence of NG in one of the samples was confirmed and the response obtained from the double based smokeless powder sample was directly compared with a chemical standard (1ppm) for confirmatory purposes as shown in Figure 18 (Retention time: $5.90 \mathrm{~min}$ ).

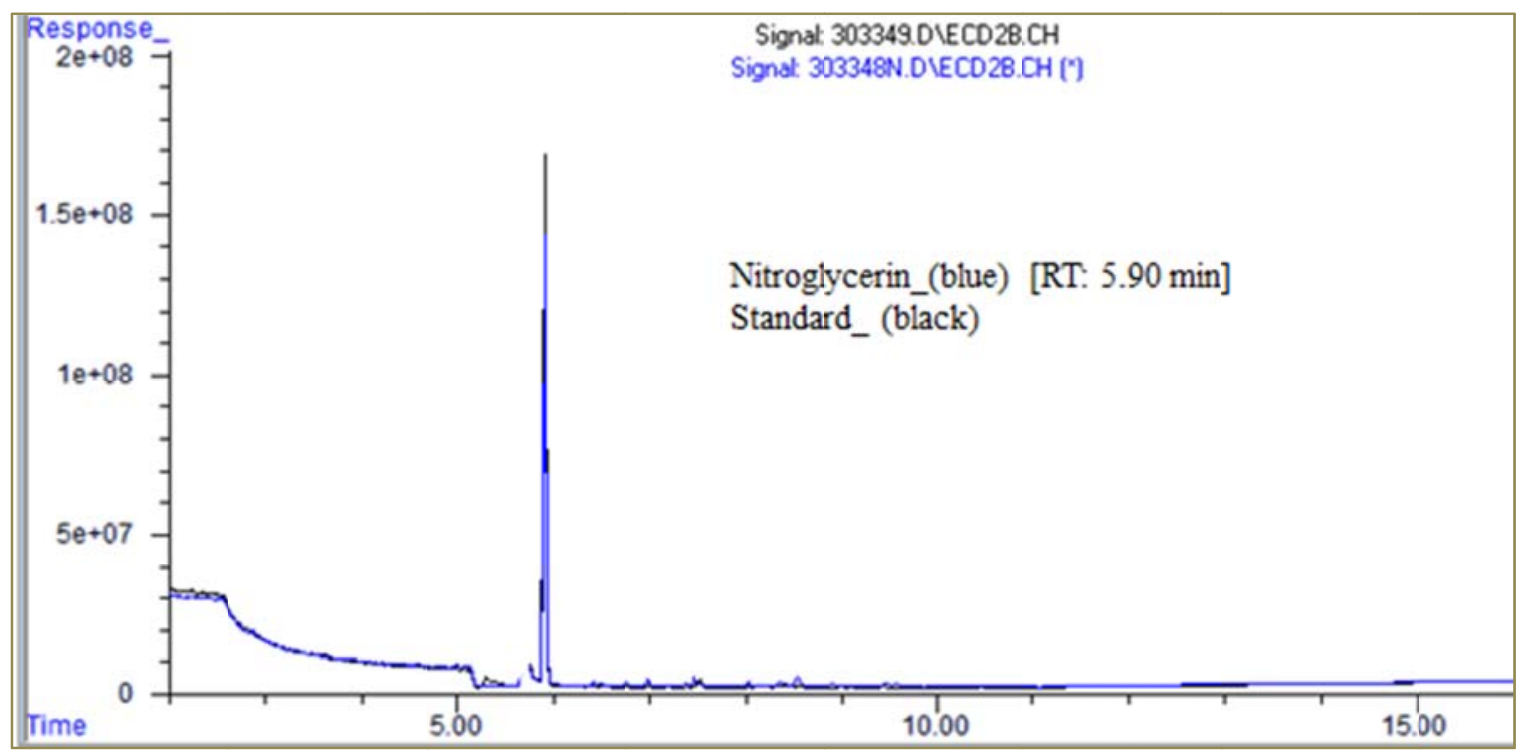

Figure 18: Comparison of NG GC/ECD Detection in Double Based Smokeless Powder and Chemical Standard

\subsubsection{Conclusions}

In order to ensure the quality of research for each task of this study, identification of each target volatile from real explosive materials was absolutely necessary previous dynamic collection. This identification required a highly efficient sampling system capable of quantitatively extracting odor signatures from the target source for further 
instrumental analysis. As explained in this task, SPME-GC-MS/ECD and liquid injection identified the presence of all representative volatiles within the headspace of each sample analyzed. Therefore, the explosive material analyzed was suitable for further HSCS collection. In addition, collection of pertinent blanks and the use of standards and calibrations were performed in order to assess the quality of the analytical data (shown in Appendix A).

\subsection{TASK 2: EVALUATION AND OPTIMIZATION OF FLOW RATE OF THE HSCS USING REPRESENTATIVE VOCS}

This task will evaluate the impact of HSCS sampling flow rate on the accumulation efficiency of target odorants from explosives materials. Odor accumulation onto the sorbent medium was conducted using the HSCS as the collection device. All samples were stored in silanized glass vials at room temperature for instrumental analysis and the headspace of the collection medium was carried out to profile the accumulated scent mass via SPME-GC/MS or SPME-GC/ECD. The ultimate objective of this task was to determine VOC accumulation efficiency at the three different HSCS flow rates settings (low, medium, and high) using explosive material as the odor source. Sampling time for this step was set at 30 seconds, which is the default setting for the HSCS device. 
6.2.1. 2-ethyl-1-hexanol (2E1H) and 2, 3-Dimethyl-dinitrobutane (DMNB)

\subsubsection{SPME Extraction Time Optimization}

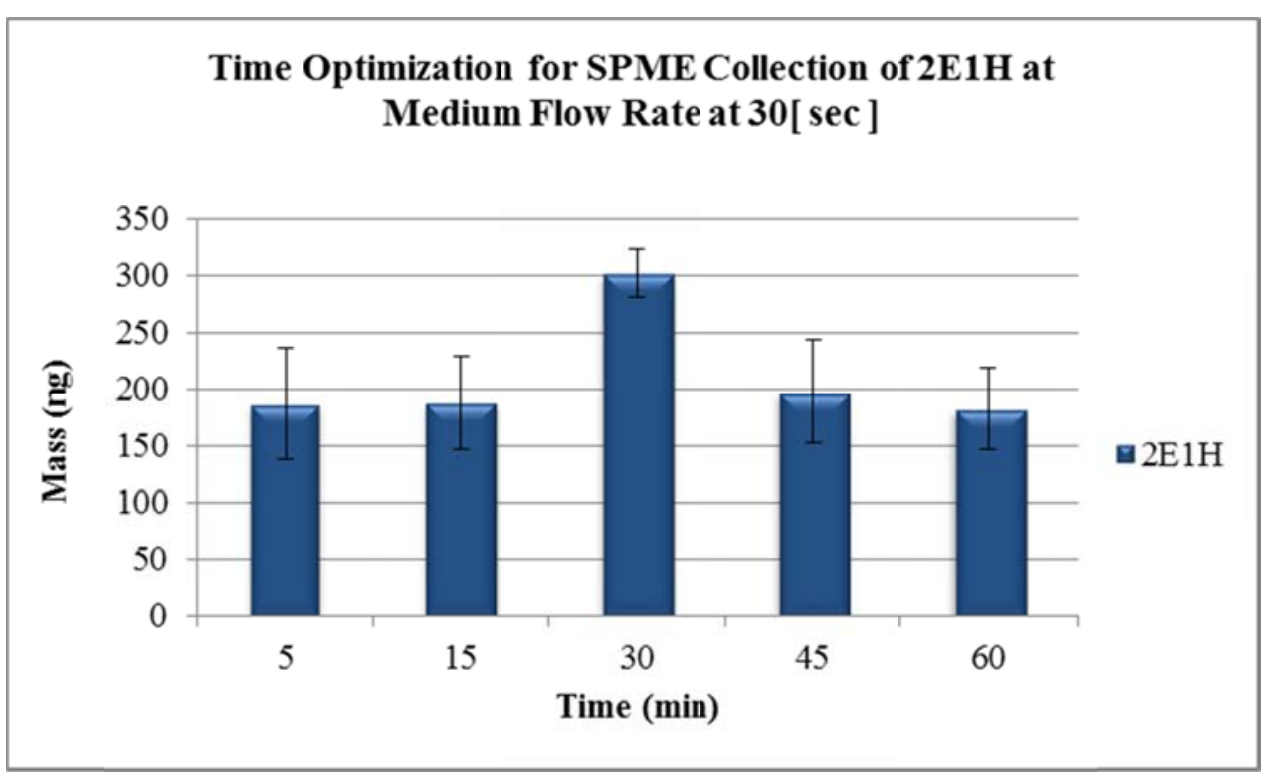

Figure 19: SPME Extraction Time Optimization of 2E1H

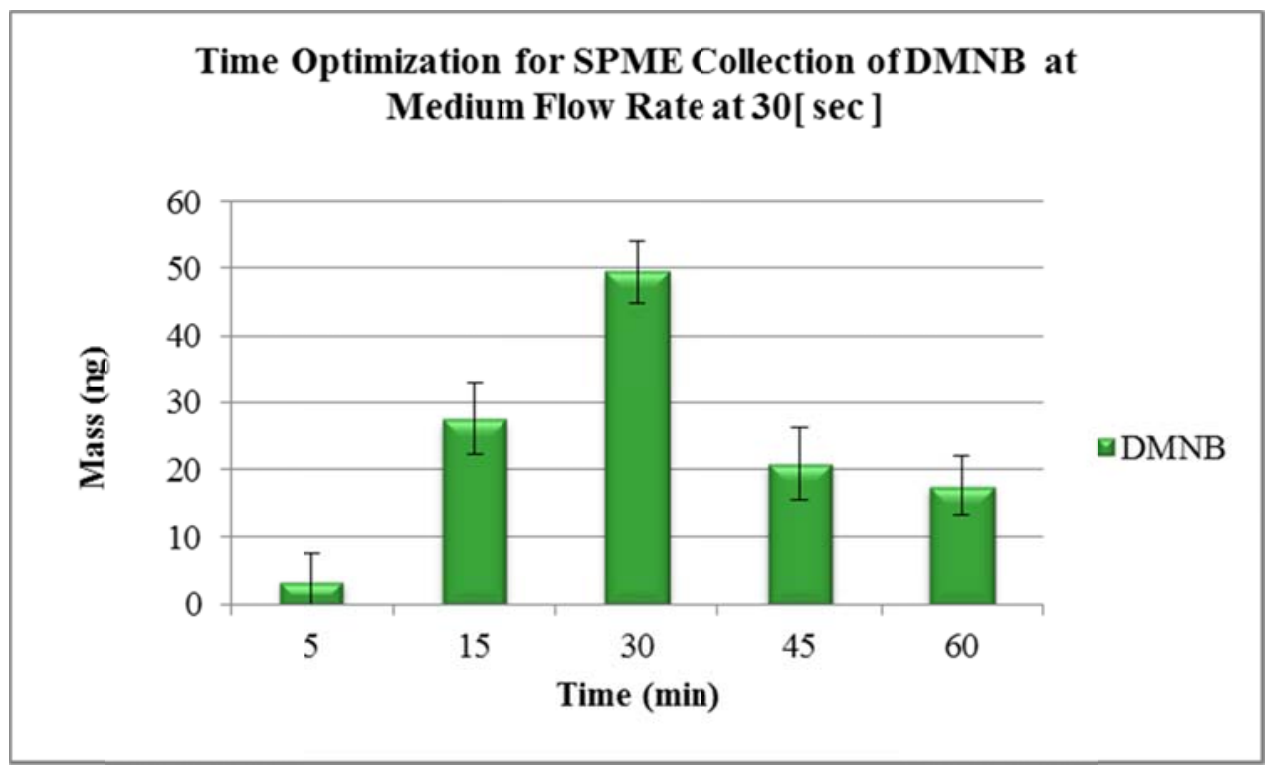

Figure 20: SPME Extraction Time Optimization of DMNB 
The results from SPME extraction are based on the peak area which is proportional to the amount of the compound that was present. To quantitate the amount of compounds being extracted by the SPME fiber, an external calibration was performed and to approximate the amount of VOCs extracted, the slope of the line obtained in the calibration curve was used as a response factor for the analyzed compound. The effect of extraction time was studied by performing a comparison of the mass obtained from each sample at the different times evaluated.

As depicted in Figure 19 and Figure 20, optimal SPME extraction time for both target odorants for this explosive category was obtained at $30 \mathrm{~min}$ in which an average mass scent of $302 \pm 22 \mathrm{ng}$ and $49.5 \pm 4.5 \mathrm{ng}$ was collected for $2 \mathrm{E} 1 \mathrm{H}$ and DMNB respectively. These samples were analyzed in a hot plate at $56{ }^{\circ} \mathrm{C}$, however; analysis of triplicate samples and blank were also made for each of the chemical compounds at room temperature for comparison purposes at the optimal SPME extraction time of $30 \mathrm{~min}$ at $56{ }^{\circ} \mathrm{C}$.

According to the results, at room temperature only a scent mass average of $103 \pm$ $10 \mathrm{ng}$ and $4.23 \pm 1.9 \mathrm{ng}$ was collected for $2 \mathrm{E} 1 \mathrm{H}$ and DMNB respectively; meaning that a greater amount of both target odorants was obtained from samples analyzed at $56{ }^{\circ} \mathrm{C}$ temperature. Overlay chromatograms of both odorants along with the correspondent standard are presented in Figure 21. 

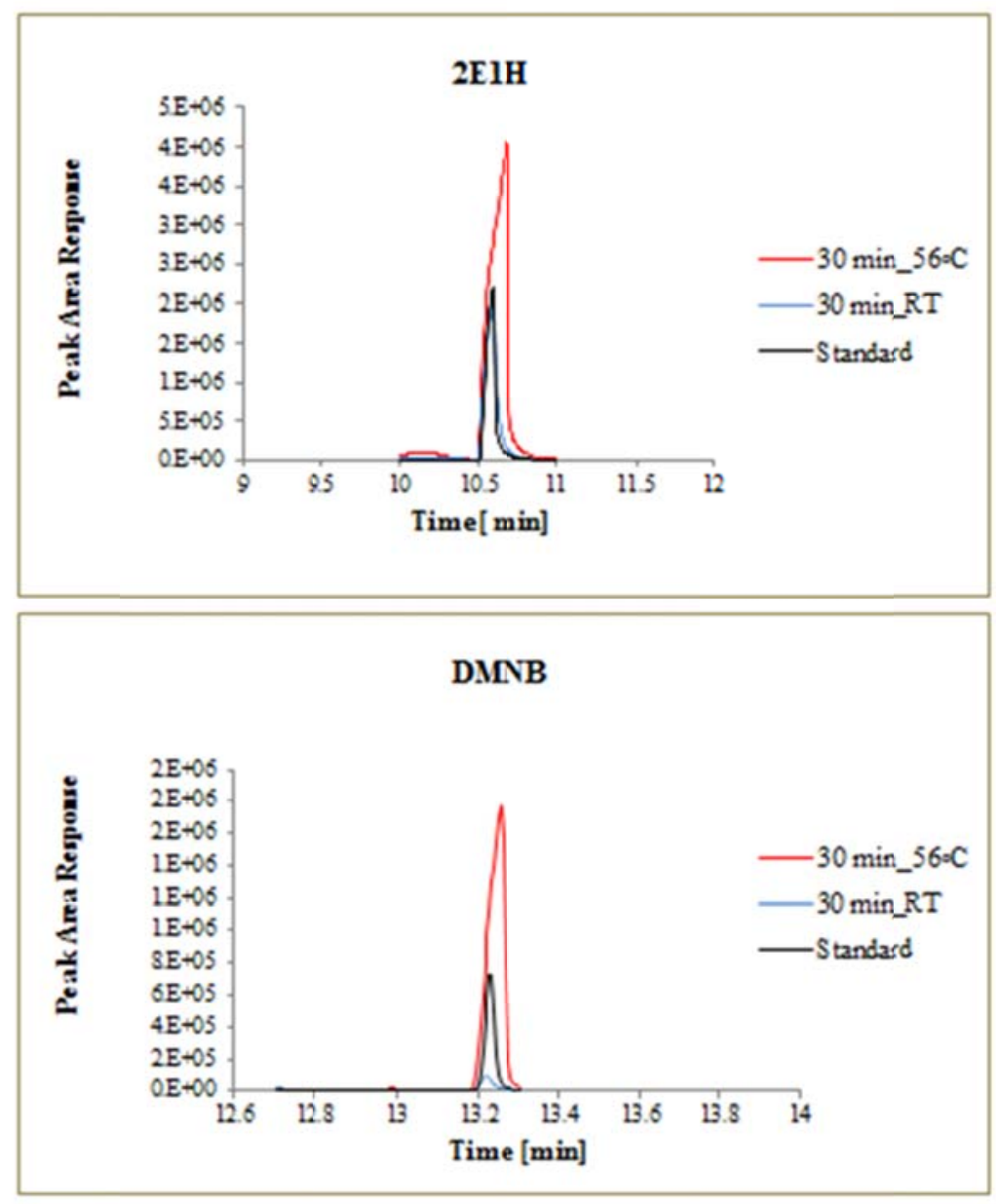

Figure 21: Comparison Chromatogram of Optimal SPME Extraction Time (30 min) of 2E1H and DMNB at Different Temperatures

\subsubsection{Flow Rate Optimization}

\section{Analysis of Samples from Miami Dade K9 Facility (source \# 2)}

The samples obtained from the Metro Dade K9 Facility (source \#2) with a mass average or $98 \mathrm{~g}$ were placed in three different crystal jars $(8 \mathrm{oz})$ for at least one (1) hr in order to obtain a homogeneous headspace distribution of the target odor. Triplicates samples were collected at atmospheric conditions $\left(25.7^{\circ} \mathrm{C}\right.$ with a relative humidity of $72.4 \%$ ) at low, medium, and high flow rates for $30 \mathrm{sec}$ by following the protocol for 
HSCS collection (section 5.3.2). The samples were stored in silanized $40 \mathrm{ml}$ glass vials after collection was completed. Immediately after collection, vials were placed in a hot block at about $56{ }^{\circ} \mathrm{C}$. SPME extraction was then conducted for 30 min followed by GC/MS analysis to obtain the headspace profile of the collected active odor.

After quantitative analysis was made, the results showed that at $30 \mathrm{sec}$ sampling time the collected average mass for $2 \mathrm{E} 1 \mathrm{H}$ was $29.9 \pm 1.4 \mathrm{ng}, 34.4 \pm 9.4 \mathrm{ng}$, and $53.5 \pm$ $6.3 \mathrm{ng}$ for low, medium and high flow rates respectively (Figure 22 and Table 6). According to these results it was determined that high flow rate was the parameter where most $2 \mathrm{E} 1 \mathrm{H}$ odorant was collected. However; reproducibility at this setting was not optimal. As a result, low flow rate for $30 \mathrm{sec}$ was considered a better flow rate for the collection of $2 \mathrm{E} 1 \mathrm{H}$. This is supported by calculating the relative standard deviation (\%RSD). As depicted in Table 6, when samples are collected using medium and high flow rates, the $\%$ RSD are the greatest ( $48 \%$ and $21 \%$ respectively). However, when low flow rate is used the $\% \mathrm{RSD}$ is less at $8 \%$. The low flow rate was therefore considered to be the most appropriate parameter since the lower relative standard deviation highlights a more reproducible amount of odorant for each individual collection.

Statistical analysis (one-way Analysis of Variance, ANOVA; $\alpha=0.05$ ) showed significant differences in the amounts of target odorant collected at all different flow rates. 


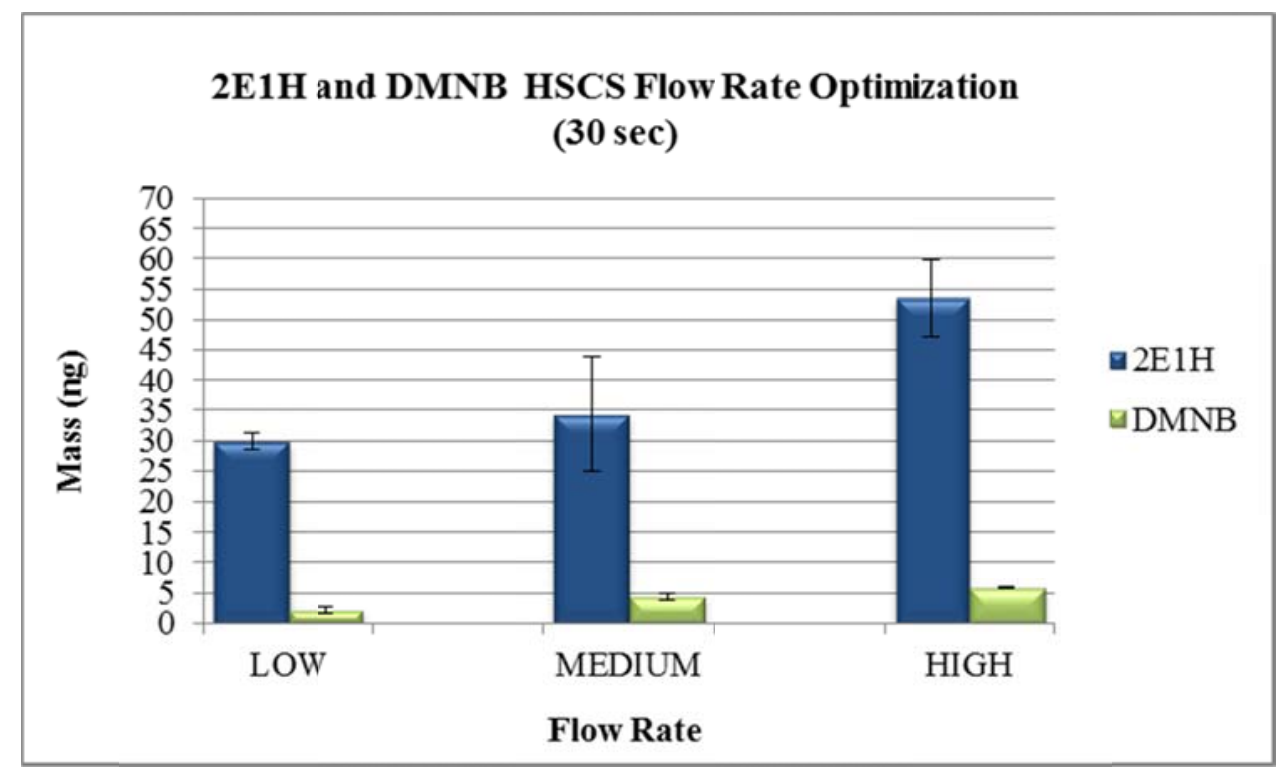

Figure 22: HSCS Collection of C4 Explosive Using Different Flow Rates at 30 sec (from Miami Dade k9 Facility)

Table 6: 2E1H Mass Average Collected form C4 Explosive at all Flow Rates at 30 $\sec (n=3)($ source \#2)

\begin{tabular}{ccc}
\hline $\begin{array}{c}\text { Flow Rate } \\
\text { (30 sec) }\end{array}$ & $\begin{array}{c}\text { Average Mass } \\
\text { Collected (ng) }\end{array}$ & RSD (\%) \\
\hline Low & $29.9 \pm 1.4$ & 8 \\
\hline Medium & $34.4 \pm 9.4$ & 48 \\
\hline High & $53.5 \pm 6.3$ & 21 \\
\hline
\end{tabular}

Table 7: DMNB Mass Average Collected form C4 Explosive at all Flow Rates at 30 $\sec (n=3)$ (source \#2)

\begin{tabular}{ccc}
\hline $\begin{array}{c}\text { Flow Rate } \\
\text { (30 sec) }\end{array}$ & $\begin{array}{c}\text { Average Mass } \\
\text { Collected (ng) }\end{array}$ & RSD (\%) \\
\hline Low & $2.25 \pm 0.6$ & 44 \\
Medium & $4.36 \pm 0.4$ & 18 \\
\hline High & $5.87 \pm 0.1$ & 4 \\
\hline
\end{tabular}


Quantitative analysis for DMNB target odorant was performed as well and the results showed that the mass collected at $30 \mathrm{sec}$ was $2.25 \pm 0.6 \mathrm{ng}$ for low, $4.36 \pm 0.4 \mathrm{ng}$ for medium, and $5.87 \pm 0.1 \mathrm{ng}$ for high flow rate respectively (Table 7).

According to these results, it was determined that high flow rate for $30 \mathrm{sec}$ was the parameter where the most DMNB odorant was collected and reproducibility at this setting was the best. As a result, high flow rate for $30 \mathrm{sec}$ was considered the optimal flow rate for the collection of DMNB. This was supported by calculating the relative standard deviation (\%RSD). As seen in Table 7, when samples are collected at low flow rate the $\% \mathrm{RSD}$ is the greatest (44\%). However, when high flow rate is used the $\% \mathrm{RSD}$ is less: $4 \%$. High flow rate was therefore considered the most appropriate parameter for the dynamic collection of DMNB odorant.

Statistical analysis (one-way Analysis of Variance, ANOVA; $\alpha=0.05$ ) showed significant differences in the amounts of target odorant collected using all flow rates for $30 \mathrm{sec}$.

\section{Analysis of Samples from the ATF in Tennessee (source \# 3)}

Samples obtained from the ATF (source \#3) with a mass average of $70 \mathrm{~g}$ were placed each in glass containers ( $8 \mathrm{oz}$ ) for HSCS collection following the same HSCS collection procedure previously explained (section 5.3.2). For the comparison of the three different flow rates, samples were collected at atmospheric conditions $\left(22.3{ }^{\circ} \mathrm{C}\right.$ with a relative humidity of $34 \%$ ). The optimal flow rate was studied by conducting a comparison of the average of mass collected for all three (3) trials at each flow rate for 30 sec as depicted in Figure 23. 


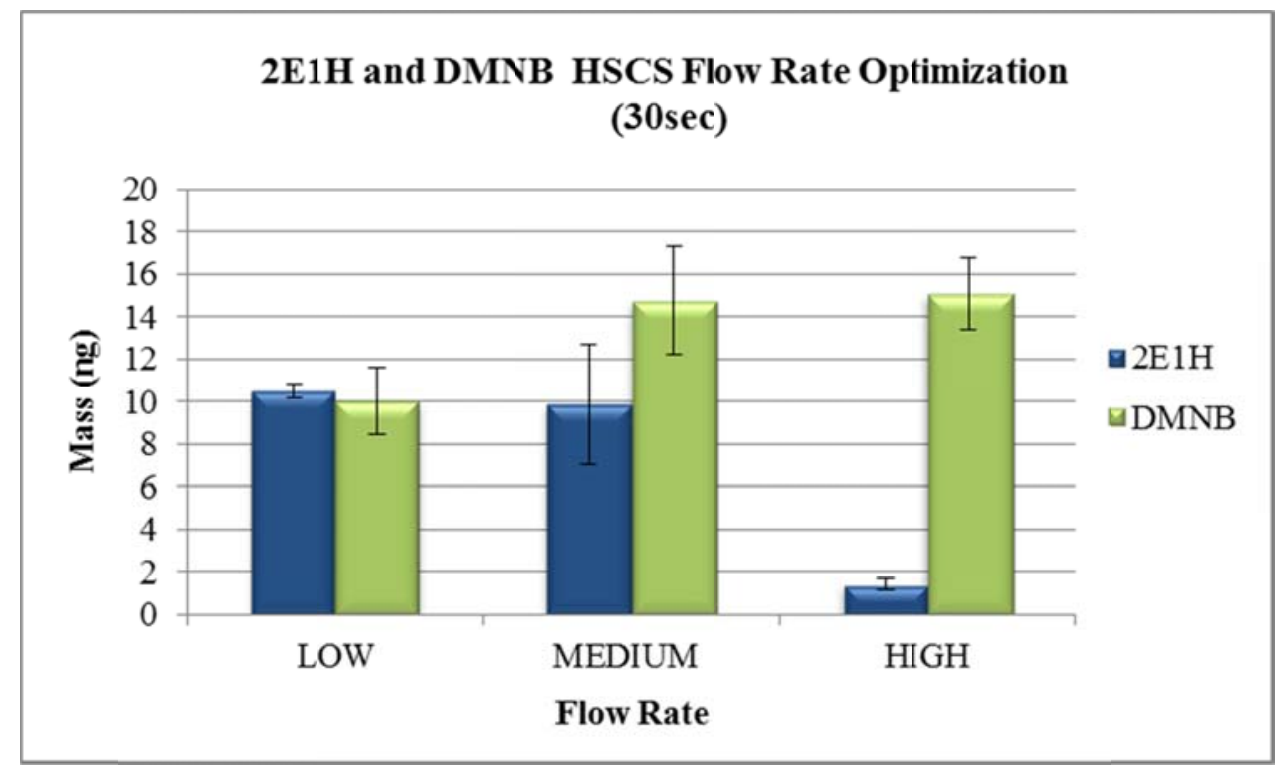

\section{Figure 23: HSCS Collection of C4 Explosive Using Different Flow Rates at 30 sec (from the ATF)}

The results showed that the mass collected at $30 \mathrm{sec}$ for $2 \mathrm{E} 1 \mathrm{H}$ was $10.5 \pm 0.3 \mathrm{ng}$ using low, $9.87 \pm 2.8 \mathrm{ng}$ for medium and $1.42 \pm 0.3 \mathrm{ng}$ for high flow rate respectively Table 8). According to these results it was determined that low flow rate for $30 \mathrm{sec}$ was the parameter where the most $2 \mathrm{E} 1 \mathrm{H}$ odorant was collected and reproducibility at this setting was the best. As a result, low flow rate for $30 \mathrm{sec}$ was considered a better flow rate for the collection of $2 \mathrm{E} 1 \mathrm{H}$. This was supported by calculating the relative standard deviation $(\% \mathrm{RSD})$. As depicted in Table 8 when samples are collected using medium flow rate at 30 seconds, the $\% \mathrm{RSD}$ is the greatest (50\%). However, when low flow rate is used the $\%$ RSD is less at $5 \%$. The low flow rate was therefore considered to be the most appropriate parameter for dynamic collection of $2 \mathrm{E} 1 \mathrm{H}$ target volatile.

Statistical analysis (one-way Analysis of Variance, ANOVA; $\alpha=0.05$ ) showed significant differences in the amounts of target odorant collected when low and medium 
were compared with high flow rate. However, there was no significant difference when low and medium rates were compared to each other.

Table 8: 2E1H Mass Average Collected from C4 Explosive at all Flow Rates at 30 $\sec (n=3)$ (source\#3)

\begin{tabular}{ccc}
\hline $\begin{array}{c}\text { Flow Rate } \\
\text { (30 sec) }\end{array}$ & $\begin{array}{c}\text { Average Mass } \\
\text { Collected (ng) }\end{array}$ & \multicolumn{1}{c}{ RSD (\%) } \\
\hline Low & $10.5 \pm 0.3$ & 5 \\
\hline Medium & $9.87 \pm 2.8$ & 50 \\
\hline High & $1.42 \pm 0.3$ & 37 \\
\hline
\end{tabular}

The results for quantitative analysis of DMNB target odorant showed that the mass collected at $30 \mathrm{sec}$ was $10.0 \pm 1.6 \mathrm{ng}$ for low, $14.8 \pm 2.5 \mathrm{ng}$ for medium and $15.1 \pm 1.7 \mathrm{ng}$ for high flow rate respectively (Table 9).

Table 9: DMNB Mass Average Collected from C4 Explosive at all Flow Rates at 30 $\sec (\mathbf{n}=3)($ source \#3)

\begin{tabular}{ccc}
\hline $\begin{array}{c}\text { Flow Rate } \\
\text { (30 sec) }\end{array}$ & $\begin{array}{c}\text { Average Mass } \\
\text { Collected (ng) }\end{array}$ & RSD (\%) \\
\hline Low & $10.0 \pm 1.6$ & 27 \\
\hline Medium & $14.8 \pm 2.5$ & 30 \\
\hline High & $15.1 \pm 1.7$ & 19 \\
\hline
\end{tabular}

The results demonstrate that high flow rate for $30 \mathrm{sec}$ was the parameter that most DMNB odorant was collected and reproducibility at this setting was the best. As a result, high flow rate for $30 \mathrm{sec}$ was considered the optimal flow rate for the collection of DMNB. This is supported by calculating the relative standard deviation (\%RSD). As 
depicted in Figure 23, when samples are collected using medium $30 \mathrm{sec}$, the \%RSD is the greatest $(30 \%)$. However, when high flow rate is used the \%RSD is less, 19 as seen in Table 9. High flow rate was therefore considered the most appropriate parameter in comparison with low and medium flow rates. Statistical analysis (one-way Analysis of Variance, ANOVA; $\alpha=0.05$ ) showed no significant differences in the amounts of target odorant collected using all flow rates for $30 \mathrm{sec}$.

\section{Comparison of HSCS Collection at 30 seconds for Both Sources}

It can be concluded that during sampling procedures, optimal flow rate at 30 seconds obtained at both sources for representative VOCs from C4 explosive was the same. Target odorant $2 \mathrm{E} 1 \mathrm{H}$ was effectively released from the $\mathrm{C} 4$ explosive material and transferred onto the gauze by utilizing the HSCS at a low flow rate. However, the greatest collection of DMNB target odorant was obtained by utilizing the HSCS at a high flow rate. A stronger flow rate was necessary in order to efficiently trap the DMNB volatile. This can be explained by the fact that DMNB has lower volatility (vapor pressure of $2.1 \mathrm{x}$ $10^{-3}$ Torr) than $2 \mathrm{E} 1 \mathrm{H}$ (vapor pressure of $1.36 \times 10^{-1}$ Torr ) ( see Table 5).

As explained in Table 10, a higher amount of $2 \mathrm{E} 1 \mathrm{H}$ was collected for samples obtained from Miami Dade k9 Facility (source \#2) while a greater amount of DMNB was collected from samples from the ATF (source \#3). 
Table 10: Comparison of 2E1H and DMNB Mass Average Collected from C4 at Optimal Flow Rate at both locations at $30 \sec (n=3)$

\begin{tabular}{ccccc}
\hline Location & Compound & $\begin{array}{c}\text { Average } \\
\text { Mass } \\
\text { Collected } \\
\text { (ng) }\end{array}$ & RSD (\%) & Flow Rate \\
\hline $\begin{array}{c}\text { Miami Dade K9 } \\
\text { Facility(source \#2) }\end{array}$ & $2 \mathrm{E} 1 \mathrm{H}$ & $29.9 \pm 1.4$ & 8 & Low \\
\hline ATF (source \#3) & DMNB & $5.87 \pm 0.1$ & 4 & High \\
& DE1H & $10.5 \pm 0.3$ & 5 & Low \\
\hline
\end{tabular}

The results obtained with these two representative odorants from $\mathrm{C} 4$ explosive material demonstrate that each target volatile behaves in a distinctive manner and that the interaction of each chemical with the collection medium (gauze matrix) as well as its intrinsic physical properties such as volatility affects the amount of odor obtained at a specific time setting and flow rate used during sample collection.

\subsubsection{2, 4-Dinitrotoluene (2, 4- DNT)}

\subsubsection{SPME Extraction Time Optimization}

The effect of extraction time was studied by performing a comparison of the mass obtained from each sample at different times (Figure 24). Upon completion of data collection it was found that $21 \mathrm{hr}$ of extraction time provided the greatest abundance of the target odorant which $1.49 \pm 0.1 \mathrm{ng}$ of $2,4-\mathrm{DNT}$ were collected. Statistical analysis was conducted (one-way Analysis of Variance, ANOVA; $\alpha=0.05$ ) and revealed that at 21 hr there was a significant difference in the amount of odorant collected when compared 
with the other time intervals. Therefore, $21 \mathrm{hr}$ of SPME analysis has been set as the optimal extraction time for collection of 2,4-DNT as the active odor from single based smokeless powder.

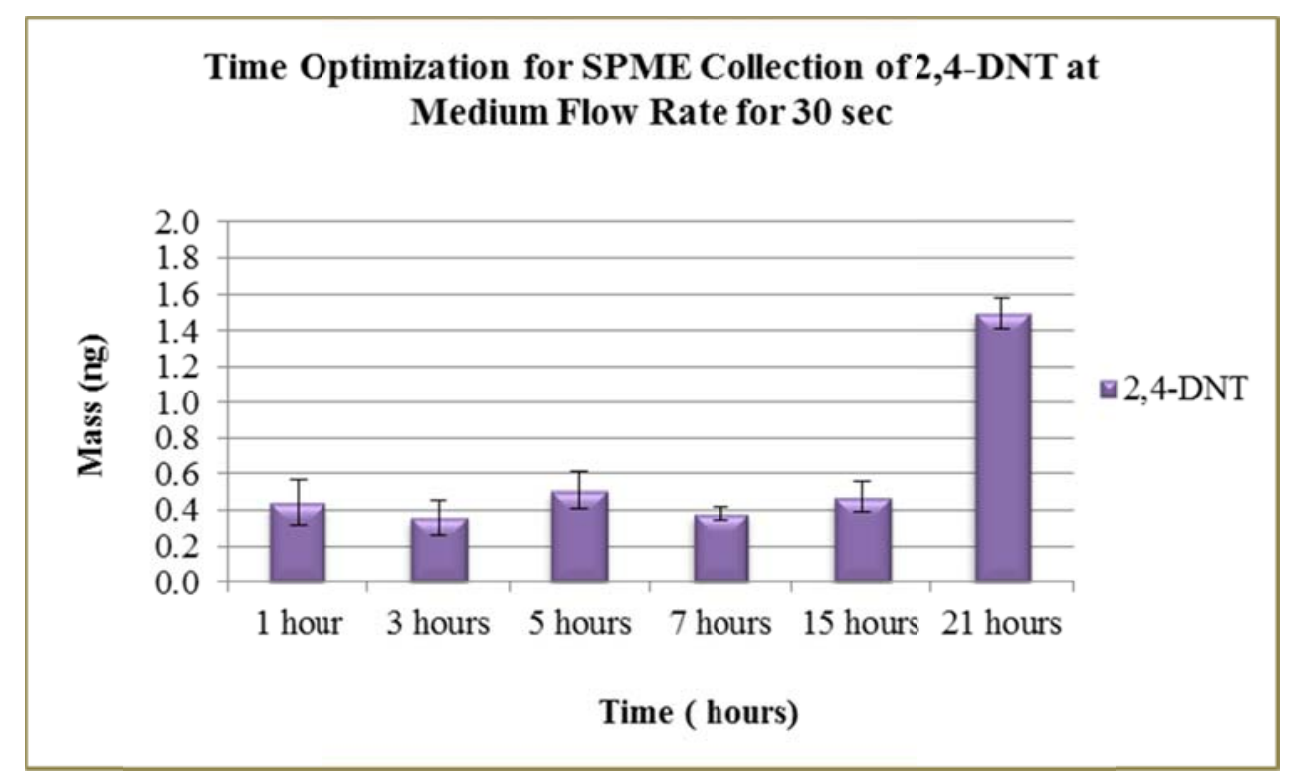

Figure 24: SPME Extraction Time Optimization of 2,4-DNT

\subsubsection{Flow Rate Optimization}

The flow rate was studied by conducting a comparison of the average mass collected of 2, 4-DNT at each flow rate for 30 seconds as shown in Figure 25. As can be observed, the extracted mass for each flow rate at 30 seconds was enough to be detected as seen in Table 11. Statistical analysis was conducted (one-way Analysis of Variance, ANOVA; $\alpha=0.05)$. Upon completion of data collection it was found that when samples are collected for 30 seconds there is no significant difference in the amount of VOC collected among the three (3) different air flow collection rates. However; after the evaluation of the different flow rates for 30 seconds, high was determined to be the most 
suitable HSCS flow rate to be used for the collection of 2,4-DNT signature odor since the relative standard deviation value (RSD\%) at this setting was the lowest: RSD\% of 48 when compared with low and medium flow rates as expressed in Table 11.

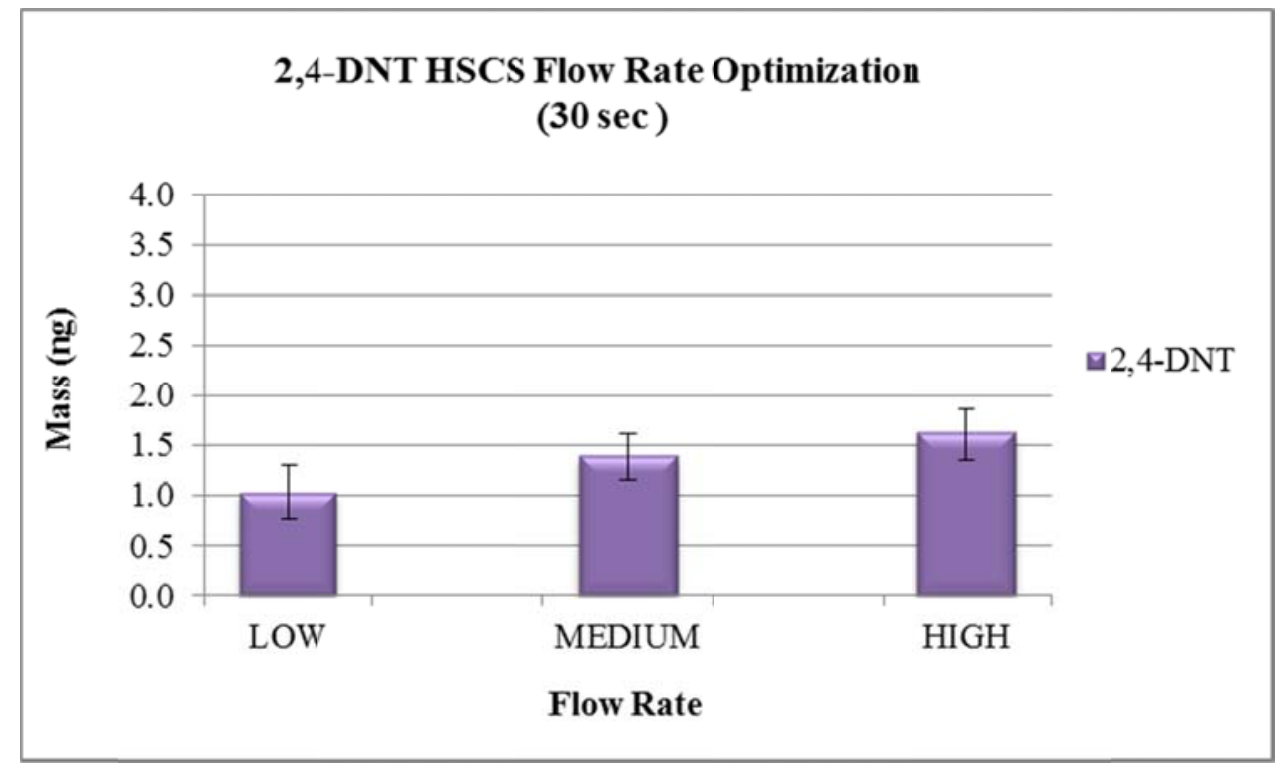

Figure 25: Optimal Flow Rate for 2,4-DNT for 30 sec Using the HSCS

Table 11: 2,4-DNT Mass Average Collected at all Flow Rates at $30 \sec (n=9)$

\begin{tabular}{ccc}
\hline Flow Rate & $\begin{array}{c}\text { Average Mass } \\
\text { Collected (ng) }\end{array}$ & RSD (\%) \\
\hline Low & $1.03 \pm 0.3$ & 77 \\
Medium & $1.38 \pm 0.2$ & 51 \\
\hline High & $1.62 \pm 0.3$ & 48 \\
\hline
\end{tabular}

\subsubsection{Flow Rate Optimization of TNT}

An alternate explosive material containing the same signature volatile 2, 4-DNT was evaluated. This analysis includes the evaluation of TNT, a secondary explosive 
material containing 2, 4-DNT. For the evaluation of this explosive the same SPMEGC/ECD method (section 5.5) for the analysis of single based smokeless powder for detection of 2,4-DNT signature odorant was followed.

The extraction of signature VOC emanating from TNT explosive material was conducted under controlled conditions $\left(20.4^{\circ} \mathrm{C}\right.$ with a relative humidity of $\left.39.0 \%\right)$ following the HSCS protocol explained in 5.3.2. Each trial was conducted on the same day with a corresponding control sample to monitor for any background/contamination issues.

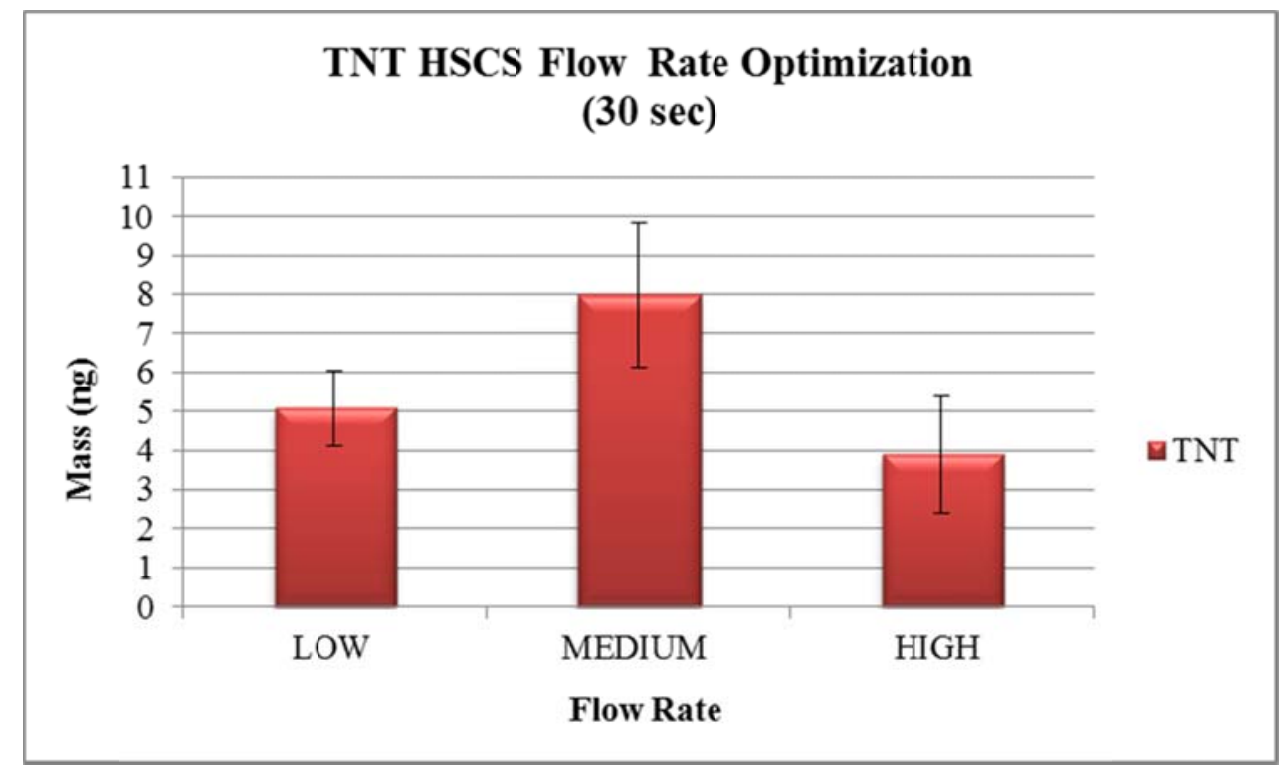

Figure 26: HSCS Collection of TNT Using Different Flow Rates at 30 seconds

2, 4 DNT odorant collected from TNT at all flow rates is shown in Figure 26. After the data was collected and analyzed, based on peak area (proportional to amount of volatile) quantitative analysis was performed and the results showed that the scent mass collected at $30 \mathrm{sec}$ was of $5.09+0.9 \mathrm{ng}$ for low, $7.98+1.8 \mathrm{ng}$ for medium, and $3.89 \pm 1.5$ ng for high flow rate respectively. According with these results it was determined that 
even though medium flow rate for 30 seconds was the parameter where most 2, 4-DNT odorant was collected, reproducibility at this setting was not optimal. As a result, low flow rate for 30 seconds was considered a better flow rate for the collection of TNT. This is supported by calculating the relative standard deviation (\%RSD). As depicted in Table 12, when samples are collected using high 30 seconds, the $\%$ RSD is the greatest at $67 \%$. However, when low flow rate is used the $\% \mathrm{RSD}$ is less at $33 \%$. The low flow rate was therefore considered to be the most appropriate parameter in comparison with low and medium flow rates.

Statistical analysis (one-way Analysis of Variance, ANOVA; $\alpha=0.05$ ) showed no significant differences in the amounts of target odorant collected using all flow rates for 30 seconds.

Table 12: 2,4-DNT Mass Average Collected form TNT at all Flow Rates for $30 \sec (n=3)$

\begin{tabular}{ccc}
\hline $\begin{array}{c}\text { Flow Rate } \\
\text { (30 sec) }\end{array}$ & $\begin{array}{c}\text { Average Mass } \\
\text { Collected (ng) }\end{array}$ & RSD (\%) \\
\hline Low & $5.09 \pm 0.9$ & 33 \\
\hline Medium & $7.98 \pm 1.8$ & 40 \\
High & $3.89 \pm 1.5$ & 67 \\
\hline
\end{tabular}

\subsubsection{Nitroglycerin (NG)}

\subsubsection{SPME Extraction Time Optimization}

Optimal SPME extraction time of NG representative volatile was determined by performing a comparison of the mass obtained from each sample at different times 
(Figure 27). Upon completion of data collection it was found that $21 \mathrm{hr}$ of extraction time provided the greatest abundance of the target odorant in which $0.37 \pm 0.1 \mathrm{ng}$ of nitroglycerin target odorant were collected.

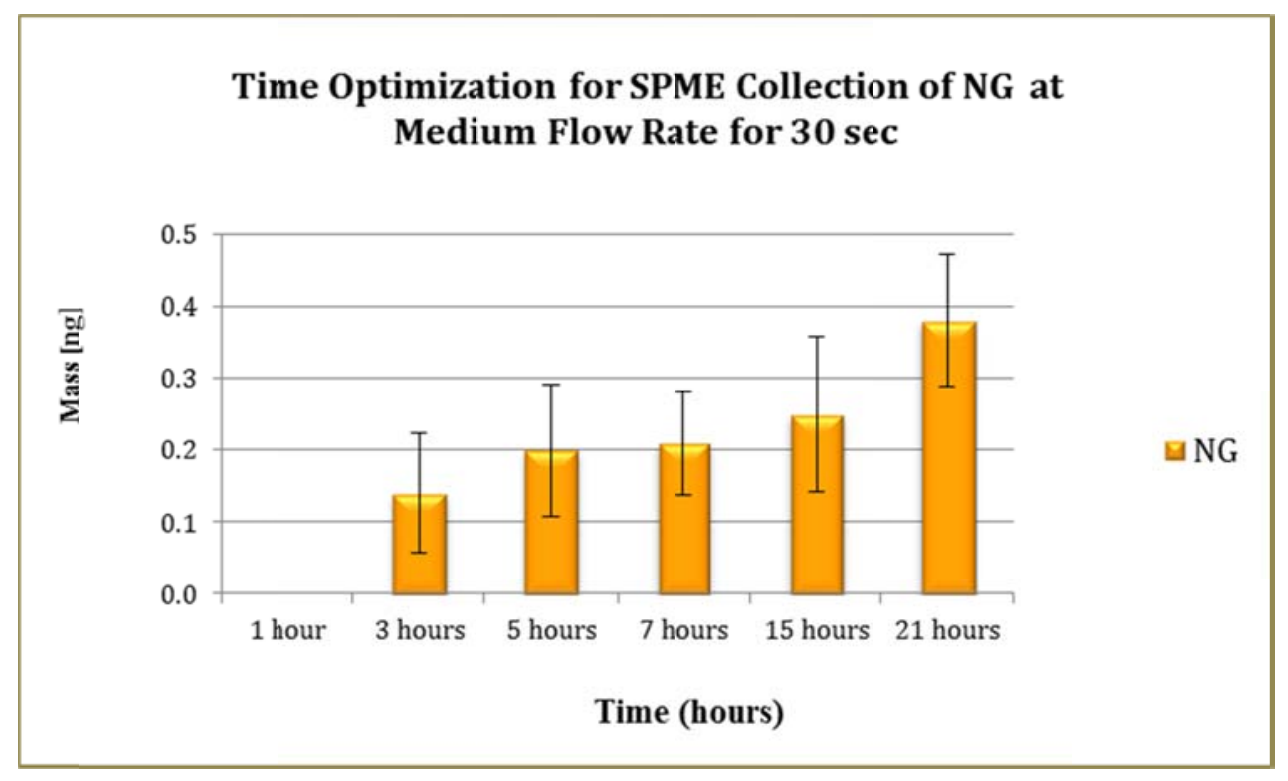

Figure 27: SPME Extraction Time Optimization of NG

Statistical analysis was conducted (one-way Analysis of Variance, ANOVA; $\alpha=0.05$ ) and revealed that at $21 \mathrm{hr}$ there was a significant difference in the amount of odorant collected when compared to the other sampling times. Therefore, $21 \mathrm{hr}$ of SPME analysis has been set as the optimal extraction time for collection of NG as the active odor from double based smokeless powder.

\subsubsection{Flow Rate Optimization}

Samples of double based smokeless powder were collected at indoor laboratory conditions $\left(21.1{ }^{\circ} \mathrm{C}\right.$ with a relative humidity of $\left.53.5 \%\right)$. The results at the low, medium, and high air flow rates of these nine (9) samples containing the active signature odor 
nitroglycerin (NG) are summarized in Figure 28. The average mass collected was $0.12 \pm$ $0.1 \mathrm{ng}, 0.21 \pm 0.1 \mathrm{ng}$, and $0.61 \pm 0.1 \mathrm{ng}$ for low, medium, and high flow rates respectively (see Table 13).

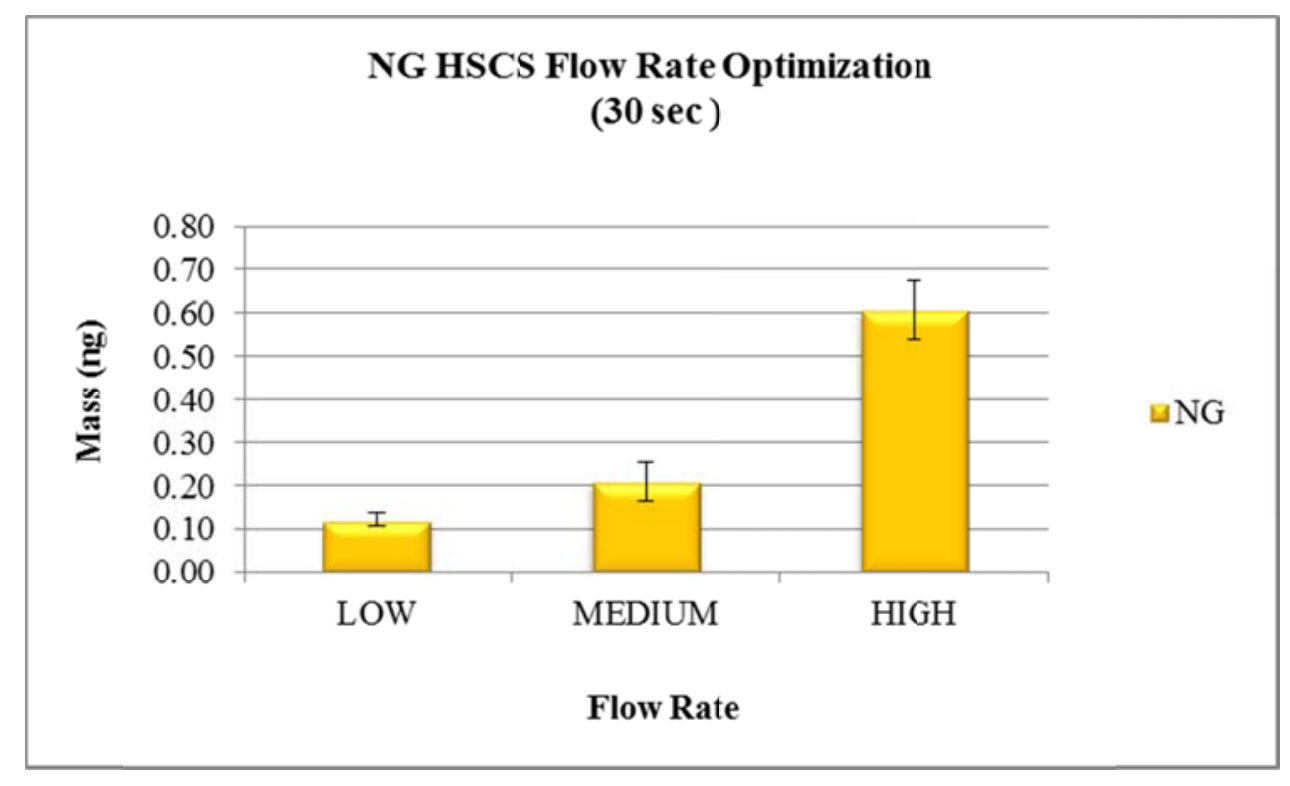

Figure 28: HSCS Flow Rate Optimization of Nitroglycerin at 30 sec

Table 13: NG Mass Average Collected at all Flow Rates for $30 \sec (n=9)$

\begin{tabular}{ccc}
\hline Flow Rate & $\begin{array}{c}\text { Average Mass } \\
\text { Collected (ng) }\end{array}$ & RSD (\%) \\
\hline Low & $0.12 \pm 0.1$ & 38 \\
Medium & $0.21 \pm 0.1$ & 60 \\
\hline High & $0.61 \pm 0.1$ & 33 \\
\hline
\end{tabular}

According to these results, it was determined that high flow rate for $30 \mathrm{sec}$ was the parameter where the greatest amount of NG was collected and the reproducibility at this setting was the best. This was supported by calculating the relative standard deviation 
(\%RSD). As depicted in Table 13, when samples are collected using high flow rate for $30 \mathrm{sec}$, the \%RSD is the lowest at 33. Statistical analysis (one-way Analysis of Variance, ANOVA; $\alpha=0.05$ ) showed there is a significant difference in the amounts of target odorant collected using all flow rates for $30 \mathrm{sec}$. The optimal parameter was selected based on the most reproducible results obtained (lowest \%RSD). Therefore, high flow rate at $30 \mathrm{sec}$ was considered to be the most appropriate parameter for dynamic collection of NG when compared with low and medium flow rates.

\subsubsection{Conclusions}

The capability of the HSCS to effectively trap and consequently release the target explosive odors has been demonstrated in this task. These results could very well highlight the influence of breakthrough effects during sampling procedures. For DMNB odorant from C4, NG and 2, 4 DNT volatiles from single and double based smokeless powder respectively; the faster airflow volume passing through the HSCS opening is required for the volatile to be effectively trapped onto the gauze medium. On the other hand, 2E1H volatile from $\mathrm{C} 4$ and 2, $4 \mathrm{DNT}$ from TNT the lower airflow speed causes the volatiles to move from the explosive material onto the gauze medium with minor headspace loss. 


\subsection{TASK 3: EVALUATION AND OPTIMIZATION OF SAMPLING TIME (30 SEC V. 60 SEC) OF REPRESENTATIVE VOCS ACCUMULATION USING THE HSCS}

This task evaluated the impact of HSCS time of sampling on the accumulation efficiency of odors from explosives materials. The available collection times offered by the HSCS device are 30 and 60 seconds. For this reason, a comparison of collection of VOCs at 30 seconds with 60 seconds was performed in order to determine the optimal HSCS sampling time of VOCs collection from the explosive materials. The ultimate objective of this task was to optimize the sampling time for the collection of explosive VOCs. The flow rate of the HSCS in this task was in accordance with the previously determined optimal flow rate in Task 2 for each corresponding explosive category.

\subsubsection{Sampling Time of $2 E 1 H$ and DMNB Representative Volatiles}

HSCS collection of samples and SPME extraction was performed according to procedures explained in sections 5.3.2 and 5.6. In order to determine the optimal flow rate and sampling time for both target odorants (2E1H and DMNB) from C4 explosive material, the best flow rate at 30 seconds for each of the volatiles (low for $2 \mathrm{E} 1 \mathrm{H}$ and high flow rate for DMNB determined in previous task) was compared with 60 seconds sampling time (Figure 29).

Since both odorants $2 \mathrm{E} 1 \mathrm{H}$ and $\mathrm{DMNB}$ are present within the $\mathrm{C} 4$ explosive material, a flow rate that provided similar and fair reproducibility for both signature

odorants was chosen. As depicted in Table 14, high flow rate at 60 seconds showed a very similar \%RSD values for each volatile; $28 \%$ and $20 \%$ for $2 \mathrm{E} 1 \mathrm{H}$ and DMNB 
respectively (highlighted in red). In contrast, when samples are collected using low flow rate for 60 seconds the \%RSD values obtained for both are significantly different from each other. For this reason; high flow rate for 60 seconds was determined to be the optimal for collection of $2 \mathrm{E} 1 \mathrm{H}$ and DMNB signature odorants from $\mathrm{C} 4$ explosive material, were \%RSD values are convenient for both compounds.

Table 14: Comparison of Optimal Flow rates for 2E1H and DMNB Odorants at $60 \sec (n=3)$

\begin{tabular}{|c|c|c|c|}
\hline $\begin{array}{c}\text { Flow Rate } \\
\text { (60sec) }\end{array}$ & Compound & $\begin{array}{c}\text { Average Mass } \\
\text { Collected (ng) }\end{array}$ & \%RSD \\
\hline \multirow{2}{*}{ LOW } & $2 \mathrm{E} 1 \mathrm{H}$ & $22.2+5.4$ & 42 \\
\cline { 2 - 4 } & $\mathrm{DMNB}$ & $4.91 \pm 0.7$ & 26 \\
\hline \multirow{2}{*}{$\mathrm{HIGH}$} & $2 \mathrm{E} 1 \mathrm{H}$ & $35.4 \pm 5.8$ & 28 \\
\cline { 2 - 4 } & $\mathrm{DMNB}$ & $7.13 \pm 0.8$ & 20 \\
\hline
\end{tabular}

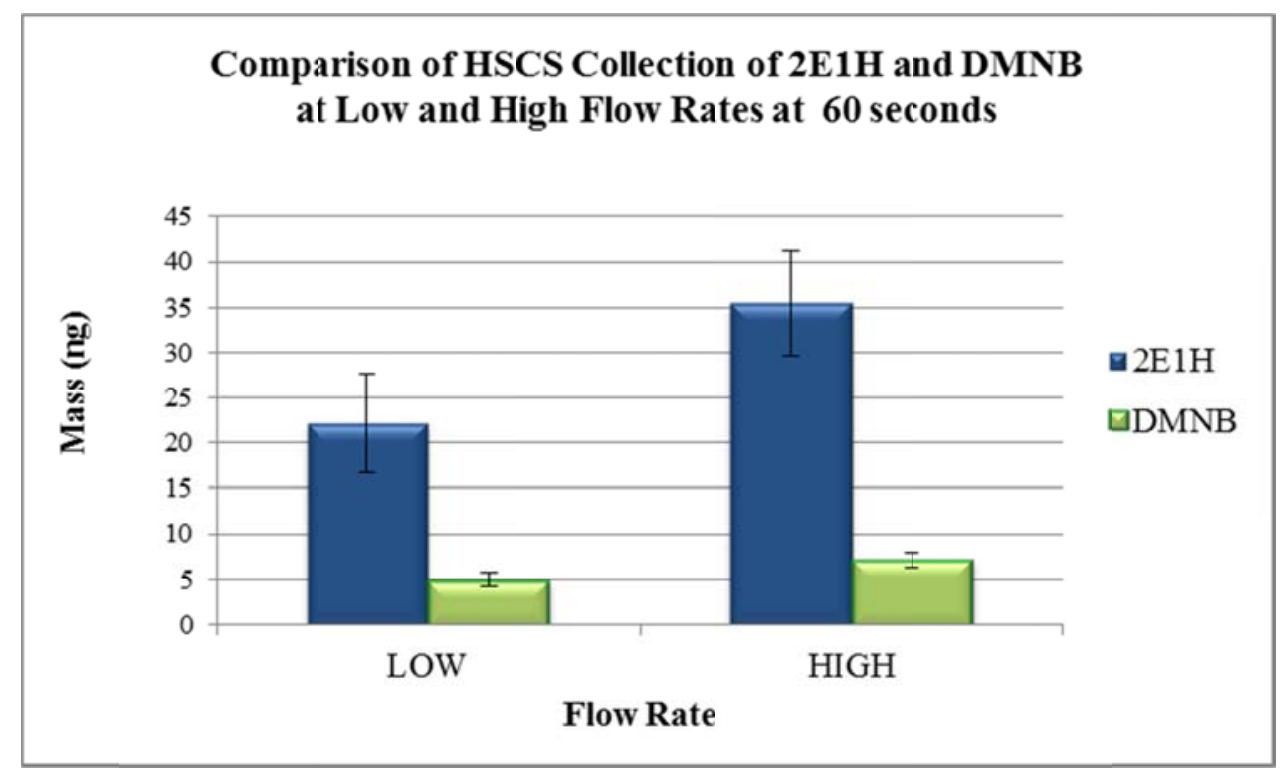

Figure 29: HSCS Collection of C4 Explosive at Low and High Flow Rates at 60 sec 


\subsubsection{HSCS Collection of C4 Explosive Material at Optimal Parameters}

Analysis of Trial Samples Obtained from City of Miami Police (source \#1)

After optimal flow rate and sampling time for HSCS collection of $2 \mathrm{E} 1 \mathrm{H}$ and DMNB was established, HSCS sampling of C4 explosive material (average mass of 157 g) was consequently performed. Three trials of triplicate samples (total of nine samples) and blanks were collected indoors on different days at about $65.7 \%$ relative humidity and temperature of $24.0{ }^{\circ} \mathrm{C}$. After the conditioning time, samples of $\mathrm{C} 4$ explosive material and blanks were collected at high flow rate at $60 \mathrm{sec}$ following the HSCS protocol explained in sections 5.3.2. After collection, cotton gauze pads were stored in silanized $40 \mathrm{~mL}$ glass vials. As soon as samples arrived to the lab, they were injected via SPME for 30 min in a hot plate at about $56{ }^{\circ} \mathrm{C}$ and eventually analyzed by $\mathrm{GC} / \mathrm{MS}$ in order to characterize the accumulated odor.

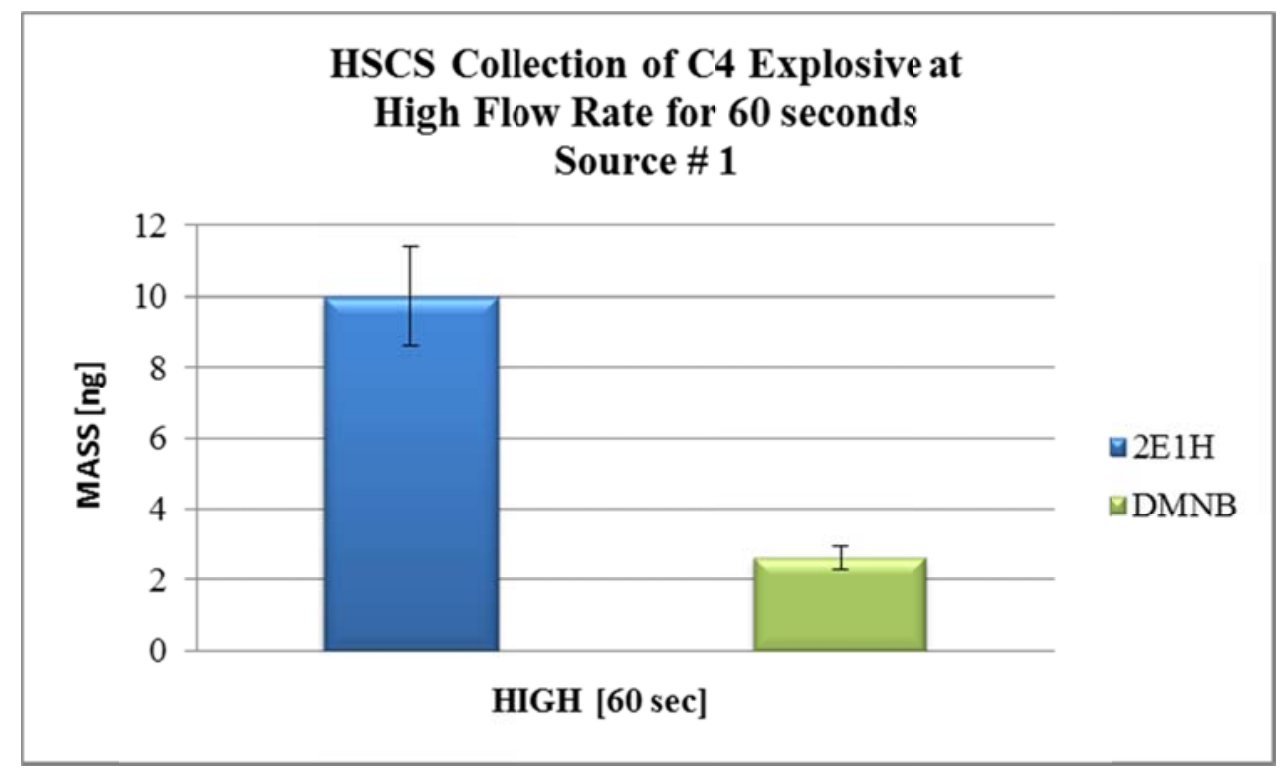

Figure 30: Semi-quantitative Analysis of C4 Volatiles (from source \#1) 
As can be observed in Figure 30, when using a high air flow rate for $60 \mathrm{sec}$ an average scent mass of $10.1 \pm 1.4 \mathrm{ng}$ (see Table 15) was collected for $2 \mathrm{E} 1 \mathrm{H}$ and $2.65 \pm 0.3 \mathrm{ng}$ for DMNB.

Table 15: C4 Explosive Mass Average Collected at High Air Flow Rate at 60 sec $(\mathbf{n}=9)$ (source \#1)

\begin{tabular}{ccc}
\hline Compound & $\begin{array}{c}\text { Average Mass } \\
\text { Collected (ng) }\end{array}$ & RSD \% \\
\hline 2E1H & $10.1 \pm 1.4$ & 41 \\
\hline DMNB & $2.65 \pm 0.3$ & 37 \\
\hline
\end{tabular}

\section{Analysis of Trial Samples Obtained from Miami Dade k9 Facility (source \#2)}

The samples obtained from source \#2 with an average mass of $98 \mathrm{~g}$ were placed each in three different glass containers $(8 \mathrm{oz})$ for HSCS collection at high flow rate for 60 sec. Same protocol as collection of samples from Source \#1 (explained above) was followed to ensure the presence of the representative explosive signature odorants. Three trials of triplicate samples (total of nine samples) and blanks were collected indoors at about $25.7^{\circ} \mathrm{C}$ with a relative humidity of $72.4 \%$.

As can be observed in Figure 31, when using a high air flow rate for $60 \mathrm{sec}$ an average scent mass of $28.60 \pm 3.7 \mathrm{ng}$ (see Table 16) was collected for $2 \mathrm{E} 1 \mathrm{H}$ and $4.78 \pm 0.8$ ng for DMNB. As depicted in Figure 29, Figure 30, and Figure 31; the target volatile 2E1H was detected at a greater amount when compared to DMNB. This can be attributed to breakthrough effects during collection as well as difficulty of volatile compound formation during conditioning period since DMNB is less volatile than 2E1H ( Table 5). 


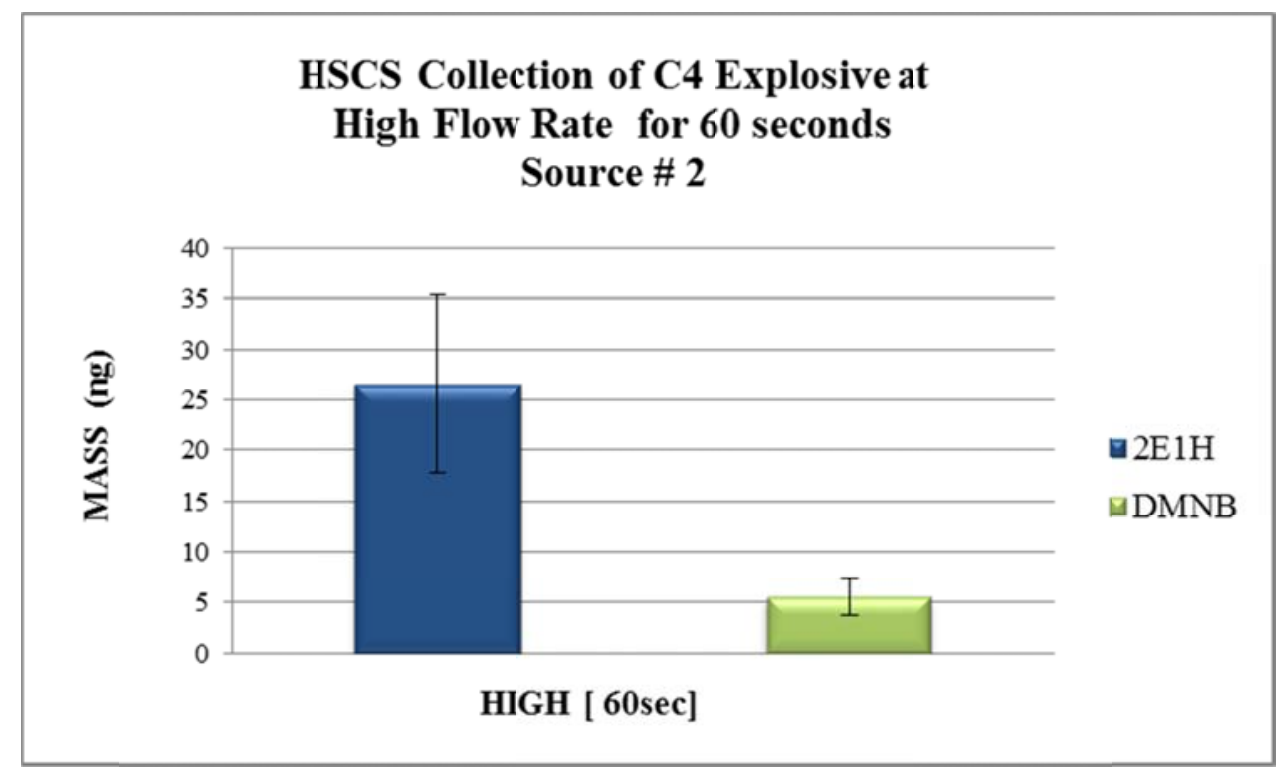

Figure 31: Semi-quantitative Analysis of C4 Volatiles (from source \#2)

Table 16: C4 Explosive Mass Average Collected at High Air Flow Rate at 60 sec $(\mathrm{n}=9)$ (source \# 2)

\begin{tabular}{crc}
\hline Compound & $\begin{array}{c}\text { Average Mass } \\
\text { Collected (ng) }\end{array}$ & RSD \% \\
\hline 2E1H & $28.6 \pm 3.7$ & 39 \\
DMNB & $4.78 \pm 0.8$ & 42 \\
\hline
\end{tabular}

\section{Analysis of Trial Samples Obtained from the ATF (source \#3)}

The same protocol for collection at $30 \mathrm{sec}$ was followed and a total of three (3) samples were taken. Each trial was conducted on the same day with a corresponding control sample to monitor for any background/contamination issues. The samples were collected at atmospheric conditions $\left(22.3{ }^{\circ} \mathrm{C}\right.$ with a relative humidity of $\left.33.7 \%\right)$.

HSCS collection of 2E1H and DMNB target odorants at optimal flow rate and sampling time: high flow at $60 \mathrm{sec}$ was performed (as seen in Figure 32). According to the results, 
the mass of VOCs collected at high flow rate for $60 \mathrm{sec}$ was $10.9 \pm 1.1 \mathrm{ng}$ for $2 \mathrm{E} 1 \mathrm{H}$ and $9.73 \pm 0.6 \mathrm{ng}$ for DMNB with a \% RSD of 18 and 11 for each volatile respectively as expressed in Table 17. It can be said that the collection of both target odorants at high flow rate for 60 seconds collected similar amounts as well as produced reproducible values.

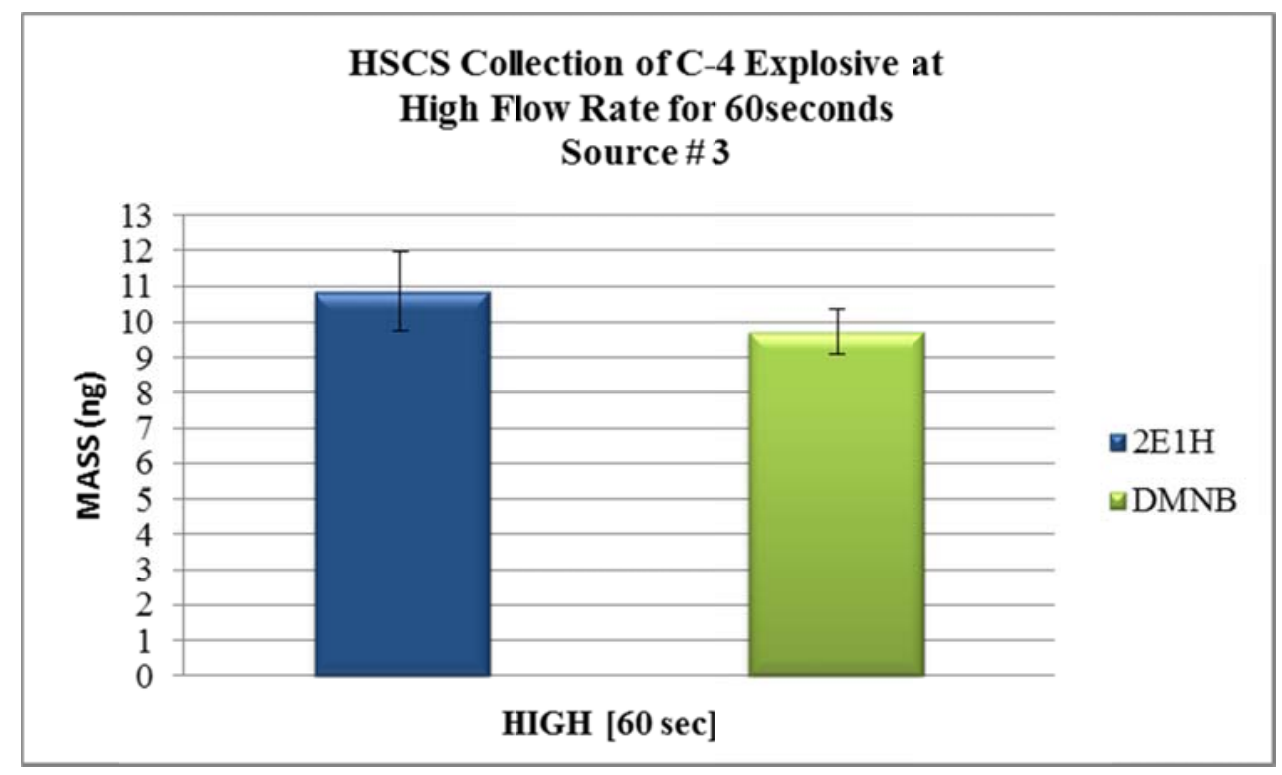

Figure 32: Semi-quantitative Analysis of C4 Volatiles (from source \#3)

Table 17: C4 Explosive Mass Average Collected at High Air Flow Rate at 60 sec $(\mathbf{n}=3)$ Source \# 3

\begin{tabular}{crc}
\hline Compound & $\begin{array}{r}\text { Average Mass } \\
\text { Collected (ng) }\end{array}$ & RSD \% \\
\hline 2E1H & $10.9 \pm 1.1$ & 18 \\
DMNB & $9.73 \pm 0.6$ & 11 \\
\hline
\end{tabular}




\section{Comparison of HSCS Collection of Representative VOCs from all Sources}

Only one set of triplicates was used for comparison of samples obtained from all three sources: City of Miami Police, the Metro Dade Canine Facility, and ATF in Tennessee as explain in section 5.6.

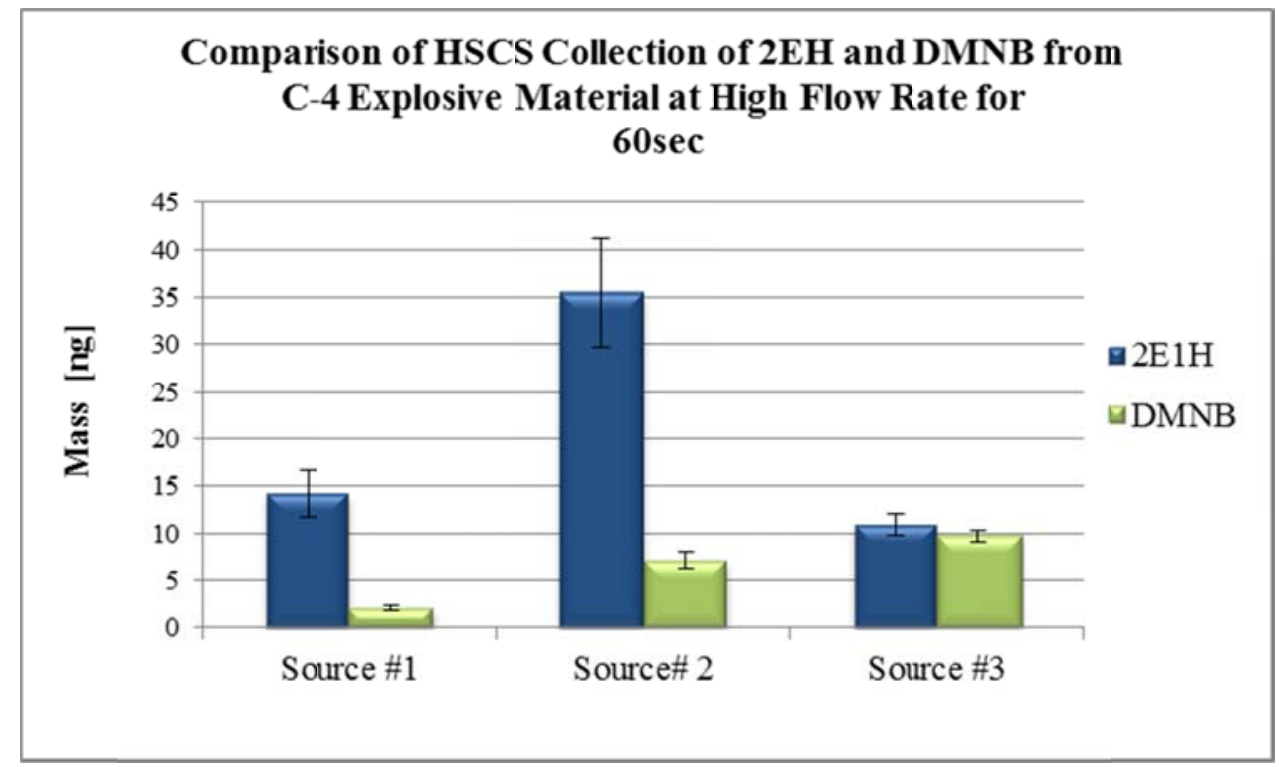

Figure 33: Comparison of HSCS Collection of VOCs from C4 Explosive from all Locations at High Flow Rate for 60 sec

Table 18: Comparison of 2E1H and DMNB Mass Average Collected from $\mathrm{C} 4$ at all Locations at High flow Rate at $60 \sec (n=3)$

\begin{tabular}{cccc}
\hline Location & Compound & $\begin{array}{c}\text { Average Mass } \\
\text { Collected (ng) }\end{array}$ & RSD (\%) \\
\hline Source \#1 & 2E1H & $14.2 \pm 2.4$ & 30 \\
& DMNB & $2.07 \pm 0.2$ & 22 \\
Source \#2 & 2E1H & $35.4 \pm 5.8$ & 28 \\
& DMNB & $7.13 \pm 0.8$ & 20 \\
\hline \multirow{2}{*}{ Source \#3 } & 2E1H & $10.9 \pm 1.1$ & 18 \\
& DMNB & $9.73 \pm 0.6$ & 11 \\
\hline
\end{tabular}


As depicted in Figure 33, collection of triplicate samples obtained from ATF (source \# 3) at high flow rate for $60 \mathrm{sec}$ showed to have collected similar amounts of both target odorants as well as to have produced fair reproducibility values when compared with collection made in Miami at source \#1 and \#2, in which samples were collected at atmospheric conditions $24.0{ }^{\circ} \mathrm{C}$ temperature and relative humidity of $65.7 \%$, and $25.7{ }^{\circ} \mathrm{C}$ with a relative humidity of $72.4 \%$ respectively. The average mass of VOCs collected at this time was significantly higher for $2 \mathrm{E} 1 \mathrm{H}$ in which double the amount was obtained: $35.4 \pm 5.8 \mathrm{ng}$ for source \# 2 versus $14.2 \pm 2.4 \mathrm{ng}$ for source \#1 and $10.9 \pm 1.1 \mathrm{ng}$ for source \#3. However; collection of DMNB volatile was similar for sources \#2 and \#3 while results from source \#1 collected less amount of the target volatile as depicted in Table 18.

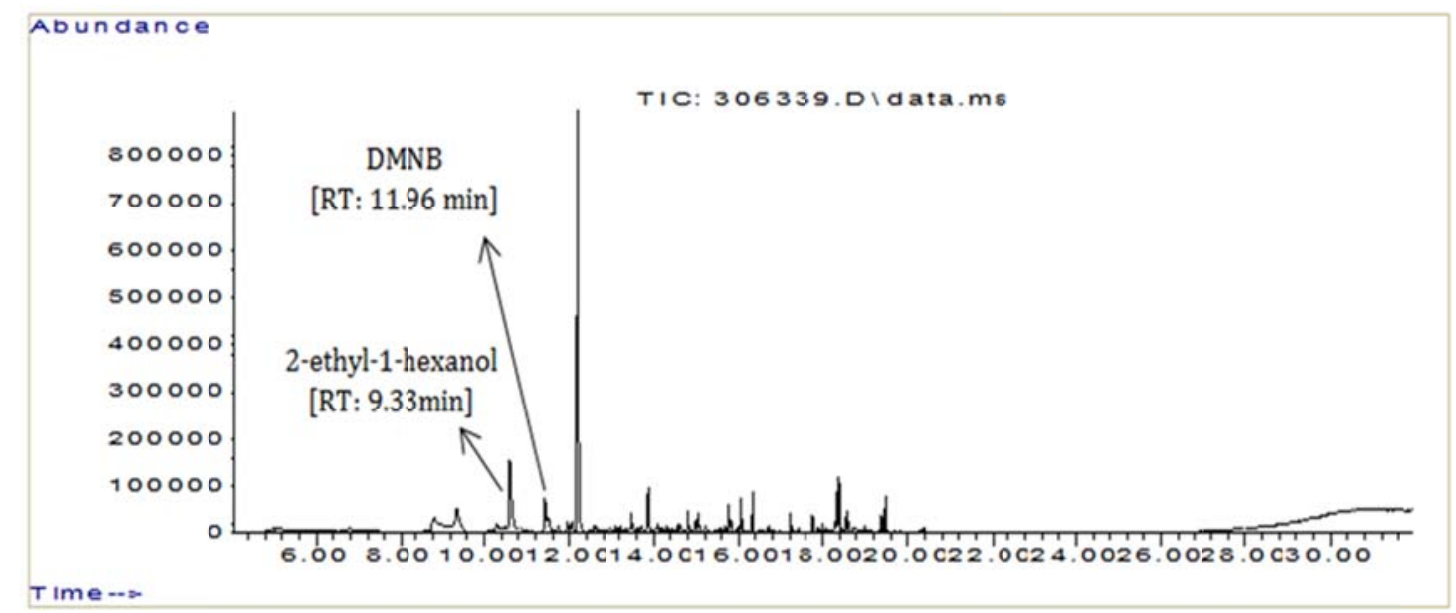

Figure 34: Headspace SPME-GC/MS of C4 Explosive Collected at High Flow Rate at $60 \mathrm{sec}$

As an example, Figure 34 presents a chromatogram of one sample from source \#1 collected at optimal high flow rate for 60 seconds showing that both target odorants are 
clearly differentiated in which $7.14 \mathrm{ng}$ and $1.63 \mathrm{ng}$ of mass scent was collected for $2 \mathrm{E} 1 \mathrm{H}$ and DMNB respectively.

The difference in mass of representative volatiles $2 \mathrm{E} 1 \mathrm{H}$ and DMNB collected at all locations can be explained by the difference in the concentration of both target odorants within the $\mathrm{C} 4$ explosive material provided by different sources. $\mathrm{C} 4$ explosive is made of cyclonite or cyclotrimethylene trinitramine (RDX) explosive, which is approximately $91 \%$ of $\mathrm{C} 4$ by weight. The other $9 \%$ is made of additives [9]. One example is $2 \mathrm{E} 1 \mathrm{H}$, a plastic binder which can vary from one manufacturer to another and the marker or taggant DMNB which is used to detect the explosive and identify its source. C4 is then the result of the combination of these ingredients in a solvent which eventually is evaporated, dried, and filtered to obtain the final product. This combination of ingredients can be mixed in different ratios by manufacturers. This explains why target odorant $2 \mathrm{E} 1 \mathrm{H}$ and DMNB will vary from source to source since specific concentrations of both compounds are not given. However, the variation of specific amounts of trapped VOCs does not necessarily equate to a negative aspect for feasible canine use. The utility of the dynamic collection system to trap target VOCs from different sources and/or settings is thereby demonstrated.

\subsubsection{Sampling Time of 2, 4-DNT Representative Volatile}

In this section of the study, same samples of Hogdon 4896 smokeless powder utilized for flow rate optimization (average mass of $25 \mathrm{~g}$ ) were used along with a correspondent blank for dynamic airflow collection at high flow rate for 60 seconds for 
comparison with the collection of the volatile 2, 4-DNT for 30 seconds flow time. Samples were collected at indoor laboratory conditions $\left(21.8^{\circ} \mathrm{C}\right.$ with a relative humidity of 53.6\%) and same protocol was followed for the collection at $30 \mathrm{sec}$ for the three (3) sets of triplicate samples (nine (9) samples) in which trials were taken at different days.

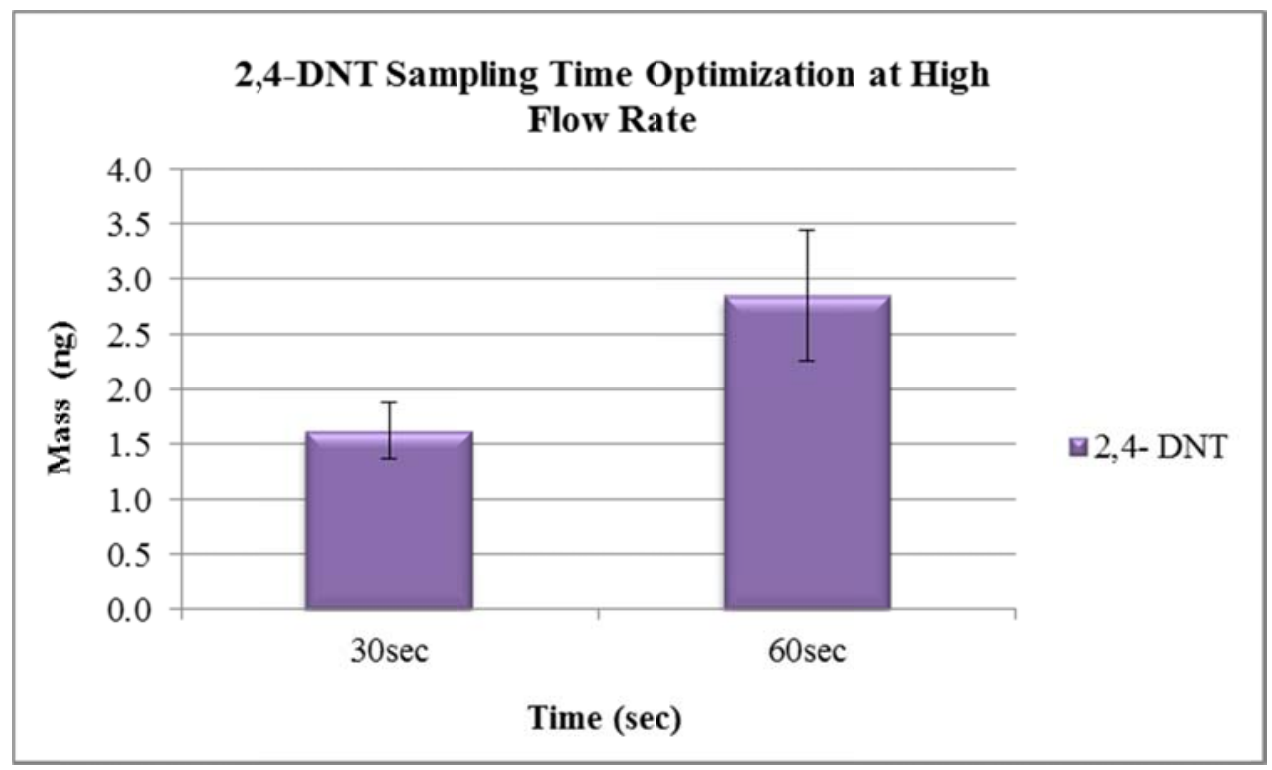

Figure 35: 2, 4- DNT Sampling Time Optimization at High Flow Rate

According to the experiment, the collection of 2, 4-DNT at high flow rate for 60 sec showed to have collected more of the target odorant. This can be attributed to the fact that at longer time, there is a better chance of trapping a greater amount of the odor within the gauze absorbent material as depicted in Figure 35. An average extracted mass of $2.85 \pm 0.59 \mathrm{ng}$ of 2, 4-DNT odorant was collected. However; reproducibility at this setting was not the optimal. This was supported by calculating the relative standard deviation (\%RSD) as seen in Table 19. On the other hand; when samples are collected at $30 \mathrm{sec}$, the $\% \mathrm{RSD}$ is the lowest at 48 . For this reason; high flow rate for $30 \mathrm{sec}$ is 
considered the most appropriate parameter compare with others since reproducibility was the best.

Table 19: 2, 4-DNT Mass Average Collected at High Flow Rate (n =9)

\begin{tabular}{ccc}
\hline $\begin{array}{c}\text { TIME } \\
\text { (sec) }\end{array}$ & $\begin{array}{c}\text { Average Mass } \\
\text { Collected (ng) }\end{array}$ & \multicolumn{1}{c}{ RSD (\%) } \\
\hline $\mathbf{3 0}$ & $1.62 \pm 0.3$ & 48 \\
\hline $\mathbf{6 0}$ & $2.85 \pm 0.6$ & 62 \\
\hline
\end{tabular}

As depicted in Table 19, the HSCS has proven to be effective in the dynamic collection of 2, 4-DNT signature odorant present in single based smokeless powder. Statistical analysis was conducted (one-way Analysis of Variance, ANOVA; $\alpha=0.05$ ) and upon completion of data collection it was found that between 30 and $60 \mathrm{sec}$ there is no significant difference in the amount of VOC collected using high air flow collection rate. Even though the statistical analysis does not highlight a significant difference between the time settings evaluated, based on the reproducibility of scent mass collected at high flow rate at $30 \mathrm{sec}$ sampling time, these parameters are recommended for an enhanced explosive mass collection.

Figure 36 shows a chromatogram of one sample of single based smokeless powder collected at the established optimal HSCS parameters of high flow rate at $30 \mathrm{sec}$ in which $1.63 \mathrm{ng}$ of 2, 4 DNT scent mass was collected. 


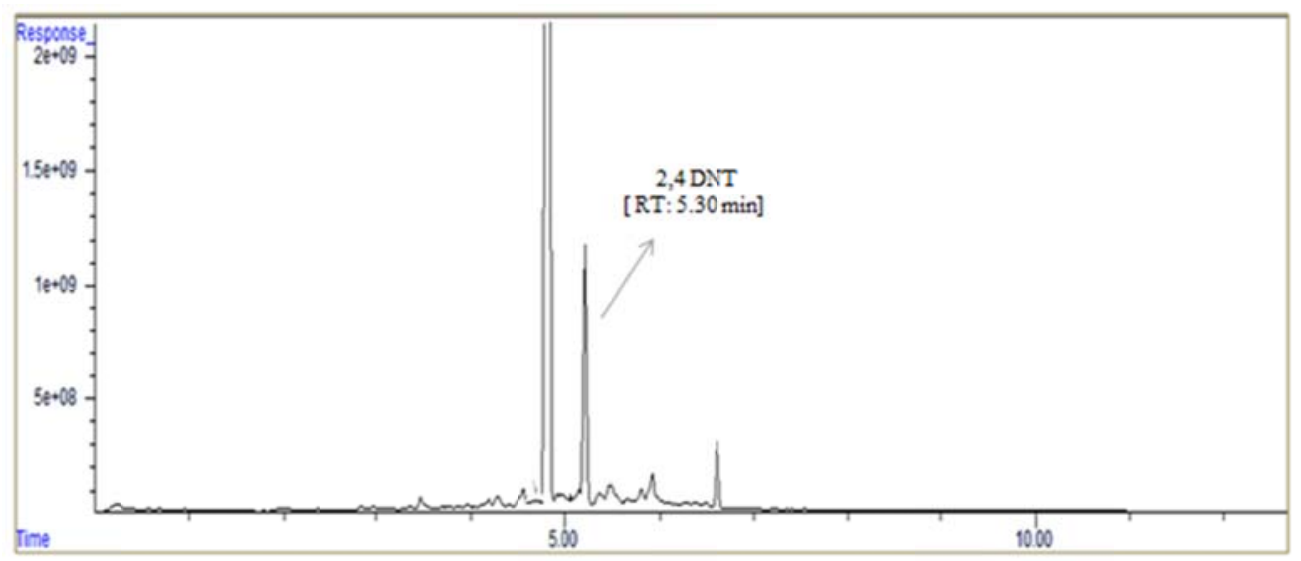

\section{Figure 36: Headspace SPME-GC/ECD of 2,4 DNT Collected at High Flow Rate at $30 \mathrm{sec}$}

\subsubsection{Sampling Time of 2,4 DNT Representative Volatile from TNT}

Trials of 2,4 -DNT target odorant from TNT at each flow rate for $60 \mathrm{sec}$ are depicted in Figure 37. After quantitative analysis was made, an average mass collected of the target odorant was of $6.02 \pm 0.2 \mathrm{ng}$ for low, $3.36 \pm 1.6 \mathrm{ng}$ for medium , and $7.01 \pm$ $1.9 \mathrm{ng}$ for high flow rate respectively (see Table 20). According to these results, the high flow rate at $60 \mathrm{sec}$ collected the greatest amount of the target odorant. However, reproducibility at this setting was not optimal. Statistical analysis was conducted (oneway Analysis of Variance, ANOVA; $\alpha=0.05)$. Upon completion of data collection it was found that when samples are collected for $60 \mathrm{sec}$ there is no significant difference in the amount of VOC collected among the three (3) different air flow collection rates. However, in order to establish an optimal parameter, a comparison of the $\%$ RSD between trials was obtained and it was found that at low flow rate the \% RD was less than 5\% showing to be the most appropriate parameter when compared with the others. 


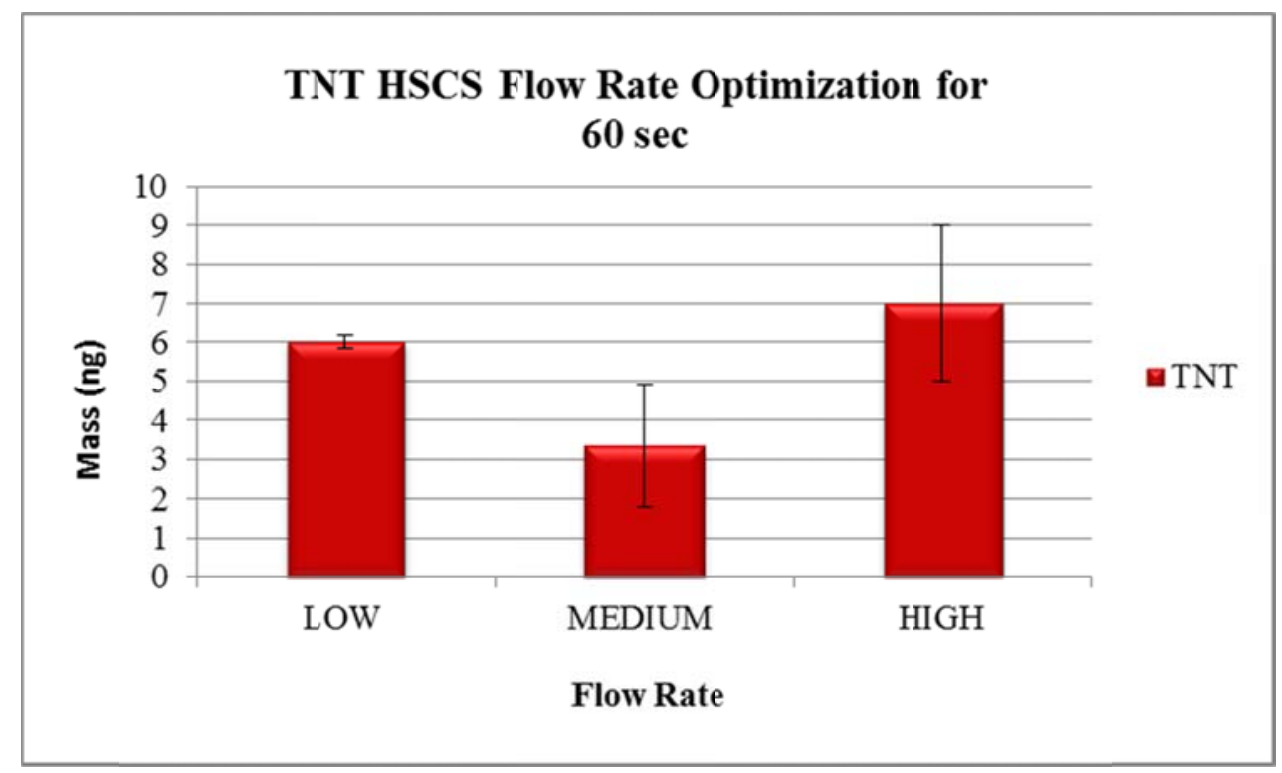

Figure 37: HSCS Collection of TNT Using Different Flow Rates for 60 seconds

Table 20: 2, 4-DNT Mass Average Collected form TNT at all Flow Rates for $60 \sec (n=3)$

\begin{tabular}{ccc}
\hline $\begin{array}{c}\text { Flow Rate } \\
\text { (60 sec) }\end{array}$ & $\begin{array}{c}\text { Average Mass } \\
\text { Collected (ng) }\end{array}$ & \multicolumn{2}{c}{ RSD (\%) } \\
\hline Low & $6.02 \pm 0.2$ & 5 \\
\hline Medium & $3.36 \pm 1.6$ & 80 \\
\hline High & $7.01+1.9$ & 49 \\
\hline
\end{tabular}

In order to establish the optimal collection time, a comparison of the already optimized flow rates at 30 and $60 \mathrm{sec}$ for TNT HSCS collection was performed (as seen in Figure 38). According to the experiment, the collection of TNT at low flow rate for 60 sec showed to have collected more of the target odorant and also provided better reproducibility. Therefore, the optimal parameter for HSCS collection of TNT explosive material is low flow rate for $60 \mathrm{sec}$ in which the average of scent mass collected was of $6.02 \pm 0.2 \mathrm{ng}$ with a RSD\% of $5 \%$ as seen in Table 21 . 
Statistical analysis was conducted (one-way Analysis of Variance, ANOVA; $\alpha=0.05$ ) and upon completion of data collection it was found that between 30 and $60 \mathrm{sec}$ there is no significant difference in the amount of VOC collected using low air flow collection rate.

Table 21: 2, 4-DNT Mass Average Collected from TNT at Low Flow Rate $(n=3)$

\begin{tabular}{ccc}
\hline $\begin{array}{c}\text { TIME } \\
\text { (sec) }\end{array}$ & $\begin{array}{c}\text { Average Mass } \\
\text { Collected (ng) }\end{array}$ & RSD (\%) \\
\hline $\mathbf{3 0}$ & $5.09+0.9$ & 33 \\
\hline 60 & $6.02+0.2$ & 5 \\
\hline
\end{tabular}

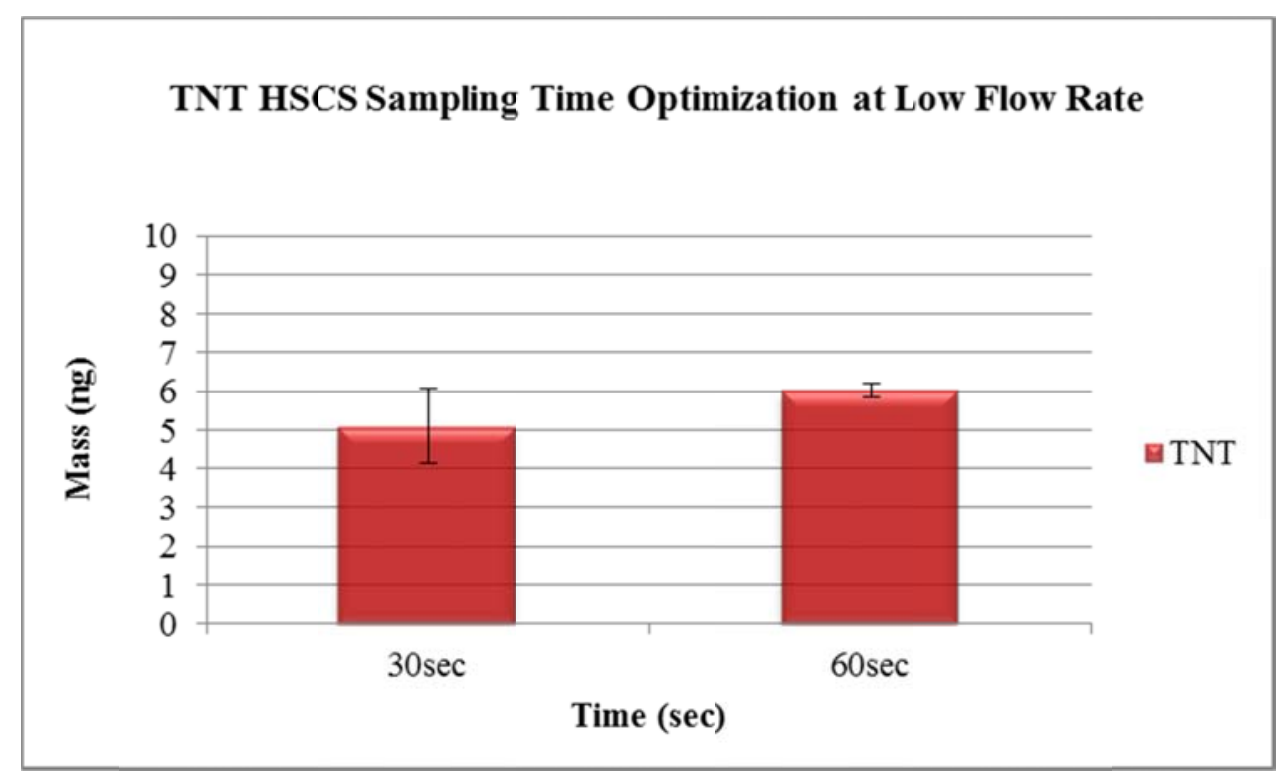

Figure 38: HSCS TNT Sampling Time Optimization at Low Flow Rate

As depicted in Figure 39, the chromatogram of one sample of TNT collected at the optimal established low flow rate and optimal sampling time of 60 seconds is shown in which 6.02 ng of 2,4 DNT mass scent was collected. 


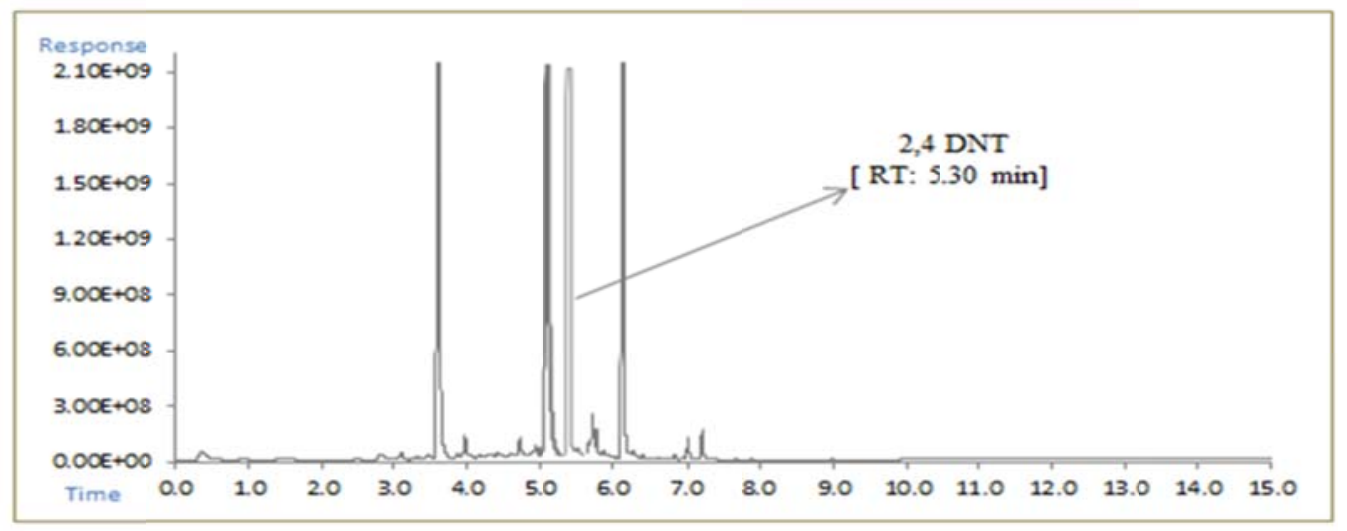

Figure 39: Headspace SPME-GC/ECD of TNT Collected at Low Flow Rate at 60 sec

\subsubsection{Sampling Time of Nitroglycerin Representative Volatile}

In order to establish the optimal collection time, HSCS collection of nitroglycerin (NG) was performed at the 60 second time setting (as seen in Figure 40 ). The same protocol for collection at $30 \mathrm{sec}$ was followed with a total of nine (9) trials. The samples were collected at indoor laboratory conditions $\left(22.4{ }^{\circ} \mathrm{C}\right.$ with a relative humidity of $56.7 \%)$.

According to the results, the mass of VOCs collected at high flow rate for $60 \mathrm{sec}$ was $0.34 \pm 0.05 \mathrm{ng}$ with a $\% \mathrm{RSD}$ of $48 \%$ as expressed in Table 22 . When compared with collection for $30 \mathrm{sec}$, it can be seen that the collection of NG at high flow rate for $30 \mathrm{sec}$ showed to have collected more of the target odorant as well as to have produced the best reproducibility value. The average mass of VOCs collected at this time was of $0.61 \pm$ $0.07 \mathrm{ng}$. Therefore, the optimal parameter for HSCS collection of double based smokeless powder is high flow rate for $30 \mathrm{sec}$ which produced the lowest \% RSD value of $33 \%$ as seen in Table 22 . 
Table 22: Nitroglycerin Mass Average Collected from Double Smokeless

Powder at High flow rate $(n=9)$

\begin{tabular}{ccc}
\hline $\begin{array}{c}\text { TIME } \\
\text { (sec) }\end{array}$ & $\begin{array}{c}\text { Average Mass } \\
\text { Collected (ng) }\end{array}$ & RSD (\%) \\
\hline $\mathbf{3 0}$ & $0.61 \pm 0.07$ & 33 \\
\hline $\mathbf{6 0}$ & $0.34+0.05$ & 48 \\
\hline
\end{tabular}

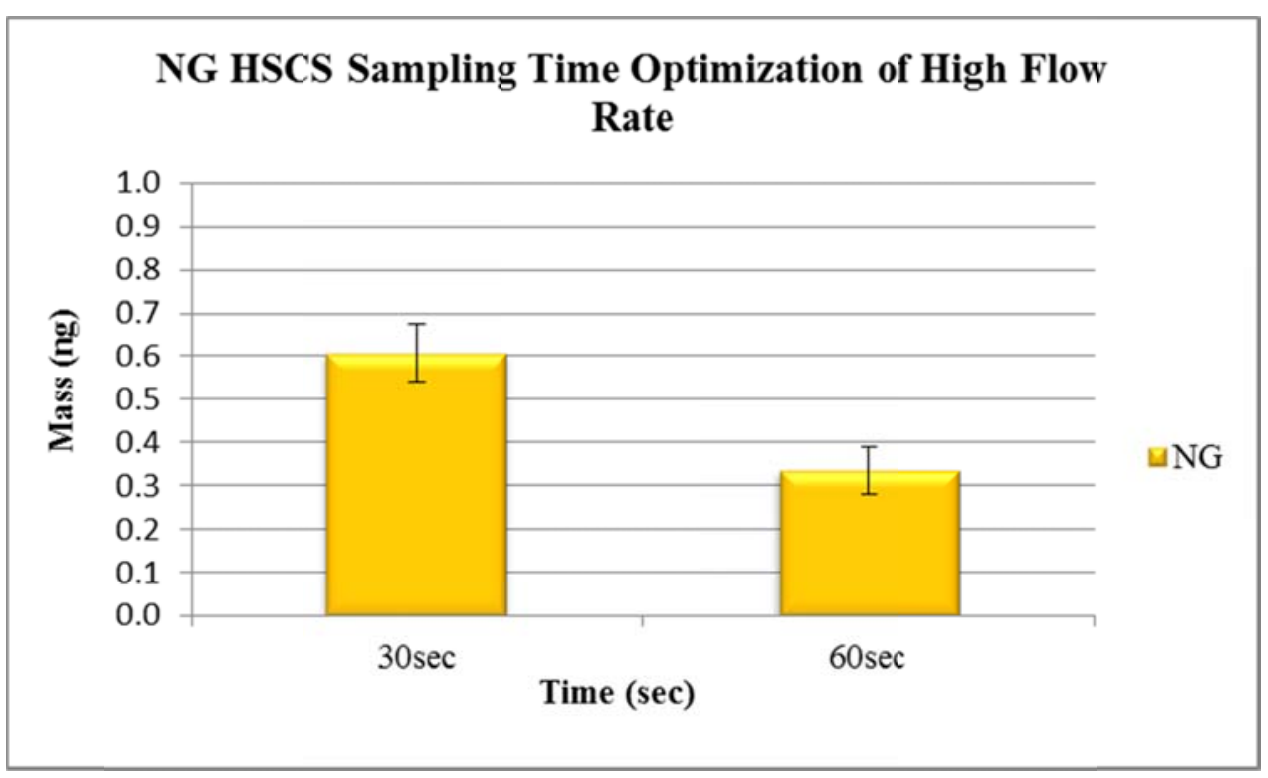

Figure 40: NG HSCS Sampling Time Optimization at High Flow Rate

As an example, Figure 41 depicts a chromatogram of one sample of double smokeless powder taken at the optimal parameters of high flow rate at $30 \mathrm{sec}$ in which $0.65 \mathrm{ng}$ of NG mass scent was collected. 


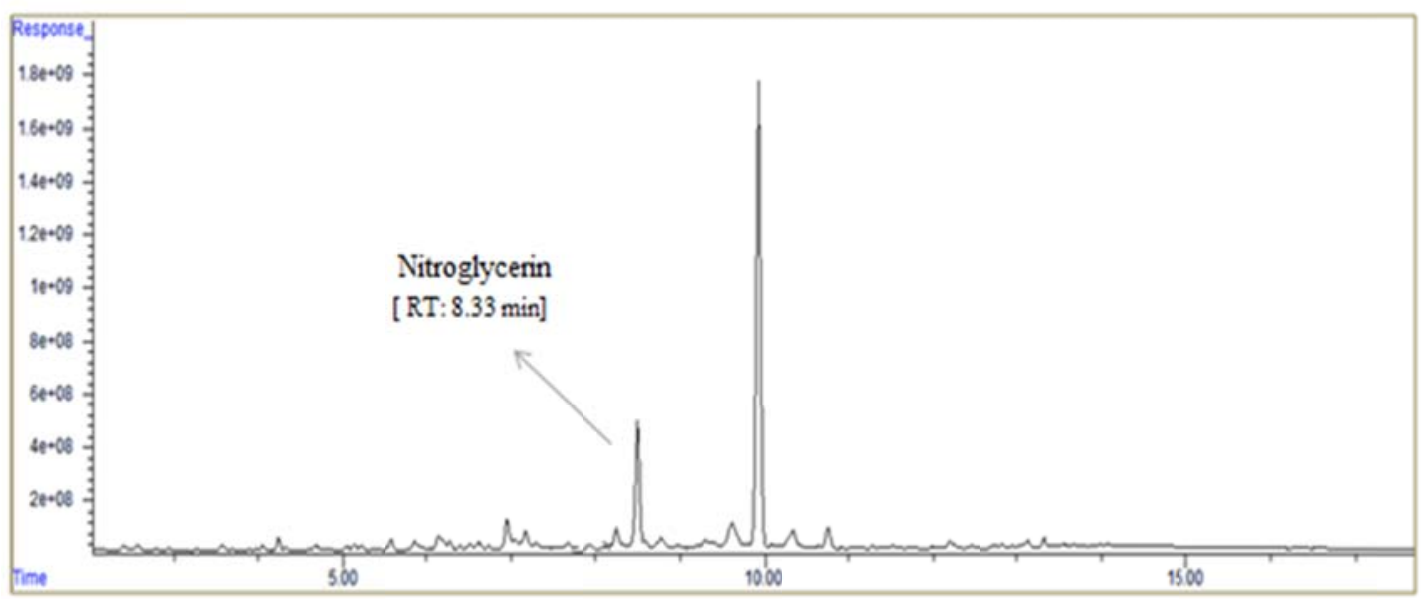

Figure 41: Headspace SPME-GC/ECD of NG Collected at High Flow Rate at 30 sec

\subsubsection{Comparison of HSCS Collection of 2,4-DNT Volatile from TNT and Single}

\section{Based Smokeless Powder}

In the present study, only one set of triplicates of single based smokeless powder were used for comparison purposes with TNT samples obtained from the ATF (source \#3).

The optimal parameters for HSCS collection of 2,4-dinitrotoluene (2,4-DNT) odorant from TNT explosive material was found to be low flow rate for $60 \mathrm{sec}$ in which the average of mass collected was $6.02 \pm 0.17 \mathrm{ng}$ with a RSD of 5\% . For the extraction of 2,4-DNT from single based smokeless powder, different optimal parameters of the HSCS were used. A high flow rate at $30 \mathrm{sec}$ that resulted in an average mass collected of $1.86 \pm 0.6$ ng with a RSD $\%$ of 60 (see Table 23 ). 
Table 23: Optimal Parameters for HSCS Collection of 2, 4-dinitrotoluene (n=3)

\begin{tabular}{ccccc}
\hline $\begin{array}{c}\text { Collection } \\
\text { Source }\end{array}$ & $\begin{array}{c}\text { Optimal Flow } \\
\text { Rate }\end{array}$ & $\begin{array}{c}\text { Optimal Time } \\
\text { (sec) }\end{array}$ & $\begin{array}{c}\text { Average Mass } \\
\text { Collected (ng) }\end{array}$ & RSD (\%) \\
\hline TNT & Low & 60 & $6.02 \pm 0.2$ & 5 \\
\hline $\begin{array}{c}\text { Single Based } \\
\text { Smokeless } \\
\text { Powder }\end{array}$ & High & 30 & $1.86 \pm 0.6$ & 60 \\
\hline
\end{tabular}

The high flow rate at $30 \mathrm{sec}$ was chosen as optimal parameters focused on the values of the $\%$ RSD which showed the least variation between samples. The results showed that optimal HSCS collection parameters of 2,4-dinitrotoluene depends on the composition of the explosive material. Different flow rates have been found for the collection of the target odorant from TNT and single based smokeless powder despite that almost equal amounts were utilized for collection (approx. 25g). This can be explained by factors such as the varying composition of the explosive material sources: one is a flake-like matrix while the other is a more homogeneous powder form (Figure 42). Another factor can be explained by the difference in the concentration of 2, 4-DNT within the two explosive materials. From the analytical laboratory perspective it can be said that HSCS collection heavily depends on the type of matrix being analyzed. Therefore recommendations for HSCS collection of 2, 4-DNT from TNT is low flow rate at 60 seconds and for single based smokeless powder is high flow rate at 30 seconds. 

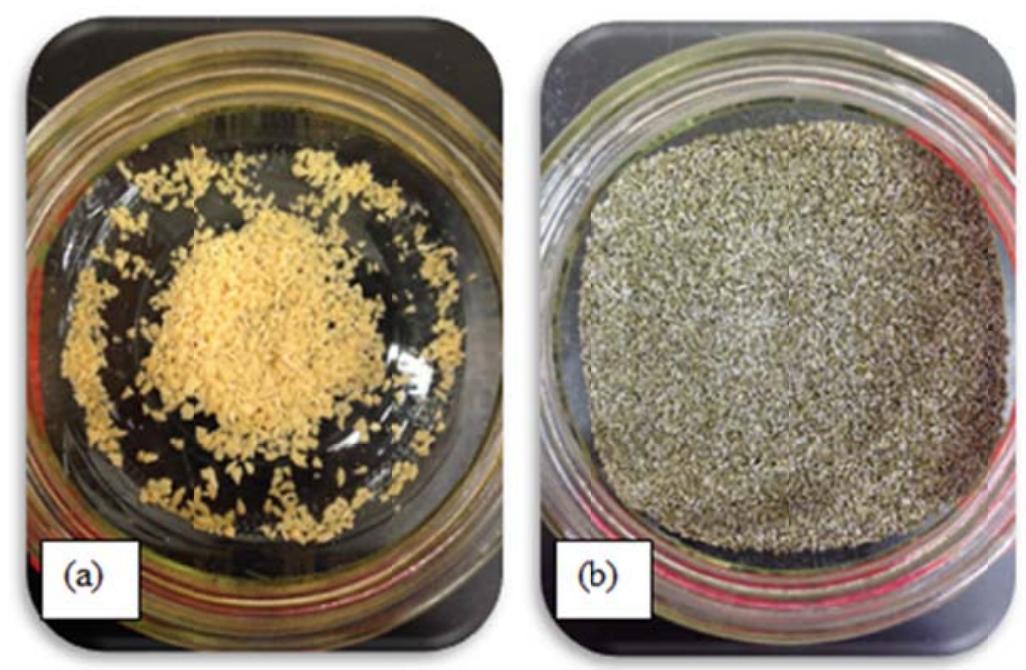

Figure 42: (a) TNT and (b) Single Based Smokeless Powder Matrix

\subsubsection{Conclusions}

HSCS dynamic collection plays a vital role in the effectiveness of trapping odorants. Dynamic collection can collect less of a target odorant most likely because the airflow volume passing through the HSCS opening at any given time can actually be causing the volatile to pass directly through the gauze medium reducing the overall amount collected on the gauze matrix. In addition, physical properties such as volatility affect the amount of odor obtained at a specific time setting and flow rate used during sample collection. The difference in mass of representative volatiles $2 \mathrm{E} 1 \mathrm{H}$ and DMNB collected at all locations can be explained by the difference in the concentration of both target odorants within the $\mathrm{C} 4$ explosive material provided by different sources. Composition $\mathrm{C} 4$ is the result of the combination of various ingredients which can be mixed in different ratios by manufacturers. As a result, the scent mass collected of $2 \mathrm{E} 1 \mathrm{H}$ 
and DMNB target volatiles will vary from source to source since specific concentrations of both are not given.

Other factors such as heat, humidity, and air flow can affect the rate of volatilization. Higher temperatures cause volatilization to proceed more quickly (since the kinetic energy of a molecule is proportional to its temperature) causing volatiles to be trapped faster into the absorbent media. In general, the higher the vapor pressure of a compound, the higher the tendency to vaporize and the lower the capacity to be retained by certain materials. In this study, three of the current representative odors under analysis: 2, 4dinitrotoluene $(2,4-\mathrm{DNT})$ from single based smokeless powder, nitroglycerin (NG) from double based smokeless powder, and DMNB from C4 explosive (Table 5) are odorants that exhibit low vapor pressures $\left(2.1 \times 10^{-4}, 2.4 \times 10^{-5}, 2.1 \times 10^{-3}\right.$ torr. at $25^{\circ} \mathrm{C}$ respectively) meaning that their capacity of volatilization is low and as a result cannot be efficiently trapped by the absorbent material during dynamic collection. In the case of $2 \mathrm{E} 1 \mathrm{H}$ which is highly volatile $\left(1.3 \times 10^{-1}\right.$ torr at $25^{\circ} \mathrm{C}$ respectively) it looks like volatilization is not an impairment for being effectively retained by the absorbent material. In addition, relative humidity can also be a factor since it depends on temperature. Higher temperature can increase the rate of volatilization and thus the amount of water vapor in the air. Therefore, low humidity also causes volatilization to be quicker because relative humidity will go down if the temperature is raised, and it will go up if the temperature is lowered meaning that molecules will absorb moisture as the relative humidity rises, and release moisture as it falls [105]. Another important factor refers to the presence of water vapor which may affect the adsorption of VOC molecules as they may compete with water molecules for the adsorption site [106]. In addition, 
Dukal gauze pads are made of cellulose backbone composed of hydroxyl groups with a weaving pattern. The molecular structure as well as the wave pattern of the gauze can affect the amount of volatile trapped/ released during collection [107]. The bonding or non-bonding of the volatiles to the backbones of the sorbent material can highly affect compound collection because compounds with high affinity to the collection material will bind too tightly, and those with no affinity will not bind to the sorbent material. The wave pattern of the gauze pad can yield significant loss of volatiles due to compound breakthrough through the sorbent medium.

\subsection{TASK 4: COMPARISON OF STATIC VS. DYNAMIC COLLECTION}

The aim of this task was to make a comparison of static versus dynamic mode of sample collection. Volatile organic compounds collected and evaluated using headspace SPME-GC/MS and/or SPME-GC/ECD at the HSCS optimal operational conditions (using both airflow optimal speed settings and time determined in Task 2 and Task 3) were compared with samples collected in the absence of the HSCS device. A static odor collection was performed on the same types of samples dynamically collected by conducting a time optimization test to determine the length of time $(30 \mathrm{~min}, 1 \mathrm{hr} ., 2 \mathrm{hrs}$, etc) a gauze pad efficiently trap the representative VOCs. In order to do this, a gauze pad was placed $2-3$ inches from the odor source for eventual SPME-GC/MS and/or SPMEGC/ECD analysis to establish which of the different time intervals of static mode

collection provided the greatest amount and optimal instrumental response of representative VOCs. 


\subsubsection{Static Collection of $2 \mathrm{E} 1 \mathrm{H}$ and DMNB Representative VOCs from C4}

In order to compare static mode versus dynamic mode of sample collection using the HSCS, the same samples of C4 explosive material utilized for dynamic collection were analyzed in the static mode by conducting a time optimization test. The protocol for the static collection of $2 \mathrm{E} 1 \mathrm{H}$ and DMNB representative odorants from $\mathrm{C} 4$ explosive material involved using the HSCS to only hold the gauze pad for collection of the target volatile organic compounds without applying any airflow at a distance of about 2 to 3 inches from the odor source (explosive material).

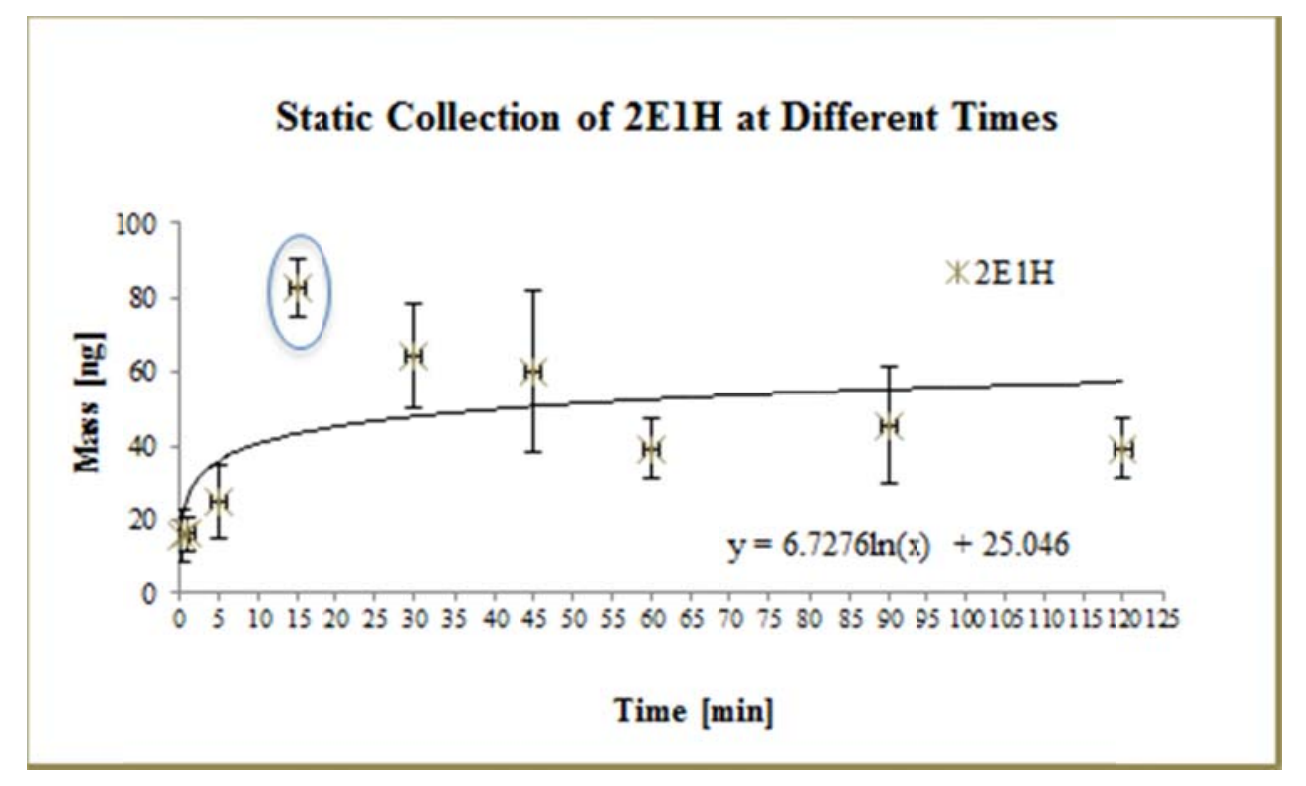

\section{Figure 43: Static Collection of 2E1H from C4 Explosive at Different Times}

Figure 43 shows the logarithmic relationship between mass and time for static mode collection of $2 \mathrm{E} 1 \mathrm{H}$ odorant. Initially, collection of $2 \mathrm{E} 1 \mathrm{H}$ started increasing gradually from 0.5 to 5 minutes until it reached $15 \mathrm{~min}$ where the greatest amount of the volatile was obtained. Eventually, collection is gradually reduced and at $60 \mathrm{~min}(1 \mathrm{hr}), 90$ $\min (1.5 \mathrm{hr})$, and $120 \mathrm{~min}(2 \mathrm{hr})$ there is no significant difference in the amount of 
odorant collected; indicating that after 60 min saturation is reached and regardless of how long the absorbent gauze material is exposed to the representative odorant only a certain amount of the odor is retained. The results show that equilibrium has been reached during the extraction process.

According to the results, the greatest collection of $2 \mathrm{E} 1 \mathrm{H}$ signature odorant was obtained when the gauze pad was exposed to the odor source for $15 \mathrm{~min}$ and subsequently volatile collection started to decrease over time. This means that during sampling procedures the volatile was effectively released from the $\mathrm{C} 4$ explosive material and transferred onto the gauze medium with minor headspace loss allowing a higher amount of $82.7 \pm 7.7 \mathrm{ng}$ to be collected with the least relative standard deviation \%RSD value of 16 (Table 24).

Table 24: Average Mass Collected of 2E1H by Static Mode at Different Times

\begin{tabular}{|ccc|}
\hline $\begin{array}{c}\text { Time } \\
(\mathbf{m i n})\end{array}$ & $\begin{array}{c}\text { Average Mass } \\
\text { Collected (ng) }\end{array}$ & \% RSD \\
\hline $\mathbf{0 . 5}$ & $15.9 \pm 6.9$ & 75 \\
\hline $\mathbf{1}$ & $16.1 \pm 4.6$ & 49 \\
$\mathbf{5}$ & $24.7 \pm 9.7$ & 68 \\
\hline $\mathbf{1 5}$ & $82.7 \pm 7.7$ & 16 \\
\hline $\mathbf{3 0}$ & $64.4 \pm 14$ & 37 \\
\hline $\mathbf{4 5}$ & $60.1 \pm 22$ & 62 \\
\hline $\mathbf{6 0}$ & $39.2 \pm 7.8$ & 34 \\
\hline $\mathbf{9 0}$ & $45.6 \pm 16$ & 60 \\
\hline $\mathbf{1 2 0}$ & $39.1 \pm 7.9$ & 35 \\
\hline
\end{tabular}


Static collection of DMNB showed that at 0.5 and 1 min collection of the odorant increased gradually, but at $5 \mathrm{~min}$ the amount of volatile collected was greater until it reached the $30 \mathrm{~min}$ collection interval. After $30 \mathrm{~min}$, collection was gradually reduced and at $60 \mathrm{~min}(1 \mathrm{hr}), 90 \mathrm{~min}(1.5 \mathrm{hr})$, and $120 \mathrm{~min}(2 \mathrm{hr})$ there is no significant difference in the amount of odorant collected as seen in Figure 44; meaning that after $60 \mathrm{~min}$ saturation is reached. The results show that equilibrium has been reached during the extraction process.

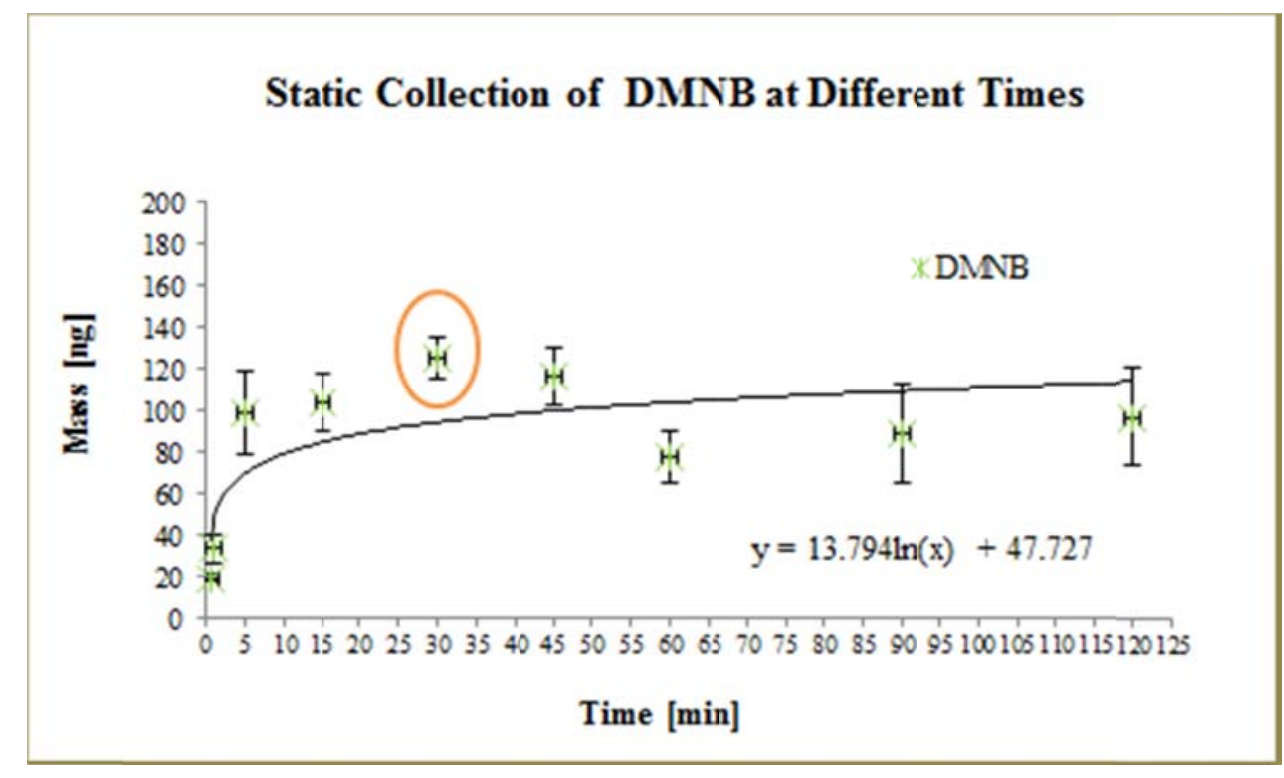

Figure 44: Static Collection of DMNB from C4 Explosive at Different Times

As seen in Table 25, optimal static collection time for DMNB from C4 explosive material is $30 \mathrm{~min}$ in which an average mass of $125 \pm 10 \mathrm{ng}$ with \% RSD of 14 was obtained. The longer static collection time required for DMNB when compared with $2 \mathrm{E} 1 \mathrm{H}$ can be explained by the fact that DMNB has lower volatility which requires a longer period of time for the vapor to be concentrated onto the sorbent material (Table 5). 
Table 25: Average Mass Collected of DMNB by Static Mode at Different Times

\begin{tabular}{|ccc|}
\hline $\begin{array}{c}\text { Time } \\
(\mathbf{m i n})\end{array}$ & $\begin{array}{c}\text { Average Mass } \\
\text { Collected (ng) }\end{array}$ & \% RSD \\
\hline $\mathbf{0 . 5}$ & $16.7 \pm 2.2$ & 20 \\
$\mathbf{1}$ & $33.7 \pm 6.6$ & 34 \\
$\mathbf{5}$ & $99.2 \pm 19$ & 34 \\
\hline $\mathbf{1 5}$ & $104 \pm 13$ & 23 \\
\hline $\mathbf{3 0}$ & $125 \pm 10$ & 14 \\
\hline $\mathbf{4 5}$ & $117 \pm 14$ & 20 \\
\hline $\mathbf{6 0}$ & $77.5 \pm 13$ & 28 \\
\hline $\mathbf{9 0}$ & $89.3 \pm 24$ & 46 \\
\hline $\mathbf{1 2 0}$ & $97.1 \pm 23$ & 41 \\
\hline
\end{tabular}

In relation to the actual amount of $2 \mathrm{E} 1 \mathrm{H}$ collected in static mode, analysis of variance (ANOVA) showed significant difference between the amounts collected at time intervals of $0.5,1$, and 5 min when compared with time intervals from 15 through 120 $\min (2 \mathrm{hr})$. But when intervals of 0.5 , and 5 min were compared to each other, analysis of variance showed no significant difference. The same result was obtained for comparison of time intervals between $15 \mathrm{~min}$ through $120 \mathrm{~min}(2 \mathrm{hr})$. On the other hand, analysis of variance (ANOVA) for DMNB collection in static mode, showed significant difference at 0.5 and 1 min time intervals when compared with the others. However; there was no significant difference when compared to each other. A similar result was obtained when time intervals from $5 \mathrm{~min}$ through $120 \mathrm{~min}(2 \mathrm{hr})$ were compared to each other.

According to the experiment, static collection of $\mathrm{C} 4$ explosive material as depicted in Figure 43 shows that for $2 \mathrm{E} 1 \mathrm{H}$ the greatest collection of signature odorant was obtained when the gauze pad was exposed to the odor source for $15 \mathrm{~min}$. Subsequently, 2E1H 
volatile collection started to decrease over time; meaning that during sampling procedures the volatile was effectively released from the $\mathrm{C} 4$ explosive material and transferred onto the gauze medium with minor headspace loss allowing a higher amount of volatile to be collected at 15 min time interval. On the other hand, greatest static collection of DMNB target odorant was obtained when the gauze pad was exposed to the odor source at $30 \mathrm{~min}$ and eventually started decreasing with increasing time ( as seen in Figure 44). The longer static collection time for DMNB when compared with 2E1H can be explained by the fact that DMNB has lower volatility and a slower dissipation rate in order to be successfully trapped through the gauze material as well as other environmental factors such as humidity and temperature.

The results obtained with these two representative odorants from $\mathrm{C} 4$ explosive material demonstrate that each target volatile behaves in a distinctive manner and that the interaction of the chemical with the collection medium (gauze matrix) as well as its intrinsic physical properties such as volatility affects the amount of odor obtained at specific time interval during sampling by the static mode.

\subsubsection{Comparison of Static vs. Dynamic Collection of 2E1H and DMNB Volatiles from $\mathrm{C} 4$}

As seen in Figure 45, dynamic collection of 2E1H outperforms static collection by providing higher amounts of the target volatile. It can be attributed to the fact that there is a better chance of trapping a greater amount of the odor within the gauze absorbent material by using a dynamic airflow mode. Table 26 shows that at high flow 
rate and at the sampling time of $30 \mathrm{sec}$ a greater amount of $2 \mathrm{E} 1 \mathrm{H}$ signature odorant was obtained.

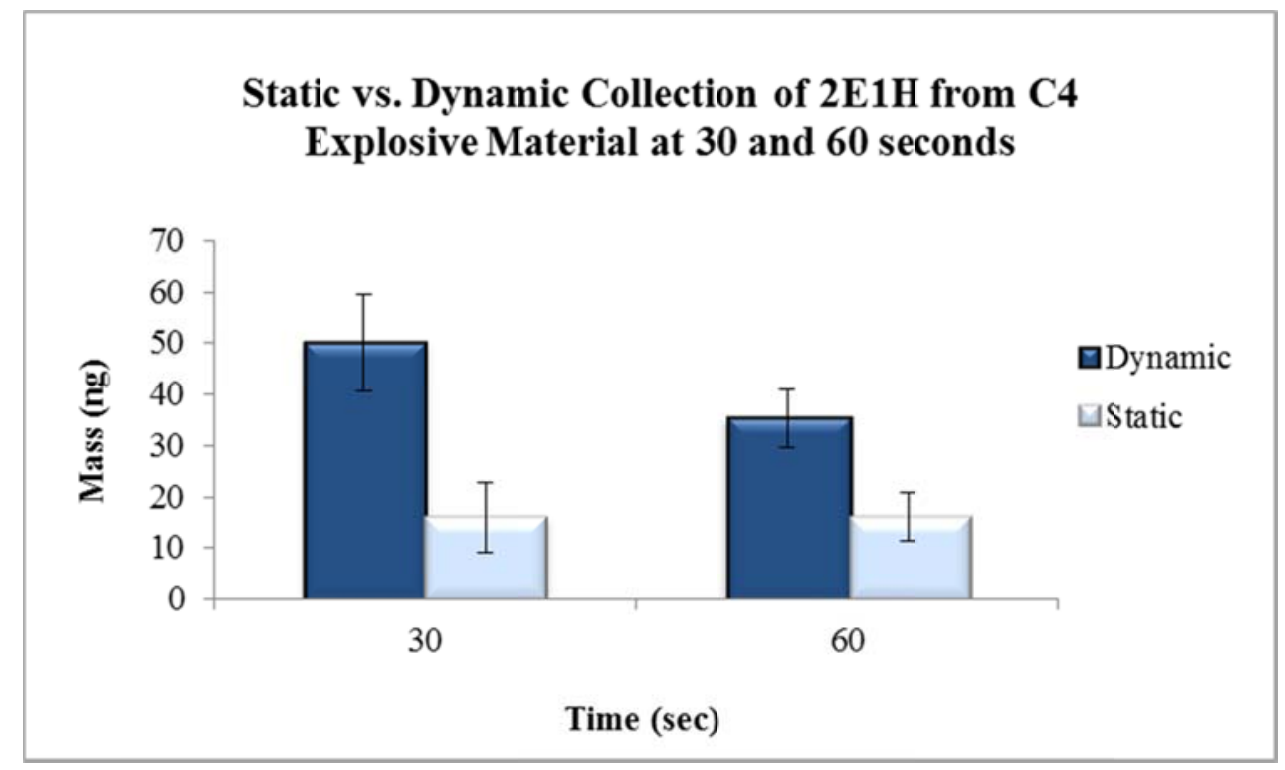

Figure 45: Static vs. Dynamic Collection of 2E1H odorant at 30 and 60 sec

Table 26: Static vs. Dynamic Collection of $2 \mathrm{E} 1 \mathrm{H}$ at 30 and $60 \mathrm{sec}(\mathrm{n}=3)$

\begin{tabular}{cccc}
\hline $\begin{array}{c}\text { Collection Time } \\
\text { (sec) }\end{array}$ & $\begin{array}{c}\text { Collection } \\
\text { Mode }\end{array}$ & $\begin{array}{c}\text { Average Mass } \\
\text { Collected (ng) }\end{array}$ & RSD (\%) \\
\hline $\mathbf{3 0}$ & Static & $15.9 \pm 6.9$ & 75 \\
& Dynamic & $50.2 \pm 9.5$ & 33 \\
\hline 60 & Static & $16.1 \pm 4.6$ & 49 \\
& Dynamic & $35.4 \pm 5.7$ & 28 \\
\hline
\end{tabular}

In contrast, (as seen in Figure 46) collection of DMNB target odorant was greater at static mode at $60 \mathrm{sec}$ sampling time in which $33.7 \pm 6.6 \mathrm{ng}$ were obtained with a $\%$ RSD value of 34 ( Table 27). This can be explained by the fact that DMNB has much lower volatility than $2 \mathrm{E} 1 \mathrm{H}$ (Table 5); meaning that there is less of the compound 
vaporized in the air to be trapped by the gauze material during static sampling. In addition, dynamic collection of DMNB collected less of the target odorant most likely because the airflow volume passing through the HSCS opening at any given time can actually be causing the volatile to pass directly through the gauze medium reducing the overall amount collected on the gauze. The lack of air flow movement during static mode collection does not allow the volatiles to pass through the gauze and as a result volatiles move from the explosive material onto the gauze medium with minor headspace loss.

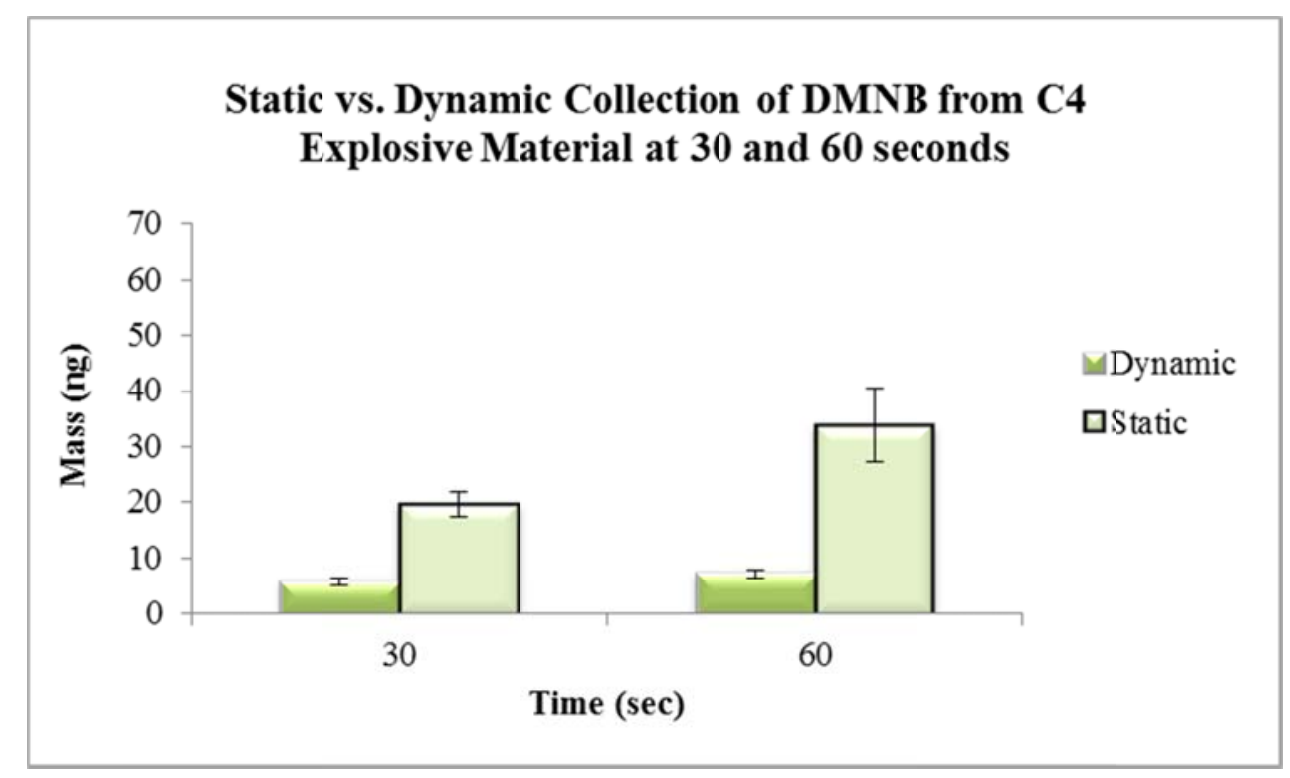

Figure 46: Static vs. Dynamic Collection of DMNB Odorant at 30 and 60 sec

Table 27: Static vs. Dynamic Collection of DMNB at 30 and $60 \mathrm{sec}$

\begin{tabular}{cccc}
\hline $\begin{array}{c}\text { Collection Time } \\
(\mathbf{s e c})\end{array}$ & $\begin{array}{c}\text { Collection } \\
\text { Mode }\end{array}$ & $\begin{array}{c}\text { Average Mass } \\
\text { Collected (ng) }\end{array}$ & RSD (\%) \\
\hline $\mathbf{3 0}$ & Static & $19.7 \pm 2.2$ & 20 \\
& Dynamic & $5.87 \pm 0.1$ & 4 \\
$\mathbf{6 0}$ & Static & $33.7 \pm 6.6$ & 34 \\
& Dynamic & $7.13 \pm 0.8$ & 20 \\
\hline
\end{tabular}


While it is possible to collect more odor using static collection, much greater extraction times are required than are available when performing dynamic collection. Static collection for $15 \mathrm{~min}$ and $30 \mathrm{~min}$ for $2 \mathrm{E} 1 \mathrm{H}$ and $\mathrm{DMNB}$ respectively provided greater amounts of each odor than the 30 seconds used for optimal collection in the dynamic mode. However, when comparing static and dynamic modes using the same sampling times (30 and $60 \mathrm{sec}$ ), dynamic mode does provide higher amounts of $2 \mathrm{E} 1 \mathrm{H}$ explosive odorant while static mode provided higher amounts of the DMNB target VOC as seen in Figure 45 and Figure 46.

\subsubsection{Static Collection of 2, 4-DNT Volatile from Single Based Smokeless Powder}

The 2, 4-DNT volatile present in single based smokeless powder was statically collected at different times in order to select the most suitable collection time to compare it with the target odor being dynamically collected. The protocol for static collection involved the use of the HSCS device to only hold the gauze pad at a distance of 2-3 inches from the odor source.

The results showed that at $0.5,1$, and 5 min static collection was minimal (as seen in Figure 47). But after 5 min, collection of the odorant started increasing gradually until 30 min time interval was reached. Eventually, collection of 2, 4 DNT odorant was mostly the same; meaning that after 30 min saturation is reached and no matter how long the absorbent gauze material is exposed to the representative odorant only certain amount of the odor is retained. The results show that equilibrium has been reached during the extraction process. 
According to the results, static collection of single based smokeless powder showed a higher mass scent collected at $120 \mathrm{~min}$ in which $5.23 \pm 0.9 \mathrm{ng}$ of the target odorant was obtained. However; based on the values of the relative standard deviation (\%RSD) the least variation between samples was obtained at 30 minutes when compared with the other time intervals as expressed in Table 28. Since equilibrium was reached at 30 minutes and \%RSD value was less; optimal static collection time for 2, 4-DNT from single based smokeless powder was determined to be $30 \mathrm{~min}$ in which an average mass of $5.05 \pm 0.5 \mathrm{ng}$ with $\% \mathrm{RSD}$ of 18 was obtained.

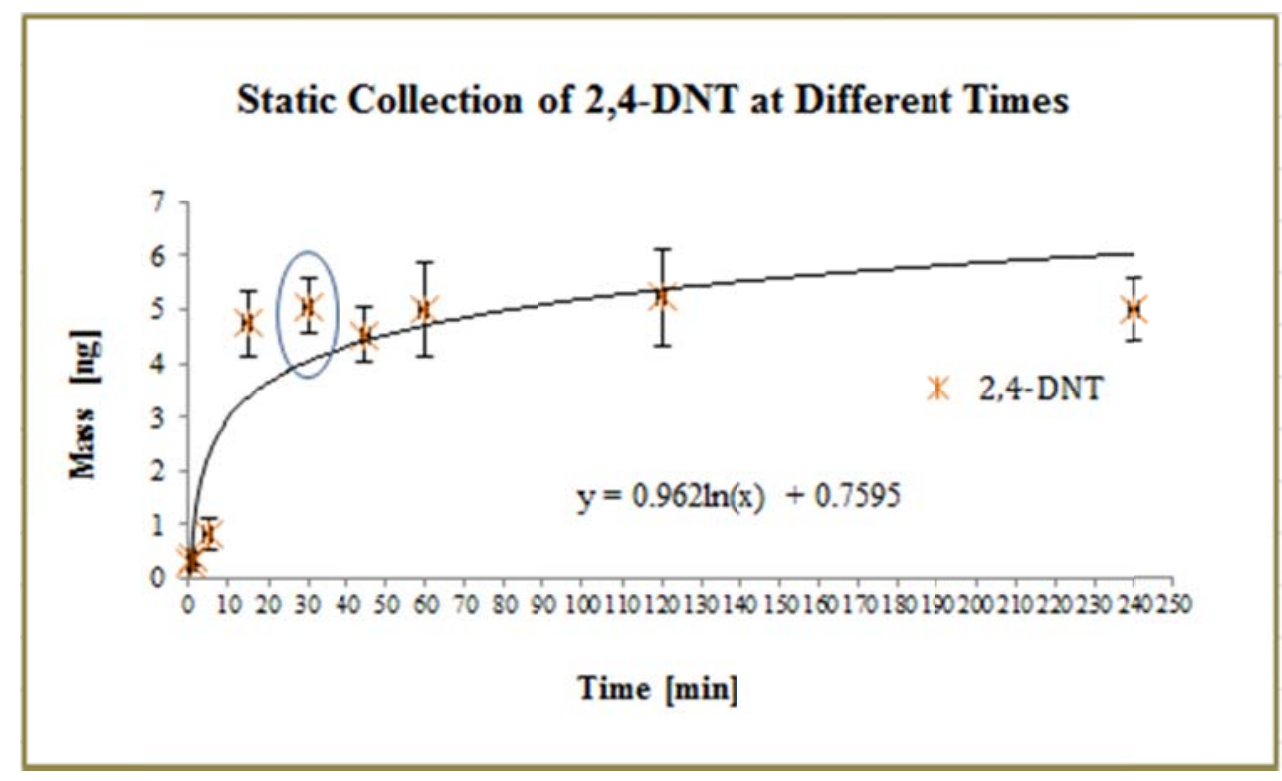

\section{Figure 47: Static Collection of 2,4-DNT from Single Based Smokeless Powder at Different Times}

In relation to the actual amount of 2, 4-DNT collected in static mode, analysis of variance (ANOVA) showed significant difference in the amounts collected at time intervals of $0.5,1$ and 5 min when compared with time intervals between 15 through 240 
minutes. But when intervals of $0.5,1$, and 5 min were compared to each other, analysis of variance showed no significant difference. The same result was obtained for comparison of time intervals between 15 through $240 \mathrm{~min}$.

Table 28: Average Mass Collected of 2, 4-DNT by Static Mode at Different Times

\begin{tabular}{|ccc|}
\hline $\begin{array}{c}\text { Time } \\
(\mathbf{m i n})\end{array}$ & $\begin{array}{c}\text { Average Mass } \\
\text { Collected (ng) }\end{array}$ & \% RSD \\
\hline $\mathbf{0 . 5}$ & $0.30 \pm 0.2$ & 83 \\
\hline $\mathbf{1}$ & $0.39 \pm 0.1$ & 52 \\
$\mathbf{5}$ & $0.84 \pm 0.3$ & 62 \\
\hline $\mathbf{1 5}$ & $4.74 \pm 0.6$ & 23 \\
\hline $\mathbf{3 0}$ & $5.05 \pm 0.5$ & 18 \\
\hline $\mathbf{4 5}$ & $4.52 \pm 0.5$ & 20 \\
\hline $\mathbf{6 0}$ & $5.00 \pm 0.9$ & 30 \\
\hline $\mathbf{1 2 0}$ & $5.23 \pm 0.9$ & 29 \\
\hline $\mathbf{2 4 0}$ & $5.00 \pm 0.6$ & 20 \\
\hline
\end{tabular}

Another experiment was performed in which a smaller amount of Hogdon 4896 smokeless powder $(10 \mathrm{~g})$ was utilized for static mode collection. Triplicate samples were prepared and sampled at laboratory conditions $\left(28^{\circ} \mathrm{C}\right.$ with a relative humidity of $\left.79.8 \%\right)$ along with a correspondent blank following the same protocol for static collection. The purpose of this experiment was to determine if a change in the amount of explosive material causes a change in the optimal static collection time.

As depicted in Table 29, 30 minutes is the optimal time for static collection mode of 2,4-DNT from single based smokeless powder. The same result as static analysis of $25 \mathrm{~g}$ was obtained (Table 28). The collection of 2,4 DNT odorant increases until it reaches equilibrium at 30 min to eventually decrease and later remind constant, meaning no 
matter how long the absorbent gauze material is exposed to the representative odorant only certain amount of the odor is retained.

Table 29: Average Mass Collected of 2,4-DNT (from 10g of single based smokeless powder) by Static Mode at Different Times $(n=3)$

\begin{tabular}{|ccc|}
\hline $\begin{array}{c}\text { Time } \\
(\mathbf{m i n})\end{array}$ & $\begin{array}{c}\text { Average Mass } \\
\text { Collected (ng) }\end{array}$ & \% RSD \\
\hline $\mathbf{5}$ & $0.41 \pm 0.2$ & 77 \\
\hline $\mathbf{1 5}$ & $0.98 \pm 0.2$ & 40 \\
\hline $\mathbf{3 0}$ & $2.41 \pm 0.1$ & 6 \\
\hline $\mathbf{4 5}$ & $1.89 \pm 0.1$ & 12 \\
\hline $\mathbf{6 0}$ & $2.00 \pm 0.2$ & 14 \\
\hline
\end{tabular}

\subsubsection{Comparison of Dynamic vs. Static Collection of 2,4-DNT Volatile from Single Based Smokeless Powder}

When static collection of 2,4-DNT representative odorant was obtained at 30 and 60 seconds, comparison with dynamic collection was performed. As depicted in Figure 48, triplicate samples of HSCS dynamic collection at high flow rate showed a higher mass scent collected for $30 \mathrm{sec}(0.5 \mathrm{~min})$ and $60 \mathrm{sec}(1 \mathrm{~min})$ when compared to the static collection at the same time interval.

The average mass collected of 2,4 DNT odorant from single based smokeless powder was $1.35 \pm 0.1 \mathrm{ng}$, and $5.05 \pm 0.5 \mathrm{ng}$ for HSCS high flow rate collection for $30 \mathrm{sec}$, and 30 min static mode determined to be the optimal collection times respectively. As seen in Figure 47, static mode shows greater collection of target VOC. This was as a result of the extended exposure time of $30 \mathrm{~min}$ used versus the time of $30 \mathrm{sec}$ used for collection in the dynamic mode. When comparing static and dynamic modes for the exact same 
extraction time, dynamic mode outperforms static collection by providing higher amounts of the target VOC as expressed in Figure 48and Table 30.

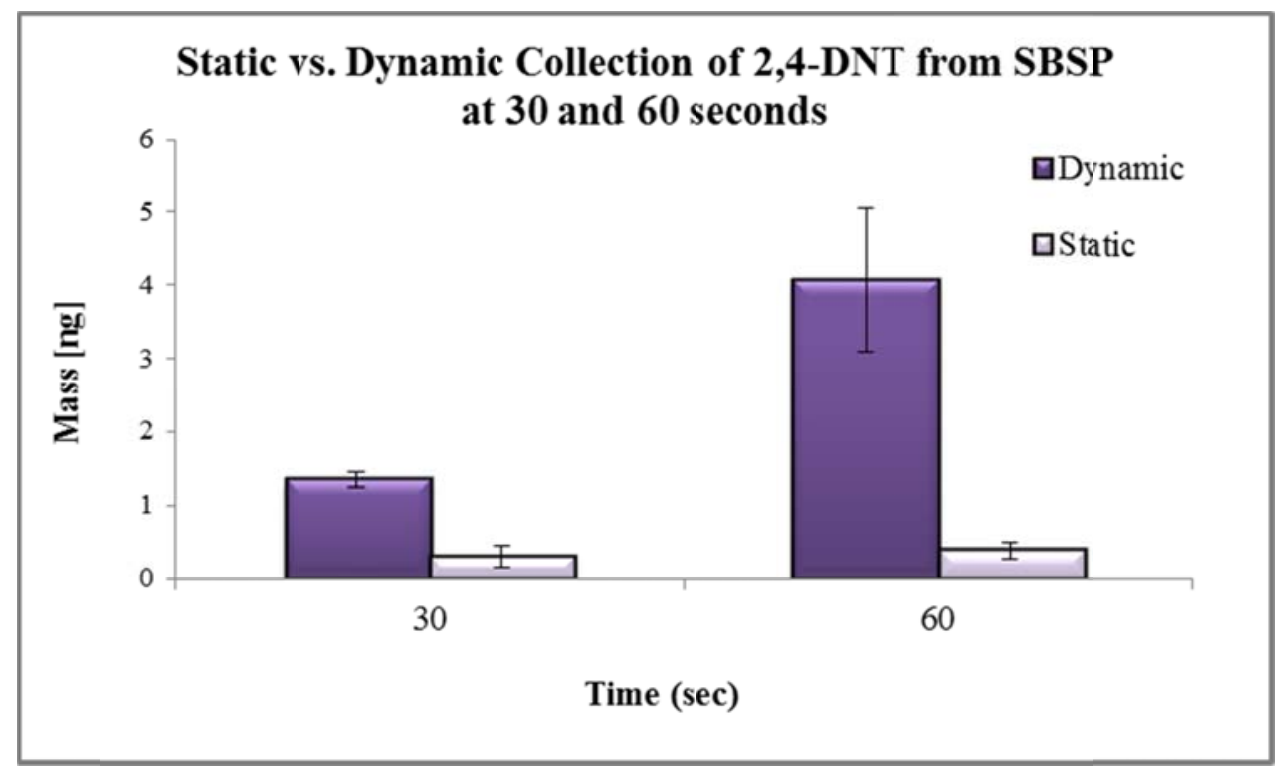

Figure 48: Static vs. Dynamic Collection of 2,4 DNT at 30 and 60 seconds

Table 30: Static vs. Dynamic Collection of 2,4 DNT at 30 and 60 seconds

\begin{tabular}{cccc}
\hline $\begin{array}{c}\text { Collection } \\
\text { Time }\end{array}$ & $\begin{array}{c}\text { Collection } \\
\text { Mode }\end{array}$ & $\begin{array}{c}\text { Average Mass } \\
\text { Collected (ng) }\end{array}$ & RSD (\%) \\
\hline $\mathbf{3 0}$ & Static & $0.30 \pm 0.2$ & 83 \\
& Dynamic & $1.35 \pm 0.1$ & 24 \\
$\mathbf{6 0}$ & Static & $0.39 \pm 0.1$ & 52 \\
& Dynamic & $4.07 \pm 0.9$ & 42 \\
\hline
\end{tabular}

\subsubsection{Static Collection of Nitroglycerin Volatile from Double Based Smokeless Powder}

In an effort to continue with the static collection of target volatiles from explosive material, the same triplicate samples of double based smokeless powder utilized for 
dynamic collection were analyzed by static mode. Nitroglycerin volatile from double based smokeless powder was statically collected at different times in order to select the most suitable collection time to compare it with the target odor being dynamically collected. The same protocol explained in section 5.6 was followed for the static collection of NG volatile.

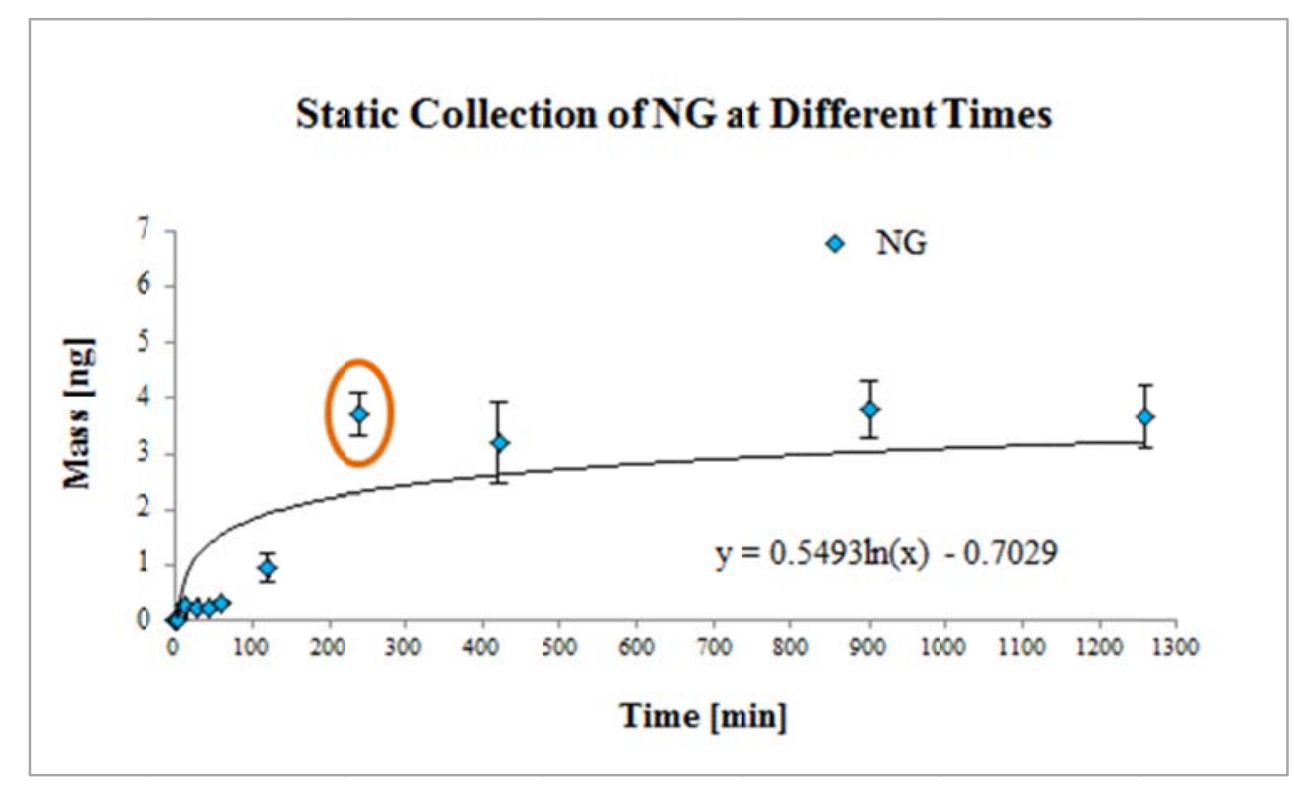

\section{Figure 49: Static Collection of NG Volatile from Double Based Smokeless Powder at Different Times}

As depicted in Figure 49, intervals of $0.5,1$, and 5 min yielded no instrumental detection of nitroglycerin meaning that the exposure time was not enough for the gauze pad to efficiently trap the target volatile. After 15 min interval, collection of the volatile started increasing gradually and at $240 \mathrm{~min}(4 \mathrm{hr})$ the greatest amount of $3.71 \pm 0.4 \mathrm{ng}$ was collected with the least RSD\% value of 18 as seen in Table 31. For the remaining time intervals, the amount of volatile collected did not change meaning that saturation of 
the sorbent material has been reached. Therefore, the optimal static collection time for nitroglycerin from double based smokeless powder was determined to be $240 \mathrm{~min}(4 \mathrm{hr})$.

In relation to the actual amount of nitroglycerin collected in static mode, analysis of variance (ANOVA) showed significant difference between the amounts collected at time intervals of $15,30,45,60$, and 120 min when compared with time intervals at 240 (4 hr) through $1260 \mathrm{~min}(21 \mathrm{hr})$. But when intervals of 15, 30, 45, 60 min were compared to each other, analysis of variance showed no significant difference. The same result was obtained for comparison of time intervals between 240 (4 hr) through $1260 \mathrm{~min}(21 \mathrm{hr})$.

Table 31: Average Mass Collected of Nitroglycerin by Static Mode at Different Times

\begin{tabular}{|ccc|}
\hline $\begin{array}{c}\text { Time } \\
(\mathbf{m i n})\end{array}$ & $\begin{array}{c}\text { Average Mass } \\
\text { Collected (ng) }\end{array}$ & \% RSD \\
\hline $\mathbf{1 5}$ & $0.24 \pm 0.1$ & 47 \\
\hline $\mathbf{3 0}$ & $0.24 \pm 0.1$ & 21 \\
\hline $\mathbf{4 5}$ & $0.23 \pm 0.1$ & 38 \\
\hline $\mathbf{6 0}$ & $0.32 \pm 0.1$ & 27 \\
\hline $\mathbf{1 2 0}$ & $0.95 \pm 0.3$ & 48 \\
\hline $\mathbf{2 4 0}$ & $3.71 \pm 0.4$ & 18 \\
\hline $\mathbf{4 2 0}$ & $3.20 \pm 0.7$ & 39 \\
\hline $\mathbf{9 0 0}$ & $3.81 \pm 0.5$ & 24 \\
\hline $\mathbf{1 2 6 0}$ & $3.67 \pm 0.5$ & 26 \\
\hline
\end{tabular}

\subsubsection{Comparison of Dynamic vs. Static Collection of Nitroglycerin Volatile from Double Based Smokeless Powder}

When static collection of nitroglycerin representative volatile was obtained at 30 and $60 \mathrm{sec}$, comparison with dynamic collection was performed. As depicted in Figure 
50, HSCS dynamic collection at high flow rate showed the amount of volatile organic compounds collected for $30 \mathrm{sec}(0.5 \mathrm{~min})$ and $60 \mathrm{sec}(1 \mathrm{~min})$ when compared to the static collection at the same time intervals in which the signature odorant was not detected (ND).

As seen in Table 32, $0.65 \pm 0.1 \mathrm{ng}$ of nitroglycerin volatile from double based smokeless powder were collected by dynamic mode for $30 \mathrm{sec}$ with the least RSD\% value of 27 (triplicate samples).

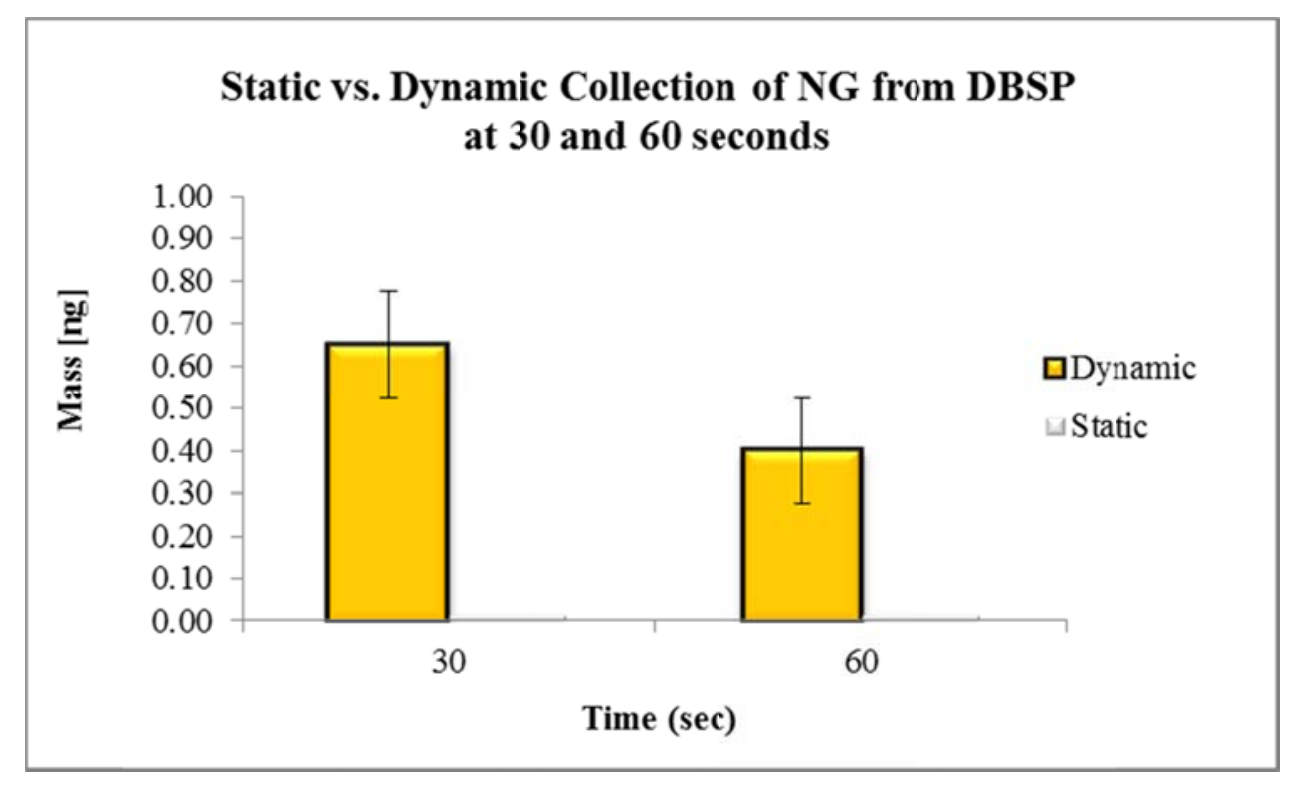

Figure 50: Static vs. Dynamic Collection of Nitroglycerin at 30 and 60 sec

The average mass collected for triplicate samples was $0.65 \pm 0.1 \mathrm{ng}$, and $3.71 \pm$ $0.4 \mathrm{ng}$ for HSCS high flow rate collection for $30 \mathrm{sec}$, and $240 \mathrm{~min}(4 \mathrm{hr})$ determined to be the optimal collection times respectively. Figure 49 shows that a greater amount of the explosive odorant was obtained when samples were collected in a static mode for 240 $\min (4 \mathrm{hr})$. However, this was as a result of the extended exposure time of $240 \mathrm{~min}(4 \mathrm{hr})$ used versus the time of $30 \mathrm{sec}$ used for optimal collection in the dynamic mode. When 
comparing static and dynamic modes for the exact same extraction time (30 and $60 \mathrm{sec}$ ), dynamic mode provided higher amounts of the target VOC while static mode did not collect any of the explosive volatile (see Table 32 and Figure 50).

Table 32: Static vs. Dynamic Collection of Nitroglycerin at 30 and 60 seconds

\begin{tabular}{|c|c|c|c|}
\hline $\begin{array}{c}\text { Collection } \\
\text { Time }\end{array}$ & $\begin{array}{c}\text { Collection } \\
\text { Mode }\end{array}$ & $\begin{array}{l}\text { Average Mass } \\
\text { Collected (ng) }\end{array}$ & RSD (\%) \\
\hline \multirow[t]{2}{*}{30} & Static & ND & N/A \\
\hline & Dynamic & $0.65 \pm 0.1$ & 27 \\
\hline \multirow[t]{2}{*}{60} & Static & ND & N/A \\
\hline & Dynamic & $0.40 \pm 0.1$ & 45 \\
\hline
\end{tabular}

\subsubsection{Conclusions}

Dynamic and static collection modes were the two different methods used in this study to collect samples. It has been demonstrated that these modes efficiently collected target volatiles from real explosive material in different amounts. In general, when comparing both modes, static collection showed to produce a greater amount of target VOCs which was as a result of the extended exposure time. However, nitroglycerin from double based smokeless powder was not detected at 30 and $60 \mathrm{sec}$ of static collection. It implies that 30 and 60 seconds was not enough time for the volatile to be trapped by the absorbent material and a longer time of exposure was required. The vapor pressure of NG $\left(2.1 \times 10^{-5}\right.$ Torr at $\left.25^{\circ} \mathrm{C}\right)$ is low and therefore was the cause for NG not to be efficiently trapped by the sorbent material (Table 5). In addition, static analysis was made in triplicates, therefore only a set of triplicates samples from each explosive material dynamically collected was used for comparison purposes. 


\subsection{EVALUATION OF LONGEVITY OF REPRESENTATIVE VOCS AND SELECTION OF AN OPTIMAL STORAGE CONTAINMENT SYSTEM}

This task evaluated the persistence of explosive odors on a sorbent material following collection using optimized operational conditions for the HSCS for each type of chemical volatile evaluated. The persistence of explosive odors was evaluated to determine how long the VOCs are detectable instrumentally. As the objective of this research was to produce feasible training aids for operant canine use, it is of key importance to measure the length of time these novel training aids have detectable odor in the headspace, which can then be related to the ability of the canine to efficiently detect this target odorant when in training. In this task, samples were stored at room temperature and aged for time periods ranging from hours to over one month after sampling (e.g. $1 \mathrm{hr}$, $4 \mathrm{hr}, 1$ day, 1 week, 4 weeks). After the aging period, headspace evaluation of these samples was conducted using SPME-GC/MS and/or SPME-GC/ECD. Longevity of the training aid was determined based on time when explosive odors were no longer detectable by the instrument. The selection of a storage containment system suitable for all the collected samples using the optimized operational conditions for the HSCS for each type of chemical odorant was also a critical factor evaluated. The collection material is a 4" x 4" cotton gauze pad using the optimal operational conditions (flow rate and time) of the HSCS for the collection of chemical odorants will be stored in different containers. The storage containment systems tested include plastic and aluminized bags (heat sealed and ziploc) as well as glass jars. After sample collection, headspace evaluation of these samples was analyzed using SPME-GC/MS and/or SPME-GC/ECD and optimal storage containment system was determined based on the least amount of 
background as well as maximum odor containment in any given sample after instrumental analysis.

\subsubsection{Persistence of Target Volatiles 2E1H and DMNB and Optimal Storage Containment System}

The optimal storage containment system was studied by conducting a comparison

of the mass average among a set of triplicate samples collected at high flow rate for 60 seconds. The plotted results of mass loss vs. time in hours yielded the permeation rate of each odor through the three different containment systems.

After quantitative analysis, the results obtained for the longevity study of $\mathrm{C} 4$ explosive material samples showed that samples collected in aluminum bags allowed the complete dissipation of both odorants 2E1H and DMNB during the first hour. The result highlights the low capacity of the aluminum bag to hold (trap) the target volatiles within its membrane thus indicating a high porosity of this particular matrix (see Figure 51 and Figure 52). On the other hand, $40 \mathrm{ml}$ vials were able to retain $2 \mathrm{E} 1 \mathrm{H}$ and DMNB volatiles at a greater amount up to the third week of the study. In the case of ziploc bags, the persistence of $2 \mathrm{E} 1 \mathrm{H}$ disappeared by the first week, while DMNB was completely gone after the 24 hour storage period as depicted in Figure 51. 


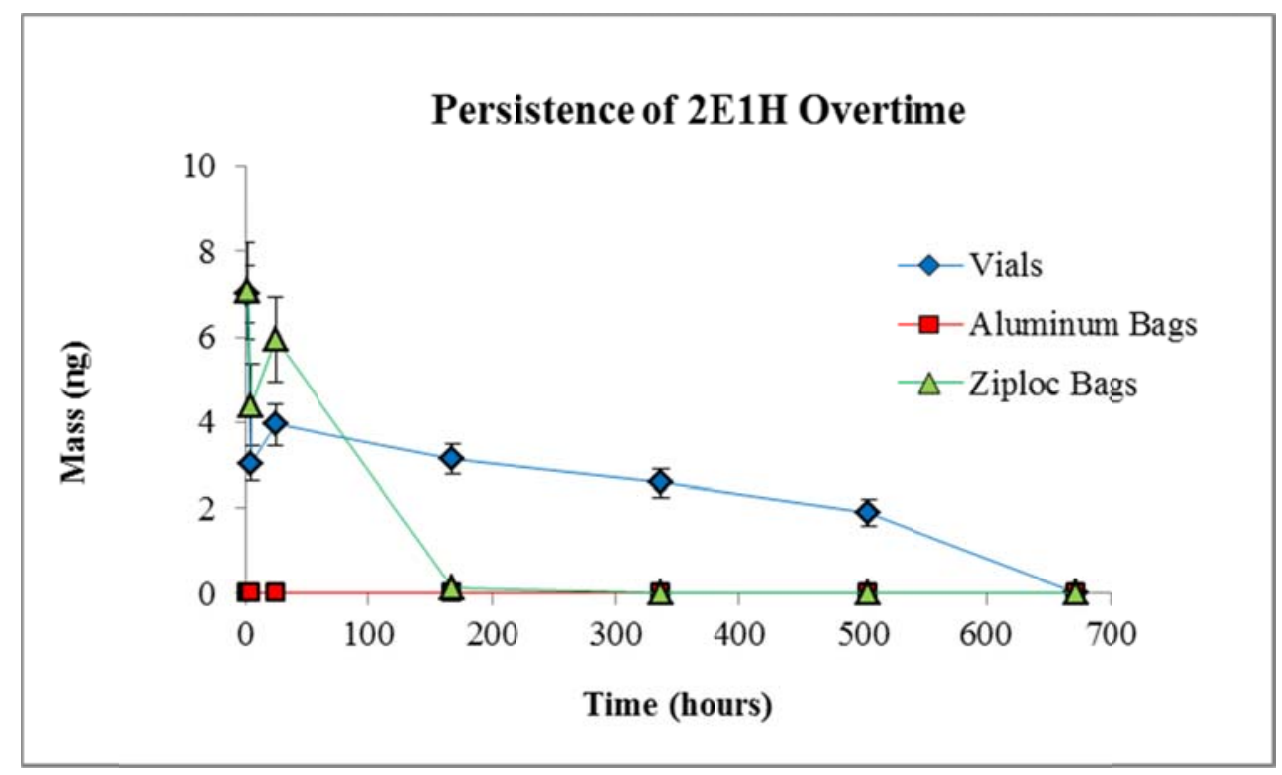

Figure 51: Persistence of 2E1H Target Odorant Overtime in the Tested Containers

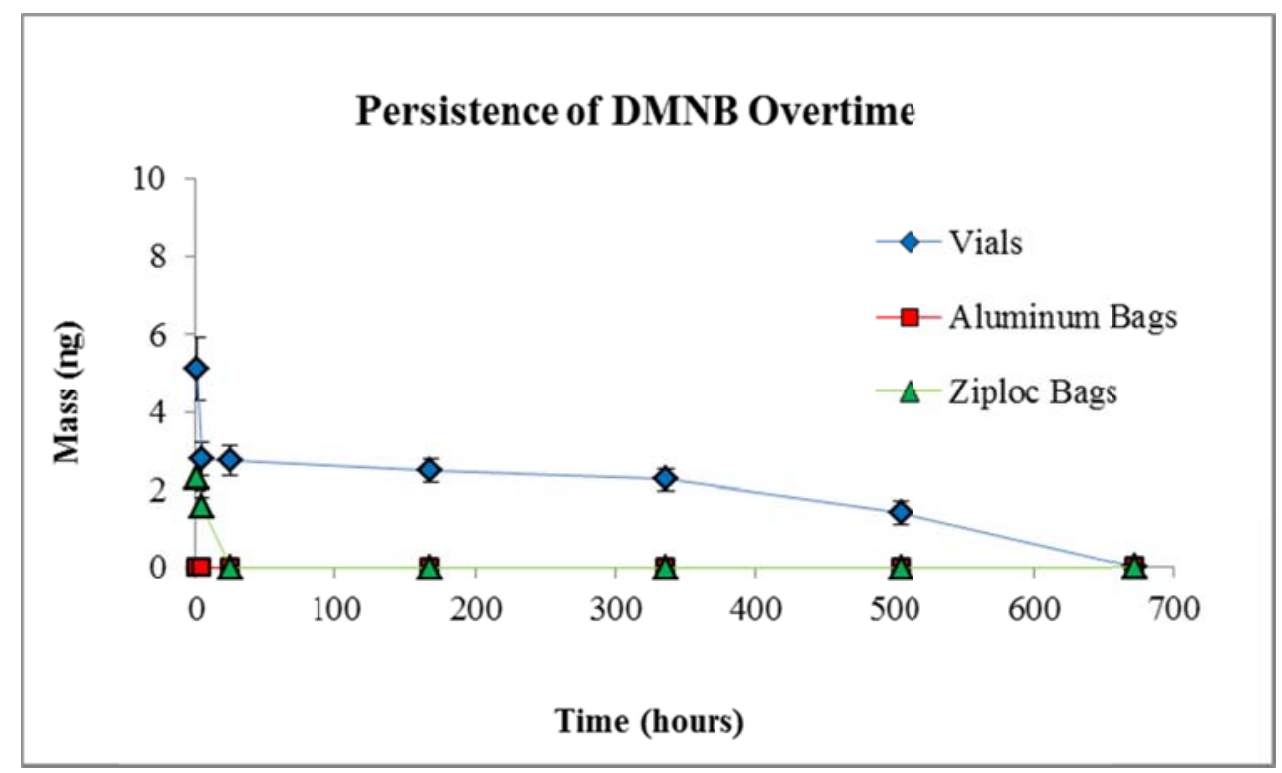

Figure 52: Persistence of DMNB Target Odorant Overtime in the Tested Containers

The results depicted in Table 33 and Table 34 show that 2E1H was collected at a greater amount than DMNB in vials and dissipated faster for the selected period of analysis. It appears that DMNB is slowly released from the gauze pad material before permeating in 
the environment, as seen in Figure 52. According to the results, the initial average mass collected in vials was $7.01 \pm 0.7 \mathrm{ng}$ for $2 \mathrm{E} 1 \mathrm{H}$ and $5.10 \pm 0.8 \mathrm{ng}$ for DMNB respectively. The persistence of both volatiles in glass vials by the end of the one month evaluation period decreased significantly (as seen in Figure 51 and Figure 52) but overall retained more of the target VOCs for a longer period of time than either the aluminum or ziploc bags.

Table 33: Average Mass Collected of 2E1H from C4 Explosive over a Month

\begin{tabular}{cccc}
\hline \multicolumn{4}{c}{ AVERAGE MASS COLLECTED (ng) } \\
\hline $\begin{array}{c}\text { TIME } \\
\text { (hours) }\end{array}$ & Vials & Aluminum bags & Ziploc bags \\
$\mathbf{1}$ & $7.01 \pm 0.7$ & ND & $7.08 \pm 1.1$ \\
$\mathbf{4}$ & $3.03 \pm 0.4$ & ND & $4.40 \pm 0.9$ \\
$\mathbf{2 4}$ & $3.94 \pm 0.5$ & ND & $5.93 \pm 1.0$ \\
$\mathbf{1 6 8}$ & $3.12 \pm 0.3$ & ND & ND \\
$\mathbf{3 3 6}$ & $2.57 \pm 0.3$ & ND & ND \\
$\mathbf{5 0 4}$ & $1.89 \pm 0.3$ & ND & ND \\
$\mathbf{6 7 2}$ & ND & ND & ND \\
\hline
\end{tabular}

Table 34: Average Mass Collected of DMNB from C4 Explosive over a Month

\begin{tabular}{|cccc}
\hline \multicolumn{4}{c}{ AVERAGE MASS COLLECTED (ng) } \\
\hline $\begin{array}{r}\text { TIME } \\
\text { (hours) }\end{array}$ & Vials & Aluminum bags & Ziploc bags \\
$\mathbf{1}$ & $5.10 \pm 0.8$ & ND & $2.32 \pm 0.4$ \\
$\mathbf{4}$ & $2.77 \pm 0.4$ & ND & $1.60 \pm 0.2$ \\
$\mathbf{2 4}$ & $2.74 \pm 0.4$ & ND & ND \\
$\mathbf{1 6 8}$ & $2.48 \pm 0.3$ & ND & ND \\
$\mathbf{3 3 6}$ & $2.25 \pm 0.3$ & ND & ND \\
$\mathbf{5 0 4}$ & $1.41 \pm 0.3$ & ND & ND \\
$\mathbf{6 7 2}$ & ND & ND & ND \\
\hline
\end{tabular}

The persistence of the target odorants $2 \mathrm{E} 1 \mathrm{H}$ and DMNB in aluminum bags was null while in ziploc bags (double zipper) persistence of both volatiles was seen only up to a week. According to the results, the initial average mass collected in ziploc bags was 7.08 
$\pm 1.1 \mathrm{ng}$ for $2 \mathrm{E} 1 \mathrm{H}$ and $2.32 \pm 0.4 \mathrm{ng}$ for DMNB (Table 33and Table 34). The persistence of $2 \mathrm{E} 1 \mathrm{H}$ odorant in ziploc bags remained for one week in which $5.93 \pm 1.0 \mathrm{ng}$ were obtained. Volatile DMNB in ziploc bags only persisted for $24 \mathrm{hr}$ with an average mass collected of $1.60 \pm 0.2 \mathrm{ng}$. After these time intervals, both volatiles were no longer detected instrumentally. As explained above, glass vials are the most appropriate storage container for preservation of $2 \mathrm{E} 1 \mathrm{H}$ and DMNB VOCs from $\mathrm{C} 4$ explosive material, as it shows to retain both compounds for a longer period of time while providing a storage medium with highly reduced contamination when compared with aluminum and ziploc bags containment systems (Figure 53).

Figure 53 shows representative chromatograms produced from one (1) $\mathrm{hr}$ storage of gauze pads in glass vials, aluminum bags (heat sealed), and ziploc bags (double zipper). The presence of the volatiles $2 \mathrm{E} 1 \mathrm{H}$ and DMNB from $\mathrm{C} 4$ explosive material in glass vials and ziploc bags are compared with the $10 \mathrm{ng}$ standard (shown by the red arrow). In this Figure, the effects of storage in glass vials as well as aluminum and ziploc bags were the production of long-chain cycloalkanes which were present as a result of the chemical composition of the gauze pad (shown by the blue arrow).

For samples stored in aluminized bags, 2E1H and DMNB odorants were not detected (ND) as expressed in Figure 51 and Figure 52. This can be attributed to the fact that these matrices were all sealed utilizing a heat sealer which may have caused an enhanced presence of contaminating compounds originating from alumina composition. Some of the compound classes detected during chromatographic analysis included longchain cycloalkanes, alkanes, aldehydes, acid-methyl esters, and alcohols that increased in amount for analysis of samples stored longer than a $4 \mathrm{hr}$ time interval. The process of 
heat sealing may have contributed to the creation and/or release of many of these compounds which could have competed with the target compounds when being extracted with the SPME fiber preventing the proper detection of the expected odorants or causing these target odorants to be below detection threshold.

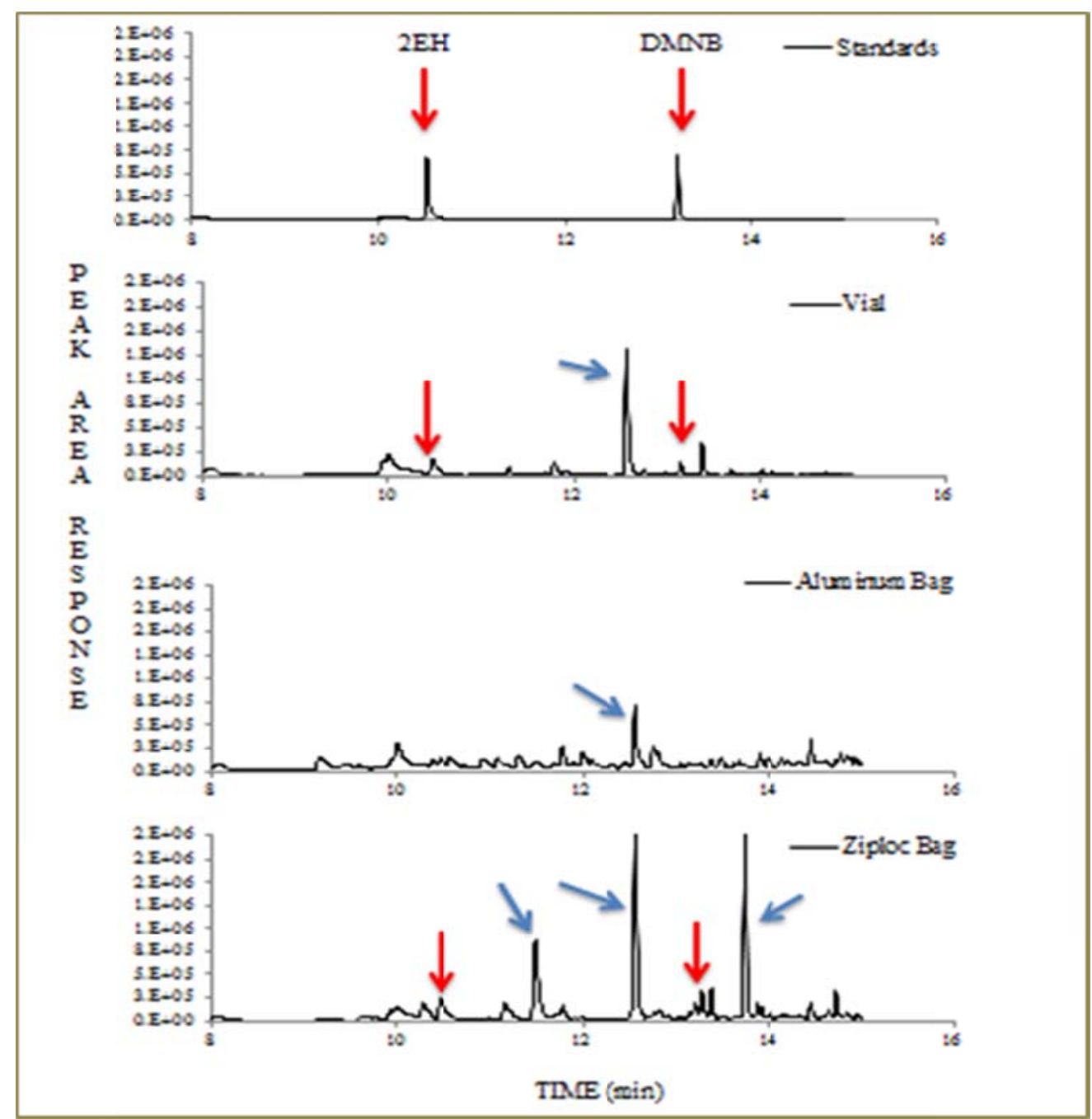

Figure 53: Comparison of Different Storage Containers after One (1) hr Storage 


\subsubsection{Persistence of Target Volatiles 2,4-DNT and NG and Optimal Storage Containment System}

The study of optimal storage containers involved chromatographic analysis of aged samples from one (1) hr, four (4) hr, and $24 \mathrm{hr}$ to over a month. However; in order to identify if there was a significant change in the persistence of the odorants in a $24 \mathrm{hr}$ period; a comparison of odor was performed between one (1), four (4) and $24 \mathrm{hr}$ intervals. A set of triplicate samples with a corresponding blank were dynamically collected at atmospheric conditions $\left(21.8^{\circ} \mathrm{C}\right.$ with a relative humidity of $\left.53.6 \%\right)$ from single and double based smokeless powders for each of the time intervals selected for comparison and placed in $40 \mathrm{ml}$ glass vials. As depicted in Figure 54 and Figure 55, persistence of $\mathrm{NG}$ and 2,4-DNT target odorants during the three (3) time intervals remains similar. Since SPME analysis of both target odorants requires $21 \mathrm{hr}$ extraction time; then analysis of aged samples for all the three (3) tested containers was performed from one (1) hr to one (1) week time interval. Statistical analysis (one-way Analysis of Variance, ANOVA; $\alpha=0.05$ ) showed no significant differences in the amounts of target odorants collected from single and double based smokeless powders for the three (3) time intervals. 


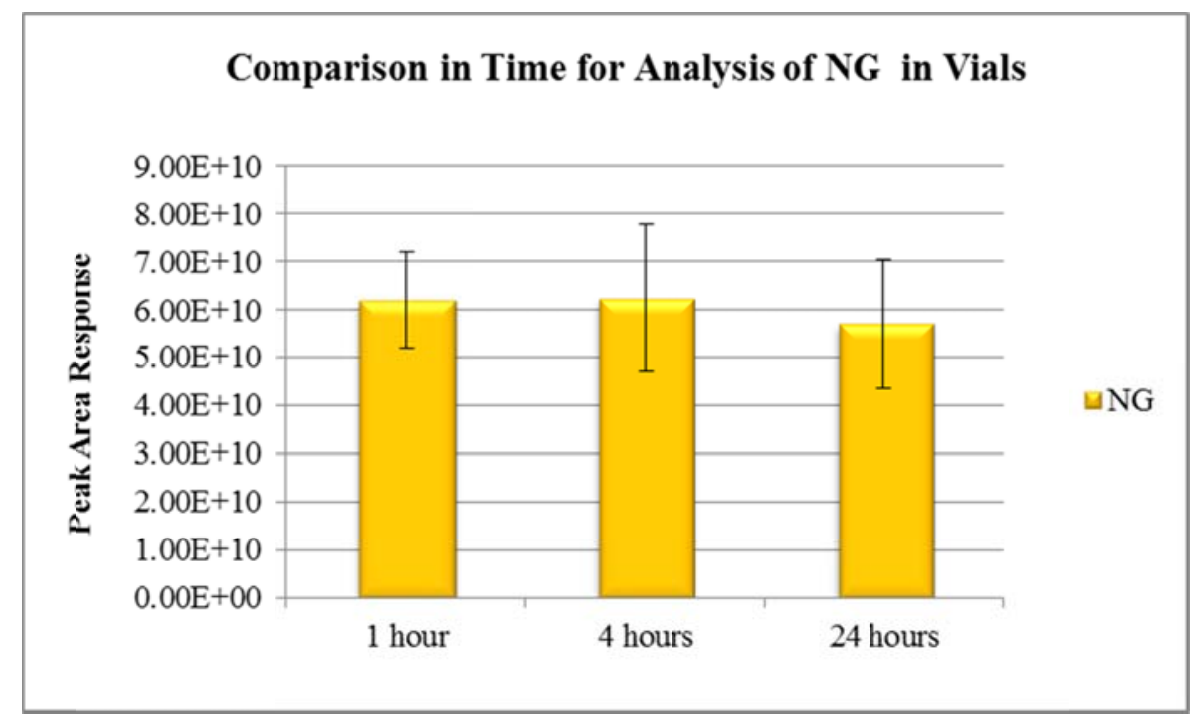

Figure 54: Comparison of NG Samples Aged in Vials at one (1) hr, four (4) hr, and $24 \mathrm{hr}$

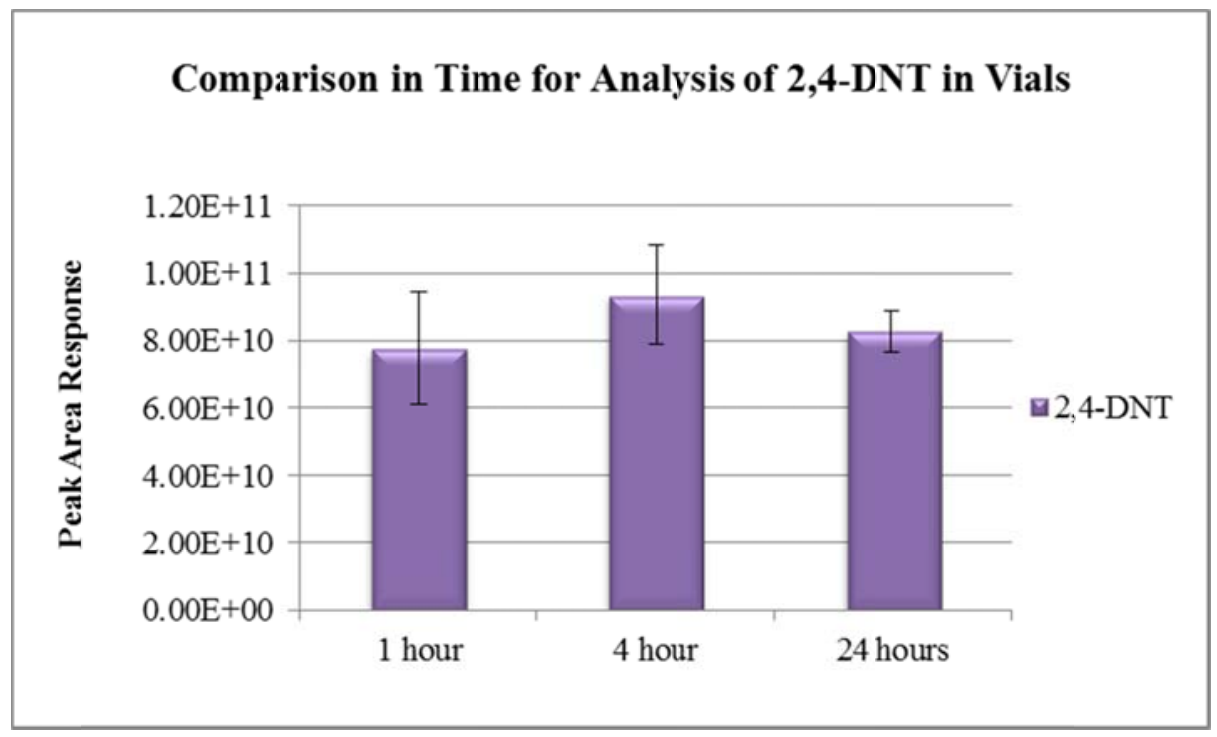

Figure 55: Comparison of 2,4-DNT Samples Aged in Vials at one (1) hr, four (4) hr, and $24 \mathrm{hr}$

Analysis was based on the peak area which is proportional to the amount of the compound that was present. The optimal storage containment was studied by conducting a comparison of the average of the peak areas found in a set of triplicate samples collected at high flow rate for $30 \mathrm{sec}$ for each of the different storage containment 
systems under study. The plotted results of mass loss vs. time in days allowed for a calculation of permeation rate of each odor through the three different containment systems.

After quantitative analysis, the results obtained for the longevity study of double based smokeless powder samples showed that during the first week of analysis the odorant was only partially released into the headspace of each storage container. This is believed to be as a result of the odorant adsorbing strongly on the cotton gauze and only being partially released over time. However, as more time was allowed, the signature odorant was released at a greater amount which later on decreased due to dissipation (Figure 56). From all the containers tested, $40 \mathrm{ml}$ vials has shown to be able to retain NG odorant at a greater amount followed by aluminum heat sealed bags and lastly ziploc bags.

The initial average mass collected was $2.07 \pm 0.2 \mathrm{ng}$ in glass vials, $0.51 \pm 0.1 \mathrm{ng}$ in aluminum bags (heat sealed), and $0.28 \pm 0.1 \mathrm{ng}$ for ziploc bags (double zipper) respectively. The persistence of the target odorant by the end of the month was $1.02 \pm 0.2$ ng in glass vials, $0.38 \pm 0.2 \mathrm{ng}$ in aluminum bags (heat sealed), and $0.21 \pm 0.1 \mathrm{ng}$ for ziploc bags (double zipper) respectively as depicted in Table 35 ). From these results it can be concluded that the dissipation rate of the target odorant is very slow for the selected period of analysis. It appears that the target odorant is slowly released from the gauze pad material before getting lost in the environment. As seen in Figure 56 it can be concluded that glass vials are the most appropriate storage container for the NG signature odorant as that container results in minimal loss of the odorant overtime. 


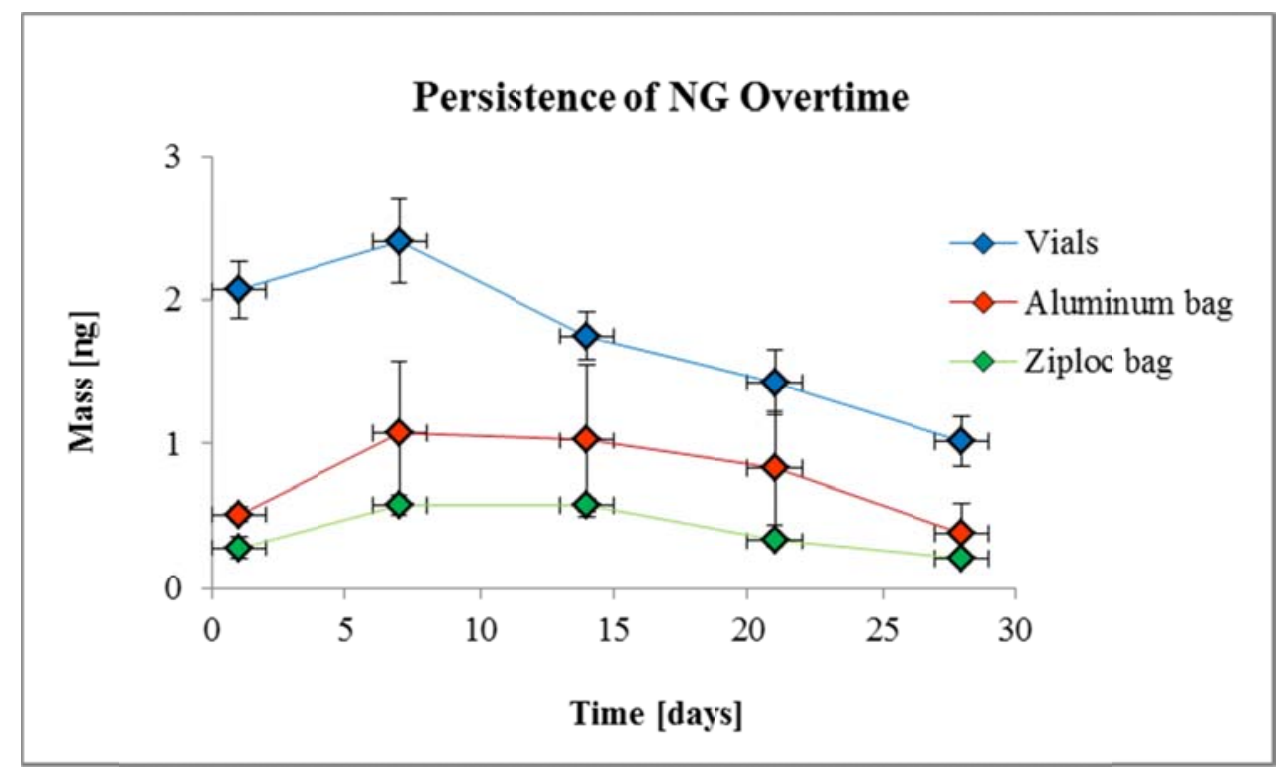

Figure 56: Persistence of NG Target Odorant Overtime in the Tested Containers

Table 35: Average Mass Collected of NG from Double Based Smokeless Powder over a Month

\begin{tabular}{cccc}
\hline \multicolumn{4}{c}{ AVERAGE MASS COLLECTED (ng) } \\
\hline $\begin{array}{c}\text { TIME } \\
\text { (days) }\end{array}$ & Vials & Aluminum bags & Ziploc bags \\
$\mathbf{1}$ & $2.07 \pm 0.2$ & $0.51 \pm 0.1$ & $0.28 \pm 0.8$ \\
$\mathbf{7}$ & $2.41 \pm 0.3$ & $1.08 \pm 0.5$ & $0.58 \pm 0.1$ \\
$\mathbf{1 4}$ & $1.75 \pm 0.2$ & $1.02 \pm 0.5$ & $0.56 \pm 0.1$ \\
$\mathbf{2 1}$ & $1.43 \pm 0.2$ & $0.83 \pm 0.4$ & $0.33 \pm 0.1$ \\
$\mathbf{2 8}$ & $1.02 \pm 0.2$ & $0.38 \pm 0.2$ & $0.21 \pm 0.1$ \\
\hline
\end{tabular}

The results for the longevity study of 2,4-DNT odorant from single based smokeless powder showed that for glass vials and aluminum bags, retention of the odorant increases for the first week, then remains the same for the following weeks and eventually starts decreasing during third week (21 days). On the other hand, persistence 
of 2, 4-DNT odorant in ziploc bags decreases for the first week and eventually remains constant until week three (3) when it starts decreasing.

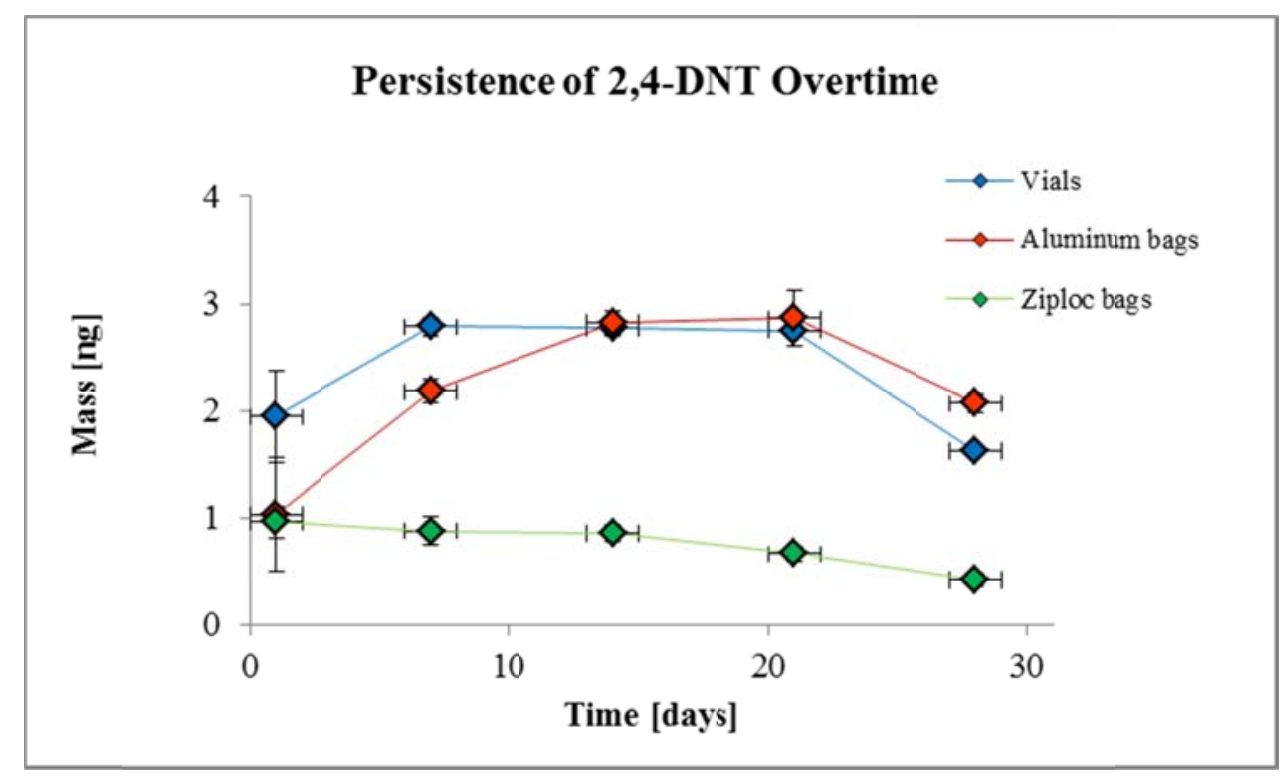

Figure 57: Persistence of 2, 4-DNT Target Odorant Overtime in the Tested Containers

Table 36: Average Mass Collected of 2,4-DNT from Single Based Smokeless Powder over a Month

\begin{tabular}{cccc}
\hline \multicolumn{4}{c}{ AVERAGE MASS COLLECTED (ng) } \\
\hline $\begin{array}{c}\text { TIME } \\
\text { (days) }\end{array}$ & Vials & Aluminum bags & Ziploc bags \\
$\mathbf{1}$ & $1.94 \pm 0.6$ & $1.03 \pm 0.5$ & $0.96 \pm 0.1$ \\
$\mathbf{7}$ & $2.78 \pm 0.1$ & $2.18 \pm 0.1$ & $0.88 \pm 0.1$ \\
$\mathbf{1 4}$ & $2.77 \pm 0.1$ & $2.83 \pm 0.1$ & $0.86 \pm 0.1$ \\
$\mathbf{2 1}$ & $2.75 \pm 0.1$ & $2.86 \pm 0.3$ & $0.66 \pm 0.1$ \\
$\mathbf{2 8}$ & $1.62 \pm 0.1$ & $2.07 \pm 0.1$ & $0.42 \pm 0.1$ \\
\hline
\end{tabular}

According to the results, as well as for persistence of NG, 2, 4-DNT was collected at a greater amount in glass vials followed by aluminum bags (heat sealed) and ziploc bags (double zipper). The initial average mass collected was $1.94 \pm 0.6 \mathrm{ng}$ for glass vials, 
$1.03 \pm 0.5 \mathrm{ng}$ for aluminum bags (heat sealed), and $0.96 \pm 0.1 \mathrm{ng}$ for ziploc bags (double zipper) respectively.

The persistence of the target odorant 2,4 DNT by the end of the month was $1.62 \pm$ $0.1 \mathrm{ng}$ for glass vials, $2.07 \pm 0.1 \mathrm{ng}$ for aluminum bags (heat sealed), and $0.42 \pm 0.1 \mathrm{ng}$ for ziploc bags (double zipper) respectively (as depicted in Table 36). The results indicate that the dissipation rate of the target odorant is extremely slow for vials and aluminum bags for the selected period of analysis. It appears that the target odorant is slowly released from the gauze pad material from glass vials for the first three (3) weeks before dissipating into the environment. Analysis of aluminum bags showed that the persistence of the target odorant increases overtime. This can be attributed to interfering compounds related to the heat sealed aluminum bag [108]. Therefore, it was concluded that glass vials are the most appropriate storage container for preservation of 2, 4-DNT signature odorant, as it is a storage medium that reduces contamination and the introduction of possible contaminants that could add interfering amounts of mass to the collected sample from the storage containment system.

Figure 58 shows representative chromatograms produced from storage of gauze pads in glass vials, aluminum bags, and ziploc bags in which the presence of the target odorant 2, 4-DNT from single based smokeless powder is compared with the standard (shown by the red arrow). In this figure, the effects of storage in glass vials as well as ziploc bags were the production of long-chain cycloalkanes which were present due to SPME fiber degradation after the extended extraction time $(21 \mathrm{hr})$ and from the chemical composition of the gauze pad itself. 


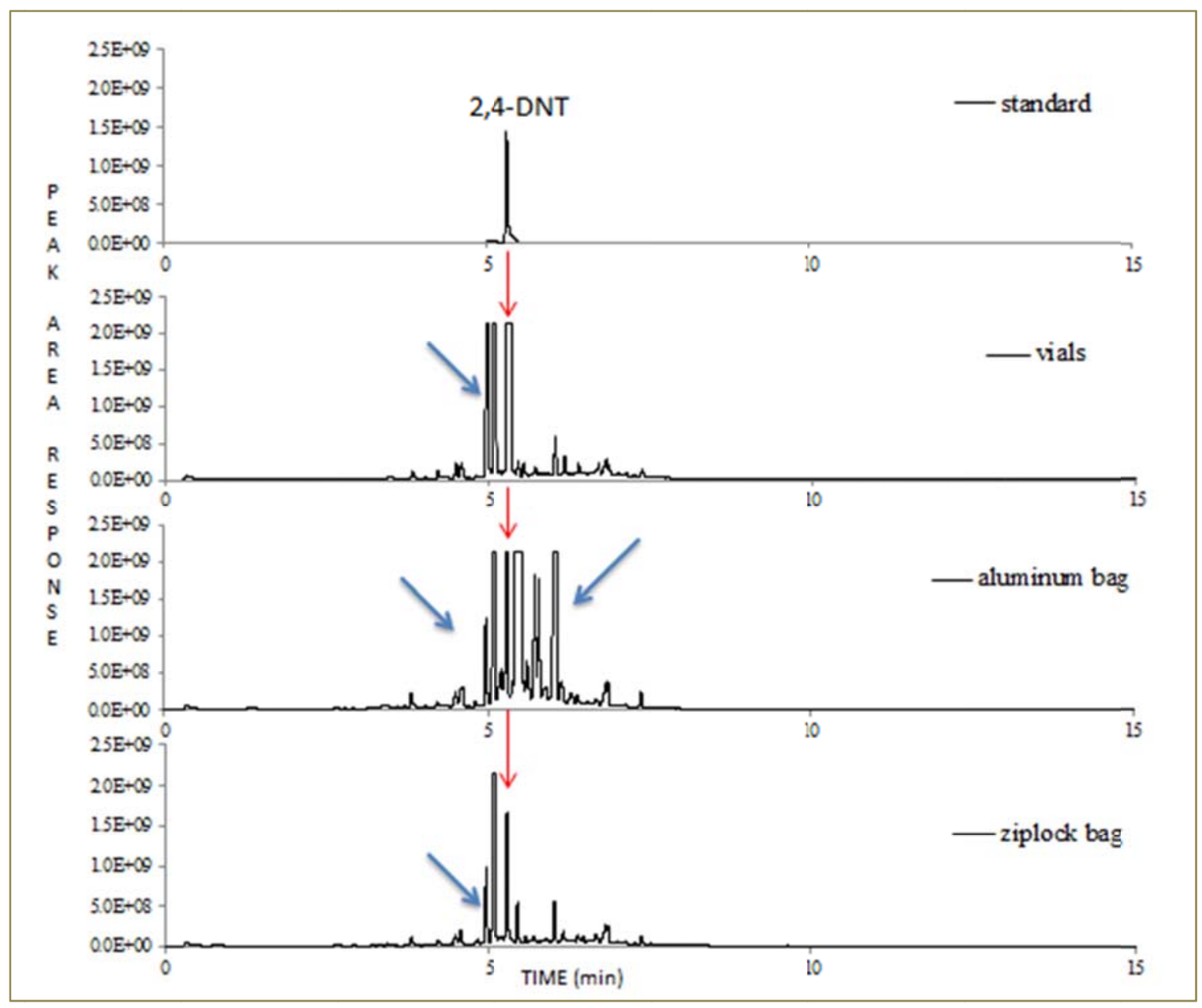

Figure 58: Comparison of Different Storage Containers after one (1) hour Storage

The effects of storage in aluminized bags can be attributed to the fact that these matrices were all heat sealed. Some of the compound classes detected during chromatographic analysis included alkanes, aldehydes, acid-methyl esters, and alcohols. The process of heat sealing may have contributed to the creation and/or release of many of these compounds which were detected on the gauze pad after storage in this container. 


\subsubsection{Conclusions}

The results obtained with the representative odorants $2 \mathrm{E} 1 \mathrm{H}$ and DMNS from $\mathrm{C} 4$ explosive material as well as NG and 2, 4-DNT from single based and double based smokeless powders respectively, demonstrate that each target volatile behaves in a distinctive manner and that the interaction of each chemical with the absorbent material (gauze pad) as well as its intrinsic physical properties such as volatility affects the amount of odor retained within the gauze matrix during storage. Other important factors include the introduction of heat which was necessary to seal the aluminum bags that contribute to the creation of many interfering compounds to be present in the headspace of the samples. In the case of $2 \mathrm{E} 1 \mathrm{H}$ and DMNB, aluminum bags not only contributed to the creation of multiple contaminants during storage but also have shown not to be able to preserve volatiles at any given time. Aluminum bags demonstrated lack of feasibility for canine detection if used on training practices.

Differences were observed in all three tested containers. Some of these differences can be attributed to important factors such: closure integrity, permeability, and wall thickness of bags. In the case of glass vials, tops were airtight and sealed with paraffin which showed to preserve volatiles in a very efficient manner. Permeability can be affected by polarity since nonpolar molecules diffuse more rapidly than polar ones. In addition, molecular size can also affect permeability because small molecules diffuse faster than larger ones. The effect of polarity and molecular size can explain for example; why NG (a larger molecule) diffused slower than 2, 4-DNT (a smaller molecule as depicted in Table 5). In the case of ziploc bags (double zipper), their wall thickness $(1 \mathrm{~mL})$ was the main reason 
for the rapid diffusion of target odorants through the pores of the packaging and its subsequent loss in the environment.

\subsection{TASK 6: FIELD EVALUATIONS OF THE HSCS USING EXPLOSIVE DETECTION CANINE TEAMS}

This task evaluates the effectiveness of the HSCS for the creation of useful training aids for canine use. Field evaluation was conducted in a blind manner at a military base with twelve (12) previously trained and certified military explosives detection canines. These canine teams were evaluated by observing alert, no alert, or interest (without final response) to the particular training aid used. Fresh and aged samples were made with the HSCS at the optimal flow rate obtained from task 1 and the optimized sampling time from task 2, and storage containment from task 4 to evaluate canine's performance and which collected aids produced the highest canine response.

\subsubsection{Field Trials}

\subsubsection{Proficiency Test}

The proficiency test was conducted in order to ensure optimal performance of the canines prior to the actual experimental design. This test was made with real explosives to familiarize canines with the same explosive materials that will be used for HSCS collection. Canine needed to achieve a passing score at a level of $90 \%$ or better to be selected to the following stage of the test. Five (5) different rooms (including a blank) were selected in which a total of twenty (20) searches were made. 
Table 37: Proficiency Test Results (n=12)

\begin{tabular}{ccccc}
\hline Explosive & $\begin{array}{c}\text { Alert Rate } \\
(\%)\end{array}$ & $\begin{array}{c}\text { Interest Rate } \\
(\%)\end{array}$ & $\begin{array}{c}\text { No Alert Rate } \\
(\%)\end{array}$ & $\begin{array}{c}\text { Combined } \\
\text { Rate of } \\
\text { Detection (\%) }\end{array}$ \\
\hline C4 & 100.0 & 0.0 & 0.0 & 100.0 \\
Det Cord & 100.0 & 0.0 & 0.0 & 100.0 \\
TNT & 83.3 & 0.0 & 16.7 & 83.3 \\
Blank & 33.3 & 0.0 & 66.7 & 33.3 \\
\hline
\end{tabular}

As expressed in

Table 37, proficiency test based on real explosive material shows that all 12 canine teams that participated alerted at a rate of $100 \%$ odor recognition to both $\mathrm{C} 4$ and detonation cord, $83.3 \%$ to TNT and $66.7 \%$ no alert rate to the blank room.

\subsubsection{HSCS Sample Collection}

\subsubsection{Aged Samples}

At the second stage of this field evaluation, canines were exposed to aged samples which were collected 3 weeks before the test. According to Table 38, detection of detonation cord provided the highest combined rate of $91.7 \%$ while only $41.7 \%$ combined alert rate for C4 and TNT was obtained. Since interest responses from canines can be subjective, two subject matter experts (SMEs) were present at the trial to determine which canine behavior could be classified as interest. In addition, the combined alert rate results from the combination of alerts and interest responses. 
Table 38: Results of Field Evaluation of 3-week Aged Samples (n=12)

\begin{tabular}{ccccc}
\hline Explosive & $\begin{array}{c}\text { Alert Rate } \\
(\%)\end{array}$ & $\begin{array}{c}\text { Interest Rate } \\
(\%)\end{array}$ & $\begin{array}{c}\text { No Alert Rate } \\
(\%)\end{array}$ & $\begin{array}{c}\text { Combined } \\
\text { Rate of } \\
\text { Detection } \\
(\%)\end{array}$ \\
\hline Control & 100.0 & 0.0 & & 100.0 \\
\hline Det Cord & 33.3 & 58.3 & 8.0 & 91.7 \\
\hline C4 & 8.3 & 33.3 & 58.3 & 41.7 \\
\hline TNT & 8.3 & 33.3 & 58.3 & 41.7 \\
\hline Blank & 8.3 & 8.3 & 83.4 & 16.7 \\
\hline
\end{tabular}

\subsubsection{Fresh samples}

The purpose of this task was to evaluate the performance of certified explosive canines to efficiently detect fresh HSCS samples collected from real explosive material at the optimal collection parameters. According to Table 39, a combined rate of higher than $63 \%$ was obtained for the selected explosives.

Table 39: Results of Field Evaluation of Fresh Samples $(n=11)$

\begin{tabular}{ccccc}
\hline Explosive & $\begin{array}{c}\text { Alert Rate } \\
(\%)\end{array}$ & $\begin{array}{c}\text { Interest Rate } \\
(\%)\end{array}$ & $\begin{array}{c}\text { No Alert Rate } \\
(\%)\end{array}$ & $\begin{array}{c}\text { Combined Rate } \\
\text { of Detection } \\
(\%)\end{array}$ \\
\hline Control & 100.0 & 0.0 & 0.0 & 100.0 \\
TNT & 72.7 & 25.0 & 9.1 & 90.9 \\
Det Cord & 72.7 & 0.0 & 27.3 & 72.7 \\
C4 & 45.5 & 40.0 & 36.4 & 63.6 \\
Blank & 54.5 & 0.0 & 45.5 & 54.5 \\
\hline
\end{tabular}




\subsubsection{Statistical analysis}

In this field evaluation, the values of the PPV percentages were calculated based on the canine's combined rate of detection. The PPV for the proficiency test was $90 \%$. The percentage calculated for fresh samples was $80.6 \%$, meaning that $80.6 \%$ of the time the canine's alert was correct while the other $19.4 \%$ were false positive. However, for aged samples PPV had a value of $91.3 \%$ which corresponds to those correct alert responses while $8.7 \%$ of the responses are considered false positive.

The NPV percentages were calculated based on the canines' combined rate of detection. For the proficiency test the NPV was $80 \%$ for those negative responses that were correct. NPV for fresh and aged samples were $38.5 \%$ and $35 \%$ respectively. Negative Predictive Values should be as close to $100 \%$ as possible indicating that the canines are not alerting falsely to items that do not pose a threat. For this field trials, the high false alert rates observed could resulted from cross contamination during HSCS sampling since substantial amounts of target odorants are found in the atmosphere when samples from real explosives are taken. To overcome this issue, testing was performed for HSCS blank samples collected in the same room where real explosives were present (with background (W/B)) and in a room free from explosive materials (without background (Wo/B)). Four (4) Local certified dogs from The Miami Dade City were

utilized for this evaluation. Samples were taken by using the HSCS device at default settings ( medium flow rate at $30 \mathrm{sec}$ ) at atmospheric conditions $\left(22.8^{\circ} \mathrm{C}\right.$ and $62.7 \%$ relative humidity) and the results obtained showed that for example if a blank sample is taken at a different place than where samples from real explosive are collected there is a probability of having a $25 \%$ alert to a clean blank while if the blank is taken in the same 
room where real explosives were sampled (at a distance of about $2 \mathrm{ft}$ ), then the possibility of having an alert due to cross contamination is double (50\%) when compared with the blank taken in a different room as seen in Table 40.

Table 40: Results of Field Evaluation of Blanks $(n=4)$

\begin{tabular}{ccccc}
\hline Sample & $\begin{array}{c}\text { Alert Rate } \\
(\%)\end{array}$ & $\begin{array}{c}\text { Interest Rate } \\
(\%)\end{array}$ & $\begin{array}{c}\text { No Alert Rate } \\
(\%)\end{array}$ & $\begin{array}{c}\text { Combined Rate } \\
\text { of Detection } \\
(\%)\end{array}$ \\
\hline $\begin{array}{c}\text { Control } \\
\text { Blank }\end{array}$ & 100.0 & 0.0 & 0.0 & 100.0 \\
$\begin{array}{c}\text { (Wo/B) } \\
\text { Blank }\end{array}$ & 0.0 & 25.0 & 75.0 & 25.0 \\
$(\mathbf{W} / \mathbf{B})$ & 25.0 & 25.0 & 50.0 & 50.0 \\
\hline
\end{tabular}

\subsubsection{Conclusions}

A PPV of $80.6 \%$ and $91.3 \%$ for fresh and aged samples respectively, refers to the canine odor recognition to target volatiles dynamically collected with the HSCS. This clearly demonstrates the effectiveness of the HSCS in collecting volatiles for the creation of canine training aids that can be used immediately or up to several weeks (3) by following optimal established parameters and proper storage conditions. The lower PPV value obtained for fresh samples was as a result of the higher rate of false positives canines displayed to HSCS blank samples. The best method for collection of HSCS blank samples in the presence of explosive material without causing cross-contamination still reminds to be determined. 


\section{GENERAL CONCLUSIONS}

It is the purpose of this research to describe a novel method for the in situ collection of volatiles released from real explosive material by the use of an air flow device called the human scent collection system (HSCS). The volatile profile obtained from an odor source where volatiles are constantly emitted strongly depends on whether the headspace volatiles are contained (concentrated) or actively relayed to the collection device in this case via HSCS air flow. This open sampling scheme is simple to carry out but results in variable losses of target volatiles and contamination by background odors. In this research, in order to partially isolate the odor source crystal jars $(8 \mathrm{oz})$ were utilized to concentrate volatile emissions and to prevent direct contact and contamination to the odor source. According to the results obtained, the HSCS device has proven to be effective in the collection of target volatiles onto an adsorbent material that were instrumentally detected at the nanogram level. In addition, optimal parameters such flow rate and sampling time were developed for the collection of signature volatiles from different explosive materials.

Different Collection techniques were utilized: dynamic and static. In dynamic flow, the active air flow of the HSCS moves headspace volatiles through an adsorbent material. In the static flow system there is no air flow present, therefore adsorption of volatiles relies primary on the volatilization of the odorants themselves. The main benefit of dynamic collection is that collection of volatiles is easier and faster (30 and 60 seconds) while static technique does not require the use of the equipment, collects odorants at greater amounts in which longer exposure times are required for optimal collection. In addition, 
glass vials were selected as the optimal storage containment since they have shown to preserve volatiles for longer periods with minimal levels of contamination.

Trial results had shown the effectiveness of the HSCS system since canines were able to detect explosive odorants dynamically collected. The HSCS system described here can then be used to collect explosive odorants from a wide range of odor sources. If the HSCS device is used accordingly with the recommendations given, it will warranty the successful creation of useful and non-hazardous training aids which can be used immediately or up to several weeks (3) after collection under proper storage conditions. The new training aids can replace the use and transport of real explosive material which represents a high risk not only to the canine but also to the handler.

\section{RECOMMENDATIONS}

In view of its capability for collecting trace amounts of explosive evidence, the HSCS device has proven its effectiveness for the dynamic collection of explosive volatiles. According to the results obtained during this project, optimal parameters for HSCS collection of each of the representative explosive volatiles from the four explosive families are as expressed in Table 41. Therefore, if the recommendations given in this table are followed, canine explosive detection will highly benefit through the use of scientifically validated training aids. These reliable non-hazardous training aids will not diminish canine's ability to detect explosives. On the contrary, they will provide optimal canine training scenarios where real explosive material is no longer necessary. In addition, the use of the HSCS for the creation of training aids will also provide a novel 
approach for collection of explosive samples which are emerging from current explosive terrorist attacks worldwide.

Table 41: Optimal Parameters for HSCS Collection of Explosive Volatiles

\begin{tabular}{|c|c|c|c|}
\hline $\begin{array}{l}\text { Explosive } \\
\text { Family }\end{array}$ & Explosive & $\begin{array}{c}\text { HSCS } \\
\text { Collection } \\
\text { Time (seconds) } \\
\end{array}$ & $\begin{array}{c}\text { HSCS } \\
\text { Flow Rate }\end{array}$ \\
\hline Nitro-alkanes & $\mathrm{C} 4$ & 60 & High \\
\hline Nitro-amines & $\mathrm{C} 4$ & 60 & High \\
\hline Nitrate-esters & $\begin{array}{c}\text { Det. Cord and Double } \\
\text { Based Smokeless } \\
\text { Powder }\end{array}$ & 30 & High \\
\hline $\begin{array}{c}\text { Nitro- } \\
\text { aromatics }\end{array}$ & $\begin{array}{c}\text { TNT } \\
\text { Single Based Smokeless } \\
\text { Powder }\end{array}$ & $\begin{array}{l}60 \\
30\end{array}$ & $\begin{array}{l}\text { Low } \\
\text { High }\end{array}$ \\
\hline
\end{tabular}




\section{REFERENCES}

1. Furton, K.G., Myers, L.J. The Scientific Foundations and Efficacy of the Use of Canines as Chemical Detectors for Explosives. Talanta. 2001; 54(3): 487-500.

2. Harper, R.J., Almirall, J.R., Furton, K.G. Identification of Dominant Odor Chemicals Emanating from Explosives for Use in Developing Optimal Training Aid Combinations and Mimics for Canine Detection. Talanta. 2005; 313-317.

3. Harper, R.J. Improving the Scientific Reliability of Biological Detection of Explosives by Canis Familiaris: Active Odor Signatures and Their Implications. Florida International University, 2005.

4. Lai, H., Leung. A., Magee, M., Almirall, J. R., Identification of Volatile Chemical Signatures from Plastic Explosives by SPME-GC/MS and Detection by Ion Mobility Spectrometry, Analytical and Bioanalytical Chemistry. 2010; 396: 2997-3007.

5. Akhavan, J. The Chemistry of Explosives3th Ed. Cambridge: The Royal Society of Chemistry, 2011.

6. Marshal, M., Oxley. J., Aspects of Explosive Detection, 1st Ed. Oxford, UK: Elsevier, 2011.

7. Agrawal, J. P. High energy materials: propellants, explosives and pyrotechnics. John Wiley \& Sons, 2010.

8. Makinen, M., Nousiainen, M., Sillanpa, M., Ion Spectrometric Detection Technologies for Ultra-Traces of Explosives: A Review, Journal of Mass Spectrometry. 2011; 30: 940973.

9. Beveridge, A., Forensic Investigation of Explosives, 2nd Ed. Florida, USA: CRC Press, 2012. 
10. Caygill, J., Davis. S., Higson, F., Seamus. P.J., Current Trends in Explosive detection Techniques, Talanta 2012; 88: 14-29.

11. Perr, J.M., Furton, K.G., Almirall, J.R., Solid Phase Microextraction Ion Mobility Spectrometer Interface for Explosive and Taggant Detection, J. Sep. Sci. 2005; 28: 177183.

12. Gottfried, J.L., De Lucia, F.C. Jr., Munson, C.A., Miziolek, A.W., Laser-Induced Breakdown Spectroscopy for Detection of Explosives Residues: A Review of Recent Advances, Challenges, and Future Prospects. Analytical and Bioanalytical Chemistry. 2009; 395: 283-300.

13. Nilles, J. M., Connell, T. R., Stokes, S. T., Dupont Durst, H. Explosives detection using direct analysis in real time (DART) mass spectrometry. Propellants, Explosives, Pyrotechnics. 2010; 35(5): 446-451.

14. Justes, D. R., Talaty, N., Cotte-Rodriguez, I., Cooks, R. G. Detection of explosives on skin using ambient ionization mass spectrometry. Chem. Commun. 2007 ; (21), 21422144.

15. Sanders, N.L., Kothar, S., Huang, G., Salazar, G., Cooks, R.G., Detection of Explosives as Negative Ions Directly from Surfaces using a Miniature Mass Spectrometer, Analytical Chemistry. 2010; 82: 5313-5316.

16. Engel, Y., Elnathan, R., Pevzner, A., Davidi, G., Flaxer, E., Patolsky, F., Supersensitive Detection of Explosives by Silicon Nanowire Arrays, Angew. Chem. Int. Ed. 2010; 49: 6830-6835.

17. Röck, F., Barsan, N., Weimar, U. Electronic nose: current status and future trends. Chemical reviews. 2008; 108(2): 705-725.

18. Wang, J., Electrochemical Sensing of Explosives, Elec. 2007; 19: 415-423. 
19. Frederickx, C., Verheggen, F., Haubruge, E. Biosensors in forensic sciences. Biotechnology, Agronomy, and Society Environment. 2011; 15(4): 449-458.

20. Leitch, O., Anderson, A., Paul Kirkbride, K., Lennard, C. Biological organisms as volatile compound detectors: A review. Forensic Science International. 2013; 232(1): 92103.

21. Yinon, J., Zitrin, S. Modern methods and applications in analysis of explosives. John Wiley \& Sons, 1996.

22. Poling, A., Weetjens, B.J., Cox, C., Beyene, N.W., Sully, A. Using giant African pouched rats (Cricetomys gambianus) to detect landmines, Psych. Rec. 2010; 60 (4) : $715-728$.

23. Rains, G.C., Tomberlin, J.K., Kulasiri, D. Using insect sniffing devices for detection. Trends Biotechnology. 2008; 26(6): 288-294.

24. Bromenshenk, J.J, Henderson, C.B., Smith, G.C. Appendix S. Biological systems, Alternatives for Landmine Detection, Montana, 2003; 273 -283.

25. SPAWAR, 1992. U.S. Navy Marine Mammal Program.

http://www.public.navy.mil/spawar/Pacific/71500/Pages/default.aspx Accessed 12-2014.

26. Lazarowski, L., Dorman, D. C. Explosives detection by military working dogs: Olfactory generalization from components to mixtures. Applied Animal Behaviour Science. 2014; 151: 84-93.

27. Sandercock, P. M. L. Fire investigation and ignitable liquid residue analysis-A review: 2001-2007. Forensic science international. 2008; 176(2): 93-110.

28. Furton, K.G.; Hsu, Y.; Luo, T.; Wang, J.; Rose, S. Odor signatures of cocaine analyzed by GC/MS and threshold levels of detection for drug detection canines. Curr. Top. Forensic Sci., Proc. Meet. Int. Assoc. Forensic Sci.1997; 14(2): 329-32. 
29. Lai, H., Corbin, I., Almirall, J. R. Headspace sampling and detection of cocaine, MDMA, and marijuana via volatile markers in the presence of potential interferences by solid phase microextraction-ion mobility spectrometry (SPME-IMS). Analytical and Bioanalytical Chemistry. 2008; 392(1-2): 105-113.

30. Rebmann, A., David E., Sorg, M.H. Cadaver dog handbook: forensic training and tactics for the recovery of human remains. Boca Raton, FL, USA: CRC Press, 2000.

31. Curran, A. M., Prada, P. A., Furton, K. G. Canine human scent identifications with post-blast debris collected from improvised explosive devices. Forensic Science International. 2010; 199(1): 103-108.

32. Lasseter, A. E., Jacobi, K. P., Farley, R., Hensel, L. Cadaver dog and handler team capabilities in the recovery of buried human remains in the Southeastern United States. Journal of Forensic Sciences. 2003; 48(3): 617-621.

33. Harper, R.J.; Curran, A.M.; Almirall, J.R.; Furton, K.G. Developing the Science behind Canine Olfaction of Forensic Specimens. Proc. of the 1st OBSSA, 2004.

34. http://www.allstates-k9.com/upload/dogs $\% 20$ detecting $\% 20$ cell $\% 20$ phones.pdf Accessed 12-2014.

35. Furton, K.G.; Harper, R. Detection of Ignitable Liquid Residues in Fire Scenes Accelerant Detection Canine (ADC) Teams and other Field Tests, in Advances in Forensic Science Techniques: Interpretation of Fire Scene Evidence, CRC Press, Boca Raton, FL.2004.

36. $\mathrm{Vu}, \mathrm{D} . \mathrm{T}$. Characterization and aging study of currency ink and currency canine training aids using headspace SPME/GC-MS. Journal of Forensic Sciences. 2003; 48(4):754-770.

37. Kauhanen, E.; Harri, M.; Nevalainen, A.; Nevalainen, T. Validity of detection of microbial growth in buildings by trained dogs. Environment International. 2002; 28(3): 153-157. 
38. Griffith, R.T.; Jayachandran, K.; Whitstine, W.; Furton, K.G. Differentiation of Toxic Molds via Headspace SPME-GC/MS and Canine Detection Sensors. 2007; 7: 1415-1427.

39. Pickel, D., Manucy, G. P., Walker, D. B., Hall, S. B., Walker, J. C. Evidence for canine olfactory detection of melanoma. Applied Animal Behaviour Science. 2004; 89(1): 107-116.

40. Harper, R.J., Furton, K.G., Biological detection of explosives. In: Yinon J., ed. Counterterrorist detection techniques of explosives. Amsterdam, The Netherlands; Oxford, UK: Elsevier. 2007: 395-431.

41. Quignon, P., Rimbault, M., Robin, S., Galibert, F. Genetics of canine olfaction and receptor diversity. Mammalian Genome. 2012; 23(1-2): 132-143.

42. Craven, B.A., Neuberger, T., Paterson, E.G.,Webb A.G., Josephson, E.M., Morrison, E.E., Settles, G.S. Reconstruction and Morphometric analysis of the nasal airway of the dog (Canis Familiaris) and implications regarding olfactory airflow. The Anatomical Record. 2007; 290: 1325-1340.

43. http://www.aces.edu/pubs/docs/U/UNP-0066/UNP-0066.pdf Accessed 12-2014.

44. http://www.readingdogwalker.com/2013/08/a-dogs-sense-of-smell.html 12-2014.

Accessed

45. Sjaastad, Ø.V., Sand, O., Hove K. Physiology of Domestic Animals. 2nd Ed. Oslo: Scandinavian Veterinary Press, 2010.

46. Wells, D. L., Hepper, P. G. Directional tracking in the domestic dog (Canis familiaris). Applied Animal Behaviour Science. 2003; 84(4): 297-305.

47. http://www.detectiondogs.com/articles/ScentGeneralization.pdf Accessed12-2014 
48. Kaldenbach, J. K9 scent detection: My favorite judge lives in a kennel. Detselig Enterprises Ltd, Alberta, Canada, 1998.

49. Scientific Working Group on Dog \& Orthogonal detector Guidelines. Approved guidelines. http://www.swgdog.org/ Accessed on April 2012.

50. Kvam, A. L. The Canine Kingdom of Scent: Fun Activities Using Your Dog's Natural Instincts. Dogwise Publishing, 2011.

51. Law Enforcement. Legal Rev., 23 (1994) 6.

52. Lorenzo, N., Wan T., Harper, R.J., Hsu Y.L., Chow, M., Rose S., Furton, K.G. Laboratory and Field Experiments Used to Identify Canis Familiaris Active Odor Signature Chemicals from Drugs, Explosives and Humans. Analytical and. Bioanalytical Chemistry. 2003; 376: 1212-1224.

53. Harper, R.J., Almirall, J.R., Furton, K.G., Improving the Scientific Reliability of BioLogical Detection of Explosives by Canis Familiaris through Active Odor Signatures and Their Implications, in: Proceedings of the 8th International Symposium on the Analysis and Detection of Explosives (ISADE), Ottawa, Canada, 2004.

54. Joshi, M., Delgado. Y., Guerra, P., Lai. H., Almirall, J.R., Detection of Odor Signatures of Smokeless Powders Using Solid Phase Microextraction Coupled To an Ion Mobility Spectrometer, For. Sci. Inter. 2009; 188: 112-118.

55. Johnston, J.M. Enhanced canine explosives detection. Institute for Biological Detection Systems, Auburn University, Alabama, 1998.

56. Ensminger, J.J., Police and Military Dogs: Criminal Detection, Forensic Evidence, and Judicial Admissibility, 1st Ed. New York, USA: CRC Press, 2011: 221-234.

57. Furton, K.G., Harper, R. J., Florida USA, 2008; Patent \# 20080295783. 
58. Macias, M.S., Harper, R.J., Furton, K.G. A comparison of real versus simulated contraband VOCs for reliable detector dog training utilizing SPME-GC-MS. American Biotechnology Laboratory. 2008; 26(6):26-7.

59. Beltz, K. The Development of Calibrants through Characterization of Volatile Organic Compounds from Peroxide Based Explosives and a Non-target Chemical Calibration Compound. Dissertation submitted for Doctor of Philosophy in Chemistry, Florida International University, 2013.

60. Oxley, J.C., Smith, J.L., Moran, J., Nelson, K., Utley, W.E. Training dogs to detect Triacetone Triperoxide (TATP). Proc.SPIE-Int.Soc.Opt.Eng. 5403[Pt. 1, Sensors, and Command, Control Communications, and Intelligence (C31) Technologies for Homeland Security and Homeland Defense III], 349-353. 2004.

61. Schoon, A., Gotz, S., Heuven, M., Vogel, M., Karst, U. Training and Testing Explosive Detection Dogs in Detecting Triacetone Triperoxide. Forensic Science Communications. 2006; 8(4).

62. Frye v. United States, 293 Fed. 1013 (D.C. Cir. 1923).

63. Daubert v. Merrell Dow Pharmaceuticals, Inc. (1993) 509 US 579.

64. Kumho Tire, Inc. v. Carmichael, 119 S.Ct.1167 (1999).

65. Hodge v. State, 98 Ala. 10 (Ala. 1893).

66. State v. Hall, 4 Ohio Dec.147, 148 (Ohio Misc. 1896).

67. Zanoni, M. M., Morris, A., Messer, M., Martinez, R. Forensic evidence canines: status, training and utilization. In American Academy of Forensic Sciences Annual Meeting. 1998, from http://www.csst.org/forensic evidence canines.html. Accessed 122014. 
68. Walker, J.S. Using Drug-Detection Dogs. FBI Law Enforcement Bulletin. 2001

Retrieved from http://leb.fbi.gov/2001-pdfs/leb-april-2001 Accessed 12-2014.

69. Illinois v. Roy I. Caballe., No. 03-923 (January 24, 2005).

70. Arizona v. Quastling, 536P.2d 226 (Az. App. 1975).

71. http://www.academia.edu/4207329/The_Nose_Knows_A_Case_Analysis_of_Scent Dog_Evidence Accessed 12-2014.

72. https://www.atf.gov/content/Explosives/explosives-enforcement/accelerant-andexplosives-detection-canines Accessed 12-2014.

73. North American Police Work Dog Association Certification Rules (N.A.P.W.D.A.) (1998). Perry OH: N.A.P.W.D.A., 1-23.

74. IFRI/NFSTC Detector Dog Certification Guidelines 2005 Accessed 12-2014. from http://www2.fiu.edu/ ifri/detector_dogs.htm

75. Furton, K.G.; Heller, D.P. Advances in the reliable location of forensic specimens through research and consensus best practice guidelines for $\operatorname{dog}$ and orthogonal instrumental detectors. Canadian Journal of Police \& Security Services. 2005; 3(2): 97107.

76. Lit, L., Schweitzer, J. B., Oberbauer, A. M. Handler beliefs affect scent detection dog outcomes. Animal Cognition.2011; 14(3): 387-394.

77. Florida v. Jardines, 569 U.S.(2013).

78. Vas, G., Karoly V. Solid-phase microextraction: a powerful sample preparation tool prior to mass spectrometric analysis. Journal of Mass Spectrometry. 2004; 39(3): 233254. 
79. Wercinski, S.A. Solid Phase Microextraction A Practical Guide. New York, Marcel Dekker, Inc. 1999.

80. Zhang, Z., Yang, M.J., Pawliszyn, J. Solid-Phase Microextraction. A Solvent-Free Alternative for Sample Preparation. Analytical Chemistry. 1994; 66(17): 844A-852A.

81. Pawliszyn, J. Theory of solid-phase microextraction. Journal of Chromatographic Science. 2000; 38(7):270-278.

82. Solid Phase Microextraction: Theory and Optimization of Conditions. Supelco, Bellefonte, Pa. Bulletin 923.

https://www.sigmaaldrich.com/Graphics/Supelco/objects/4600/4547.pdf. Accessed 122014.

83. Ouyang, G., Pawliszyn, J. SPME in environmental analysis. Analytical and Bioanalytical Chemistry. 2006; 386(4): 1059-1073.

84. Aulakh, J. S., Malik, A. K., Kaur, V., Schmitt-Kopplin, P. A Review on solid phase micro extraction-high performance liquid chromatography (SPME-HPLC) analysis of pesticides. Critical reviews in analytical chemistry. 2005; 35(1): 71-85.

85. Mughari, A. R., Vázquez, P. P., Galera, M. M. Analysis of phenylurea and propanil herbicides by solid-phase microextraction and liquid chromatography combined with post-column photochemically induced fluorimetry derivatization and fluorescence detection. Analytica Chimica Acta. 2007; 593(2): 157-163.

86. King, A. J., Readman, J. W., Zhou, J. L. The application of solid-phase microextraction (SPME) to the analysis of polycyclic aromatic hydrocarbons (PAHs). Environmental Geochemistry and Health. 2003; 25(1): 69-75.

87. Kataoka, H., Lord, H.L, Pawliszyn, J. Applications of solid-phase microextraction in food analysis. Journal of Chromatography Analysis. 2000; 880(1): 35-62. 
88. Yang, X., Peppard, T. Solid-phase microextraction for flavor analysis. Journal of Agricultural and Food Chemistry. 1994; 42(9): 1925-1930.

89. Tunick, M. H., Iandola, S. K., Van Hekken, D. L. Comparison of SPME Methods for Determining Volatile Compounds in Milk, Cheese, and Whey Powder. Foods. 2013; 2(4): 534-543.

90. Wardencki, W., Sowiński, P., Curyło, J. Evaluation of headspace solid-phase microextraction for the analysis of volatile carbonyl compounds in spirits and alcoholic beverages. Journal of Chromatography A. 2003; 984(1): 89-96.

91. Li, Jia, Qiong Su, Ke-Yao Li, Chu-Feng Sun, and Wen-Bo Zhang. Rapid analysis of phthalates in beverage and alcoholic samples by multi-walled carbon nanotubes/silica reinforced hollow fibre-solid phase microextraction. Journal of Agricultural and Food Chemistry. 2013; 141(4): 3714-3720.

92. De León-Rodríguez, A., González-Hernández, L., Barba de la Rosa, A. P., EscalanteMinakata, P., López, M. G. Characterization of volatile compounds of mezcal, an ethnic alcoholic beverage obtained from Agave salmiana. Journal of Agricultural and Food Chemistry. 2006; 54(4): 1337-1341.

93. Strano-Rossi, S., Chiarotti, M. Solid-phase microextraction for cannabinoids analysis in hair and its possible application to other drugs. Journal of Analytical Toxicology. 1999; 23(1): 7-10.

94. Kumar, A., Malik, A. K., Matysik, F. M. Analysis of biological samples using solidphase microextraction. Bioanalytical Reviews. 2009; 1(1): 35-55.

95. Pragst, F. Application of solid-phase microextraction in analytical toxicology. Analytical and Bioanalytical Chemistry. 2007; 388(7): 1393-1414.

96. Almirall, J. R., Wang, J., Lothridge, K., Furton, K. G. The detection and analysis of ignitable liquid residues extracted from human skin using SPME/GC. Journal of Forensic Sciences. 2000; 45(2): 453-461. 
97. Furton, K. G., Wang, J., Hsu, Y. L., Walton, J., Almirall, J. R. The use of solid-phase microextraction - gas chromatography in forensic analysis. Journal of Chromatographic Science. 2000; 38(7): 297-306.

98. Furton, K. G., Wu, L., Almirall, J. R. Optimization of solid-phase microextraction (SPME) for the recovery of explosives from aqueous and post-explosion debris followed by gas and liquid chromatographic analysis. Journal of Forensic Sciences. 2000; 45(4): 857-864.

99. Skoog, D.A., Holler J.F., Nieman, T.A. Principles of Instrumental Analysis. 7th Ed. Ontario, Thomson Learning Inc. 2007.

100. Bouchonnet, S. Introduction to GC-MS Coupling. CRC Press, Boca Raton, 2013.

101. Miller, J.N, Miller, JC. Statistics and Chemometrics for Analytical Chemistry. 6th Ed.UK, Pearson Prentice Hall, 2005.

102. Wonnacott, T. H., Wonnacott, R. J. Introductory statistics. 5th Ed. New York, Jhon Wiley \& Sons, 1972.

103. Curran, A. M., Prada, P.A., Furton, K. G. The differentiation of the volatile organic signatures of individuals through SPME-GC/MS of characteristic human scent compounds, Journal. of Forensic Science. 2010; 55(1): 50-57.

104. http://www.omega.com/green/pdf/airflow meas ref.pdf Accessed July 2012.

105. Lawrence, M. G. The relationship between relative humidity and the dewpoint temperature in moist air: a simple conversion and applications. Bulletin of the American Meteorological Society 2005, 86 (2).

106. Huang, H.; Haghighat, F.; Blondeau, P. Volatile organic compound (VOC) adsorption on material: influence of gas phase concentration, relative humidity and VOC type. Indoor Air. 2006; 16 (3): 236-247. 
107 . DeGreeff, L. E., Furton, K. G. Collection and identification of human remains volatiles by non-contact, dynamic airflow sampling and SPME-GC/MS using various sorbent materials. Analytical and bioanalytical chemistry. 2011; 401(4): 1295-1307.

108. Hudson, D. T.; Curran, A. M.; Furton, K. G. The Stability of Collected Human Scent Under Various Environmental Conditions. Journal of Forensic Sciences 2009, 54 (6), 1270-1277. 


\section{APPENDIX}

\section{A. Calibration Curves of Selected Compounds}

The initial evaluation of selected representative explosive odors has been used for optimization purposes of the HSCS. Prior to direct sampling of real explosive material with the airflow dynamic system, proper calibration procedures were conducted to verify the optimal detection of each analyte with the analytical instrument. The experimental design included an in-depth evaluation of four of the representative odors: 2-ethyl-1hexanol (2E1H), 2, 3 -Dimethyl- 2, 3-Dinitrobutane (DMNB), 2,4-dinitrotoluene (2, 4DNT), and nitroglycerin (NG). Therefore, a calibration curve was prepared for each of the four representative VOCs by dilution in acetonitrile or methylene chloride solvent different concentrations. Prior to injection, standards were prepared as follows:

- $\quad 31$ ul of 2-ethyl-1-hexanol standard (Sigma \& Aldrich $\geq 99.6 \%$ ) were diluted in $25 \mathrm{ml}$ flask with methylene chloride. Eventually, dilutions of $0.5,1 \mathrm{ppm}, 5 \mathrm{ppm}, 10 \mathrm{ppm}$, 20 ppm, 40 ppm, 60 ppm, 80 ppm and 100 ppm were prepared in $5 \mathrm{ml}$ flask and placed in $2 \mathrm{ml}$ vials for GC/MS analysis.

- $\quad 0.0259 \mathrm{~g}$ of DMNB standard (Sigma \& Aldrich $98 \%$ ) was diluted in $25 \mathrm{ml}$ flask with methylene chloride. Subsequently, dilutions of $0.5,1 \mathrm{ppm}, 5 \mathrm{ppm}, 10 \mathrm{ppm}, 20 \mathrm{ppm}$, $40 \mathrm{ppm}, 60 \mathrm{ppm}, 80 \mathrm{ppm}$ and $100 \mathrm{ppm}$ were prepared in $5 \mathrm{ml}$ flask and placed in $2 \mathrm{ml}$ vials for GC/MS analysis. 
- $\quad 100 \mathrm{ul}$ of diluted 2,4 DNT standard (Sigma\& Aldrich $1000 \mathrm{ug} / \mathrm{ml}$ in EtOH-MeOH ) was mixed with $900 \mathrm{ul}$ of acetonitrile. Eventually, dilutions of $0.05 \mathrm{ppm} 0.1 \mathrm{ppm}, 0.3$ ppm, $0.5 \mathrm{ppm}, 1 \mathrm{ppm}, 2 \mathrm{ppm}$, and $3 \mathrm{ppm}$ were prepared in $5 \mathrm{ml}$ flask and placed in $2 \mathrm{ml}$ vials for GC/ECD analysis.

- $\quad 100 \mathrm{ul}$ of diluted Nitroglycerin standard (Sigma\& Aldrich $1000 \mathrm{ug} / \mathrm{ml}$ in EtOH$\mathrm{MeOH}$ ) was mixed with $900 \mathrm{ul}$ of acetonitrile. Eventually, dilutions of $0.05 \mathrm{ppm} 0.1$ ppm, $0.3 \mathrm{ppm}, 0.5 \mathrm{ppm}, 1 \mathrm{ppm}, 2 \mathrm{ppm}$, and $3 \mathrm{ppm}$ were prepared in $5 \mathrm{ml}$ flask and placed in $2 \mathrm{ml}$ vials for $\mathrm{GC} / \mathrm{ECD}$ analysis.

Chromatograms for four representative compounds: 2E1H, DMNB, 2,4 DNT, and NG along with calibration curves for each are depicted in the Figure below. All of the four components studied had a linear fit with correlation coefficients $>0.99$ as seen in the following Table:

Table 42: Compound List, Including Retention Times, Masses, and a Summary of Results for Precision and Linearity

\begin{tabular}{|c|c|c|c|c|}
\hline $\begin{array}{l}\text { RETENTION } \\
\text { TIME (min) }\end{array}$ & $\begin{array}{l}\text { COMPOUND } \\
\text { NAME }\end{array}$ & $\begin{array}{l}\text { MOLECULAR } \\
\text { MASS (g/mol) }\end{array}$ & $\mathbf{R}^{2}$ & $\begin{array}{l}\text { CALIBRATION } \\
\text { RANGE (ug/ml) }\end{array}$ \\
\hline 10.5 & $\begin{array}{c}\text { 2-Ethyl-1-Hexanol } \\
(2 \mathrm{E} 1 \mathrm{H})\end{array}$ & 130 & 0.9979 & $0.5-100$ \\
\hline 13.3 & $\begin{array}{l}\text { 2, } 3 \text {-Dimethyl- 2, } \\
\text { 3-Dinitrobutane } \\
\text { (DMNB) }\end{array}$ & 176 & 0.9986 & $0.5-100$ \\
\hline 5.4 & $\begin{array}{l}\text { 2,4-Dinitrotoluene } \\
\text { (2,4 DNT) }\end{array}$ & 182 & 0.9952 & $0.05-3$ \\
\hline 8.3 & $\begin{array}{c}\text { Nitroglycerin } \\
\text { (NG) }\end{array}$ & 227 & 0.9941 & $0.05-3$ \\
\hline
\end{tabular}



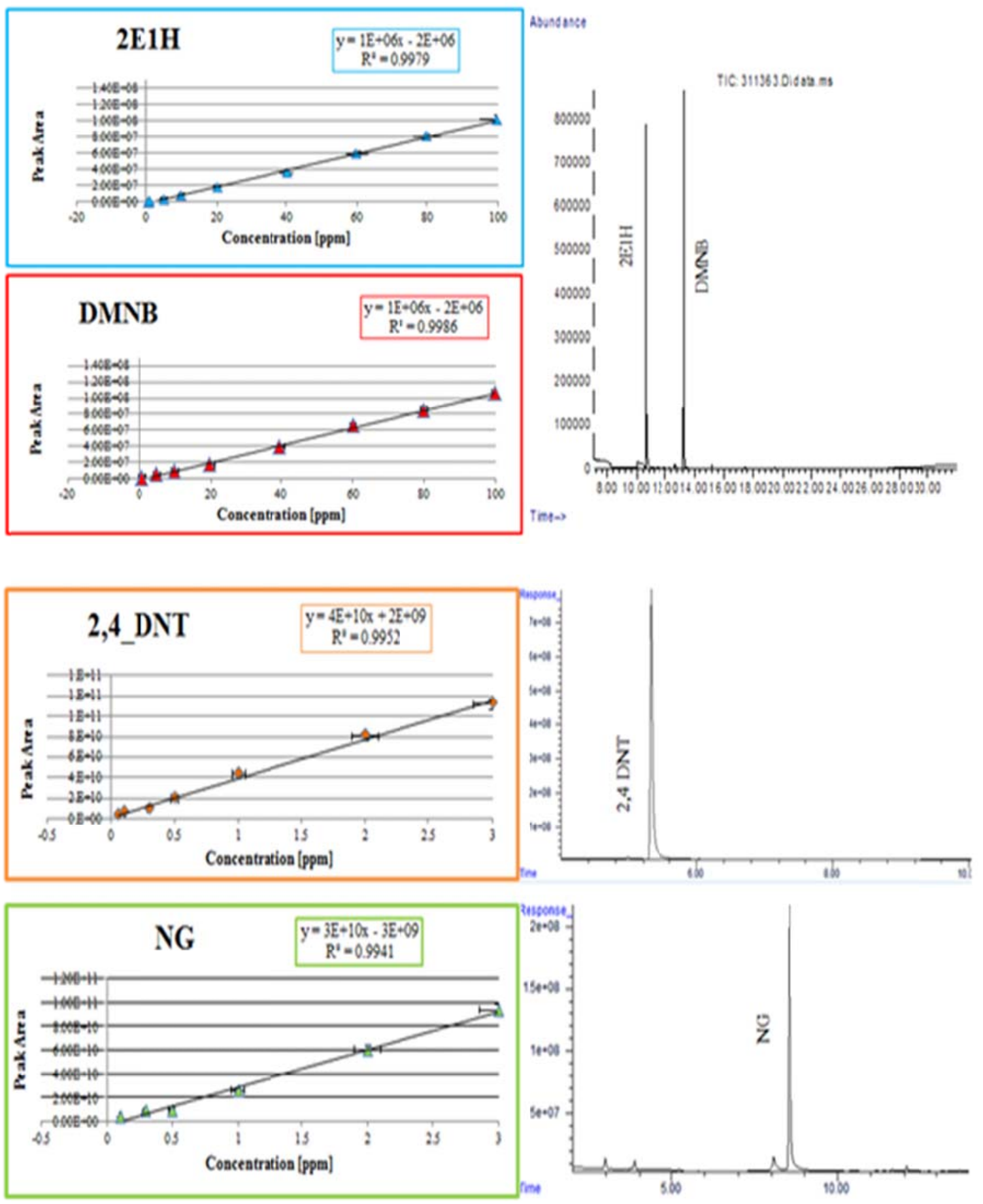

Figure 59: Calibration Curves for 2E1H, DMNB, 2,4-DNT, NG, and the Extracted Ion Chromatograms for Selected Compounds 